Published in final edited form as:

Compr Physiol. 2012 July ; 2(3): . doi:10.1002/cphy.c110051.

\title{
Gap Junctions
}

\author{
Morten Schak Nielsen ${ }^{1}$, Lene Nygaard Axelsen ${ }^{1}$, Paul L. Sorgen ${ }^{2}$, Vandana Verma ${ }^{3}$, Mario \\ Delmar ${ }^{4}$, and Niels-Henrik Holstein-Rathlou ${ }^{*}, 1$ \\ ${ }^{1}$ Department of Biomedical Sciences and The Danish National Research Foundation Centre for \\ Cardiac Arrhythmia, Faculty of Health Sciences, University of Copenhagen, Copenhagen, \\ Denmark
}

${ }^{2}$ Department of Biochemistry and Molecular Biology, University of Nebraska Medical Center, Omaha, Nebraska

${ }^{3}$ Center for Arrhythmia Research, Department of Internal Medicine, University of Michigan, Ann Arbor, Michigan

${ }^{4}$ The Leon H. Charney Division of Cardiology, New York University School of Medicine, New York, New York

\section{Abstract}

Gap junctions are essential to the function of multicellular animals, which require a high degree of coordination between cells. In vertebrates, gap junctions comprise connexins and currently 21 connexins are known in humans. The functions of gap junctions are highly diverse and include exchange of metabolites and electrical signals between cells, as well as functions, which are apparently unrelated to intercellular communication. Given the diversity of gap junction physiology, regulation of gap junction activity is complex. The structure of the various connexins is known to some extent; and structural rearrangements and intramolecular interactions are important for regulation of channel function. Intercellular coupling is further regulated by the number and activity of channels present in gap junctional plaques. The number of connexins in cell-cell channels is regulated by controlling transcription, translation, trafficking, and degradation; and all of these processes are under strict control. Once in the membrane, channel activity is determined by the conductive properties of the connexin involved, which can be regulated by voltage and chemical gating, as well as a large number of posttranslational modifications. The aim of the present article is to review our current knowledge on the structure, regulation, function, and pharmacology of gap junctions. This will be supported by examples of how different connexins and their regulation act in concert to achieve appropriate physiological control, and how disturbances of connexin function can lead to disease. (C) 2012 American Physiological Society. Compr Physiol 2:1981-2035, 2012.

\section{Introduction}

Gap junctions are integral membrane proteins that enable the direct cytoplasmic exchange of ions and lowmolecular weight metabolites between adjacent cells. These connections are essential in many aspects of animal physiology including propagation of electrical signals and coordination of cell signaling by transfer of second messengers. The proteins that form gap junctions differ between vertebrates and nonvertebrates. In vertebrates, gap junctions are formed by connexins, whereas the gap junction proteins of nonvertebrate animals are termed

Copyright (C) American Physiological Society

*Correspondence tonhhr@sund.ku.dk; schack@sund.ku.dk. 
innexins (517). Connexins share structural features with innexins though there is no amino acid sequence homology between the two. Proteins with amino acid sequence homology to innexins have been identified in vertebrates (500). These proteins, called pannexins, are able to form intercellular channels when overexpressed, but studies show that native pannexins are likely glycosylated in their extracellular domains and do not form cell-cell channels (507). Therefore, it is suggested that they act as hemichannels (114).

The aim of this article is solely to review our current knowledge of connexins. A simple search on PubMed on "gap junction OR connexin" yields more than 15,000 hits. This shows that a huge amount of scientific work has been conducted in this field, and obviously it is impossible to cover every detail in this article. The article aims to cover the basic principles of connexin structure, how connexin expression, transcription, turnover, and trafficking are regulated, as well as how mature gap junction channels are affected by gating and posttranslational modification. Finally, some insight to the physiological function of gap junctions and their involvement in various diseases is given, along with a status of experimental pharmacological treatments targeting gap junctions.

\section{Basic Properties of Connexins}

\section{Connexin structure}

Gap junctions are formed by the apposition of connexons from adjacent cells, where each connexon is formed by six connexin proteins (Fig. 1A). There are 21 different connexin genes in the human genome and 20 in mice. Connexins are tetraspan transmembrane (TM) domain proteins with intracellular N- and C-termini (Fig. 1B). Two extracellular loops (E1 and E2) provide continuity between TM domains TM1-TM2 and TM3-TM4, while the region between TM2 and TM3 (the "cytoplasmic loop;" CL) is located in the intracellular space. Connexins are expressed in all tissues except differentiated skeletal muscle, erythrocytes, and mature sperm cells. Each of the connexins can form gap junctions by themselves; however, studies have demonstrated that many cells coexpress more than one connexin isoform, giving rise to heteromeric connexons (more than two different connexins in a connexon) and heterotypic channels (a gap junction channel with different connexons), conferring further diversity in their composition and function. Connexins are named based on their predicted molecular weight (e.g., $\mathrm{Cx} 43$ is $\sim 43 \mathrm{kD}$ in size) and this nomenclature continues to be widely used. Of note, this nomenclature does give rise to confusion in some cases where homologous connexins have different names (i.e., molecular weights) in different species (Table 1; h-human; m-mouse). In the present review, we have adapted to the nomenclature decided upon at the International Gap Junction Meeting in Denmark 2007. Non-human connexins are named after their human ortholog when differing, but to ease the linking to the original literature the species dependent name is given in parenthesis. For example, mouse $\mathrm{Cx} 30.2$ is referred to as $\mathrm{Cx} 31.9(\mathrm{mCx} 30.2)$.

Connexins can be divided into five subgroups ( $\alpha, \beta, \gamma, \delta$, or $\epsilon$ ) with respect to their extent of sequence identity and length of the CL (Table 1). Connexins are then abbreviated with "GJ" for gap junction and numbered according to the order of discovery. For example, $\mathrm{Cx} 43$ was the first connexin of the a-group (GJa1) and Cx32 was the first connexin of the $\beta$-group (GJß1) (http://www.genenames.org/genefamily/gj.php).

Many studies have aimed at characterizing the high-order structure of the gap junction channel. Early work showed that a connexin hemichannel has 6-fold symmetry, with a pore in the center $(413,699)$. The initial observations were confirmed and expanded by several authors [e.g., references $(275,276,683)]$. A major step forward came from the work of Unger et al. (698). These authors used electron crystallography to solve the threedimensional structure of gap junction channels formed by $\mathrm{Cx} 43$ subunits. The assembled 
gap junction channels were observed at a resolution of $7.5 \AA$ in the membrane plane and 21 $\AA$ in the vertical direction. The study clearly showed the electron densities corresponding to 24 TM domains in a-helical order. As shown in Figure 2, they are organized as six repeats of four TM domains (labeled A-D), consistent with the notion that a connexon is formed by the oligomerization of six connexins, each with four TM domains. The images further revealed that the pore narrows from 40 to $15 \AA$ at the boundary with the extracellular gap. This narrowing [initially proposed by the low-angle $\mathrm{x}$-ray diffraction analysis of Makowski et al. (413), as well as data of Unwin and Zampighi (699)] is due to the tilting of the channel-lining TM domain (labeled "C" in the right panel of Fig. 3). Whether this structure corresponds to that of an open or a closed channel remains to be determined. The data of Unger et al. further showed that the $\mathrm{C}$ a-helix extends beyond the putative boundary of the bilayer and into the cytoplasm. If the " $\mathrm{C}$ " domain corresponds to the third TM domain this may suggest that the second half of the CL is also an a-helix, which conforms part of the vestibule of the pore. The latter would be consistent with observations of this domain using NMR (155) and with the functional mapping of this area (620). Unger et al. provided a fundamental framework for understanding the gap junction pore from a structural point of view. However, it is worth noting that the investigators needed to truncate the carboxyl terminus of $\mathrm{Cx} 43$ to improve the diffraction quality of the two-dimensional crystals. Hence, with the exception of a small fragment of the CL, intracellular regions were not characterized within this structure.

Structural analysis using x-ray crystallography has recently provided the gap junction field with the highest resolution of a gap junction channel (409). At a resolution of $3.5 \AA$, the overall structure of the Cx26 channel was determined as follows; length of the channel, approximately $155 \AA$; the extracellular gap, approximately $40 \AA$; the outer diameter of the channel at the cytoplasmic end, approximately $90 \AA$, which decreases to approximately $50 \AA$ in the extracellular portion; the inner diameter of the channel, approximately $40 \AA$ at the channel entrance, and narrows to approximately $14 \AA$ around the midpoint of the membrane region (Fig. 3A/B). The channel, which includes the 24 TM spanning domains, contains approximately $60 \%$ a-helical structure. The Cx 26 monomer is a four-helical bundle in which each pair of adjacent helices is antiparallel (Fig. 3C). TM1 and TM2 face the luminal side of the pore; however, the TM2 and the cytoplasmic half of the TM1 are not exposed to the lumen because they are covered by a short $a$-helix from the N-terminus. TM3 and TM4 are on the outside of the channel facing the lipid environment. The extracellular loop E1 contains a $3_{10}$ helix at the beginning and a short a-helix in its carboxyl-terminal half. E2, together with E1, form a short antiparallel $\beta$-sheet. Six conserved cysteine residues, three in each loop, form intramolecular disulphide bonds between E1 and E2. The intermolecular interactions between two adjoining connexons involve both the E1 and E2 domains and numerous hydrogen bonds and salt bridges help create a tight seal that isolates the channel interior to form the connection between adjacent cells. Similar to Cx43 structure (698), the $\mathrm{CL}$ and cytoplasmic tail (CT) domains were not visualized in the Cx26 electron density map. This led the authors to suggest that the channel is in an open conformation, since no obstructions through the pore were identified combined with their crystallization conditions.

Although, neither crystallographic technique was able to address the structure of the CT or CL because of the dynamic nature of these domains, these same characteristics that interfere with crystallographic techniques make NMR an ideal tool for studying them. Previous NMR studies have focused on identifying regions of structural order and binding between the CT and $\mathrm{CL}$ of $\mathrm{Cx} 43$ because these domains play a fundamental role in gap junction regulation $(155,274,647)$. For example, the CT may mediate $\mathrm{pH}$ gating in a "particle-receptor" interaction, modeled after the "ball-and-chain" mechanism of voltage-dependent inactivation (Fig. 4A) (332). A direct pH-dependent interaction between a soluble version of the $\mathrm{Cx} 43 \mathrm{CT}$ (S255-I382) and a peptide corresponding to the second half of the CL (D119-K144) (155) 
was identified. This interaction was dependent on the CL peptide forming an a-helical structure in response to the acidification (Fig. 4B). It was proposed that the second half of the CL acts as the "receptor" for the CT domain $(155,606)$. In a separate study, the soluble version of the CT domain was identified by NMR as being highly flexible and predominately disordered in structure (Fig. 4C) (648). Moreover, changes in the $\mathrm{pH}$ of the solvent caused dimerization of the Cx43CT (649), which may be a relevant step in the regulation of $\mathrm{Cx} 43$ by intracellular factors such as low $\mathrm{pH}$.

\section{Connexin structure during gap junction regulation}

Although there have been some spectacular successes in solving the structures of integral membrane proteins by xray crystallography, especially for the gap junction proteins, these structures only provide a snapshot of the protein in a single conformation. Electron crystallography, cryo-electron microscopy, and atomic force microscopy (AFM) offer alternative, albeit lower resolution, approaches to membrane protein structure determination that are not dependent on the generation of three-dimensional crystals. Over the past few years, the Sosinsky and Lal laboratories have taken advantage of these non x-ray crystallographic methods to provide novel insight into how chemical mediators of gap junction regulation (e.g., $\mathrm{Ca}^{2+}$ and $\mathrm{pH}$ ) affect the structure of $\mathrm{Cx} 43$ and $\mathrm{Cx} 26$ channels and hemichannels.

The Sosinsky laboratory used AFM to image the conformational changes of the cytoplasmic and extracellular surfaces of native Cx26 gap junction plaques in response to changes in calcium concentration (456). Calcium has long been postulated to play a crucial role in the gating of the gap junction intercellular communication by decreasing or suppressing electrical coupling (508). In the absence of calcium, the cytoplasmic surface formed a hexameric pore ( $2.8 \mathrm{~nm}$ inner diameter) protruding $1.7 \mathrm{~nm}$ from the membrane bilayer and exhibited a high degree of structural flexibility. Similar to the cytoplasmic surface, the extracellular connexon surface formed a hexameric pore exhibiting an inner diameter of 1.5 $\mathrm{nm}$ and protruded $1.6 \mathrm{~nm}$ from the lipid bilayer (Fig. 5A). When the extracellular hemichannel surface was imaged in the presence of calcium $(0.5 \mathrm{mmol} / \mathrm{L})$, the entrance narrowed significantly as evident in the reduced pore size (from 1.5 to $0.6 \mathrm{~nm}$ ) (Fig. 5B). The conformational change was fully reversible and specific amongst other divalent cations tested (e.g., magnesium). The conformational change did not alter the height nor cause a rotation of the extracellular domains. Calcium also had a profound, but different effect on the cytoplasmic surface, inducing the formation of microdomains and an increase in the plaque height. However, the cytoplasmic surface appeared too flexible to be imaged at sufficiently high resolution to assign structural changes. These observations, together with the differences in intracellular and extracellular hemichannel sensitivity to calcium concentrations (intracellular, $\mu \mathrm{mol} / \mathrm{L}$ range; extracellular, $\mathrm{mmol} / \mathrm{L}$ range), support the idea that a different gating mechanism for channel closure exists at the extracellular surface from the one that occurs at the cytoplasmic surface.

A different mechanism underlying $\mathrm{Cx} 26$ channel closure was observed in response to alterations in $\mathrm{pH}$ (772). High-resolution imaging by AFM of Cx26 hemichannels revealed that the pore was closed in response to acidification, but only in the presence of an aminosulfonate buffer (e.g., HEPES). Aminosulfonate compounds acts as modulators of the Cx26 channel (41). The overall diameter of the hexameric hemichannel did not change, yet the depth of the channel decreased concomitant with an increase in the width of the connexon lobes under the more acidic conditions. For example, at $\mathrm{pH} 7.6$, the channel entrance diameter was $1.7 \mathrm{~nm}$, while at $\mathrm{pH} 6.0$, the diameter was $0.6 \mathrm{~nm}$ and there was an approximately $6.5^{\circ}$ rotation in the hemichannel lobes. These results support the model of a physical gate close to the extracellular surface, not only because the pore diameter decreases upon acidification, but also because its depth is shallower. 
More recently, the Sosinsky laboratory has reported the electron crystallographic structure of a human Cx26 mutant (M34A) (490) previously shown to cause prelingual nonsyndromic hereditary deafness [M34T (308)]. The mutation expressed higher quantities of protein than wild-type (wt) Cx26. Interestingly, the Cx26 mutant was purified as hemichannels; however, during the crystallization process, full Cx26 gap junction channels were formed. This indicates that hydrophobic interactions at the extracellular domains may drive hemichannels to redock into dodecameric channels. The three-dimensional structure was determined to a resolution of $10 \AA$ in the $x-y$ plane. A comparison was made between the Cx26 M34A mutant ( $\beta$-subgroup) and the $\mathrm{Cx} 432$ 263T truncation mutant (a-subgroup) and the structure of these two connexin isoforms were very similar (698). Also, similar to the Cx43 263T truncation mutant and Cx26 crystal structure, the resolution of the cytoplasmic and extracellular domains was insufficient to assign these segments and the polypeptide boundary to specific sequences within the TM domain. The three-dimensional structure also displays a prominent density in the pore of each hemichannel, suggesting that a plug physically blocks the channel within the membrane. The likely candidates for the plug are the cytoplasmic N-terminal, CT, or CL domains.

AFM has also been utilized to examine the threedimensional molecular surface topology of nontruncated $\mathrm{Cx} 43$ hemichannels reconstituted in lipid membranes (677). The images showed that the hemichannels fell within two groups that differed in height. The groups were identified as the extracellular surface facing-up, with a height of $1.3 \mathrm{~nm}$ from the membrane surface and the intracellular surface facing-up, with a height of $4.7 \mathrm{~nm}$. The latter is consistent with the longer protruding CT domain and allowed for a clear distinction of the sidedness of the images because each hemichannel has an equal probability of facing-up or down. A detailed analysis of the calcium-sensitive conformational changes was performed with the $\mathrm{Cx} 43$ hemichannels. In calcium-free buffer, AFM images of the extracellular face showed a well-defined donut-like structure consisting of six subunits and a central pore-like depression. The pore diameter was $2.5 \mathrm{~nm}$ with a depth of $0.8 \mathrm{~nm}$. This pore size was not changed in the presence of magnesium or nickel; however, calcium was able to close the channels in a concentrationdependent manner. The channels were completely closed at 1.8 $\mathrm{mmol} / \mathrm{L}$ calcium (pore diameter $-1.8 \mathrm{~nm}$ ), while $73 \%$ and $26 \%$ of the channels were closed at 1.4 and $1 \mathrm{mmol} / \mathrm{L}$, respectively. Only two-states $(2.5$ and $1.8 \mathrm{~nm})$ were observed, suggesting that a single hemichannel switches between open and closed states in an all-ornone manner, and the open/closed probability is calcium concentration dependent. This is consistent with previous functional studies of hemichannels and their physiological role $(159,280,514)$.

The open and closed channel diameters for $\mathrm{Cx} 43(2.5$ and $1.8 \mathrm{~nm})$ are similar to those predicted from the electron microscopy study by Unger et al. (698), but quite different when compared with the open and closed diameters $(1.5$ and $0.6 \mathrm{~nm}$ ) for isolated Cx26 gap junction channels (456). Also, Muller et al. (456) showed that $\mathrm{Cx} 26$ hemichannels close at a lower calcium concentration than $\mathrm{Cx} 43$ hemichannels ( 0.5 and $1.8 \mathrm{mmol} / \mathrm{L}$, respectively), suggesting this difference in calcium sensitivity may be related to the functional differences between the two hemichannels.

Interfacial energy maps were used to determine the hydrophobic versus hydrophilic surfaces, both on the Cx43 extracellular loops, as well as the pore mouth (677). In the closed state, the extracellular loops are tightly packed and in the open state, the extracellular loops are at least partly unfolded (a larger pore, a slightly larger outer diameter, and well-resolved extracellular loops). The hemichannel opening is associated with a significant increase in the interfacial energy in the pore mouth region $\left(0.118 \mathrm{~J} / \mathrm{m}^{2}\right.$ for the closed and $0.163 \mathrm{~J} / \mathrm{m}^{2}$ for the open channels). Such an increase would indicate the presence of hydrophobic surfaces exposed at the pore vestibule. The data indicate that refolding of the extracellular loops 
exposes their hydrophobic domains in a calcium-dependent manner and that the extracellular loops in nonjunctional hemichannels are structurally different when compared with those in the gap junctional hemichannels.

Based on the "particle-receptor" model for $\mathrm{Cx} 43$ channel gating, movement of the CT domain controls channel permeability by interacting with the CL domain to physically occlude the pore $(155,274,454)$. To test this hypothesis, the Lal laboratory used AFM-based single-molecule spectroscopy with antibody modified AFM tips and connexin mimetic peptide-modified tips to examine the flexibility of Cx43 CT domain and extracellular loop domains, and to estimate the energetics of their movements (387). For these experiments, AFM tips were linked via a flexible spacer to either a CT specific antibody (anti-CT $252-270$ or anti-CT $360-382$ ) or to a connexin mimetic peptide (called GAP26, which binds to the extracellular loop), and subsequently binding to $\mathrm{Cx} 43$ hemichannels reconstituted in a lipid bilayer was measured. The observed GAP26-Cx43 interaction showed very little, if any, molecular stretching of the Cx43 extracellular loop. However, a significantly larger

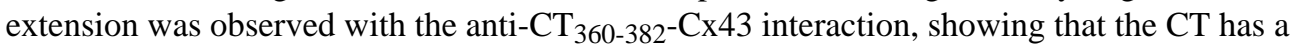
large degree of flexibility. As expected, the anti- $\mathrm{CT}_{252-270}-\mathrm{Cx} 43$ interaction caused a shorter extension because its interaction site is located in the middle portion of the flexible $\mathrm{Cx} 43 \mathrm{CT}$ domain and thus closer to the membrane. As previously observed, $1.8 \mathrm{mmol} / \mathrm{L}$ calcium closes Cx43 hemichannels (677). To further examine the role of the Cx43 CT domain, calcium was added into the anti- $\mathrm{CT}_{360-382}-\mathrm{Cx} 43$ force measurement medium. The specific rupture events (which indicate binding between the antibody and Cx43 CT) frequently detected under calcium-free conditions were significantly diminished in the presence of 1.8 $\mathrm{mmol} / \mathrm{L}$ calcium, a process that was fully reversible. Hemichannel closing at $1.8 \mathrm{mmol} / \mathrm{L}$ calcium is believed to introduce conformational changes in the $\mathrm{Cx} 43 \mathrm{CT}$ domain, which results in steric hindrance preventing binding. Altogether, the stretch length, the presence of antibody-CT domain interaction at $0 \mathrm{mmol} / \mathrm{L}$ calcium, and the lack of one at $1.8 \mathrm{mmol} / \mathrm{L}$ calcium, strongly support calciumdependent conformational changes of the CT domain and the "ball and chain" model of hemichannel gating.

\section{Permeability of gap junction channels}

Traditionally, gap junctions have been characterized as nonspecific pores between cells passing molecules up to $1 \mathrm{kDa}$ in molecular mass, and expectation was that the pore diameter would be the primary selectivity determinant among the different connexin isoforms. Since then, a variety of approaches have been used to investigate permeability properties of connexin channels. Studies involving measurement of unitary conductance, ion selectivity, and permeability to fluorescent tracers of various sizes, charges, and chemistries, have revealed a wide variety of pore properties. No atomic ions are large enough to be excluded and homotypic gap junctions of $\mathrm{Cx} 43, \mathrm{Cx} 40, \mathrm{Cx} 37$, and $\mathrm{Cx} 45$ shows similar selectivity to monovalent cations (e.g., $\mathrm{K}^{+}$and $\left.\mathrm{Na}^{+}\right)(32,716,717,733)$; however, the charge selectivities range from a slight anion preference (Cx32) to a high cation selectivity (Cx40 and Cx43) $(32,667,733)$. The electrophysiological properties of macroscopic coupling are determined by the biophysical properties of the underlying channels. Channel behavior is complex usually with a main open state, as well as states of lower conductance. The unitary conductance of gap junction channels depends on the connexin isotypes; Cx36 channels display a unitary conductance of $14 \mathrm{pS}$ (674), whereas Cx37 form channels with a very high electrical conductance of $300 \mathrm{pS}$ (718). The conductance of cell-to-cell channels can in most cases be calculated from the conductance of the individual hemichannels under the assumption that the channels work as two resistors in series.

The initial use of a range of fluorescent tracers and uncharged molecules to study gap junction permeation provided significant information regarding the pore diameter limitation of $\mathrm{Cx}$ channels (suggested ranking: $\mathrm{Cx} 43>\mathrm{Cx} 32>\mathrm{Cx} 26>\mathrm{Cx} 37>\mathrm{Cx} 46$ and $\mathrm{Cx} 43>\mathrm{Cx} 40$ ) 
(248). Though, fluorescent tracers became useful to report the existence and extent of junctional communication (e.g., use of Lucifer Yellow), they provide limited information regarding the nature of the permeability pathway itself. Important as the use of nonbiologic tracers have been in the estimation of pore diameters, these tracers convey little information on the ability of connexins to transfer biological molecules such as metabolites and second messengers. Although the literature on permeation of biological molecules is limited, important efforts have focused on the characterization of the permeability of connexin channels to endogenous cytoplasmic molecules. This is not a simple task due to the existence of more than 20 connexin isoforms, with different pore properties, that can almost all form heteromeric channels. Additionally, permeability properties of a given channel are expected to be modulated by reversible posttranslational modification (e.g., phosphorylation, see section on posttranslational modifications) or protein-protein interactions. Each answer will therefore be specific to a particular structural and biochemical channel, at a specific time. Measuring the flux of biological molecules poses many problems that make a reliable quantification difficult. Often the molecules in question are subject to cellular metabolism and in the case of second messengers, the messengers can affect coupling by activation of downstream cascades. In both cases, transfer can be grossly overor underestimated. Recent reviews summarize extensive information regarding the permeation by cytoplasmic molecules through connexin channels $(248,249)$. As previously established, most of the studied gap junction channels (homomeric Cx26, Cx32, and Cx43 and heteromeric Cx26/ $\mathrm{Cx} 30$ or $\mathrm{Cx} 32$ ) are permeable to second messengers and metabolites, such as ATP or ADP, $\mathrm{Ca}^{2+}$, cAMP, $\mathrm{IP}_{3}$, glutamate, glutathione, and of interest to siRNA. However, connexin isoforms can influence the gap junctional permeability. For example, $\mathrm{Cx} 43$ channels have a 100- to 300-fold higher selectivity for ATP over those of $\mathrm{Cx} 32$, while glutamate, glutathione, and ADP show a 10- to 20-fold preferential permeability through $\mathrm{Cx} 43$ channels, whereas adenosine is 10-fold more permeable through Cx32 (219). These studies demonstrate the differences in intercellular transfer of natural permeants among the different connexins. However, the physical basis of this selectivity remains unclear, as there are no evident correlations between metabolite properties and their permeability through different connexin channels.

Despite the limited information on permeation of endogenous molecules, it is quite clear that large differences occur between different connexin species. These differences may explain the need for multiple connexin types to regulate communication during development and signaling events. That the differences in permeation are of physiological importance are exemplified in mouse models where connexin genes have been exchanged with another connexin. In the case of $\mathrm{Cx} 43$ exchange to $\mathrm{Cx} 31$ or $\mathrm{Cx} 26$ leads to functional and anatomical defects $(748,782)$, whereas exchange to $\mathrm{Cx} 32$ or $\mathrm{Cx} 40$ has little or no consequence (524). The effects may be very tissue specific as shown in a recent study, where neuronal specific exchange of Cx45 with Cx36 did not affect signaling in the retina. However, cardiac or general exchange did lead to both enlarged hearts and conduction abnormalities and were embryonic lethal (185). Thus, the specific differences in connexin properties can have profound physiological consequences.

\section{Compatibility of connexin types in channel formation}

Connexins are somewhat promiscuous in the sense that different connexins can combine to form channels with unique properties. Channels with diverse connexin content are classified on the basis of how the connexins are mixed (Fig. 6). Individual connexons (hemichannels) are termed homomeric when they contain only one connexin type and heteromeric if they contain different connexins. When identical connexons form cell-cell channels they are termed homotypic. In some cases, cells with connexons of different connexin composition form channels, which are then termed heterotypic. This gives rise to four possible 
combinations of homo/heteromeric and homo/heterotypic channels. Given the 21 connexins in humans and the fact that connexons contain six connexins, this leads to an explosion of possible combinations. However, not all connexins are compatible in forming functional channels and secondly not all combinations of connexins are expressed in the same or neighboring cells.

Heterotypic combinations of homomeric channels have been studied extensively and are physiologically relevant when cells expressing different connexins couple. Connexin of the $\alpha$ - and $\beta$-groups are more likely to form channels with other members of their own group. For example, in the a group, $\mathrm{Cx} 37$ forms heterotypic channels with $\mathrm{Cx} 40$ (747) and $\mathrm{Cx} 43$ (64), but not with theßconnexins $\mathrm{Cx} 26$ and Cx32 (747). On the other hand, $\mathrm{Cx} 37$ does not form channels with the a-connexins Cx46 and Cx50 (747), so any given combination of interest needs to be tested. A more exhaustive review of possible combinations can be found in reference (763). The functional properties of heterotypic channels are often similar to that expected of two connexons in series and will be dealt with in the section on voltage gating.

Coexpression of different connexins occurs in many cell types as, for example, in the skin, where $\mathrm{Cx} 31, \mathrm{Cx} 26, \mathrm{Cx} 43, \mathrm{Cx} 32, \mathrm{Cx} 40, \mathrm{Cx} 30$, and $\mathrm{Cx} 45$ are expressed at the protein level (144). The study of heteromeric channels is complicated because even if coexpression results in channels/coupling with altered properties, it is difficult to prove that connexins actually incorporate into the same channel. Conversely, even if heteromeric channels are formed, they will exist together with homomeric channels. The relative proportions of hetero- and homomeric connexons will be determined by the probability of hetero- and homomeric oligomerization. In the atrium of the heart, $\mathrm{Cx} 40$ and $\mathrm{Cx} 43$ are coexpressed and studies show that they most likely form heteromeric channels $(256,702)$. However, the alterations in macroscopic coupling properties can largely be described by the behavior of homo- and heterotypic channels and it has been concluded that the heteromeric channels are of little importance (702). In contrast, coexpression of $\mathrm{Cx} 40$ and $\mathrm{Cx} 43$ significantly increases the sensitivity to $\mathrm{pH}$-dependent gating (233). Furthermore, it has been shown that the CTs of Cx40 and Cx43 can interact with each other and with each other's CL, suggesting that interactions within connexins in heteromeric channels are responsible for the shift in $\mathrm{pH}$ dependence (57).

\section{Gating of Connexins}

The term gating is often used to describe changes in channel opening and closing, that is, a conducting channel becomes either physically available or unavailable (271). Electrical coupling via gap junctions, expressed by the macroscopic conductance $(\mathrm{Gj})$, is determined by three factors: the number of channels present at the plasma membrane $(N)$, the open probability (Po), and the unitary conductance of the channel $(\mathrm{\gamma})$, where $\mathrm{G}_{\mathrm{j}}=N^{*} \mathrm{P}_{\mathrm{o}} * \mathrm{\gamma}$. The number of channels residing in gap junctional plaques is determined by the expression, trafficking, and degradation of connexins, which is covered in a later section. Here, regulation of $\mathrm{P}_{\mathrm{o}}$ and $\mathrm{\gamma}$ of channels residing in gap junctional plaques will be discussed. Availability is determined by the $\mathrm{P}_{\mathrm{o}}$ of the channels but connexin channels also exhibit several conductance levels, the size of which can be regulated, thence unitary conductance must be taken into consideration. As we shall discuss, channel activity is tightly regulated in response to changes in voltage, calcium concentration, $\mathrm{pH}$, phosphorylation, and protein interactions.

\section{Voltage gating}

Gating by the transjunctional voltage-Gap junction conductance can be regulated by transjunctional voltage $\left(\mathrm{V}_{\mathrm{j}}\right)$ and for some connexins also by the transmembrane voltage $\left(\mathrm{V}_{\mathrm{m}}\right)$ (for reviews see references 72 and 227). There are 21 different connexins in humans 
and each has distinct gating properties. When gap junctional currents are investigated by imposing a voltage gradient, the elicited current inactivates over time in a voltage-dependent manner (see Fig. 7). The initial current is largely independent of voltage, whereas the steady-state current decreases with increasing voltage gradients. The inactivation can be described by the fractional maximum conductance $G_{\max }$, which expresses the conductance relative to the conductance at $V_{j}=0$; the fractional minimum conductance $G_{\min }$, which expresses the inactivation at large $\mathrm{V}_{\mathrm{j}}$; and the $\mathrm{V}_{\mathrm{j}}$ at which half maximal inactivation occurs $\left(\mathrm{V}_{1 / 2}\right)$. The parameters can be determined by fitting a Boltzmann function to the steady-state current divided by the initial current as a function of $V_{j}(250)$. This type of voltagedependent behavior is reported for all connexins investigated with variation in the degree, speed, and sensitivity of inactivation. $V_{1 / 2}$ indicates the sensitivity to $V_{j}$ and varies from around $100 \mathrm{mV}$ in $\mathrm{Cx} 36$ (5) to around $10 \mathrm{mV}$ in $\mathrm{Cx} 45$ (24), the most sensitive connexin. The effect of voltage-dependent inactivation is also very variable with a $G_{\min }$ of 0.5 in perch Cx35 (5) to 0.1 in Cx45 (24). Table 2 shows values for selected connexins, an exhaustive list can be found in reference 227 .

The physiological role of voltage gating is largely unexplored, but may for some connexins like $\mathrm{Cx} 43$ overlap with the chemical gate, since truncation of the $\mathrm{CT}$ in this connexin eliminates both sensitivity to uncoupling at low $\mathrm{pH}$ and voltage gating $(455,559)$. For many connexins the voltage gradient required to close the channels is unlikely to occur under physiological conditions even in excitable tissue, but for connexins that are highly sensitive to voltage, functional roles have been proposed. $\mathrm{Cx} 45$ is the most sensitive connexin and is expressed in Purkinje fibers in the heart. These fibers are responsible for conducting action potentials from the conduction system to the working myocardium, whereas the myocytes of the myocardium express only Cx43, which is less sensitive. Cx45 closes when its cytoplasmic face becomes negative relative to the neighbor cell and thus it has been proposed that this prevents retrograde conduction from the myocardium to the conduction system. More recently, it has also been shown that even relative small stable voltage gradients between cells may increase the sensitivity of the gate (498).

Measurements on gap junctional channels in insect cells have shed light on the mechanisms underlying $\mathrm{V}_{\mathrm{j}}$ gating. Gap junction channel recordings in insect cells provided evidence that connexins have several conductance states. In these studies, cells were patch-clamped and pushed into contact to observe the formation of electrical coupling. The opening of the first channel from the closed to the main open state was slow and appeared to be composed of a number of smaller transitions. The open channel exhibited fast transitions from the main open state to a substate with a conductance approximately $20 \%$ of the main open state. This transition was voltage dependent, and in contrast to the slow opening to the main state, the transitions to and from the substate (often referred to as the residual state) were much faster $(73,74)$. Although the previous observations were made in insect cells that express innexins rather than connexins, similar conductance steps have been detected in the mammalian connexins, for example, Cx37 (718), Cx43 (451), and Cx40 (71).

Based on the speed of transition, the movement to and from the closed state is termed slow gating (or loop gating), and the faster transition between the main open and residual states is called fast gating. Each hemichannel of a cell to cell channel contains its own gates, which operate in series and determine whether the channel is closed, full open, or in its residual state. For a number of connexins, macroscopic $V_{j}$ gating is probably related to channel closure by the fast gate, because the fraction of the residual current correspond to the relation between the conductance of the full open and the residual state (72). However, during prolonged large $V_{j}$ gradients the slow gate may also close (70) in which case the concerted voltage dependence will be affected by both gates. 
Each hemichannel contains voltage gates, which may be closed or open depending on the relative polarity (see Fig. 7). In homotypic channels, the relative polarity, at which a channel closes, cannot be determined, but by pairing channels with different gating properties, it is possible to determine the polarity of gating. In the following, gating at negative polarity means that the gate closes when the cytosolic side of the hemichannel becomes negative, and positive gating occurs at relatively positive potentials. In all cases, it seems that slow gating occurs at negative polarity (72). Fast gating on the other hand may occur at either polarity depending on the connexin type, for example, Cx26 closes at positive polarity and Cx32 at negative (25). Gating at positive polarity has been demonstrated for Cx26, Cx30, Cx37, Cx40, Cx46, and Cx50, whereas Cx31, Cx31.9 (mCx30.2), Cx32, Cx43, and Cx45 gate at negative polarity (227).

Determinants of $\mathbf{V}_{\mathbf{j}}$ gating - $T$ he $\mathrm{V}_{\mathrm{j}}$ gating of connexins involves several parts of the molecule and so far the NT, TM1, CL, and CT have been implicated. Cx26 and Cx32 have opposite gating polarity and therefore heterotypic channels only close at one polarity (when Cx26 is relatively positive the cytoplasmic side of $\mathrm{Cx} 32$ will be negative and therefore both gates will close), in contrast homotypic $\mathrm{Cx} 26$ or $\mathrm{Cx} 32$ channels gate symmetrically. Verselis et al. showed that exchange of the NT and TM1 of Cx26 with that of Cx32, resulted in channels that gated asymmetrically with $\mathrm{Cx} 26$ channels and symmetrically with $\mathrm{Cx} 32$ channels. In other words, the NT and TM1 completely determined the gating (725). The polarity of gating was entirely determined by the second amino acid of the NT, which is uncharged in Cx32 (N2) and negatively charged in Cx26 (D2). Mutation of N2 in Cx32 to a negatively charged amino acid reversed the polarity of gating to positive, whereas a positive amino acid had no effect. Conversely, changing D2 of Cx26 to either an un- or positively charged amino acid, changed the gating polarity of $\mathrm{Cx} 26$ to negative (725). In accordance with this, connexins with positive polarity gating such as Cx26, Cx30, Cx37, Cx40, Cx46, and $\mathrm{Cx} 50$ are negatively charged at the NT. For channels gating at negative polarity the picture is less clear; $\mathrm{Cx} 45$ conforms to the rule by having a relatively positive NT. In contrast, the charge pattern of $\mathrm{Cx} 31$ and $\mathrm{Cx} 43$ resemble that of $\mathrm{Cx} 26$, but they gate at opposite polarity showing that other amino acids must interfere in the determination of gating polarity. For a thorough review of the effects of amino acid substitutions in the NT and TM1, see reference 227 .

Voltage gating also depends on the $\mathrm{CT}$ and its truncation eliminates fast $\mathrm{V}_{\mathrm{j}}$ gating of $\mathrm{Cx} 32$ (559), Cx40 (9), and Cx43 (449, 559) (see Fig. 8). Furthermore, interference with CT structure by attaching aequorin or green fluorescent protein (GFP) also prevents fast gating (70, 417). However, the rule of CT involvement is not universal since CT truncation of Cx50 (756) or the attachment of EGFP to Cx47 (675) does not affect gating. It has been proposed that the CT closes the channel by a "particle-receptor" mechanism similar to the "ball and chain" mechanism suggested for fast inactivation of Na channels (11). In the case of connexins, the $\mathrm{CT}$ acts as a gating particle, which upon binding to its receptor closes the channel to its residual state. Truncation of the $\mathrm{CT}$ of $\mathrm{Cx} 43$ eliminates fast gating without affecting slow gating and in support of the receptor-particle model, expression of the $\mathrm{Cx} 43$ $\mathrm{CT}$ as a separate entity restores fast gating (449). Similar findings were reported for $\mathrm{Cx} 40$ and interestingly concatenation of the $\mathrm{Cx} 43-\mathrm{CT}$ to the truncated $\mathrm{Cx} 40$ restored gating to the residual state (9).

The receptor to which the CT binds is probably located in the second half of the CL, also referred to as the L2 region. Duffy et al. showed that the CT binds the L2 region in a pHdependent manner (155) and supporting its role in fast gating, addition of L2 peptide to the cell interior via the pipette solution prevented fast $\mathrm{V}_{\mathrm{j}}$ gating (606); also mutation of histidine to glutamate at position 142 reduced the probability of the residual state and inhibited fast gating (620). 
Gating by transmembrane voltage $-V_{m}$ is sensed by connexin channels across the pore in the membrane and especially in the extracellular part of the channel. $\mathrm{V}_{\mathrm{m}}$ dependence is pronounced in innexin expressing insect cells, but less common in connexins (72). Cx43 was shown to be gated by $\mathrm{V}_{\mathrm{m}}$ with depolarization closing the channels. The gating depended on a region at the border of the TM4 and the CT, since truncation at amino acid 242 abolished Vm gating, whereas truncation at 257 did not (558). In the case of $\mathrm{Cx} 43$, uncoupling by cell membrane depolarization may be a mechanism to protect neighboring cells from the electrotonic influence of pathologically depolarized cells.

\section{Chemical gating}

Connexins are also gated by the chemical composition of the intracellular milieu, especially by $\mathrm{pH}$ and $\mathrm{Ca}^{2+}$. This type of gating has profound physiological implications and uncoupling by these agents have been proposed to have both beneficiary/protective effects (196) and deleterious effects (13). Chemical gating may also occur due to application of exogenous chemicals and pharmacological agents, which is covered in the section on pharmacology of gap junctions.

pH-dependent gating-Intracellular acidification uncouples gap junction channels composed of Cx26 (41), Cx32 (742), Cx38 (735), Cx43 (391), Cx46 (162), Cx50 (ovine $\mathrm{Cx} 49)(162)$, and $\mathrm{Cx} 62$ (mCx57) (499). The set point for half maximal uncoupling by $\mathrm{pH}$ is variable and the number of channels active at physiological $\mathrm{pH}$ depends highly on the connexin type involved. In the case of $\mathrm{Cx} 46$ and $\mathrm{Cx} 50$ most channels will be active at $\mathrm{pH}$ 7.2 (162), whereas only $1 \%$ of all $\mathrm{Cx} 62$ (mCx57) channels are open at pH 7.2 (499). Therefore, the effect of acidification or alkalinization on intercellular coupling will differ between tissues relative to the connexin expressed. $\mathrm{Cx} 43$ is the most well-studied connexin with regard to $\mathrm{pH}$ and much is known about its regulation and the mechanisms involved. $\mathrm{Cx} 43$ is mainly in the open configuration at $\mathrm{pH} 7.2$ (163). As described for $\mathrm{V}_{\mathrm{j}}$-dependent gating, there is good evidence that $\mathrm{pH}$ gates $\mathrm{Cx} 43$ by a particle-receptor mechanism and truncation of the CT makes the channels insensitive to changes in $\mathrm{pH}$ (454). The sensitivity to $\mathrm{pH}$ is reintroduced by coexpression of the $\mathrm{CT}$ with the truncated $\mathrm{Cx} 43$ and furthermore Cx43-CT expression also increases the $\mathrm{pH}$ sensitivity of Cx32 (454). The receptor-binding site of the CT is located in the second half of the CL (amino acids 119-144, termed L2) (155) and increased binding is induced by structural changes (for references see section on structure of connexins). Another piece of evidence comes from the identification of peptides with the ability to bind the CT. One such peptide, RXP-E, binds to the Cx43-CT and partially prevents uncoupling by acidification and heptanol (621). The section on pharmacology contains a detailed description of the further development of these compounds.

$\mathrm{Ca}^{2+}$-dependent gating-Increasing intracellular $\mathrm{Ca}^{2+}$ uncouples gap junctions in a number of tissues. This is often considered a protective mechanism, which will prevent dying cells from destroying their neighbors. However, the range of $\mathrm{Ca}^{2+}$ concentration needed for uncoupling varies greatly between studies and there is little consensus on the amount of $\mathrm{Ca}^{2+}$ needed for uncoupling (508). Synergistic effects between $\mathrm{Ca}^{2+}$ and $\mathrm{pH}$ are found in some studies $(76,477)$. Examining the effects of changes in $\mathrm{Ca}^{2+}$ and $\mathrm{pH}$, the effects of $\mathrm{pH}$ on intracellular $\mathrm{Ca}^{2+}$ and vice versa, as well as the fact that the buffer capacity of the often used $\mathrm{Ca}^{2+}$ buffer EGTA is very sensitive to changes in $\mathrm{pH}$ (363) may be a confounding factor. The effect of $\mathrm{Ca}^{2+}$ is suggested to act via calmodulin and inhibitors of calmodulin prevents uncoupling in a number of cell types (for review see reference 508). The data is supported by expression of calmodulin mutants with increased affinity for $\mathrm{Ca}^{2+}$, showing that increased affinity of calmodulin also increases $\mathrm{Ca}^{2+}$ sensitivity of $\mathrm{Cx} 32$ (509). Cx32 has been shown to contain two calmodulin binding domains (685). With an increase in 
$\mathrm{Ca}^{2+}$, calmodulin is believed to either physically block the channel or close the channel through a conformational change in $\mathrm{Cx} 32$. This may be mediated by transdomain bridging of calmodulin, where calmodulin interacts with the NT and the CT binding sites simultaneously (150). A role for calmodulin in gating has also been suggested for $\mathrm{Cx} 36$ (75), Cx43 (402), Cx44 (783), and Cx45 (510). In the case of Cx43, calmodulin is believed to bind the CL in a region overlapping with the L2 binding site for the CT (784), which may augment channel closure by the particle-receptor mechanism.

\section{Control of Connexin Expression in the Cell Membrane}

Besides direct regulation of gap junction channel function, the functional intercellular coupling is determined by the type of connexin and the number of channels present within gap junction plaques. Different cell types express different connexins, and both the composition and quantities may vary over time. Tissue and time specific control of connexins is also achieved by regulating mRNA levels, protein synthesis, trafficking, and degradation. In the following, mechanisms, which regulate the expression of connexins and gap junctions in the cell membrane, are reviewed and selected examples of physiological functions are given.

\section{Connexin transcription}

Transcription factor control-The expression of connexins is determined by the transcription factors, which govern the transcription of their genes. The gene structure of connexins consists of two or more exons. The entire coding region is generally placed in the last exon, which is preceded by uncoding exons (496). Exceptions to the rule are Cx36, Cx40.1 (mCx39), and Cx62 (mCx57), in which the coding region spans two exons (642). The connexin transcripts can vary in their $5^{\prime}$ untranslated region (5'UTR) due to differences in the transcription of the preceding non-coding exons. In some cases, these differences are linked to the activation of different promoter regions. For example, rat and human Cx32 contains two 5'UTR exons (435), each of which are preceded by a separate promoter region (263). In liver cells, the first promoter region is activated by hepatocyte nuclear factor-1 resulting in one transcript (324), whereas in Schwann cells, activation of the second promoter by Sox-10 yields a different transcript (54). In the case of mouse $\mathrm{Cx} 43$, the situation is considerably more complex since three promoter regions and alterative splicing produce nine different transcripts. The distribution of transcripts varies between and within tissues and since the translation efficacy of the transcripts varies greatly, it is likely that differences in protein levels are partly governed by the nature of the transcript 5'UTR (515).

Transcription of most connexins is regulated by a combination of ubiquitous and tissue specific transcription factors. An interesting example is the heart, where $\mathrm{Cx} 40, \mathrm{Cx} 43$, and $\mathrm{Cx} 45$ are differentially expressed in the chambers and conduction system. In the atria $\mathrm{Cx} 40$ and $\mathrm{Cx} 43$ are co-expressed, whereas $\mathrm{Cx} 43$ is the sole connexin of the ventricular myocardium (613). Expression of $\mathrm{Cx} 40$ is driven by the cardiac specific transcription factors Tbx5 (65) and GATA4 (385) together with the ubiquitous Sp1 (45). In early development, $\mathrm{Cx} 40$ is expressed in the developing ventricles up to 14 days postcoitum, but absent from the ventricle on day 19 (139). A possible explanation for the disappearance of $\mathrm{Cx} 40$ is that the ventricle specific transcription factor HRT2 downregulates $\mathrm{Cx} 40$ by repressing the transcription of Tbx5 (325) and GATA4 (304). In accordance with this hypothesis, knockout of HRT2 results in left ventricular expression of atrial genes including Cx40 (325). In the conduction system, $\mathrm{Cx} 40$ and to a lesser extent $\mathrm{Cx} 43$ are expressed only in the more distal parts (613). The reason may partly be that $\mathrm{Cx} 40$ and $\mathrm{Cx} 43$ transcription is repressed by $\mathrm{Tbx} 2$ (90), Tbx3 $(55,278)$ and in the case of $\mathrm{Cx} 43$ by Nkx2.5 (676). Thus, it seems that much of the specificity of connexin expression in the heart is governed by a combination of tissue specific transcription activators and repressors. 
Connexins are also under the influence of ubiquitous transcription factors and may be regulated dramatically within very short time frames in important physiological functions. An example is the $\mathrm{Cx} 43$ upregulation in the uterus during labor, which is considered essential for the synchronization of forceful coordinated contractions (203). The expression level of $\mathrm{Cx} 43$ is low during pregnancy, possibly due to the action of progesterone, which inhibits Cx43 transcription (491) by downregulating AP-1 (438) and upregulating the transcription repressors p54nrb (151) and ZEB1 (551). At the time of labor, a combination of hormonal activation and stretch of the myometrium increases the expression AP-1 proteins of the Fos and Jun families and together they form an AP-1 transcription factor that activates the $\mathrm{Cx} 43$ promoter $(513,521,628,754)$. This increases the $\mathrm{Cx} 43$ protein level and the formation of gap junction plaques. Once coupling is high and contraction is stimulated, synchronous contraction occurs. The importance of $\mathrm{Cx} 43$ upregulation is demonstrated by the delayed parturition in mice with a smooth muscle specific knockout of $\mathrm{Cx} 43$ (152) and in mice carrying a dominant negative oculodentodigital dysplasia mutation in Cx43 (684). Cx43 is also upregulated in the human uterus at term $(89,95)$. One study has addressed the role of $\mathrm{Cx} 43$ in human parturition, where the $\mathrm{Cx} 43 \mathrm{mRNA}$ and protein levels was reduced in uterine biopsies from women experiencing prolonged labor compared to women giving normal birth (99).

Epigenetic regulation-Besides direct regulation via transcription factors, connexin mRNA/expression levels are also regulated by histone acetylation, DNA methylation, and micro-RNAs. Much of the evidence for the epigenetic regulation arises from broad inhibition of the enzymes involved, with subsequent investigation of mRNA levels, protein levels, and/or functional coupling. When interpreting the results, it is important to remember that general inhibition of epigenetic mechanisms may regulate connexin expression and function by changing the expression of other proteins. An example of this is the direct acetylation of connexins (728).

Histone acetylation: DNA is wrapped around histones in a structure called the nucleosome. The nucleosomes are involved in the packing of DNA and the tightness of this packing regulates gene transcription. Histone function is regulated by covalent modifications such as acetylation. Histone acetylation reduces the interaction between nucleosomes, loosens the chromatin structure, and promotes transcription. Acetylation is mediated by histone acetyltransferases (HATs) and deacetylation by histone deacetylases (HDACs). Histone acetylation is reported to affect several connexins in different cell types (728). Inhibition of HDACs was first shown to increase the amount of $\mathrm{Cx} 43$ (481) and later demonstrated for Cx36 (416) and Cx32 (729).

HDAC inhibition increases the transcription of genes that are repressed due to acetylation. Cx36 is dominantly expressed in neuronal tissue (351). Transcription of neuronal genes is repressed in other cell types by RE-1 silencing transcription factor (REST)-mediated recruitment of HDACs to the target genes. Haefliger and coworkers showed that the regulatory region of the $C \times 36$ gene contains a REST binding element, which recruits REST and silences Cx36 transcription in HeLa cells (416). Another study showed that HDAC inhibition or expression of a REST mutant that activates transcription, upregulated Cx36 in non-neuronal cells but not in neuronal (277), consistent with the idea that Cx36 is expressed in neurons mainly due to the lack of REST-mediated repression.

HDAC inhibitors also inhibit deacetylation of cytosolic proteins and may thereby affect gap junction function independently of transcription. This is seen in $\mathrm{mdx}$ mice, a genetic cardiomyopathy model, where $\mathrm{Cx} 43$ is redistributed from the intercalated disc to the lateral membranes (102). This condition is rescued by acetylase inhibition, whereas HDAC inhibitors induce the lateralized phenotype in control mice (102). The changes are related to 
Cx43 localization rather than to its expression and demonstrate that changes in coupling after interference with acetylation are not necessarily related to the regulation of connexin gene transcription.

DNA methylation: Methylation of cytosines is a mechanism of gene regulation and hypermethylation represses gene expression. The $\mathrm{Cx} 32$ promoter is methylated in liver epithelium, which expresses $\mathrm{Cx} 43$ but not $\mathrm{Cx} 32$, whereas hepatocytes, which express $\mathrm{Cx} 32$ but not $\mathrm{Cx} 43$, are methylated in the $\mathrm{Cx} 43$ promoter (519). These results suggest that methylation plays a role in normal physiological regulation of connexin expression; however, most of our knowledge about regulation by methylation arises from studies of cancer. Cancer is often associated with reduced levels of connexin expression, which is believed to play a role in the lack of growth regulation. In HeLa cells, clones that do not express $\mathrm{Cx} 43$ have higher growth rates and treatment with a demethylating agent leads to reexpression of Cx43 and increased growth control (313). Methylation of the Cx43 promoter reduces Cx43 levels in nonsmall cell lung cancer by interfering with AP-1 binding (87) and downregulation of $\mathrm{Cx} 43$ levels in nasopharyngeal carcinoma cells is also related to promoter methylation (765). The promoter region of Cx32 is methylated and Cx32 expression low in renal carcinomas, where demethylation leads to reexpression of $\mathrm{Cx} 32$, indicating a causal relation $(272,761)$. Reduced Cx26 expression involving DNA methylation has been demonstrated for lung cancer (622), hepatocellular carcinomas (695), and breast cancer $(88,672)$. In contrast, a study on breast cancer showed methylation of the $C x 26$ gene but no correlation to Cx26 expression (634). This was also found to be the case in esophageal tumors (397). The Cx36 promoter is methylated in colon cancer (56); however, increased methylation of the Cx36 promoter does not seem to have any effect on Cx36 expression (637). In fact, when analyzing both methylation and expression data, methylation of the promoter regions of $\mathrm{Cx} 36, \mathrm{Cx} 30, \mathrm{Cx} 37$, and $\mathrm{Cx} 45$ was only found to decrease the expression of Cx45 (637). This study indicates, that although promoter methylation occurs, it does not necessarily translate into corresponding alterations in expression. Therefore, it can be concluded that DNA methylation may be involved in both physiological and pathophysiological changes in connexin expression, but care must be taken when considering determination of methylation in the absence of expression data.

Micro-RNA: The stability and translation of mRNA can be regulated by micro-RNAs and some of these regulate the expression of connexins. Micro-RNAs act by binding to the 3'UTR of the target mRNA, and genomic analysis of the $C x 36, C x 43$, and $C x 45$ genes indicate that multiple binding sites exist (544). A clear physiological function of microRNA-mediated regulation has only been established for $\mathrm{Cx} 43$. During skeletal muscle development, myoblasts fuse to form muscle fibers and during this process expression of Cx43 is lost. During differentiation the micro-RNAs miR1 and miR-206 are upregulated and both reduce expression of $\mathrm{Cx} 43(6,311)$. The reverse is observed in osteoblasts, where differentiation is dependent on Cx43 expression. miR-206 expression in osteoblasts reduces Cx43 levels and inhibits differentiation, which can be prevented by reexpression of $\mathrm{Cx} 43$ even in the presence of miR-206 (286). In contrast to skeletal muscle, high expression levels of $\mathrm{Cx} 43$ are needed for normal conduction in the heart. Patients with coronary artery disease often have heterogeneous and reduced levels of Cx43 and they are at risk of arrhythmia. A study shows that miR-1 is upregulated in these patients, which possibly reduces Cx43 expression. When miR-1 is overexpressed in normal and infarcted rat hearts, conduction is slowed and arrhythmia susceptibility increased. These effects correlate with Cx43 repression and elimination of miR-1 reverses the effects (760). Subsequently, it was shown that miR-1 upregulation and its downstream effects are reversed by the $\beta$-adrenergic agonist propranolol. This suggests that sympathetic stimulation may regulate this pathway (400). The effects of micro-RNAs are also regulated by binding of RNA-binding proteins and in 
the case of miR-1 and miR-206, it was shown that binding of Dnd1 prevented repression of Cx43 (306).

\section{Connexin turnover and trafficking}

Translation and ER sorting-Connexins are believed to be cotranslationally inserted into the ER membrane; however, the experimental evidence for this assumption is limited. It has been reported that $\mathrm{Cx} 26$ and $\mathrm{Cx} 32$ are cotranslated into ER microsomes in vitro and that somewhat surprisingly $\mathrm{Cx} 26$, but not $\mathrm{Cx} 32$, can be posttranslationally incorporated into ER microsomes in their native orientation (778). The extent to which this occurs in vivo is unknown and remains an unexplored possibility. In the ER, folding into mature connexins occurs and intramolecular disulfide bonds are formed between the extracellular loops (294, $541)$.

Once translated, connexins have a very high turnover rate in most cases, with half-lives of a few hours in both cultured cells and native tissues $(30,177,353,691)$. In some cases such as chicken lens cultures, $\mathrm{Cx} 45.6$ and $\mathrm{Cx} 56$ (human orthologs: $\mathrm{Cx} 50$ and $\mathrm{Cx} 46$, respectively) turnover is much lower, around 1.5 days (291), showing that rates differ between connexins and probably also between cell types.

In certain cells with high turnover, the connexins are partly turned over by ER-associated degradation (ERAD) (460), which is responsible for removing immature, damaged, and/or misfolded proteins by retrotranslocation to the cytoplasm, polyubiquitination, and degradation by the proteasome. Monitoring of protein folding is achieved in part by chaperones; however, for connexins it has also been reported that proteolytic cleavage of the NT of misfolded connexins, can target them for degradation after overexpression or prevention of disulfide bond formation $(175,176,713)$. ERAD is often initiated by recognition proteins and Lau and co-workers reported that Cx43 interacts with CIP75 (a ubiquitin-like and ubiquitin-associated domain containing protein) in the ER and that CIP75 expression determines the rate of $\mathrm{Cx} 43$ degradation independent of ubiquitination (378, 666). Although it is normally assumed that ERAD removes damaged/misfolded and thus dysfunctional proteins, this may not hold true for connexins, where ERAD inhibition increases both the number and functional coupling of gap junctions (713). Therefore, regulation of ERAD may be a physiological way of adjusting gap junctional communication. For example, mild heat shock and oxidative stress reduces ERAD of connexins (713), which may represent a protective mechanism whereby cells under stressful conditions can receive, for example, reducing agents such as glutathione from neighboring healthy cells (460).

The Golgi complex and oligomerization-From the ER Cx26 and $\mathrm{Cx} 43$ are transported by COPIImediated transport to the Golgi and depend on the small GTPase Sar1 $(411,681)$. En route to the plasmamembrane, connexins are transported through the Golgi complex, where their presence has been demonstrated $(259,463,532,540,625)$. One exception may be Cx26, which has been proposed to bypass the Golgi (208) although this findingwas later challenged (681). Unlike most membrane proteins, connexins do not oligomerize in the ER but do so after transport into the trans-Golgi network. Connexins are found as monomers when they are prevented from exiting ER $(328,463)$ and fractionation studies show that Cx26 and Cx32 hexamers are exclusively present in the Golgi of liver tissue (145). Multimers have been demonstrated in ER but probably represent aberrant oligomerization when protective mechanisms become saturated. Along the same lines, low expression levels of $\mathrm{Cx} 43$ linked to an ER retention signal produces only monomers, whereas high expression results in substantial oligomerization (591). This feature is shared by several other connexins, where high expression also promotes oligomerization of $\mathrm{Cx} 26$, Cx32, and Cx43 in the ER (714). 
When oligomerization occurs in the ER after overexpression of connexins, gap junction like plaques form between folds of the ER (337), which is probably an unwanted result and a possible reason that mechanisms to prevent oligomerization in the ER have evolved. The exact nature of these mechanisms is unknown but Koval and co-workers showed that ER oligomerization of $\mathrm{Cx} 43$ is prevented by the chaperone ERp29 (123). Possibly, such a mechanism may also exist for other connexins and chaperones.

Membrane insertion and gap junction plaque formation-Trafficking of connexins from the Golgi to the plasma membrane depends on an intact cytoskeleton. For Cx43, the budding of secretory vesicles from the Golgi requires the action of the small GTPase Arf1 (411), and the vesicles are subsequently transported to the plasma membrane. Most studies show that $\mathrm{Cx} 43$ transport depends on microtubules $(213,238,360,680)$, whereas transport of Cx26 (680), Cx30 (537), and Cx31 (257) do not. Cx43-GFP has been demonstrated to traffic via microtubules from the Golgi to the plasma membrane, where connexons diffuse freely and are inserted at the edges of existing gap junction plaques (360). Using an elegant approach of $\mathrm{Cx} 43$ pulse labeling, Gaietta et al. showed that new $\mathrm{Cx} 43$ was added at plaque edges, surrounding older Cx43 in the center (193). Once in the plaque, the movement of Cx43 is highly restricted without mixing of new and old Cx43 $(193,360)$, but this feature is probably not universal to all connexins since the fluorescence of bleached centers in Cx26 plaques were rapidly repopulated by diffusion of $\mathrm{Cx} 26$ from the unbleached plaque perimeter (681). However, the concept of free diffusion and recruitment to the outer perimeter of existing plaques has been challenged as a universal mechanism. Shaw and coworkers showed that transport of $\mathrm{Cx} 43$ via microtubules depends on the microtubule plus end tracking protein EB1, which connects microtubules to adherens junctions and selectively guides $\mathrm{Cx} 43$ to these sites. The study also showed that repopulation of plaques after photobleaching occurred only at the plaque sites and not in neighboring areas, indicating a more direct insertion in the plaque area (617). Such transport mechanisms may be particularly important in tissues with highly organized gap junction structure such as cardiomyocytes, and a subsequent study showed that EB1 is displaced from adherens junctions in stressed myocardium, resulting in reduced insertion of Cx43 at the intercalated discs (639).

Incorporation into plaques renders $\mathrm{Cx} 43$ insoluble in the mild detergent Triton X-100, an event that coincides with the phosphorylation into the P2 isoform (462). Both phosphorylation and the acquisition of Triton insolubility is considered a hallmark of communication competent gap junctions and although the exact overall phosphorylation pattern of the P2 form may vary, it is clear that Cx43 phosphorylated at $S 325 / 328 / 330$ or S328/330 migrate as the $\mathrm{P} 2$ form (355) (for further information on connexin phosphorylation, please see the section on posttranslational regulation).

Internalization-Double membrane gap junction vesicles (termed annular gap junctions) were early on demonstrated by electron microscopy $(217,359)$. These annular gap junctions were later demonstrated to arise when one cell internalizes not only its own connexins but also those from a neighboring cell to which they are docked $(296,468)$. It has been suggested that annular gap junctions arise when whole plaques or large parts thereof are internalized, but for $\mathrm{Cx} 26$ and $\mathrm{Cx} 32$, it was shown that plaques are also internalized as smaller aggregates (191).

In 1979, Larsen et al. reported that annular gap junctions are associated with clathrin (359). Indeed Cx43 is internalized by a clathrin-dependent mechanism $(283,468,520)$ with clathrin being exclusively located at the cytoplasmic surface of the junction (474). Furthermore, inhibition of clathrinmediated endocytosis prevents internalization in response to epidermal growth factor (EGF) (367) and the carcinogen DDT (180). Some components 
of the machinery were later identified, among these were the adaptor proteins Dab2 and AP-2, and the GTPase dynamin $(237,520)$. Dynamin is responsible for the scission of vesicles from the plasma membrane, and dynamin complexes with $\mathrm{Cx} 43$ and the nonreceptor tyrosine kinase c-Src. Dynamin is subsequently activated by c-Src, which induces internalization of Cx43 (215). Clathrindependent endocytosis is also regulated by the small GTPase Arf6 whilst expression of a GTP restricted Arf6 mutant promotes internalization of Cx43 (411), supporting the importance of clathrin-mediated mechanisms.

Calveolin-mediated endocytosis has been implied by the colocalization of calveolins with connexins $(358,380,599)$; however, data suggest that the role of calveolin may be to guide connexins from the Golgi to the plasmamembrane or to stabilize them at the membrane, since calveolin knockdown inhibits intercellular communication (358).

Given the short half-life of connexins, internalization appears to occur continuously, and we know now of several stimuli and physiological processes, which regulate this internalization. Most of these also stimulate degradation and will be discussed later.

Degradation and recycling-Connexin degradation depends on the action of both the lysosomes and the proteasome Targeting for internalization and lysosomal degradation is obtained by monoubiquitination, whereas polyubiquitination targets for proteasomal degradation (317). Proteasomal involvement in connexin breakdown was first described by Laing et al., who showed that a proteasomal inhibitor increased the Cx43 content 6-fold (348). Since then, it has been demonstrated that $\mathrm{Cx} 43$ is subject to ubiquitination, endocytosis, and degradation in response to EGF (367), phorbol esters (368), TNF-a (312), and Gaqcoupled receptors (442). Also, in lens epithelial cells where Cx43 and Cx46 are reciprocally expressed, the expression of $\mathrm{Cx} 46$ downregulates $\mathrm{Cx} 43$ by ubiquitination and subsequent degradation of Cx43 (19).

The proteasome only degrades unfolded proteins and since connexins are internalized as annular gap junctions, the role of the proteasome has been unclear. Combinations of lysosomal and proteasomal inhibitors suggest that degradation is sensitive to both in an nonadditive manner, but there is little evidence to support a sequential mode of action (460).

Several studies show that proteasomal inhibitors prevent the internalization of $\mathrm{Cx} 43$ $(348,350,535)$. This finding led to the proposal that proteasomes regulate endocytosis of Cx43 independent of its actual degradation. One possible mechanism is that inhibition of the proteasome prevents deubiquitination of its substrates and thus leads to a depletion of free ubiquitin. This hypothesis is supported by thework of Rivedal and co-workers, who showed that proteasomal inhibition prevents ubiquitination of $\mathrm{Cx} 43$ (368) and depletes free ubiquitin (366). Likewise, proteasomal inhibition reduces free ubiquitin and prevents ubiquitination of Cx43 after stimulation of Gaq-coupled receptors in rat neonatal cardiomyocytes (442).

In the final step of ubiquitination, ubiquitin is transferred to its target by E3 ubiquitin ligases, many of which recognize PY-motifs in their targets. Such a motif is found in Cx43 and its mutation increased the amount of $\mathrm{Cx} 43$ by decreasing its degradation (678). Subsequently, it was shown that the ubiquitin ligase Nedd4 binds the C-terminus of Cx43 and although knockdown of Nedd4 in rat liver epithelial cells did not alter the total amount of Cx43, membrane localization of Cx43 was increased (372). Since then interaction with Nedd4 has been verified in both Cos-cells (218), corneal fibroblasts (312), and in cardiomyocytes (442). Furthermore, Cx43 was found to be monoubiquitinated (368) suggesting that ubiquitin is essential in targeting $\mathrm{Cx} 43$ to lysosomal degradation. The role of ubiquitination is summarized in Figure 9. 
Annular gap junctions are detected in lysosomes by EM (217). Consistent with the importance of lysosomal breakdown, several studies show that inhibition of lysosomal activity increases the level of Cx43 protein (30, 348, 349, 350, 442, 464, 535, 678). In a number of cell types, this is associated with the accumulation of connexin positive intracellular vesicles $(464,535,678)$. In contrast, lysosomal inhibition in cardiac cells slows the rate of $\mathrm{Cx} 43$ degradation but increases the amount of $\mathrm{Cx} 43$ in gap junction plaques $(30,349)$ and prevents uncoupling after stimulation of Gaq-coupled receptors (442) (442).

Gap junctions may also be degraded by autophagy, and annular gap junctions sequestrated by double membrane structures have been reported (516). Such structures are found in cardiac cells, where some Cx43 colocalizes with the autophagosomal marker LC3. The association of both Cx43 and LC3 with lipid rafts is increased in heart failure, suggesting that autophagy plays a role in degrading $\mathrm{Cx} 43$ in this condition (268). Autophagy also degrades Cx43 and Cx50 in normal rat kidney (NRK) cells during starvation. Intracellular connexins were found to be enclosed in a structure containing LC3 and colocalized with p62, a protein that targets ubiquitinated proteins for autophagy. Furthermore, knockdown of Atg5, which is required for autophagy, prevented starvation induced connexin degradation (379).

Instead of being degraded, some internalized connexin may be recycled back to the plasmamembrane, as suggested by the colocalization of internalized Cx43 with rab4 and rab11, which are markers of recycling endosomes (215). This phenomenon may be particularly important during mitosis, where $\mathrm{Cx} 43$ is endocytosed and recycled to the plasmamembrane in the final stages of mitosis (48).

\section{Posttranslational Regulation of Connexins}

Chemical modification of connexins after their translation is an important tool for the regulation of gap junction channels in all types of tissue. Gap junctions are known to be highly regulated by changes in connexin phosphorylation and ubiquitination, but acetylation, hydroxylation, glutamate $\mathbf{\gamma}$-carboxylation, methylation, palmitoylation, oxidation, deamidation, $S$-nitrosylation, and truncation have also been described $(393,394,552,736)$. Some posttranslational modifications are involved in the gating of electrical and metabolic coupling of mature gap junction channels, whilst others are involved in regulation of the connexin "lifecycle" such as trafficking, assembling, disassembling, and degradation. With the exception of ubiquitination (which was covered in the section on connexin turnover and trafficking), the following section will give an overview of the current knowledge of posttranslational modification of the different connexin family members, how these modifications are mediated, and what the functional consequences are.

\section{Phosphorylation of connexins}

The most extensively studied posttranslational modification of connexins is undoubtedly phopshorylation. That connexins are phosphoproteins was first discovered in the mid 1980s $(578,669)$. Since then a variety of phosphorylation sites have been identified. Classically, connexin phosphorylation is analyzed by studying their electrophoretic mobility, by direct incorporation of $\mathrm{P}^{32}$-phosphate, or by the use of phosphospecific antibodies. When analyzed by SDS-PAGE most connexins display multiple electrophoretic isoforms. For Cx43, this includes a fast migrating "nonphosphorylated" (P0 or NP) band (P0 is not necessarily completely dephosphorylated) and at least two slower migrating bands, usually called P1 and P2 (461). Following alkaline phosphoatase treatment both P1 and P2 end up comigrating with P0 (461). This indicates that phosphorylation is the main posttranslational modification, which determines electrophoretic mobility of $\mathrm{Cx} 43$. Incorporation of radiolabeled phosphate into protein species is one of the oldest biochemical methods for 
phosphorylation analysis and it is also used to study connexin phosphorylation. However, this technique is mostly used for in vitro studies. Finally, phosphor-specific antibodies are also useful to study connexin phosphorylation. With this technique, it is important to note that $\mathrm{p}$-Ser and p-Thr antibodies tend to cross react with other negative charges, which may produce a high number of false positives. Also, the high number and proximity of phosphorylation sites, which are known for most connexins, makes it difficult to develop highly site-specific phosphoantibodies for the use in gap junction phosphorylation analysis.

More recently, mass spectrometry (MS) has revolutionized the area of phospho-proteomics, including the phosphorylation analysis of connexins. This technique enables sitespecific phosphorylation analysis of the entire protein and is able to identify and sequence phosphopeptides even at picomolar level. Furthermore, tandem MS is capable of assigning phosphorylation sites in multiphosphorylated proteins such as connexins, to their specific amino acids.

Using the different available techniques, posttranslational regulation by phosphorylation has been described for all the known connexin family members. With the exception of Cx26, all known connexins contain phosphorylation sites located in their CT tail. In addition, Cx26 (393) as well as Cx36 (700), Cx50 (736), and Cx56 (40) contain phosphorylation sites in the CL, and both Cx26 and Cx32 are reported to be phosphorylated in the NT tail when expressed in HeLa cells, but not when purified from mouse liver (394). Furthermore, several phosphorylation sites are indicated in the extracellular domain E2 of Cx26 (393). It should be noted that not all connexins have been subject to systematic MS analysis of phosphorylation sites. Therefore, it is reasonable to assume that additional phosphorylation sites may be identified in the future.

Phosphorylation events are essential for the correct oligomerization of connexins to connexons and also for the correct composition and function of complete dodecameric gap junction channels. The intracellular CT domain, which contains the majority of identified phosphorylation sites, is highly variable amongst different connexin family members. Therefore, phosphorylation of the CT tail influences the function of gap junction channels in a connexin-specific manner.

Phosphorylation of Connexin43-Cx43 is the most abundant and ubiquitously expressed connexin in mammalian cells and also the most extensively studied connexin. The immature Cx43 protein is initially translated as a $40 \mathrm{kDa}$ protein chain, which is phosphorylated soon after synthesis. The phosphorylation status of the protein then changes as it traffics through the endoplasmatic reticulum and Golgi to the plasma membrane, ultimately forming a gap junction channel. (For further information on connexin synthesis and trafficking, see previous section). To date, 21 putative phosphorylation sites have been identified in the CT domain of Cx43 (Table 3).

As seen in Table 3, the phosphorylation sites in $\mathrm{Cx} 43$ are primarily serine amino acids (for references see Table 3); however, phosphorylation of Tyr247 and Tyr265 (93, 356, 357, $383,645,687)$, as well as threonine phosphorylation (122) has also been reported for $\mathrm{Cx} 43$. Some of the specific phosphorylation sites in $\mathrm{Cx} 43$ are implicated in the regulation of electrical and metabolic coupling of mature gap junction channels; whereas other sites seem to regulate the various stages of the connexin "life cycle" including hemichannel oligomerization, export of the protein to the plasma membrane, gap junction assembly, and connexin degradation $(356,357)$.

Moreno and colleagues were the first to show that gap junction channels composed of Cx43 exhibit distinct unitary conductance values, which are correlated with the phosphorylation 
state of the protein $(450,452)$. Shortly after, Kwak et al. found that activation of protein kinase $\mathrm{G}$ (PKG) by 8-bromoguanosine $3^{\prime}: 5^{\prime}$-cyclicmonophosphate (8Br-cGMP) increased incorporation of $\mathrm{P}^{32}$ into rat $\mathrm{Cx} 43$ (but not human $\mathrm{Cx} 43$ ) expressed in SKHep1 cells, which was associated with a decreased gap junctional conductance (344). Most likely, PKG phosphorylates Ser257 of rat $\mathrm{Cx} 43$. In the human $\mathrm{Cx} 43$ sequence, amino acid residue 257 is replaced by an alanine, which cannot be phosphorylated. Later on, dephosphorylation of Ser368 in Cx43 was shown to occur with a time course similar to that of electrical uncoupling of cardiomyocytes in the ischemic heart, with no concomitant changes in the total amount of $\mathrm{Cx} 43$ (31). Finally, a MS study conducted on $\mathrm{Cx} 43$ purified from Langendorff perfused rat hearts exposed to different time frames of ischemia showed various time dependent changes in $\mathrm{Cx} 43$ phosphorylation (14). Specifically, this study showed that Ser306 become fully dephosphorylated within the first $7 \mathrm{~min}$ of ischemia, whereas Ser330 become phosphorylated. Between 15 and $30 \mathrm{~min}$ of ischemia, the critical time interval where gap junction uncoupling occurs, Ser297 and Ser368 also become fully dephosphorylated. In another study, ischemic preconditioning of isolated rat hearts was found to cause a $34 \%$ decrease in the maximal rate of uncoupling together with a diminished overall dephosphorylation of $\mathrm{Cx} 43$ during a subsequent period of prolonged ischemia (287). Based on this observation, the authors concluded that preservation of phosphorylated $\mathrm{Cx} 43$ in gap junctions is responsible for delayed uncoupling induced by preconditioning. Taken together, these findings shows that site specific phosphorylation of $\mathrm{Cx} 43$ is a key mechanism for the regulation of electrical coupling through gap junction channels composed of $\mathrm{Cx} 43$. Furthermore, the gating of $\mathrm{Cx} 43$ channels seem to be based on an interplay between various phosphorylation sites.

To further address the functional consequences of the site specific phosphorylations involved in gap junction uncoupling during ischemia, Procida and co-workers (530) conducted a study examining the effect of serine to alanine substitutions of position 296, 297, and 306 of Cx43. (An alanine substitution mimics a constitutively dephosphorylated form of a serine residue.) Alanine substitution of Ser296 or Ser297 in Cx43 had no significant effect on either macroscopic electrical coupling or single-channel conductance in HeLa cells. However, an alanine substitution of Ser306 was found to reduce electrical coupling to 57\% (530). The reduced coupling occurred in the absence of changes in the total number of gap junction channels. Instead the change in electrical coupling was mediated by a reduced single-channel conductance (530). Based on these data, it seems reasonable to conclude that Ser306 is an important regulatory phosphorylation site, which is involved in electrical uncoupling during cardiac ischemia.

When Ser306 was first discovered as a phosphorylation site, $\mathrm{Ca}^{2+} /$ calmodulin-dependent protein kinase II (CaMKII) and other kinases were tested for their ability to phosphorylate small synthetic peptides containing this specific phosphorylation site. However, the responsible kinase was not identified. In contrast, a recent study using a peptide containing the full length of the CT tail of $\mathrm{Cx} 43$, showed that Ser306 is a target for in vitro phosphorylation by CaMKII (282). CaMKII is known to play a central role in the regulation of a variety of cellular functions in the heart such as $\mathrm{Ca}^{2+}$ homeostasis, transcription, and apoptosis $(61,785)$. In addition, increased expression and activation of CaMKII occurs in cardiac disease states such as hypertrophy, heart failure, myocardial ischemia, and infarction $(410,769)$. However, the specific role of CaMKII in the regulation of Ser306 during ischemia remains to be further investigated.

Another specific phosphorylation site of $\mathrm{Cx} 43$, which seems to be highly involved in the gating of mature gap junction channels, is Ser368. As already mentioned, Ser368 becomes dephosphorylated during cardiac ischemia with a time course similar to that of electrical uncoupling $(14,31)$. Studies have also shown that 12-O-tetradecanoyl-phorbol 13-acetate 
(TPA)-induced protein kinase $\mathrm{C}$ (PKC) phosphorylation of $\mathrm{Cx} 43$ is associated with reduced single-channel conductance $(346,354)$, which can be prevented by substitution of Ser368 to an alanine (354). However, it should be noted that PKC may not only phosphorylate Ser368 but also Ser365, Ser369, Ser372, and Ser373 of Cx43 (616). Despite the reduction in singlechannel conductance following TPA-induced Cx43 phosphorylation, Kwak and co-workers found that the macroscopic electrical coupling was increased (346). This indicates that PKCinduced phosphorylation influences the open probability of $\mathrm{Cx} 43$ gap junction channels.

The effect of Ser368 phosphorylation on dye coupling has been examined in cardiomyocytes, HeLa cells, and fibroblasts, but with contradicting results. In HeLa cells, it was found that mutation of Ser368 to an alanine results in a slight (statically insignificant) decrease in dye coupling compared to wt Cx43 (767). However, when alanine substitutions were introduced into Ser365, Ser368, Ser369, and Ser373 all at once, dye coupling was severely reduced (767). This implies that phosphorylation of Ser368 itself is not crucial for Cx43-mediated dye coupling in HeLa cells, whereas simultaneous dephosphorylation of all of the phosphorylation sites located in the extreme CT region causes a marked drop in dye transfer. Contradictory to these findings, TPA-induced PKC phosphorylation of Cx43 has been shown to contribute to a decrease in dye coupling in both neonatal cardiomyocytes (346) and fibroblasts (354), which is probably mediated by a conformational change of Cx43 (22). Further studies are needed to determine the reason for the different findings on the effect of Ser368 and PKC phosphorylation on dye coupling, but one potential explanation could simply be the differences in cell systems used. All together, these data shows that PKC and the phosphorylation sites (including Ser368) located in the extreme CT region of $\mathrm{Cx} 43$ play a key role in the regulation of both electrical and metabolic coupling of gap junction channels composed of $\mathrm{Cx} 43$.

Compared to $\mathrm{PKC}, \mathrm{Cx} 43$ has been found to be a relative poor substrate for cAMP-dependent protein kinase (PKA). However, PKA is able to phosphorylate both Ser364 and Ser365 in Cx43 and phosphorylation of Ser364 and/or Ser365 seems to accelerate a subsequent PKCinduced phosphorylation of other serines in the 362 to 376 region (616). In support of this, H89 (a PKA inhibitor) was found to inhibit follicle stimulating hormone (FSH)-induced phosphorylation of Ser365, Ser368, Ser369, and Ser373 of Cx43 in granulosa cells (768). However, another study found that Ser365 phosphorylation or a mimicked phosphorylation (mutation of Ser365 to an Asp) reduces PKC-induced phosphorylation of Ser368 during ischemia (646). Though contradictory, these findings illustrate that different phosphorylation sites may interact with each other and add to the complexity of $\mathrm{Cx} 43$ phosphorylation.

Tyrosine phosphorylation is also a subject for contradicting results regarding its role in the regulation of Cx43 gap junction channels. Src kinase-mediated phosphorylation of Tyr265 has been found to reduce electric conductance between fibroblasts without affecting the expression of mature gap junction channels $(212,527)$. On the other hand, Tyr265 has also been implicated in the regulation of an interaction between $\mathrm{Cx} 43$ and the cytoskeleton protein zonula occludens-1 ( $\mathrm{ZO}-1$ ), which is important for the localization of $\mathrm{Cx} 43$ at the intercalated discs of cardiomyocytes (687). Thereby, in contrast to the findings by Giepmans and co-workers, phosphorylation of Tyr265 would lead to a reduction in the amount of Cx43 present at the cell surface.

In addition to phosphorylating tyrosine residues in $\mathrm{Cx} 43$, Src kinase may also induce mitogen-activated protein kinase (MAPK) to phosphorylate Cx43. MAPK is able to phosphorylate Ser255, Ser279, and Ser282 of Cx43 (739). Phosphorylation of these MAPK sites is not required for normal gap junction assembly in HeLa cells, but phosphorylation of Ser279 and/or Ser282 is correlated with a decrease in both dye coupling and electrical conductance (738). Inhibition of dye coupling by EGF has also been linked to direct 
phosphorylation of Cx43 by MAPK (565). Studies have indicated that TPA-induced uncoupling of $\mathrm{Cx} 43$ gap junction channels is not only mediated by PKC phosphorylation, but rather by interplay between PKC and the MAPK pathway $(565,638)$.

Ser325, Ser328, and/or Ser330 are closely involved in the trafficking of Cx43. Cooper and Lampe found that casein kinase 1 (CK1)-induced phosphorylation of Ser325, Ser328, or Ser330 regulate $\mathrm{Cx} 43$ gap junction assembly in normal rat kidney cells (109). Also, using an antibody specific for phosphorylation at Ser325, Ser328, and Ser330, it was shown that these sites are phosphorylated in the gap junction plaqueassociated isoform of $\mathrm{Cx} 43$ (643). Furthermore, during cardiac ischemia, relocation of $\mathrm{Cx} 43$ from the intercalated disk to the lateral edges of the myocytes, is correlated with dephosphorylation of Ser325, Ser328, and/ or Ser330 (355). Also, mouse fibroblasts expressing Cx43 in which Ser325, Ser328, or Ser330 is mutated to alanine show decreased dye coupling and significantly delayed development of electrical coupling compared to cells expressing wt Cx43 (355). Taken together, these data demonstrate a role for phosphorylation of Ser235, Ser328, and Ser330 in the regulation of efficient gap junction assembly and Cx43 lateralization.

As already indicated, some of the contradicting findings regarding the effect of site-specific Cx43 phosphorylation may be explained by the differences in test systems, that is, different cell types or studies performed on either cell cultures or tissue. A study has shown that most $\mathrm{Cx} 43$ exists in a semiphosphorylated or dephosphorylated form when expressed in $\mathrm{HeLa}$ cells (644), whereas cardiomyocytes have been shown to contain mainly phosphorylated Cx43 (353). Importantly, it must also be noted that electric and metabolic coupling may be conversely affected. Studies by Kwak and co-workers have found that TPA-induced PKC activity in cardiomyocytes leads to increased gap junction conductance accompanied by a fall in dye coupling $(342,346)$. Also, another study evaluating several Cx43 expressing cell lines showed no correlation between electrical and metabolic coupling (164). These observations are important since some studies only measure either electrical conductance or dye transfer and uses the result as a measure for changes in gap junction communication. In addition, the ability to phosphorylate and/or the effects of site-specific changes in $\mathrm{Cx} 43$ phosphorylation may depend on the existing phosphorylation state of $\mathrm{Cx} 43(616,768)$. Finally, is seems that several kinases are able to phosphorylate some of the specific phosphorylation sites located in $\mathrm{Cx} 43$ (Table 3 ). All of this underlines the extremely complex nature of $\mathrm{Cx} 43$ phosphorylation.

Dephosphorylation of Connexin43 by protein phosphatases-Compared to the knowledge we have about protein kinases involved in phosphorylation of connexins, far less is currently known about the protein phosphatases (PPs), which are responsible for dephosphorylation of connexins.

Based on their amino acid sequences and crystal structures, phosphoserine and phosphothreonine PPs are divided into three different gene families: phosphoproteinphosphatases (PPPs), metal-dependent protein phosphatases (PPM), and aspartatebased phosphatases such as FCP [TFIIF-associating component of RNA polymerase II carboxyterminal domain (CTD) phosphatase] and small CTD phosphatase (SCP) (619). PPPs are the largest class of PPs and they are further divided into the subgroups PP type 1, $2 \mathrm{~A}, 2 \mathrm{~B}, 4,5,6$, and 7 based on their sequence $(101,619)$.

Currently, PP1, PP2A, and PP2B have been connected to dephosphorylation of Cx43 (267). Chronic activation of $\mathrm{PP} 2 \mathrm{~B}$ is associated with decreased expression levels and redistribution of $\mathrm{Cx} 43$ in mouse hearts. Furthermore, the present $\mathrm{Cx} 43$ is found mainly in its dephopshorylated form (91). Okadaic acid and calyculin A (potent inhibitors of PP1 and PP2A) both delay electrical uncoupling of gap junctions during ATPdeprived conditions in 
neonatal cardiomyocytes (158). However, an even better preservation of conductance was obtained in the presence of the selective PP1 inhibitors heparin and PP inhibitor 2 (I2) (158). Furthermore, in the presence of ATP, addition of $p$-nitrophenyl phosphate (a specific PP1 stimulator) led to gradual gap junction uncoupling, unless I2 was added simultaneously (158). Together, data clearly indicate a role for PP1 in the regulation of gap junctions during ATP depletion. This conclusion is further supported by findings in isolated perfused rat hearts and adult cardiomyocytes, where the presence of okadaic acid and calyculin A, but not fostriecin (a selective PP2A inhibitor) was found to decrease $\mathrm{Cx} 43$ dephosphorylation during simulated ischemia (290). In contrast to these findings, it was previously shown that fostriecin mimics the effect of ischemic preconditioning in ischemic adult cardiomyocytes from both pigs and rabbits (12). Furthermore, inhibition of PP2A was also found to reduce infarct size following myocardial infarction in isolated rabbit hearts, even when administrated after the onset of ischemia (740). This indicates a role for $\mathrm{Cx} 43$ dephosphorylation in infarct development. The authors further found that the effect of PP2A inhibition on infarct size was similar to that of ischemic preconditioning, even though ischemic preconditioning had no effect on the activities of either PP1 or PP2A (740).

At normal physiological conditions, both PP1 and PP2A have been found to colocalize with $\mathrm{Cx} 43$ in the left ventricle of rabbit hearts (3). Ai and Pogwizd further found that heart failure induced by aortic constriction caused an increase in the proportion of nonphosphorylated Cx43, which was accompanied by an increased level (over 2.5-fold) of colocalized PP2A, whereas colocalized PP1 remained unchanged (3). PP1 and PP2A are also present in the left ventricle of minipigs; however, only PP2A was found to colocalize with $\mathrm{Cx} 43$ in this species (686). When isolated hearts were exposed to 90 min of low flow ischemia, it induced dephosphorylation of $\mathrm{Cx} 43$ along with an increase in total PP2A levels. However, the amount of PP2A, which coprecipitated with $\mathrm{Cx} 43$, was not affected during ischemia. Furthermore, Totzeck et al. found that although ischemic preconditioning preserved $\mathrm{Cx} 43$ phosphorylation during ischemia, ischemic precondition did not affect PP2A levels or activity (686). Since the lack of effect of ischemic preconditioning on PP2A as well as PP1 level and activity was also shown for rat hearts (740), it seems that preservation of Cx43 phosphorylation by ischemic preconditioning may not be directly mediated by changes in phosphatase activity. However, the presented data leave no doubt that PPs are involved in the regulation of $\mathrm{Cx} 43$ phosphorylation during different pathophysiological conditions.

Posttranslational modifications of Cx26 and Cx32-Posttranslational modifications of Cx26 and Cx32 (the major liver gap junction proteins) immunopurified from HeLa cells and mouse liver have been examined by matrix-assisted laser desorption/ionization time-offlight MS (MALDI-TOF-MS) (394). This study was the first to identify posttranslational modifications of $\mathrm{Cx} 26$, which include hydroxylation and/or phosphorylation in the NT domain, as well as Y-carboxylation in the CL. Importantly, they also found that the postranslational modifications differ depending on whether Cx26 is expressed in liver tissue or HeLa cells. Specifically, the NT of Cx26 was hydroxylated (in position Asp2 or Asn14) when expressed in liver cells, whereas the same peptide fragment was phosphorylated (in position Asp2, Thr5, or Ser8) when expressed in HeLa cells. Furthermore, the CL of Cx26 purified from mouse liver contained $\mathrm{Y}$-carboxylations (on Glu114, Glu119, or Glu120), which was not detected in Cx26 from HeLa cells (394). Later on, acetylation of the NT domain and at several lysine residues of the $\mathrm{CT}$ tail have been identified, along with additional hydroxylation sites in the CL, Y-carboxylation sites in the extracellular domain E1, methylation sites in E1 and the CT domain, as well as several phosphorylation sites in E2 (393). Many of the identified posttranslational modification sites of Cx26 have also been connected to disease-causing mutations. However, the exact regulatory and functional role of the posttranslational modifications of these sites remains to be established. 
The posttranslational modifications identified for $\mathrm{Cx} 32$ from mouse liver are phosphorylation of the NT domain (Thr4, Tyr7, Thr8, or Ser11), Y-carboxylation of the CL (two of Glu109, Glu118, or Glu119), as well as palmitoylation of the CT (either Cys280 or Cys283). Y-carboxylation of the CL was also detected in Cx32 from HeLa cells. However, in contrast to the posttranslational modifications identified in liver cells, phosphorylation or hydroxylation of the NT domain (Asn2 or Asn14) and phosphorylation of the CT tail (His237, Ser233, or Ser240) was identified for Cx32 from HeLa cells (394). In addition to the posttranslational modification sites identified for Cx32 by MS, studies in hepatocytes have shown that Ser233 can be phosphorylated by PKA and PKC (580). Furthermore, CaMKII was also found to phosphorylate unspecified serine and threonine residues, but not Ser233 in Cx32 (580).

As for $\mathrm{Cx} 43, \mathrm{Cx} 32$ has also been identified as a target for EGF receptor tyrosine kinase (146). Itwas shown that calmodulin binds to Cx32 in the absence of calcium, which thereby prevents phosphorylation of $\mathrm{Cx} 32$ by EGF-receptor tyrosine kinase. This led the authors to conclude that the phosphorylation site(s) for EGF-receptor tyrosine kinase is located in a calmodulin-binding site of $\mathrm{Cx} 32$. Both residue 1-27 in the NT domain and residue 216 to 230 of the CT tail of Cx32 have been identified as calmodulin binding sites in Cx32 (685). Since Tyr7 was identified as a potential phosphorylation site in Cx32 purified form both mouse liver and HeLa cells (394), this could, potentially, be the target for EGF-receptor tyrosine kinase. However, this remains to be experimentally verified.

The available data on different posttranslational modifications of Cx26 and Cx32 indicate that in addition to the well-described phosphorylation of connexins, many other posttranslational modifications are also involved in the regulation of gap junction channels. Furthermore, each connexin seem to be differently processed depending on the cellular background, which is important when comparing studies conducted in different cell or tissue systems.

Phosphorylation of Connexin40 and Connexin45-The posttranslational regulation of $\mathrm{Cx} 40$ and $\mathrm{Cx} 45$ has been studied less extensively than other connexins. Activation of both PKA and PKC have been found to increase $\mathrm{P}^{32}$ incorporation in to Cx40 in transfected human cells (689) and administration of 8-Br-cAMP causes a mobility shift of $\mathrm{Cx} 40$ when examined by western blotting (710). When expressed in SKHep1 cells, PKA-induced phosphorylation of $\mathrm{Cx} 40$ results in increased macroscopic gap junction conductance and Lucifer Yellow permeability of Cx40 gap junction channels (710). Cx40 is also phosphorylated in neural layers of the mammalian retina (421) and dephosphorylation of Cx40 was recently found to be a key mechanism in electrical uncoupling between microvascular endothelial cells (ECs) during sepsis (52). Specifically, Bolen and co-workers found that reduced serine phosphorylation of $\mathrm{Cx} 40$ is responsible for reduced electrical coupling following exposure to lipopolysaccharide (LPS) during hypoxia and reoxygenation. Furthermore, they found that this uncoupling could be prevented by PKA activation and mimicked in control cells by PKA inhibition (52).

In addition to the vasculature and retina, $\mathrm{Cx} 40$ is also highly expressed in the atria and conducting system of the myocardium (231). Changes in Cx40 expression and distribution, as well as $C x 40$ gene mutations and polymorphisms all play a role in development and maintenance of atrial fibrillation (AF) (84). Currently, the role of posttranslational modifications of $\mathrm{Cx} 40$ in $\mathrm{AF}$ remains unknown. However, based on the important role phosphorylation of $\mathrm{Cx} 43$ has in ventricular arrhythmias, it seems reasonable to assume that phosphorylation or other posttranslational modifications of $\mathrm{Cx} 40$ may also play a significant role in $\mathrm{AF}$. 
Serine phosphorylation of $\mathrm{Cx} 45$ has been shown in a variety of cell types $(79,122)$. Western blots show that Cx45 migrate as a doublet including a 46 and a $48 \mathrm{kD}$ band, where the 48 $\mathrm{kDa}$ band represents the phosphorylated form (711). The specific phosphorylation sites of $\mathrm{Cx} 45$ remain to be determined, but the relative intensity of the $48 \mathrm{kDa}$ band in a western blot increases upon activation of PKA and MAPK (711). Furthermore, PKC activation has been reported to decrease $\mathrm{Cx} 45$ single-channel conductance (341) and increase the macroscopic conductance (711) of Cx45 gap junction channels. However, the effect on macroscopic conductance occurred in the absence of any visible changes in $\mathrm{Cx} 45$ phosphorylation when examined by western blot (711). In contrast, activation of PKA or MAPK, which increases Cx45 phosphorylation, decreases macroscopic junctional conductance (711). These studies demonstrate that various protein kinases are differentially involved in phosphorylation and regulation of electrical coupling of gap junction channels composed of $\mathrm{Cx} 45$.

\section{Posttranslational modifications of Connexin46 and Connexin50-Cx46 and}

$\mathrm{Cx} 50$ combine to form the gap junctions in ocular lens fiber cells. These gap junctions play an important role in normal lens growth and maintenance of lens transparency $(225,569$, 607) and mutations in the $C x 46$ and $C \times 50$ genes are associated with development of cataracts $(405,661)$. (For further information on the physiological role of Cx46 and Cx50 in the eye, see Section "Physiological Function in Cellto-Cell Communication.")

In addition to posttranslational regulation by phosphorylation, CT cleavage of lens fiber connexins has also been proposed to occur naturally during the maturation of lens fiber cells $(382,766)$. Calpain was identified as the enzyme that removes a $32-\mathrm{kDa}$ portion of the CT tail of bovine Cx50 and the same study identified Glu290 and Ser300 as cleavage sites (382). In contrast, two other studies have identified His284, Phe286, and Glu290 $(618,736)$ as targets for truncation of bovine $\mathrm{Cx} 50$ and yet another study has identified residue Glu365 as a target for caspase-3-like protease induced truncation in chicken $\mathrm{Cx} 45.6$ (the chicken homologue of Cx50) (766). Yin and co-workers further showed that the cleavage of chicken Cx45.6 was inhibited by casein kinase II (CKII)-induced phosphorylation of Ser363 (766), indicating that chicken $\mathrm{Cx} 45.6$ truncation is regulated by phosphorylation. A recent MS study identified a total of 18 and 11 phosphorylation sites in bovine Cx50 and Cx46, respectively (736). For $\mathrm{Cx} 46$, all of the identified phosphorylation sites are located in the CT domain, whereas three of the identified phosphorylation sites in $\mathrm{Cx} 50$ are located in the $\mathrm{CL}$ (736). In addition, five truncation sites, all located in the $\mathrm{CT}$ region, were identified for Cx50, whereas seventeen truncation sites, located in the NT, the CT, and in the CL, were observed in $\mathrm{Cx} 46$. Furthermore, the distribution of truncations at the various sites for both $\mathrm{Cx} 50$ and $\mathrm{Cx} 46$ was highly variable between samples obtained from the nucleus and from the cortex of the lens. Some truncations are specific to either nucleus or cortex samples, whereas other sites are detected in both samples (736). Based on these data, it seems reasonable to conclude that truncation of $\mathrm{Cx} 46$ and $\mathrm{Cx} 50$ is a highly regulated mechanism, which could (at least partly) be controlled through the phosphorylation status of the protein.

The physiological consequences of truncation and phosphorylation status of lens fiber connexins have not been completely elucidated. However, the CT regions of Cx50 and $\mathrm{Cx} 46$, which contains most of the identified phosphorylation and truncation sites, seem to play important roles in channel gating and permeability. Several studies have shown that cleavage of the CT does not abolish the ability to form functional channels $(140,162,756)$. Some studies have shown that truncated lens connexins retain their sensitivity to cytosolic acidification $(140,162,662)$, whereas others have found that cleavage of the CT of Cx50 abolishes $\mathrm{pH}$ gating in vitro (756). However, differences in experimental design may again underlie these conflicting results. The greatest physiological consequence of Cx50 truncation is a significant reduction in macroscopic coupling, which is found to occur without affecting unitary conductance $(140,656)$. However, the specific effect of decreased 
macroscopic conductance in lens fiber cells and how this may contribute to the development of cataracts still remains to be established.

\section{S-nitrosylation of connexins}

$S$-nitrosylation, the covalent attachment of a nitrogen monoxide group to the thiol side chain of cysteine, has emerged as an important mechanism for dynamic, posttranslational regulation of many proteins, in a way comparable to that of phosphorylation. $S$-nitrosylation represents a form of redox modulation, which may occur during oxidative stress, and it is believed to be a common mediator of nitric oxide (NO) effects $(269,419)$. In support of a role of cysteine residue nitrosylation in $\mathrm{Cx} 43$ hemichannel opening, Retamal et al. found that a brief application of dithiothreitol (DTT) to metabolically inhibited astrocytes decreases dye uptake and $S$-nitrosylation of surface $\mathrm{Cx} 43$ without any apparent effect on the degree of dephosphorylation (556). Moreover, they demonstrated that dye uptake induced by NO donors is greatly reduced by DTT and by hemichannel blockers. Notably, NO donors had little effect on the amount of surface $\mathrm{Cx} 43$ or its phosphorylation state (556).

Another study have also emphasized a critical role for $S$-nitrosylation/denitrosylation of $\mathrm{Cx} 43$ in the regulation of communication between smooth muscle cells and ECs in the blood vessel wall (664). They found that $\mathrm{Cx} 43$ is constitutively $S$-nitrosylated on Cys 271 by active endothelial NO synthase compartmentalized at the myoendothelial junction, whereby the permeability of the gap junction channel is enhanced. Conversely, stimulation of smooth muscle cells with the constrictor phenylephrine caused Cx43 to become denitrosylated by $S$ nitrosoglutathione reductase, which thereby attenuated channel permeability (664).

A single study on Cx46 S-nitrosylation has also been conducted. Retamal and co-workers examined the effects of the NO donor $S$-nitrosoglutathione (GSNO) on the electrical properties and fluorescent-dye permeability of $\mathrm{Cx} 46$ hemichannels expressed in Xenopus laevis oocytes (557). GSNO was found to enhance voltage sensitivity, increase tailcurrent amplitude, and change activation and closing kinetics of hemichannels composed of both wt $\mathrm{Cx} 46$ and a $\mathrm{Cx} 46$ mutant, in which the CT domain was replaced with that of $\mathrm{Cx} 43$ (557). However, the effect of GSNO vanished when hemichannels was composed of mutated $\mathrm{Cx} 46$, in which the intracellular and TM helix cysteines were mutated to alanine. Therefore, they concluded that $\mathrm{Cx} 46$ hemichannels are sensitive to NO and that the NO effects are mediated by $S$-nitrosylation of one or more of the intracellular cysteines. However, at the same time they also found that NO had no major effects on $\mathrm{Cx} 46$ hemichannel permeability at normal resting potential (557). Therefore, it seems unlikely that NO induced $S$ nitrosylation of $\mathrm{Cx} 46$ is involved in the development of cataract.

\section{Final considerations}

Although phosphorylation is the most extensively studied form of posttranslational modification of connexins, the recent progress in the quality of MS studies have made it possible to identify other forms of posttranslational modifications. Therefore, it is now clear that although phosphorylation of connexins is very important in the regulation of connexins, phosphorylations may regulate and interact with other forms of posttranslational modifications to regulate generation, gating, and degradation of gap junction channels. The experimental research needed to evaluate the biological effects and relevance of the different forms of chemical modifications have only just begun, and there is still a long way to go before we completely understand the complexity of posttranslational regulation of connexins in health and disease. 


\section{Physiological Function in Cell-to-Cell Communication}

Virtually all cells in solid tissues are joined by gap junctions that allow transport of small molecules such as amino acids, sugars, small peptides, and intracellular messengers between cells, and in addition provides electrical coupling between cells by allowing the transfer of electrical charge carried by various ion species, for example, $\mathrm{K}^{+}, \mathrm{Na}^{+}, \mathrm{Cl}^{-}$, and $\mathrm{Ca}^{2+}(574)$. Gap junctions therefore provide a universal function as intercellular channels of communication that form the basis of direct cellular interactions. Given the relatively low specificity of gap junctions that allows passage of a wide variety of molecules, it is not surprising that they are involved in a wide variety of physiological functions in different cell types. In many cases our knowledge stems from the effects of human mutations in connexin genes and the effects of targeted gene disruptions in mice $(149,630,787)$. In general, the association of genetic and acquired disturbance of connexin function with human diseases have emphasized the importance of proper intercellular communication and shed new light on the physiological role of gap junctions for recent reviews see $(351,787)$. It is interesting that in some cases a specific function is directly related to a specific connexin isoform, whereas elsewhere several isoforms may be substituted for one another without any apparent functional consequences. In addition, there are examples where the function of gap junctions are unrelated to their function as intercellular channels (167) (see also section on noncoupling functions of connexins), emphasizing the great complexity of the field.

\section{Cardiovascular system}

Heart-Gap junctions are present in most cells of the cardiovascular system. The heart expresses several connexin isoforms with $\mathrm{Cx} 43, \mathrm{Cx} 40$, and $\mathrm{Cx} 45$ being the dominant isoforms $(127,128,335)$. Gap junctions play a critical role by allowing the passage of current between the cells of the myocardium, which is a requirement for the propagation of the action potential across the heart. In working myocardium, gap junctions are preferentially present in the region of the intercalated disks providing an "end-to-end" coupling of the cardiomyocytes $(581,610)$. The uneven distribution of cardiac gap junctions in atrial and ventricular cardiomycytes partly determines the anisotropy of cardiac tissue, with the relative paucity of "side-to-side" gap junctions leading to preferential conduction of the action potential along the long axis of the cardiomyocytes (581). The connexins have different distributions in the specialized cardiac tissues. $\mathrm{Cx} 43$ is the major connexin in the working myocardium of the ventricles $(581,610,722)$, whereas the atrial cardiomyocytes express a mixture of $\mathrm{Cx} 40, \mathrm{Cx} 43$, and $\mathrm{Cx} 45(128,231,706)$. Mice that are heterozygous for the $\mathrm{Cx} 43$ null mutation $\left(\mathrm{Cx} 43^{+/-}\right)$are reported to have either slowed epicardial conduction in the ventricles, and a widening of the QRS complex $(235,679)$, or to have no apparent changes in conduction $(455,708)$. Apparently, reductions larger than $50 \%$ are needed to get robust effects on conduction $(121,708)$, but during ischemia $\mathrm{Cx} 43^{+/-}$mice are more prone to arrhythmias (369). The specialized structure of the conduction system, that is, the sinoatrial- and AV-nodes, and the Hiss-Purkinje system, has been found to express $\mathrm{Cx} 40$, whereas $\mathrm{Cx} 43$ are expressed to a lesser extent $(229,347,486,487,581,610,707)$. In agreement with this, $\mathrm{Cx} 40$ null mice have cardiac conduction abnormalities like atrioventricular and bundle branch block $(42,632)$. $\mathrm{Cx} 45$ and $\mathrm{Cx} 31.9(\mathrm{mCx} 30.2)$ are expressed in the AV node and in the center of the sinoatrial node (58). Recent data from mice indicates that $\mathrm{Cx} 31.9$ (mCx30.2), which has the lowest unitary conductance among the connexins, may play a significant role in the slow propagation of the action potential through the AV node $(334,335,598)$. The relevance of these findings for humans remains controversial as a recent study has failed to find any expression of $\mathrm{Cx} 31.9$, which is the human ortholog of mouse $\mathrm{Cx} 30.2$, in the conduction system (333). 
Cx43 also plays a role in cardiac development. Mice that lack Cx43 survive to birth and are born with beating hearts. However, they die shortly after birth due to obstruction of the right ventricular outflow tract, an abnormality that prevents normal perfusion of the lungs (549). In humans, there also appears to be a link between mutations in $\mathrm{Cx} 43$ and cardiac malformations in that mutations has been associated with the hypoplastic left heart syndrome and atrioventral canal defects in pediatric patients (124).

Alterations in both the amount and cellular distribution of gap junctions have been reported in many types of cardiac disease, and it has been suggested that these changes may cause arrhythmias and/or sudden cardiac death $(156,221,611,612,613,614,615)$. Cardiac diseases are often associated with a reduced and/or heterogeneous expression of connexins (288). As a result, there will be slowing of conduction and enhancement of discontinuities in cardiac anisotropy, both of which is believed to predispose to reentrant arrhythmias (322). It also appears as if chronic arrhythmias by themselves result in electrical remodeling of the heart with an altered expression and distribution of gap junctions (613). Attempts to design drugs that enhance gap junction conductance have shown some promise in treating and/or preventing reentrant arrhythmias $(13,245,261,318,319,320,538,659,755)$ (see section on pharmacology for further information).

Besides current, cardiomyocytes may also exchange biologically important molecules through gap junctions. This may have significance with regard to the progression and spread of cell injury and death during myocardial ischemia. Nonselective pharmacological uncoupling of gap junctions has been found to reduce infarct size following acute ischemia $(196,583)$, and mice heterozygous for the $\mathrm{Cx} 43$ null mutation $\left(\mathrm{Cx} 43^{+/-}\right)$developed smaller infarcts compared to wt mice following coronary artery ligation (302). The issue remains controversial, since studies using enhancers of gap junction activity also reported reduced infarct size following chronic infarction (252) and acute ischemia reperfusion (262). The discrepancies may be the result of the use of relatively unspecific pharmacologic agents, different species of experimental animals, and different experimental protocols in the studies.

Blood vessels-In the vascular system, gap junctions couple the vascular smooth muscle cells (VSMC), the ECs, and also connect ECs to underlying smooth muscle cells via myoendothelial gap junctions (244). The major connexins found in blood vessels are Cx37, $\mathrm{Cx} 40, \mathrm{Cx} 43$, and $\mathrm{Cx} 45$. The intercellular communication in the vasculature contributes to the regulation of resistance in different vascular beds according to metabolic needs, and therefore ultimately participates in the regulation of blood pressure. Pathological conditions, such as atherosclerosis, diabetes, and hypertension are associated with changes in connexin regulation and expression $(46,243,246,343,777)$.

In atherosclerosis, expression of $\mathrm{Cx} 37, \mathrm{Cx} 40$, and $\mathrm{Cx} 43$ varies throughout the progression of the disease (343) and the connexins play different roles in plaque development (Fig. 10). Heterozygous $\mathrm{Cx} 43$ knockout mice present with reduced and more stable atherosclerotic plaques compared to homozygous mice $(345,750)$, which indicates a promoting role of Cx43 in the development of atherosclerosis. In contrast, both Cx40 (83) and Cx37 (752) protect against plaque development. Cx37 exerts its atheroprotective effect through regulation of monocyte and macrophage recruitment (752), whereas $\mathrm{Cx} 40$ protects the vessels by preventing leukocyte adhesion (83). That $\mathrm{Cx} 37$ plays an essential role in atherosclerosis is further supported by the findings that a polymorphism of the $C \times 37$ gene is related to both coronary artery disease $(273,764)$ and myocardial infarction $(386,751)$.

Gap junctions between ECs and, to a lesser degree, between the smooth muscle cells facilitates longitudinal signaling along the blood vessels, thereby mediating socalled 
conducted vascular responses (240). When vessels are stimulated by agents that modulate the membrane potential, the change in membrane potential is spread via gap junctions along the vessel (604). Depending on whether the applied drug hyper- or depolarizes the membrane, the result is conducted vasodilation or constriction. The response travels over a considerable length of the vessel, and may be observed more than $2 \mathrm{~mm}$ from the local stimulation site $(240,605)$. Although both the VSMCs and the ECs express gap junctions, the ECs, which primarily express $\mathrm{Cx} 40$, provides the main path for the conduction process (133).

The presence of myoendothelial gap junctions allows for the passage of current between the smooth muscle cells and ECs, and these two cell layers are tightly coupled electrically (758). Besides their role in conducted responses, myoendothelial gap junctions may play an important role in endothelialmediated vasodilation. Agonist-induced hyperpolarization of ECs may spread through myoendothelial gap junctions to the vascular smooth muscle layer causing hyperpolarization and smooth muscle relaxation (133). Thus, myoendothelial gap junctions may contribute to the endothelium-derived hyperpolarizing factor (EDHF) phenomenon, although other mechanisms also contribute to endothelial-derived hyperpolarization (588).

Vasoconstriction is associated with increases in the intracellular $\mathrm{Ca}^{2+}$, due, at least in part, to IP3-mediated release of $\mathrm{Ca}^{2+}$ from intracellular stores. It is becoming evident that IP3 may diffuse across the myoendothelial gap junctions also causing $\mathrm{Ca}^{2+}$ release in the ECs (588). This will result in increased production of NO and EDHF, which will counteract the agonistinduced vasoconstriction (588).

Knockout of Cx45 in mice results in a lethal phenotype where the animals die in utero due to abnormalities of vascular development (336). Differentiation and positioning of ECs are normal, but subsequent development of the vascular system is impaired in several tissues (336). Cx40 deficient mice have impaired conducted vasodilator responses and are hypertensive (134). The hypertension is not due to impaired conducted responses per se, but rather due to dysregulation of the renin-angiotensin system $(340,730,731)$. Neither Cx40 nor Cx37 deficient mice show any abnormalities of vascular development.

\section{Digestive system}

The gastrointestinal tract-Gap junctions are highly expressed especially in the inner circular layer of intestinal smooth muscle, forming a syncytium that coordinates the contractile activity of the gut (120). Interestingly, both the circular and the longitudinal layers have been found to be both electrically and dye coupled, although it has not been possible to demonstrate the presence of gap junctions in the outer longitudinal layer of smooth muscle $(120,165,775)$. It has been suggested that the interstitial cells of Cajal (ICC) may indirectly mediate the coupling in the outer muscular layer (390). The ICC are the pacemaker cells in gastrointestinal muscles, and they also mediate or transduce inputs from enteric motor nerves to the smooth muscle syncytium $(585,586,737)$. The ICC are interconnected by gap junctions, and, in addition, are electrically and dye coupled to the smooth muscle cells $(36,37,390)$. Surprisingly, the current evidence suggests a paucity of gap junctions between the ICC and the smooth muscle cells, and the issue of how electrical and dye coupling are achieved in the gastrointestinal network of ICC and smooth muscle cells remains controversial (119). In Hirschprung's disease part of the gut is aganglionic and lacks normal motility. The expression of $\mathrm{Cx} 43$ is severely reduced or absent in ICC and smooth muscle cells in the aganglionic part of the gut, which suggests that decreased levels of gap junctions may be partly responsible for the disturbances of colon motility characteristic of the disease (472). 
Salivary glands-Little is known about the role of gap junctions in salivary gland function. The acinar cells express $\mathrm{Cx} 26$ and $\mathrm{Cx} 32$ suggesting that gap junctions may play a role in the secretory function $(339,457,623)$. In contrast, the myoepithelial cells that surround the acini express $\mathrm{Cx} 43$, which imply a role for gap junctions in coordinating the contractile function of these cells (457).

Pancreas-Like in the salivary glands, the exocrine part of the pancreas also express Cx26 and $\mathrm{Cx} 32$ in the acinar cells and, like in the salivary glands, it is assumed that gap junctions coordinates the secretory response of the cells within an acini $(428,660)$. However, the function of gap junctions do not appear to be critical for the secretory function, since isolated acinar cells has an intact secretory function (81).

In the endocrine islets of the pancreas, the $\beta$-cells are coupled by gap junctions made of Cx36 (608, 609). Several lines of evidence suggest that cell-cell coupling is essential for the normal release of insulin. Isolated islets show a normal response to stimulation by glucose whereas isolated $\beta$-cells show an increased basal release of insulin, lack of response to glucose stimulation, decreased basal expression of the insulin gene, decreased proinsulin biosynthesis, and a blunted increase in intracellular $\mathrm{Ca}^{2+}$ in response to glucose stimulation, and these effects are partially reversed by reaggregation of the cells (476). A very similar phenotype is seen in Cx36-deficient mice (548). The general pattern is disruption of normal Cx36 function, which results in an increased basal release of insulin, a lack of further insulin secretion following physiological levels of glucose stimulation, lack of synchronization of the normal intercellular $\mathrm{Ca}^{2+}$ transients following glucose stimulation, and disappearance of the normal pulsatile release of insulin despite the presence of morphologically normal islets $(29,548)$. Although the molecular mechanism whereby gap junction-mediated coupling influences islet function and insulin secretion is unknown, there is strong experimental evidence in favor of a central role of $\mathrm{Cx} 36$ in the control of islet function and insulin release in rodents $(29,476)$. In a recent study in humans, Cx36 mRNA was found to correlate with the expression of the insulin gene in the islets of both control and type 2 diabetics. This suggests that $\mathrm{Cx} 36$ contributes to the control of $\beta$-cell function by modulating gene expression (608).

Cx36 may also play a role in the pathogenesis of diabetes. The gene coding for Cx36 (GJA9) is located at chromosome $15 \mathrm{q} 14$, which is a locus found to be associated with type 2 diabetes in humans $(34,110,453)$. Furthermore, there are data to suggest that at least in mice, Cx36 may protect pancreatic $\beta$-cells against cytotoxic insults $(29,476)$.

Liver-Hepatocytes are both electrically and dye coupled due to the presence of Cx26 and Cx32 in the cells $(129,364,473,690)$. The electrical coupling synchronizes the electrical responses in hepatocytes to agonists such as glucagon (364). Gap junctions are also required for the propagation of agonist induced $\mathrm{Ca}^{2+}$ waves across populations of hepatocytes. The propagation appears to be mediated by IP3 diffusing across the gap junctions, releasing intracellular $\mathrm{Ca}^{2+}$, and activating phospholipase $\mathrm{C}$ in the neighboring cells $(96,157,566$, 575). The latter functions as a regenerative mechanism, allowing the $\mathrm{Ca}^{2+}$ wave to propagate across an entire hepatic acinus. The functional importance of the gap junctional coupling is illustrated by the observation that hepatocytes from Cx32 deficient mice show a strong reduction in IP3 permeability, which is associated with drastic reductions in glucose release in response to nerve stimulation and to agonists like glucagon and norepinephrine $(471,475,665)$. 


\section{Reproductive system}

Female reproductive system-Gap junctions are abundantly present in many tissues of the female reproductive system. Connexins are present in the smooth muscle cells of the oviduct and in the uterus, where they play a role in determining the hormonal-dependent motor activity in these tissues $(43,202,264,560)$. The amount of gap junctions in the myometrium increases at the time of labor, and their appearance are necessary for coordinating the contractile activity of the uterine smooth muscle required for the expulsion of the fetus $(197,198,200,201,202,203,440,633)$. The expression of gap junctions in the uterine smooth muscle is controlled by endocrine factors with estrogens enhancing the expression and progesterone suppressing the expression of gap junctions $(199,232,260$, $406,407,408,533,563$ ) (for more information see section on expression and trafficking). A very similar mechanism operates in the oviduct, where the number of gap junctions in the smooth muscle cells and the motor activity are increased by estrogens and decreased by progesterone $(264,560)$. These effects are likely to play an important role in propelling the ovum toward the uterine cavity following ovulation.

Gap junctions play an intriguing role in the communication between the maturing oocyte and the surrounding granulosa cells, an interaction that is required for normal follicular development (309). The granulosa cells surrounding the oocyte are interconnected by gap junctions, where the main connexin is $\mathrm{Cx} 43$ (230). The presence of $\mathrm{Cx} 43$ is required for normal development and maturation of the follicle. Follicles from $\mathrm{Cx} 43$-deficient mice are morphologically abnormal, they fail to achieve meiotic competence, and they cannot be fertilized in vitro (1). The ovarian phenotype in $\mathrm{Cx} 43$ deficient mice is very similar to that seen in animals lacking oocyte-specific protein growth differentiation factor 9 (GDF9), a paracrine factor necessary for normal follicular growth and maturation $(214,309)$. It has been suggested that, as the follicle grows, the outer layers of the follicle will be at an increasing distance from the oocyte and thus be exposed to lower concentrations of GDF9. Gap junctions may be necessary for relaying GDF9-induced signaling molecules to the most distal granulosa cells, coordinating follicular growth (309). The oocyte is coupled to the granulosa cells via homotypic Cx37 containing gap junctions $(631,719)$. Mice lacking Cx37 are infertile, and their follicles do not mature normally (631). The granulosa-oocyte coupling allows transfer of metabolites and signaling molecules between the granulosa cells and the oocyte necessary for the normal development of the follicle. The amount of $\mathrm{Cx} 43$ is increased prior to ovulation due to stimulation by FSH (230). It is thought that the efficient metabolic coupling in the follicle allows the transfer of cGMP from the granulosa cells to the oocyte, and that this is essential for preventing the oocyte from completing meiosis $(478,592)$. Just prior to ovulation there is a surge of $\mathrm{LH}$, which elicits a MAP kinase dependent phosphorylation of $\mathrm{Cx} 43$ and a decreased $\mathrm{Cx} 43$ expression, which disrupts the gap junctional coupling in the wall of the follicle and thus stops the flow of cGMP to the oocyte allowing it to complete meiosis at the time of ovulation $(478,592)$. It is of interest to note that the immature oocyte lacks mechanisms for $\mathrm{pH}$ regulation, and apparently relies on transport mechanisms in the surrounding granulosa cells for this purpose, with gap junctions establishing the necessary coupling between the two cell types $(181,182)$. There is no direct evidence linking gap junctional dysfunction to infertility in humans. It has, however, been reported that in granulosa cells from patients undergoing in vitro fertilization, Cx43 levels and intercellular conductances are positively correlated with embryo quality as judged by cleavage rate and morphology, and Cx43 levels are significantly higher in patients who becomes pregnant than in those who do not (732).

The male reproductive system-Various connexins are expressed throughout the tissues of the male reproductive system, with $\mathrm{Cx} 43$ as the predominant isoform $(525,526)$. The androgen producing Leydig cells, which are present in the interstitium of the testis, 
show both electrical and dye coupling mediated by $\mathrm{Cx} 43$-based gap junctions $(62,111,511$, $564,715)$. There is only limited information available regarding the hormonal regulation of gap junction expression in Leydig cells, but is has been reported that human chorionic gonadotropin reduced Cx43 mRNA and protein levels (770). In addition, in the Leydig cell line TM3 stimulation of testosterone secretion by LH was associated with a decreased coupling, an effect that was mimicked by activators of PKA and PKC (220). Together these data suggest that gap junction coupling may play a role as modulators of hormone secretion.

Gap junctions composed of $\mathrm{Cx} 43$ connect both Sertoli cells and germ cells in the seminiferous epithelium. These junctions are seen between Sertoli cells and between Sertoli cells and germ cells, primarily spermatogonia, and early and late spermatocytes (137). Although the underlying molecular mechanisms are unresolved, it appears as if $\mathrm{Cx} 43$ is necessary for normal spermatogenesis (526). Thus, $\mathrm{Cx} 43$ deficient mice has a 50\% reduction in primordial germ cells in the foetal testis (299) and testicular tissue from these mice show a persistent reduction in germ cells when grafted under the kidney capsule of adult male mice (570). Recently, similar observations have been made in Sertoli cell specific conditional Cx43 knockout mice $(63,651,652)$. The male mice showed an arrest of spermatogenesis at the level of spermatogonia or a Sertoli cell only syndrome with absence of germ cells. The function of $\mathrm{Cx} 43$ is specific, since other connexins are unable to compensate for the loss of $\mathrm{Cx} 43$ function $(524,748)$. As in the Leydig cell, the expression of $\mathrm{Cx} 43$ in the Sertoli cells is subject to hormonal regulation. Androgens and estrogens have been found to disrupt gap junctions and reduce the coupling in Sertoli cells $(266,523,526)$, whereas thyroid hormones increases the levels of $\mathrm{Cx} 43$ in Sertoli cells $(216,658)$. Retinoids are of importance for Sertoli cell function and spermatogenesis, and it is possible that this may, at least partly, be due to its effects on Cx43 expression in Sertoli cells $(28,92,401)$.

\section{Nervous system}

The central nervous system-Gap junctions are present in neurons and glia cells throughout the nervous system $(103,104,192,465,466,489,545,546,547,625,757)$. In mammalians, Cx36 is expressed in neurons throughout the brain, and it appears to be present exclusively in neuron-neuron gap junctions, and not in gap junctions between glial cells $(103,546,547)$. Other connexins have also been described in neurons, for example, Cx45 and Cx62 (mCx57), although they all have a much more limited distribution compared to that of Cx36 $(194,641)$. Although gap junctions between neurons and between glia cells are common, gap junctions between neurons and glia cells are uncommon (547). Gap junctions provide an additional form of contact between neurons in addition to the well-known chemical synapse. They form an electrical synapse, which in contrast to the chemical synapse, allows bidirectional signaling between neurons. A striking finding is that not all neurons have electrical synapses; rather they are present in specialized subpopulations, for example, inhibitory interneurons (192). The precise function of electrical synapses is not known, but they may play an important role in generating or modulating synchronous activity in groups of neurons $(69,136,189,279,398,692,786)$. The dynamics of electrical synapses results in a function resembling a first-order lowpass filter, and as a result they will reduce high-frequency noise, and thus, improve the signal-to-noise ratio in neural signaling pathways $(47,104)$. In addition to providing electrical coupling, gap junctions may also be important for specific neuron-to-neuron passage of small molecules, although little evidence exists for this in the mature brain. In view of the widespread occurrence of gap junctions in neurons, it is noteworthy that only limited neurological dysfunction has been observed in knockout animals. In $\mathrm{Cx} 36^{-/-}$mice there appears to be a subtle effect on hippocampal gamma oscillations, but apart from the visual defects (see later), there is little or no neurological phenotype $(69,316)$. A very similar pattern was seen in mice with a conditional neuron directed knockout of $\mathrm{Cx} 45$. The major neurophysiological effect was a disruption of 
kainite induced gamma oscillations in the hippocampus (786). The behavioral phenotype was minimal, although an effect on novel object recognition was reported (786).

Among glial cells there are abundant gap junctions between the astrocytes themselves, fewer between the astrocytes and oligodendrocytes, and very few or none between the oligodendrocytes $(300,420,465,547)$. Astrocytes and oligodendrocytes express a distinct collection of connexins, with Cx30.2 (mCx29), Cx32 and $\mathrm{Cx} 47$ being present in oligodendrocytes and Cx30 and Cx43 being present in astrocytes (489). Consequently, gap junctions between oligodendrocytes and astrocytes will always be heterotypic. Mutations or knockout of Cx32 and Cx47 results in disorders of myelination. Whereas loss of Cx32 predominantly results in demyelination of peripheral nerves (see later), loss of function of Cx47 leads to a devastating dysmyelinating disease in humans, Pelizaeus-Merzbacher-like disease (697). In mice, knockout of $\mathrm{Cx} 47$ also results in demyelination in the CNS, but the demyelination is less severe in mice compared to humans $(431,480)$. Mouse with a double knockout (Cx32 and Cx47) develops a severe dysmyelinating phenotype (431, 480). It is therefore clear that connexins and gap junctions are of importance for the function of myelinated fibers, although the exact mechanisms leading to loss of myelination remains unknown. Neuronal activity is associated with the release of $\mathrm{K}^{+}$and an increase in the local $\mathrm{K}^{+}$concentration (105). One function of the panglial syncytium may be the disposal of extracellular $\mathrm{K}^{+}$; so-called potassium siphoning $(489,543)$. This is necessary for maintaining a low extracellular $\mathrm{K}^{+}$concentration, and thus, normal neuronal activity. By spatially buffering the released $\mathrm{K}^{+}$, and by providing a restricted pathway whereby $\mathrm{K}^{+}$can reach the blood vessels, the panglial network may play an important role in maintaining brain $\mathrm{K}^{+}$homeostasis. Myelinated fibers release $\mathrm{K}^{+}$into the space between the axon and the oligodendrocytes and it has been suggested that disturbances in the function of the panglial syncytium may lead to accumulation of $\mathrm{K}^{+}$andwater in the oligodendrocytes. The intracellular accumulation of ions andwater may be an important mechanism in the demyelinating process (543). Like in other tissues (see previous text), connexins are involved in the propagation of calcium waves across glial cells, but the physiological role of these waves in the panglial syncytium remains to be fully established (593).

The peripheral nervous system-Charcot-Marie-Tooth disease is a demyelinating condition with progressive degeneration of peripheral nerves caused by mutations in Cx32 (323). In peripheral nerves, the sheaths of myelin are formed by Schwann cells wrapping their cytoplasm several times around the nerve fiber. Cx32-based gap junctions form intracellular connections between adjacent loops in one cell, thereby providing a radial diffusion pathway that allows metabolic and nutritional support of the most distal parts of the cytoplasm $(18,746)$. Disruption of this pathway is apparently associated with Schwann cell dysfunction, leading to loss of the myelin sheath, and peripheral nerve dysfunction.

The eye-The lens is an avascular organ with an epithelial layer covering its anterior surface, and a large mass of fiber cells that forms the bulk of the organ. The lens fibers are formed from the epithelial cells at the equator of the lens, and as they move inward they gradually lose their organelles to become mature lens fibers. The differentiating fibers extend from the surface and approximately $15 \%$ of the distance into the lens; and the mature fibers forms the core of the lens. The entire structure is a functional syncytium due to the presence of gap junctions coupling all cells in the lens (425). The epithelial cells contain Cx43 and Cx50 (43, 116), whereas the lens fiber cells contain Cx46 and Cx50 $(315,504)$, and the presence of dye and electrical coupling between all cells of the lens have been demonstrated $(423,539)$. Gap junctions are essential to lens function by allowing a flow of current and fluid that transports nutrients into the central part of the lens (422). The driving force is created by the action of the Na-K-ATPase, which together with the passive $\mathrm{Na}^{+}$ permeability of the lens fibers creates an intracellular voltage and concentration gradient 
from the inside to the outside of the lens. The net result is an inward flow along the extracellular space carrying nutrients into the center of the lens, together with an intracellular outward flow removing $\mathrm{Na}^{+}$and water from the core of the lens (422). In the outer differentiating fibers, both $\mathrm{Cx} 46$ and $\mathrm{Cx} 50$ contribute to the coupling, whereas in the mature fibers located in the center of the lens $\mathrm{Cx} 46$ is solely responsible for the coupling $(16,224)$. In mature fibers, both $\mathrm{Cx} 46$ and $\mathrm{Cx} 50$ are truncated by removal of their CT tail by calpain (382); a modification that leads to closure of Cx50-based gap junctions, but not of Cx46-based gap junctions $(140,162)$. So although both connexins are present in the mature fibers, only Cx46-based gap junctions contribute to the coupling. (For further information of $\mathrm{Cx} 46$ and $\mathrm{Cx} 50$ truncation, see section on posttranslational regulation.)

The coupling conductance is regulated by $\mathrm{pH}$ in the outer differentiating fibers, with a decrease in $\mathrm{pH}$ rapidly closing all gap junctions in this layer (424). In contrast, gap junctions in the mature fibers lack pH sensitivity and are not closed by cytoplasmic acidification (17, $424,436,600$ ). This could be necessary for maintaining adequate coupling, since the core of the lens is acidic (26). There is an interaction between Cx46- and Cx50-based gap junctions in the lens with respect to $\mathrm{pH}$ sensitivity. In wt mice, acidification nearly abolishes cell-cell coupling (16). In contrast, in Cx50 knockout mice, the initial coupling is reduced by approximately $50 \%$, corresponding to the loss of Cx50-based gap junctions, but the remaining coupling is nearly unchanged following acidification (16). This suggests that the $\mathrm{pH}$ sensitivity is provided by $\mathrm{Cx} 50$ and that, somehow, there is an interaction between $\mathrm{Cx} 50$ and $\mathrm{Cx} 46$ with respect to $\mathrm{pH}$ sensitivity (425).

The importance of gap junctions in normal lens function is amply illustrated by the fact that mutations in $\mathrm{Cx} 46$ and $\mathrm{Cx} 50$ are one of the common causes for hereditary cataracts in humans and mice (425). In addition, Cx50 may also be of importance for the normal development of the eyes, as Cx50 knockout mice have micropthalmia $(569,745)$.

In the vertebrate retina electrical synapses, i.e. gap junctions, are found in all the five neuronal cell types present (47). Thus, there are electrical synapses between rods, between cones, between rods and cones, between horizontal cells, between bipolar and amacrine cells, between amacrine cells, between amacrine cells and ganglion cells, and between ganglion cells. They establish bidirectional electrical coupling, and their function is similar to that seen in neurons in the rest of CNS (see previous text). The predominant connexin is $\mathrm{Cx} 36$, but $\mathrm{Cx} 45, \mathrm{Cx} 50$, and $\mathrm{Cx} 62$ (mCx57) are also present in the retinal neurons (47). Only Cx36 knockout mice are known to have a visual phenotype. Although the retina showed no morphological defects, recordings showed a reduced scotopic function, consistent with reduced vision during low light conditions (236). This is consistent with the observation that electrical synapses are involved in the rod signaling pathway (236).

The inner ear-Gap junctions play a central role in hearing, as evidenced by the fact that nearly $50 \%$ of all cases of inherited neurosensory deafness is due to mutations in the genes coding for $\mathrm{Cx} 26, \mathrm{Cx} 30, \mathrm{Cx} 31, \mathrm{Cx} 32$, and possibly $\mathrm{Cx} 43$ (418). In the vast majority of these cases, the cause is a mutation in the gene coding for $\mathrm{Cx} 26(307,365,743)$. Connexins are expressed in the epithelia and connective tissues, but not in the sensory cells (the hair cells) of the cochlea. They establish a functional syncytium among these cells $(183,188)$. In the cochlea, Cx26 and Cx32 may be involved in buffering and recycling of $\mathrm{K}^{+}(183,418)$. The sensory cells of the cochlea, the hair cells, are exposed to the endolymph through their cilia, which has a high concentration of $\mathrm{K}^{+}(\sim 150 \mathrm{mmol} / \mathrm{L})$, while their cell bodies are surrounded by the perilymph, which has an ionic composition similar to the extracellular fluid (270).

Upon stimulation by sound $\mathrm{K}^{+}$enters the hair cells due to the large electrochemical gradient, causing depolarization of the cells, a step essential for the sensation of sound. $\mathrm{K}^{+}$then exits the hair cells into the perilymph, where it is taken up by the surrounding epithelial cells and 
supporting cells and transported to the lateral wall of the cochlea via the "epithelial gap junction network." The $\mathrm{K}^{+}$is then taken up by the fibrocytes of the spiral ligament, and, through the "connective tissue gap junction network," transported back to the stria vascularis, and then finally secreted back into the endolymph (270). When this recirculation is disrupted, the result is cochlear dysfunction and hearing loss. It is of interest to note that some connexin mutants that cause deafness, form channels that conduct ionic current similar to the wt, suggesting the intercellular transport of other compounds, for example, $\mathrm{IP}_{3}$, may also be important for normal hearing $(35,418,779)$.

\section{Immune system}

Despite the fact that most cells of the immune system express connexins, very little is known about the role of gap junctions in these cells (470). Immune stem cells establish gap junctions with the surrounding stromal cells in the bone marrow and these are necessary for the terminal differentiation of primary $\mathrm{T}$ and $\mathrm{B}$ cells $(82,330,444,445,528)$. Likewise, gap junctions are necessary for the further maturation of $\mathrm{T}$ and $\mathrm{B}$ cells in the thymus and in the peripheral lymph nodes, but very little information is available regarding the underlying molecular events $(444,445,470)$. B cells and antigen presenting dendritic cells may establish heterocellular gap junctions, and treatment with connexin mimetic peptides or 18aglycyrrhetinic acid reduces the secretion of immunoglobulins $(331,493)$. It has been suggested that gap junctions may be involved in antigen presentation by allowing cell-to-cell transfer of antigens, so that small peptides generated in one cell may be transported via gap junctions to neighboring cells, where they then are presented on the cell surface by MHC class 1 molecules $(469,502)$. Recently, it was reported that activation of T lymphocytes (CD4+) is associated with an upregulation of $\mathrm{Cx} 43$ expression, and that $\mathrm{T}$ lymphocytes (Th1) establishes heterocellular contact with macrophages in the tissues; and that gap junctions are of significance for clonal expansion $(39,494)$.

Gap junctions may be involved in the transmigration of leukocytes across the endothelium, but the exact nature of the gap junction-mediated interaction between leukocytes and the endothelium remains unknown $(170,492,590,774)$. An effect on leukocyte recruitment would be in agreement with observations suggesting a role for gap junction in the inflammatory response; see reference 596 for a recent review.

\section{Cell growth, migration, and gene expression}

Connexins have complex functions in controlling cell growth, migration, and gene regulation (303). For example, mutations in $\mathrm{Cx} 43$ lead to the complex phenotype of oculodentodigital dysplasia, a condition characterized by multiple developmental disturbances including dental anomalies, fused fingers, and abnormalities in the development of the eye, nervous system, bones, and many other abnormalities (505).

The mechanisms through which connexins exerts these effects are highly diverse. In some cases it is directly related to channel formation with transfer of growth and gene regulatory factors. Possible candidates are $\mathrm{Ca}^{2+}$, cAMP, and inositol triphosphate $(495,575)$. Recent data also suggest that small oligonucleotides like siRNA can pass through gap junctions and provide a direct pathway whereby one cell can influence gene activity in neighboring cells $(704,749)$. In addition to its role in forming gap junctions, connexins may also contribute directly to cell-cell adhesion independent of channel function. This may have an important role with regard to growth control. Connexins may also contribute to contact inhibition, and loss of connexins may play a role in oncogenesis $(432)$. In some cases this effect is independent of channel formation or function $(118,314,485)$. Instead, the effect may be mediated through direct interactions between the intracellular $\mathrm{C}$-terminal part of connexins and proteins of the cytoskeleton like zona occludens-1 (ZO-1), caveolin, and drebrin, in 
addition to various kinases and phosphatases $(94,153,211,265)$. Also, normal migration of cells during, for example, neurogenesis appear to depend on connexin mediated adhesion rather than direct channel formation $(94,167)$. Binding of growth promoting substances like $\beta$-catenin may be an alternative mechanism whereby $\mathrm{Cx} 43$ could influence cell growth. The growth promoting effect of $\beta$-catenin depends on its subcellular localization; binding to Cx43 may prevent it from reaching the nucleus and thus preventing its interaction with transcription factors (4). In cardiac myocytes, there is evidence for the presence of $\mathrm{Cx} 43$ in the mitochondrial membrane, where it may contribute to the release of apoptosis-promoting factors (228).

It is possible that connexin genes may act as "hubs" in gene expression networks. Data from gene array studies show that perturbation of the gene coding for $\mathrm{Cx} 43$ may have effects on the expression of hundreds of other genes in the network $(285,650)$. Clearly, such an effect has important implications for the role of connexins in the normal development of the organism.

\section{Noncoupling Roles of Connexins}

Besides their classical role as mediators of intercellular coupling, connexins also play roles that are independent of their function in cell-cell channels. The most studied example of this is the role of unpaired hemichannels in the plasma membrane, which upon opening may exert different physiological effects ranging from paracrine signaling to loss of homeostasisinduced cell death. More surprisingly, connexins have also been assigned to roles exerted in the nucleus and mitochondria as well as some channel-independent effects, which involve connexin-binding proteins. The current knowledge is reviewed in the later section.

\section{Hemichannels}

Undocked connexons in unopposed parts of the cell membrane are referred to as hemichannels. Like proper gap junction channels they may be formed by all the known connexins, and they exist in both homo- and heteromeric conformations (172). In addition to connexins, it appears likely that the recently discovered family of pannexins forms similar hexameric hemichannels in the plasma membrane $(20,66,395)$. Pannexins have a membrane topology similar to the connexins, although they have no sequence homology $(500,501)$. Pannexin hemichannels will not be dealt with further in the present text.

When arriving at the plasma membrane hemichannels may diffuse laterally in the plasma membrane and become incorporated into gap junction plaques, but it appears likely that the plasma membrane at any given time contains a significant number of undocked

hemichannels. They form large pores in the cell membrane, which upon opening allows the exchange of ions and small molecules between the intra- and the extracellular compartment. Current evidence suggests that the synthesis and intracellular trafficking of undocked hemichannels closely mimics that of the connexons, which later ends up in intercellular channels.

The amount of unopposed hemichannels in the membrane can be assessed by biotinylation of surface membrane proteins followed by "pull down" of the labeled proteins $(552,584,595)$. In Cx32-transfected HeLa cells approximately $4 \%$ of surface connexin was in unopposed hemichannels (584), whereas in cultured astrocytes approximately $15 \%$ of surface $\mathrm{Cx} 43$ was found in unopposed hemichannels (552). Metabolic inhibition, treatment with a $\mathrm{Ca}^{2+}$ ionophore or FGF-1 all resulted in increased levels of hemichannels in the surface membrane $(552,584,595)$. An increase in intracellular $\mathrm{Ca}^{2+}$ appeared to be necessary for the response and since it was not associated with an increase in the total connexin levels, it was primarily due to a redistribution of connexons in the surface 
membrane $(552,584,595)$. In HeLa transfectants, the increased levels of $\mathrm{Cx} 43$ hemichannels following stimulation with FGF-1 involved activation of p38 MAP kinase, and the data suggests that the activation of this kinase is a downstream response to the $\mathrm{Ca}^{2+}$ elevation (595).

The conductance and permeability characteristics of hemichannels are very similar to that of the full gap junction channels. Since a hemichannel represents one half of the full cell-cell channel it is to be expected that it will have twice the conductance and permeability of the full channel, and indeed this appears to be the case (576). Thus, like the cell-cell channels, an open hemichannel would be permeable to rather large molecules (up to $\sim 1 \mathrm{kDa}$ ), and it is clear that if a substantial amount of hemichannels were open under resting conditions, the cell would undergo severe derangements in electrolyte balance, membrane depolarization, and loss of small intracellular molecules like ATP. Because of these potentially fatal consequences it is generally accepted that most hemichannels are closed during resting conditions $(23,38)$. A possible exception could be Cx26 hemichannels. Expression of either human or sheep Cx26 in oocytes or Neuro2a cells, resulted in hemichannels that were open under resting conditions apparently without compromising cell viability $(226,562)$.

Interestingly, transfection with rat Cx26 did not result in expression of open hemichannels indicating significant species differences (226).

While most hemichannels are closed during resting conditions, there is now good evidence to suggest that opening of hemichannels for short periods allows exchange of molecules between the intra- and extracellular compartment, and that this exchange plays a significant role in many physiological and pathophysiological processes $(23,38,172,576,577)$.

The controversy surrounding the existence of hemichannel mediated transmembraneous fluxes stems from the fact that their presence mostly has been inferred from indirect evidence. The first evidence came from studies showing that expression of connexins in cells lead to the appearance of membrane permeabilities or currents not present in the nontransfected cells $(161,504)$. The observations that (1) such currents or permeabilities could be inhibited by gap junction blockers, (2) the induced pathways were permeable to larger molecules (up to $1 \mathrm{kDa}$ ), and 3) the currents had reversal potentials around $0 \mathrm{mV}$ indicative of a pathway with low selectivity gave further support for the presence of open hemichannels $(107,293,376,522,663)$. However, because of the poor specificity of gap junction blockers together with the fact that other ion channels may allow the transmembraneous passage of larger molecules and metabolites $(135,143,434)$ it could not be ruled out that other ion channels mediated the observed fluxes. The possibility that hemichannels may under some circumstances carry transmembraneous currents have been strengthened by single channel recordings in transfected cells. These studies have demonstrated both conductances and substates compatible with what is known from intercellular channels $(106,694,701,705,727)$.

\section{Hemichannel gating properties}

Although most hemichannels appears to be closed in the resting state, several factors may trigger their opening. Among the first to be described was a decrease in the extracellular $\mathrm{Ca}^{2+}$ concentration (161), but later it has been shown that also changes in phosphorylation status, the extracellular ion composition, membrane potential, intracellular $\mathrm{pH}$ and redox potential, and mechanical stimulation may cause opening of hemichannels $(553,576,577)$.

Extracellular cations-Reducing the extracellular $\mathrm{Ca}^{2+}$ concentration increases the open probability of all investigated connexin hemichannels $(514,576,577)$. Despite this uniform effect of lowering extracellular $\mathrm{Ca}^{2+}$, the mechanism seems to differ between hemichannels made from different connexins $(223,456,531,677,726)$. Although most hemichannels 
appear to be closed when $\mathrm{Ca}^{2+}$ is above $1 \mathrm{mmol} / \mathrm{L}$, this mechanism may be of pathophysiological significance, for example, during cerebral ischemia, where the extracellular $\mathrm{Ca}^{2+}$ level may be as low as $0.1 \mathrm{mmol} / \mathrm{L}(251)$. The extracellular levels of other divalent cations like $\mathrm{Zn}^{2+}, \mathrm{Cd}^{2+}, \mathrm{Co}^{2+}, \mathrm{Ba}^{2+}$, and $\mathrm{Mg}^{2+}$ also affects the gating properties, with the general picture being inhibition with increasing concentrations $(85,86,160,668)$. Replacing extracellular $\mathrm{Na}^{+}$by $\mathrm{K}^{+}, \mathrm{Cs}^{+}$, or $\mathrm{Rb}^{+}$causes an increase in the open probability of $\mathrm{Cx} 46$ and $\mathrm{Cx} 50$ hemichannels, suggesting that monovalent cations may interact with $\mathrm{Ca}^{2+}$ in determining the activity of hemichannels (653).

Phosphorylation-Cx43 hemichannels reconstituted in liposomes are permeable to Lucifer Yellow, and treatment with alkaline phosphates before or after the reconstitution increases the permeability whereas treatment with MAP kinase or PKC decreases it (22, 310). Phosphorylation of serine 368 may be of particular significance in this respect, since hemichannels formed by a mutated Cx43 lacking this residue (Cx43 S368A) remain in the open state (22). These data strongly suggest that phosphorylation may directly regulate $\mathrm{Cx} 43$ hemichannel activity.

Membrane potential-The available studies suggest that depolarizing the cell membrane increases the open probability of hemichannels $(141,161,289,514,703,776)$. However, as activation requires substantial depolarization of the membrane, the physiological importance of hemichannel activation by voltage remains to be demonstrated. One possibility is that interactions exist between activation by depolarization and reduced extracellular $\mathrm{Ca}^{2+}$. This has been shown for $\mathrm{Cx} 46$, where lowering of extracellular $\mathrm{Ca}^{2+}$ results in a hyperpolarizing shift in the activation curve $(514,726)$.

Chemical factors-Hemichannels composed of Cx26, Cx35, Cx38, Cx43, Cx45, and $\mathrm{Cx} 46$ have all been found to be sensitive to the intracellular $\mathrm{pH}$, with acidification leading to closure of the channels $(184,289,562,693,701,744)$. In addition to $\mathrm{pH}$, some hemichannels may also be sensitive to the intracellular redox potential. The data are less clear since both oxidation and reduction appears to be able to increase the open probability of the hemichannels. Oxidative stress increases the activity of Cx43 hemichannels, and since the effect occurs within a few seconds, it is most likely due to activation of hemichannels already present in the membrane $(488,552,553)$. The effect could be blocked by reducing agents like DTT or intracellular glutathione, or scavengers of free radicals, suggesting that it is due to oxidation of cysteine residues in the C-terminal part of $\mathrm{Cx} 43(107,552,553)$. In contrast, it has also been reported that DTT increases the open probability of Cx43 hemichannels under normoxic conditions (554). This apparent paradox has been suggested to be explained by a modulation of the sensitivity to the redox potential by progressive changes in phosphorylation status (555).

Mechanical stimulation-Various forms of mechanical stress such as direct mechanical stimulation, hyperosmotic stress, and shear stress have all been reported to increase the activity of connexin hemichannels $(21,195,292,568,629)$. Whether it is a direct effect or whether it is mediated by other mechanisms like phosphorylation remains to be determined.

\section{Hemichannel pharmacology}

Hemichannels are blocked by the same compounds that blocks intercellular channels. Thus the unspecific gap junction inhibitors heptanol, octanol, halothane, carbenoxolone, 18glycyrrhetinic, various fatty acids (e.g., arachidonic acid) and fenamate derivatives [e.g., flufenamic acid (FFA)] also all block hemichannels. All these agents have a low specificity since they block other ion channels as well. Consequently, results obtained from pharmacologic manipulation of hemichannels by these agents should be interpreted with 
caution. Lanthanides such as $\mathrm{La}^{3+}$ and $\mathrm{Gd}^{3+}$ appear to block hemichannels by entering the pore region of the channel. Like the previous agents it is unspecific in the sense that it may also block other ion channels, but in contrast to the other agents it is without effect on intact intercellular channels when added to the extracellular medium. In contrast, the connexin mimetic peptides are considered to be more specific blockers of hemichannels. They are designed to mimic parts of the extracellular loop of the various connexins, and they were originally intended for inhibiting cell-cell communication by blocking formation of new cell-cell channels, but they appear to be useful tools for inhibiting connexin hemichannels as well $(59,60,171,173,371)$. However, these results may have to be interpreted with caution since a recent study failed to show any inhibitory action of the peptides on connexin hemichannel activity (113). Instead, membrane channels formed by the unrelated pannexin 1 were inhibited. The connexin mimetic peptides may therefore be less specific than previously thought, since they may also have effects on other channels, in particular pannexin hemichannels.

Quinine has been reported to activate a series of connexin hemichannels found in different vertebrates $(147,414,415,561,744)$, although there appears to be no reports of such an effect in mammalian systems.

\section{Physiological and pathophysiological roles}

Hemichannels have been suggested to be involved in several physiological and pathophysiological processes. Most of the experimental data on hemichannels have however been obtained in vitro, and often in transfected cells that normally does not express connexins. The scarcity of data from in vivo studies calls for considerable caution when extrapolating results obtained in cell systems to processes occurring in the normal or diseased organism.

Release of ATP, NAD+, and glutamate-Hemichannels have been suggested to play an important role in the release of signaling molecules to the extracellular medium. Several studies have shown that hemichannels may be an important pathway for releasing ATP to the extracellular compartment $(7,8,59,60,100,169,174,195,204,207,222,242,301$, $305,371,373,412,458,483,503,506,568,597,636,663,781)$. The released ATP can bind to purinergic receptors on the secreting or neighboring cells, and thus act as a signaling molecule in auto- and paracrine responses. One well described effect is the initiation of $\mathrm{Ca}^{2+}$ waves, where the released ATP diffuses and acts extracellularly to cause an increase in the intracellular $\mathrm{Ca}^{2+}$ concentration, which spreads across the neighboring cells. $\mathrm{Ca}^{2+}$ waves have been described in many different cell types, for example, epithelia, nervous tissue, liver and smooth muscle cells $(166,572,587,741,771)$. The spread of $\mathrm{Ca}^{2+}$ waves across cell populations may among other things play important roles in embryogenesis by coordination the migration and differentiation of progenitor cells $(166,741)$.

Interestingly, $\mathrm{Cx} 30$ is present in the luminal membrane of renal tubular epithelial cells in the distal part of the nephron (427). Being an unopposed membrane, they must be present as hemichannels and appear to play a role in pressure natriuresis by allowing secretion of ATP into the tubular lumen with subsequent inhibition of sodium reabsorption in response to acute increases in the arterial blood pressure (636).

Hemichannels may also play an important role in the regulation of the intracellular $\mathrm{Ca}^{2+}$ concentration. Cyclic ADP ribose (cADPR) is an endogenous ligand for Ryanodine receptors and causes release of $\mathrm{Ca}^{2+}$ from intracellular stores (179). It is synthesized from $\mathrm{NAD}^{+}$by the ectoenzyme CD38 and transported back into the cell through active or facilitated transport mechanisms (298). The precursor $\mathrm{NAD}^{+}$appears to reach the ectoenzyme by release via $\mathrm{Cx} 43$ hemichannels $(67,68)$. 
Hemichannels have also been proposed as a pathway for the release of glutamate from astrocytes (762). The release was especially prominent under conditions of low extracellular $\mathrm{Ca}^{2+}$ concentrations; levels that may well occur during ischemia, seizures or spreading depression (251). Since glutamate mediated excitotoxicity is one of the major mechanisms underlying ischemic brain injury, blockade of hemichannels may be a new therapeutic target for preventing brain injury. However, these data have been challenged by more recent studies that have failed to find glutamate release through hemichannels (388).

Hemichannels and cell death-Many of the factors that regulate connexin hemichannels are found during metabolic inhibition and/or ischemiareperfusion, that is, ATP depletion, protein dephosphorylation, decrease in extracellular $\mathrm{Ca}^{2+}$ and $\mathrm{Mg}^{2+}$ concentrations, increases in intracellular $\mathrm{Ca}^{2+}$ concentration, and changes in redox potential. It is therefore likely that hemichannels may be activated during metabolic inhibition and/or ischemiareperfusion $(23,108,293,327,374,488,555,584,624,682,721)$. Opening of hemichannels may lead to collapse of the membrane potential due to disturbances in ionic homeostasis including intracellular $\mathrm{Na}^{+}$and $\mathrm{Ca}^{2+}$ gain and loss of $\mathrm{K}^{+}$. Loss of metabolically relevant compounds such as glucose, $\mathrm{ATP}, \mathrm{NAD}^{+}$, and free radical scavengers like glutathione and ascorbic acid may contribute to cellular injury and death $(2,68,542,553)$. In addition, the extracellular release of $\mathrm{K}^{+}$, glutamate, and metabolites of arachidonic acid may cause depolarization and excitotoxicity and thus paracrine mediated cell death in neighboring cells $(555,629,671,762)$. In agreement with such a role, acute knockdown of connexins or application of certain pharmacological blockers have been found to have a protective effect under ischemic conditions suggesting that opening of connexin hemichannels may contribute to cell death $(131,178,186,187,255,624,682)$. This issue is complicated by the fact that other studies indicate a protective effect of gap junctions and hemichannels during ischemic conditions $(98,329,439,597)$. Thus, $\mathrm{Cx} 43$ hemichannels has been implicated in heart pre- and postconditioning (602), and data suggest that ATP secreted via $\mathrm{Cx} 36$ and $\mathrm{Cx} 43$ hemichannels may act via purinergic receptors to induce neuroprotection during ischemia $(381,597)$. Preconditioning has been shown to reduce the degradation of Cx43 hemichannels, which leads to an increase in the amount surface hemichannels (381). Supporting the involvement of $\mathrm{Cx} 43$ in the preconditioning response, $\mathrm{Cx} 43$ null mice were insensitive to hypoxic preconditioning (381). Further studies are clearly needed to define the specific circumstances under which hemichannels may have either protective or detrimental roles.

Hemichannels in hereditary diseases-Mutations in different connexins have been implicated in several human genetic disorders, and in some cases it has been speculated that defective hemichannel function could play a role in the pathogenesis; for a recent review see reference 594.

Hemichannels in acquired human diseases-Besides their role in ischemia, which is reviewed previously, hemichannels may be involved in the pathogenesis of such diverse conditions as atherosclerosis, inflammation, and bacterial infections $(241,553,594,688$, 720). However, most of the data were obtained in vitro, and there is very little data from studies in the integrated organism. This makes it difficult to judge the clinical relevance of the observations, and it is clear that additional research is required to define the possible roles of hemichannels in human diseases.

\section{The gap junction proteome}

Several connexin-binding proteins have been identified and are collectively referred to as the gap junction proteome (for recent reviews see references 210,352). In particular, the CT domains of most connexins contain multiple sites for protein-protein interactions. Some 
connexin-binding proteins involved in trafficking, gating, or posttranslational modification of connexins are discussed in other sections of this article. In addition, connexins also interact with proteins involved in cell growth (such as the CCN3-Cx43 $(190,206)$ and Discs Large homolog 1 (Dlgh1)-Cx32 interaction (154)) as well as receptor and channel proteins such as the acetylcholine receptor (AChR) (773) and aquaporin-0 (AQP0) (389). The variety of proteins that interact with connexins, leaves no doubt that connexins have several channel-independent effects. However, the gap junction proteome is not yet complete and as more connexin-binding proteins may be identified in the future, additional channelindependent effects of gap junctions may also be described.

\section{Connexins regulate cell growth independent of coupling}

Connexin expression is capable of regulating cell growth (759) and connexins have been hypothesized to play a role in cancer development (for review see (reference 112)). The link between gap junctions and cell growthwas originally proposed by Loewenstein $(396,429)$ and the idea is supported by many subsequent studies. Suppression of cell growth can be obtained by regulation of gene expression and several genes involved in the cell cycle or cell cycle repression have been implicated (112). The effect of connexins on cell growth is probably mediated by both coupling-dependent and couplingindependent components (112). However, reintroduction of connexin in tumor cells can suppress growth without increasing coupling $(281,534)$. For example, growth suppression is observed in cultured cells without cell-cell contacts (446), cells cultured with GJ inhibitors (446), cells expressing connexins that are incapable of forming functional channels (484), and cells expressing only CTfragments of connexins $(117,446,780)$. Therefore, it seems that coupling-independent effects are of major importance.

Cx43 is by far the most studied connexin with regard to growth suppression, but other connexins, such as Cx26 (433), Cx30 (529), Cx32 $(53,430)$, have been shown to have similar effects; whereas other studies show that Cx32 (433) and Cx40 $(205,433)$ do not. The overlap for Cx32 suggests that the effect may depend on both cell type and setting. This holds true also for $\mathrm{Cx} 43$, which can be highly expressed even in very tumorigenic cells (635).

In the case of $\mathrm{Cx} 43$, growth suppression is likely caused by the $\mathrm{CT}$, since expression of $\mathrm{Cx} 43-\mathrm{CT}$ is equally efficient in suppressing growth compared to full length channels and since Cx43 lacking the CT does not affect cell growth even though they form functional channels $(446,780)$. The inhibitory effect is reported to depend on phosphorylation, and mimicking constitutive phosphorylation of S262 prevents growth suppression (118). The basis of the inhibitory effect is possibly linked to a nuclear localization of connexins. Expressing the $\mathrm{Cx} 43-\mathrm{CT}$ in cardiomyocytes and HeLa cells results in a strong nuclear localization (117), but notably this does not occur for the full length Cx43. However, the Cx43-CT may play a physiological role under certain circumstances. Taffet and co-workers identified a $20 \mathrm{kDa}$ CT fragment in several cell lines that accounted for up to $65 \%$ of the total Cx43 content (full length plus CT fragment) (297). The authors could not detect the fragment in native rat tissue; however, this does not rule out a role for the CT fragment under special circumstances. It is certainly tempting to speculate that induced cleavage of Cx43 could regulate cell growth, though the CT may also exert its effect while part of the full length protein. For example, $\mathrm{Cx} 43$ regulates the transcription factor NOV. Increased Cx43 expression not only upregulates the transcription factor NOV but also mediate NOVs translocation from the nucleus to the cell membrane by direct binding to the CT (190, 205, 239). Expression of CT-truncated $\mathrm{Cx} 43$ does not affect NOV expression or localization, showing that the effect is CT specific $(190,205)$. 
Future studies are needed to elucidate the role of connexins in growth regulation and determine whether their CTs act as transcription factors, alter transcription by binding factors like NOV, initiate signal transduction, or something entirely different.

\section{Mitochondrial connexin}

The mitochondrial localization of Cx43 was originally reported in cultured ECs by a combination of immunostaining and western blot (375). Treatment with homocysteine increased the amount of $\mathrm{Cx} 43$ in mitochondria without increasing intercellular coupling. Homocysteine treatment also reduced the EDHF-mediated vasodilatory response to acetylcholine and the authors hypothesized that sequestering of $\mathrm{Cx} 43$ from gap junctional plaques reduced EDHF signaling between the endothelium and smooth muscle cells.

Around the same time, it was shown that the cardioprotective effect obtained by ischemic preconditioning was lost in mice with a heterozygous knockout of $\mathrm{Cx} 43\left(\mathrm{Cx} 43^{+/-}\right)(603)$. The mice have a 50\% reduction in $\mathrm{Cx} 43$ expression, but exhibit normal action potential propagation due to overcapacity in coupling known as the conduction reserve (709). Therefore, it was deemed unlikely that reduced intercellular coupling per se eliminates preconditioning, which is supported by the finding that preconditioning is also lost in isolated cardiomyocytes (no cell-cell contact) from $\mathrm{Cx} 43^{(+/-)}$mice (377). Preconditioning is induced by short intermittent episodes of ischemia and reduces infarct size during subsequent prolonged ischemia (459). Many processes and signaling mechanisms have been implied in preconditioning and mitochondria play a central role. Cell death by necrosis is often associated with mitochondrial swelling and rupture, which deprives the cell of energy production and exposes it to $\mathrm{Ca}^{2+}$ overload, which in the case of the cardiomyocyte leads to disruptive hypercontracture. Apparently, preconditioning primes the mitochondria and makes them resistant to ischemia. So if $\mathrm{Cx} 43$ plays a noncoupling role in preconditioning, they could do so from the mitochondria. Schulz and co-workers demonstrated that $\mathrm{Cx} 43$ is indeed found in mitochondria in both rats, mice, pigs, and humans (49), but apparently only in mitochondria located just below the sarcolemma and not in those lying deeper between the myofibrils (51). Mitochondrial $\mathrm{Cx} 43$ (mito-Cx43) have been reported to reside in both the outer $(228)$ and inner $(49,567)$ membranes of mitochondria, and several lines of evidence show that the Cx43-CT is most likely located in the intermembrane space (51). Cardiac mito-Cx 43 content is acutely increased by preconditioning $(49,399)$ and homocysteine (696), whereas hyperglycemia reduces mito-Cx43 in the retina (441). Some information has come forth about the route of $\mathrm{Cx} 43$ to the mitochondria. $\mathrm{Cx} 43$ does not contain mitochondria-targeting sequences as such, but enters the mitochondria via the translocase of the outer membrane (TOM) by binding to chaperones. Cx43 interacts with the chaperone Hsp90 and Tom20, and inhibition of Hsp90 by geldanamycin prevents $\mathrm{Cx} 43$ translocation to the mitochondria (567).

The role of mito-Cx43 in preconditioning is not entirely clear but is thought to involve formation of reactive oxygen species (ROSs) and activation of mitochondrial $\mathrm{K}_{\mathrm{ATP}}$ channels (mito- $\mathrm{K}_{\mathrm{ATP}}$ ). Preconditioning involves ROS formation, which can be stimulated by the $\mathrm{K}_{\mathrm{ATP}}$ opener diazoxide (497). Diazoxide-induced ROS formation is reduced in $\mathrm{Cx} 43^{(+/-)}$ myocytes, whereas direct application of ROS formation still offers protection, indicating that Cx43 is working upstream of ROS formation (258). Consistent with this, inhibition of Cx43 translocation to the mitochondria by geldanamycin also prevents diazoxide-induced protection (567). However, geldanamycin does not prevent preconditioning induced by short pulses of ischemia, indicating that parallel $\mathrm{Cx} 43$-dependent signaling mechanisms are involved and that these mechanisms may have different thresholds regarding the amount of mito-Cx43 needed. 
Diazoxide activates the mito- $\mathrm{K}_{\mathrm{ATP}}$ channel that also resides in the inner mitochondrial membrane. Using patch clamp on isolated preparations of inner mitochondrial membranes, it was shown that the protective effect of diazoxide was mediated by PKC- $\epsilon$ activation and that this activation was entirely dependent on the presence of $\mathrm{Cx} 43$ because mito- $\mathrm{K}_{\mathrm{ATP}}$ activation was reduced or abolished by hetero and homozygous $\mathrm{KO}$ of $\mathrm{Cx} 43$ (573). Interestingly, the same study also showed that several blockers of Cx43 hemiand gap junctional channels also prevented PKC activation of mito- $\mathrm{K}_{\mathrm{ATP}}$ channels, implying that Cx43 permeation may be needed.

Several lines of evidence indicate that the protective role of $\mathrm{Cx} 43$ in the mitochondria may be unique to this connexin. Mitochondrial $\mathrm{Cx} 43$ has not been investigated in many noncardiac tissues, but the gap junction inhibitor carbenoxolone inhibits opening of the permeability transition pore in mitochondria isolated from astrocytes (Cx43 expressing) but not in mitochondria from liver cells (expressing Cx26 and Cx32) (15). The reason for the lack of protection by $\mathrm{Cx} 32$ could be either that it does not offer protection per se and/or because it simply does not translocate to the mitochondria. In favor of the latter, mice in which the $\mathrm{Cx} 43$ coding sequence is exchanged for that of $\mathrm{Cx} 32$ express $\mathrm{Cx} 32$ in the myocardium, but $\mathrm{Cx} 32$ is not present in mitochondria as $\mathrm{Cx} 43$ is in wt mice (437).

Even though the exact mechanisms are currently being unraveled there is good evidence that ischemic stress promotes the transport of $\mathrm{Cx} 43$ to the mitochondria and that mito-Cx43 protect the mitochondria from rupturing and killing the cardiomyocytes. Interestingly, this ability seems to be lost with age, where the amount of both $\mathrm{Cx} 43$ and mito-Cx43 is reduced (50) and possibly the mechanism is only for protection in the young. However, the mechanism is not universal to all tissues expressing $\mathrm{Cx} 43$ and as described in the section on hemichannels, $\mathrm{Cx} 43$ may protect neurons in an auto/paracrine manner by acting as hemichannels. It will also be interesting to see if other connexins than $\mathrm{Cx} 43$ translocate to the mitochondria and offer similar protection.

\section{Pharmacology of Gap Junctions}

Pharmacological agents aimed at modifying (in fact, impairing) the function of ion channels have existed for a number of years. Their use has improved the life of many and perhaps, impaired the life of some. In the case of antiarrhythmic therapy, for example, pharmacological development has been based on making a disrupted electrophysiological substrate, worse. For the most part, agents that seek to treat cardiac arrhythmias are designed to block, rather than improve, ion channel function. Interestingly, drugs exist to interfere with currents mediated by various ion channel proteins: sodium, calcium, or potassium of various kinds; but for reasons that combine luck and technical difficulties (reasons that are found throughout the history of therapeutics), gap junctions have been spared from clinical application as pharmacological targets. At present time, there are no drugs in clinical use that selectively modify gap junction-mediated intercellular communication. However, there is active basic and translational research that seeks to develop such an application. In this section, we briefly review the current status of gap junction pharmacology.

\section{Chemical agents that induce gap junction closure}

Long-chain alcohols, fatty acids, and general anesthetics-In an elegant paper published in Nature, 1980 (295), Johnston, Simon, and Ramon first reported that anesthetics, and in particular long-chain alcohols, would cause closure of gap junction channels. These early experiments were followed by others, showing that myristoleic acid, decanoic acid, and palmitoleic acid are all capable of uncoupling cardiac gap junction channels, concurrent with a decrease in open channel probability $(77,670)$. Similarly, halothane and isoflurane are two general anesthetics that reversibly close gap junctions at concentrations used in 
clinical practice (78). Follow-up experiments indicated that, though these agents close gap junctions, they also impair function of several other ionic currents $(78,512,626)$. It has been proposed that the mechanism of action of these agents is rather nonspecific, perhaps by disrupting the fluidity of the plasma membrane, and/or affecting the hydrophobic lipidprotein interface (27). Yet, studies in other ion channels suggest that general anesthetics interact directly with the channel protein, likely within selected binding pockets in poreforming domains that are, on the other hand, shared by various molecules, thus explaining their lack of selectivity for the connexin channels, in particular $(392,589)$.

Glycyrrhizic acid metabolites-Davidson et al. used a metabolic cooperativity assay in human fibroblasts to determine that the glycyrrhizic acid metabolites 18-a-glycyrrhetinic acid, 18- $\beta$-glycyrrhetinic acid ( $\alpha-$ and $\beta G A$ ), and carbenoxolone reversibly inhibited coupling in concentrations as low as $2 \mu \mathrm{mol} / \mathrm{L}$ (125). These compounds require a longer exposure time than alkanols and general anesthetics. Their mechanism of action remains unclear, though it has been proposed that they may affect the phosphorylation state of the protein, or modify the ability of connexin subunits to aggregate within the cellular environment $(125,126)$. A study by Sagar et al. further investigated the effects of carbenoxolone on junctional dye transfer in bovine aortic ECs (BAECs), which express Cx43 (582). The authors found that staurosporine inhibited the carbenoxolone-induced increase in Cx43 content. This result suggested a role for kinases in the mechanism of carbenoxolone. As with other compounds, the effect of these metabolites is not selective for gap junctions (582).

\section{The phosphorylation state of connexin as a pharmacological target-Gap}

junctions are regulated by a variety of kinases, as well as by activation of pertinent phosphatases (for additional information, see section on Cx phosphorylation). In the particular case of $\mathrm{Cx} 43$, it has been postulated that modification of its phosphorylation state may affect its ability to associate with partner molecules and/or to function as a channelforming protein (see, e.g., references $44,550,712$ ). Kinase activity could then be seen as a potential path for modifying intercellular communication. In fact, recent studies have suggested that some of the peptides said to preserve the open state of gap junctions, do so via alteration of kinase pathways (see reference 14). Yet, it is important to emphasize that kinases are, in general, highly promiscuous enzymes that catalyze the phosphorylation of multiple substrates. From this perspective, it is difficult to conceive that molecules that alter kinase activity could be developed as selective modifiers of gap junctionmediated intercellular communication.

Antimalarials-The effects of the antimalarials on gap junction coupling, particularly quinine and quinine-derivatives-like mefloquine, are interesting because they seem to be connexin-subtype specific. A study by Srinivas et al. showed that quinine dramatically blocks channels formed by $\mathrm{Cx} 36$ and $\mathrm{Cx} 50$, and moderately blocks $\mathrm{Cx} 45$ channels (shown by a $50 \%$ decrease in gap junction conductance), whereas the other connexin subtypes were insensitive to quinine exposure (654). This study represented the first demonstration of connexin-specific gap junction block by a pharmacological agent.

Fenamates-These nonsteroidal drugs are generally known for their antiinflammatory effects at nanomolar concentrations, but at higher concentrations $(10-100 \mu \mathrm{mol} / \mathrm{L})$, they were shown to block membrane ion channels $(247,601)$. These studies were followed by others, showing that these compounds have uncoupling effects in monolayer cultured cells expressing gap junction channels (247). Harks et al. showed that flufenamic acid (FFA), niflumic acid (NFA), and meclofenamic acid (MFA) caused uncoupling in Cx43-expressing NRK cells. The block was rapid, but not entirely reversible. The order of potency was 
MFA $>$ NFA $>$ FFA for block of electrical and dye coupling (247). The work was followed and expanded by the elegant studies of Srinivas and Spray demonstrating that FFA does not greatly discriminate between connexins, and that the effect does not seem to be mediated by direct binding of the drug to the pore of the gap junction channel. Single channel recordings indicated that FFA reduced channel open probability without modifying current amplitude, a fact consistent with other gap junction blockers (655).

Cardiac glycosides-The observation that cardiac glycosides strophanthidin, ouabain, and digitoxin decrease intercellular coupling attracted a great deal of attention at a time when the medical use of digitalis derivatives was still quite extensive (130). Reports that followed the early studies suggested that the effect likely involved an increase in the concentration of intracellular $\mathrm{Ca}^{2+}(426)$. These studies have not been pursued further in recent years, perhaps given the decreasing use of these agents in the medical practice. Yet, it remains possible that the structural characteristics of the cardiac glycoside molecules could bring some clues as to the structure of a pharmacophore with selective action on the gap junction molecules.

Molecular approaches to reduction of cell-cell coupling-While the agents described previously reduce coupling in a rather nonselective manner, some investigators have specifically targeted Cx43 RNA by antisense-directed strategies. Small oligonucleotides (typically 20 bases or fewer) have been shown to effectively decrease the amount of gap junction channel protein expressed. In an initial study, Qiu et al. correlated the level of local Cx43 expression with the time course of skin wound healing in mouse (536). These authors showed that a single topical application of Cx43 antisense gel brought about a transient downregulation of $\mathrm{Cx} 43$ protein levels, ultimately resulting in a dramatic increase in the rate of skin wound closure. The results showed a dampened inflammatory response and a simultaneously enhanced rate of reepithelization. Microscopically, a significant reduction in neutrophil numbers was seen in the tissue around the wound. The added therapeutic benefits included a significant reduction in the extent of granulation tissue deposition and the subsequent formation of a smaller, less distorted, scar. The studies of Law et al. are consistent with these observations (362). Whether reduction in Cx43 levels could change the time course and formation of scar in other tissues (such as heart) remains to be determined. It is worth noting that decreased $\mathrm{Cx} 43$ expression (in this case by gene haplodeficiency) also decreased the progression of atherosclerotic plaque formation in a murine model of the disease (345).

\section{Peptide strategies for reduction of gap junction-mediated intercellular communication-In 1994, Dahl et al. (115) and Monaghan et al. (443) showed that} extracellular application of peptides corresponding to relevant extracellular domains of connexins decreased the extent of cell-cell coupling. Specifically, synthetic peptides representing the extracellular loop sequences of Cx32 inhibited cell-cell channel formation (115). The advantages and limitations of the use of these "connexin mimetic peptides" were reviewed by Evans et al. (172). These authors pointed to the fact that these molecules could block not only Cx37, Cx40, and Cx43 channels but also connexin hemichannels. Various groups have shown these peptides to effectively inhibit gap junctional transfer of fluorescent dyes, electrical coupling, and synchronized $\mathrm{Ca}^{2+}$ oscillations in smooth muscle cells (172). The two most widely used peptides include Gap 26 and Gap 27, which correspond respectively to sequences on the first and second extracellular loops of $\mathrm{Cx} 43$. These two peptides have also been shown to inhibit the intercellular propagation of $\mathrm{Ca}^{2+}$ waves in monolayer cell cultures (172). Yet, additional studies suggest that these peptides may not be as specific as originally thought. Indeed, in examining the effects of connexin and pannexin mimetic peptides, Wang et al. (734) showed an attenuation of channel currents that was not 
consistent with sequence-specific actions of the peptides. They found that connexin mimetic peptides inhibited pannexin channel currents, but did not inhibit the connexin currents of the channels from which the sequence was derived. Similarly, pannexin mimetic peptides did inhibit pannexin channel currents, but also extended promiscuously to $\mathrm{Cx} 46$ channels (734).

\section{Agents that increase gap junction coupling}

In the previous sections, we have described agents known to decrease coupling (albeit with limited specificity). Less is known about molecules that could increase electrical communication in the heart. Though some kinases can improve gap junction-mediated coupling, their action can be hardly argued as specific, as mentioned before. Indeed, all of these agents have nonconnexin molecules as targets, and act on complex intracellular signaling pathways that affect a number of cellular processes. Specificity of targeting remains an important challenge in connexin pharmacology today. As with the use of connexin-mimetic molecules, peptide-based strategies have been developed to identify molecules that can enhance intercellular communication in the heart. The use of peptides as pharmaceutical agents has become widely accepted over the past several years and numerous trademarked products are examples of successful peptide-based pharmaceuticals in the US market. In the following paragraphs, we review recent progress and the current status of peptidic sequences that enhance or preserve gap junction function.

\section{Peptide-based strategies to increase or preserve gap junctional communication}

Antiarrhythmic peptide-10 (AAP-10) and its derivatives: In the early 1980s, Aonuma et al. characterized the ability of a series of natural peptides derived from bovine atria to synchronize the beating of cultured chick cardiomyocytes (10). Given that these compounds were shown to have an effect on the synchronization of pacemaker activity of cardiac cells, they were dubbed "anti-arrhythmic peptides" (AAP). A series of followup studies led to the characterization of AAP10, and its d-amino acid analogue ZP123 (later referred to as "rotigaptide") as potential antiarrhythmic agents $(142,755)$. The experiments of Eloff et al. suggested that ZP123 (rotigaptide) may act by preserving intercellular communication under conditions of metabolic stress (168). Separate experiments indicated that this peptide does not modify the morphology of the cardiac action potential; moreover, the peptide was shown to not interact with a panel of integral membrane proteins $(254,321,755)$. Treatment with rotigaptide was shown to increase atrial conduction velocity-with no proarrhythmogenic effect-in animal models of AF and congestive heart failure-related AF. More specifically, in dog models of both atrial ischemia-induced $\mathrm{AF}$ and chronic atrial dilatation, rotigaptide treatment was associated with a significant antiarrhythmic effect. Additionally, it was shown that rotigaptide prevented conduction slowing and reverted established conduction slowing in the isolated rat atrium in a concentration-dependent manner. The effects of rotigaptide were also examined in rabbit atria with chronic atrial dilatation using high-resolution optical mapping on isolated Langendorff perfused rabbit hearts, as well as in cellular studies of gap junctions in ventricular myocytes $(132,168,234,254,318,321,384,627,755)$. Additional animal studies showed that pretreatment with rotigaptide prevented both ventricular and atrial arrhythmias during conditions of acute cardiac ischemia (261) and partially reversed the loss of Cx43 in a canine model of healing infarct (404). Of note, rotigaptide did not prevent conduction slowing or arrhythmia inducibility in this model (404). Rotigaptide was the first drug described as acting primarily by enhancing —or preserving — gap junctional communication (although additional cellular/molecular work would seem justified, and a clear molecular mechanism of action remains lacking). Recent reports have described a new compound (GAP134) as a potential gap junction opener that can be orally administered (518). This compound has been shown to improve conduction and reduce AF/atrial flutter in a canine sterile pericarditis model (571), and to prevent spontaneous ventricular arrhythmias 
and reduce infarct size during myocardial ischemia/reperfusion injury in open chest dogs (262). A modest infarct size reduction was also observed in rats subjected to chronic infarction and treated with rotigaptide (252). Additional promising results in an experimental model of AF in dogs have been reported (361). Future studies will define the efficacy and selectivity of this compound, and its potential use as a therapeutic agent.

While rotigaptide and GAP-134 seem to enhance gap junction communication, their cellular/molecular target is unknown. Attempts to demonstrate binding of rotigaptide to the Cx43 molecule have been unsuccessful. In fact, it is believed that rotigaptide does not exert its effect directly on the connexin molecule, but indirectly via activation of yet unidentified kinases [it is known that rotigaptide modifies the phosphorylation state of Cx43 $(14,318)]$. The latter carries a high risk of connexin-unrelated effects on cell function, as kinases are likely to interact with a variety of substrates, not only $\mathrm{Cx} 43$. The cumulated evidence for rotigaptide-induced effects on Cx43-mediated junctional conductance, and/or Cx43 abundance is substantial see, for example, references 168,253,321,404,627,755, see also reference 132. Dye transfer studies suggest that rotigaptide stimulates Cx43-mediated intercellular communication but it is devoid of activity on $\mathrm{Cx} 26$ and $\mathrm{Cx} 32$, that is, connexins with short C-terminal tail (CT) that lacks several of the regulatory domains of Cx43 and Cx40 (97). Additional data further suggest that connexinregulatory peptides can influence ATP release in cardiac myocytes (98), and improve cell migration rates of human epidermal keratinocytes and dermal fibroblasts (753). Finally, it is interesting to note that an agent that acts by preserving gap junction communication under conditions of stress would, on the other hand, decrease infarct size, given separate evidence indicating that failure of the Cx43 molecule to chemically gate leads to an increase in the size of the infarct (403).

Structure-based peptides to modulate $\mathrm{Cx} 43$ function: aCT-1: A peptide-based strategy, grounded on a structure-activity relation, was also used by Hunter et al. to characterize the interaction of Cx43CT with the scaffolding protein ZO-1 (284). The interaction between these two proteins occurs specifically between the second PDZ domain of ZO-1 and the last nine amino acids of Cx43 [the "PDZbinding domain" (284); see also reference 648]. Accordingly, introduction of a peptide corresponding to the PDZ-binding sequence of Cx43 (dubbed aCT-1) into cells prevented the Cx43-ZO-1 interaction. With this model, the authors demonstrated that prevention of the interaction of $\mathrm{Cx} 43$ with $\mathrm{ZO}-1$ leads to a reduction of peripherally associated $\mathrm{ZO}-1$, accompanied by a significant increase in plaque size. Further analysis indicated that this increased plaque size was not due to increased protein expression or decreased turnover, but rather to unregulated accumulation of gap junctional channels from nonjunctional pools. In a recent study, O'Quinn et al. utilized a myocardial cryoinjury model to demonstrate that local delivery of aCT-1 reduced arrhythmia inducibility, prevented $\mathrm{Cx} 43$ remodeling, and promoted phosphorylation of Cx43 on serine 368 by PKC- $\epsilon$. The authors concluded that aCT-1-mediated increase in Cx43S368 phosphorylation may contribute to reduction in inducible arrhythmia following injury (479). The potential for aCT-1 as a coadjuvant, or as a primary drug in wound healing may extend to other systems. Indeed, Soder and colleagues have demonstrated that of aCT-1 modulates the biological response to silicone implants (640) and can reduce scar progenitors and promote regenerative healing following skinwounding (209). Overall, these results support the notion that the CT domain of Cx43 can be an important target for the development of gap junction-based pharmacology. The results described previously, from the Gourdie laboratory, offer the strongest argument in favor of the notion that $\mathrm{Cx} 43$ regulation (mediated by its CT domain) is a valuable target in the prevention of scar formation, and that agents interfering with the molecular mechanisms of CT-mediated Cx43 regulation can have therapeutic applications not only in the heart, but in other systems as well. Additional studies aimed at developing a Cx43CT-based pharmacology for manipulation of gap junction-mediated intercellular communication, are described later. 
Structure-based peptides to modulate $\mathrm{Cx} 43$ function: RXP-E and its derivatives: An alternative strategy in the search for gap junction modifiers was based on the idea that chemical gating of $\mathrm{Cx} 43$ results from the interaction between the CT domain, acting as a gating particle, and a region of the CL that acts as a receptor $(155,454,606)$. Using phage display, the Delmar laboratory identified a series of peptides containing the sequence "RXP" (arginine, any amino acid, proline) as a consensus $\mathrm{Cx} 43 \mathrm{CT}$ binding motif, and reported that a particular 34-amino acid peptide within this RXP series (dubbed RXP-E) binds to Cx43, prevents heptanol- and low $\mathrm{pH}$-induced gap junction closure, and prevents action potential propagation block $(370,621)$ (Fig. 11).

While those studies showed significant and promising results, further applications of RXP-E as a drug candidate were hampered because of the molecular size and low membrane permeability of this peptide, as well as the metabolic instability and poor oral bioavailability of peptides in general. Peptide mimetics, on the other hand, can be developed to retain the desired biological properties of a peptide. Steps in the design of mimetic molecules include identification of the essential active components (or amino acids) of the peptide sequence (the pharmacophores), determination of their structure/conformation in aqueous solution and finally, development of a corresponding pharmacophore model. Thus, in a separate study, the same laboratory applied a combination of molecular modeling and experimental methods to identify the first group of pharmacophores with Cx43-binding activity (724). Molecular modeling, based on analysis of the RXP series of peptides, led to the identification of both cyclized and linear peptides, 6 to 8 amino acids long, with the ability to bind to the CT of $\mathrm{Cx} 43$ and prevent the closure of $\mathrm{Cx} 43$ channels triggered by octanol superfusion. These data also provided the first three-dimensional imprint of a potential site in the CT region of $\mathrm{Cx} 43$ that could be used for binding of exogenous molecules. Yet, a more precise assignment of the active components of these peptides still remained necessary, to address the question of whether a three-amino acid model can serve as a platform for small molecule design with potential application in living organisms. Thus, a separate study reported a combination of biochemical, spectroscopic, and electrophysiological techniques to demonstrate that the primary sequence $\mathrm{RR}(\mathrm{X}) \mathrm{Y}$ (" $\mathrm{X}$ " being a linker amino acid) is a $\mathrm{Cx} 43$ binding motif. In silico analysis, validated experimentally, further showed that the triangular secondary structure formed by the two arginines and the tyrosine side chains (a "pharmacophore triangle") serves as platform for the design of synthetic molecules that target cardiac gap junction channels. These experiments led to the first peptidomimetic compound capable of preventing chemical gating of Cx43 (compound "ZP2519") (723) and demonstrated that rational, structure-based drug design can be applied to cardiac gap junction pharmacology. These molecules can then serve as tools to determine the role of gap junction regulation in cardiac arrhythmogenesis and, potentially, serve as pharmacological agents for treatment of a selected subset of cardiac arrhythmias.

\section{Final considerations}

Development of a connexin-based pharmacology has made a significant impact in various areas, and perhaps more prominently, in wound healing, as well as in cardiac arrhythmias. As for the latter, at present, pharmacological intervention of cardiac arrhythmias relies, largely, on the use of molecules that impair the function of ion channel proteins. Moreover, while a number of drugs are available to affect sodium, calcium, or potassium currents, gap junctions have eluded the action of exogenous compounds. Whether cardiac gap junctions can be successfully targeted in antiarrhythmic therapy remains unclear. One of the reasons why this question remains unanswered is the lack of compounds that can, selectively and effectively, interfere with gap junction function. The studies described previously seek to develop small molecules that can restore or preserve the function of the major cardiac gap junction channel, $\mathrm{Cx} 43$. In the course of studies from various laboratories, a small arsenal of 
natural and synthetic peptides, as well as pharmacophores, has been generated. Yet, the utility of these molecules in therapeutics remain to be defined. Various authors have proposed that closure of gap junctions during pathologic conditions such as ischemia could lead to malignant ventricular arrhythmias. Yet, loss of coupling under ischemic conditions could also be seen as cardioprotective, as it could preserve the integrity of tissue that is neighbor to an area of damage. Indeed, if gap junctions were to remain open at the ischemic border zone, apoptosis-triggering signals could move through gap junctions, thus extending the area of damage beyond the one that lacks oxygen supply. Studies on brain ischemia have shown that this "bystander effect" may be responsible, at least in part, for the area of "penumbra" that surrounds ischemic tissue (467). (Interestingly, penumbra is not observed in cardiac ischemia and, on the other hand, healing over is well demonstrated.) Opposite to this view is the argument that, while cell-death signals can move through gap junctions, so do molecular signals that can preserve cell integrity, thus helping cell survival within the area at risk. What is important to acknowledge in these contradictory arguments is that our lack of a clear understanding of the beneficial or deleterious effect of gap junction regulation on pathophysiology is, in part, consequent to the lack of experimental tools that can selectively modulate gap junction channels. Much has been learned, for example, from the use of tetrodotoxin to validate the relevance of sodium channels in health and disease. Yet, while the list of pharmacological tools to modulate sodium, potassium, or calcium channels (among others) is extensive, the pharmacology of gap junctions is in its infancy. Future studies are likely to generate more selective and effective gap junction modifiers to assess their importance in medical therapeutics.

\section{Conclusion}

As outlined in this article, our knowledge on the physiology of connexin expression, regulation, and function in both health and disease has increased tremendously over more than three decades of research. However, the article also demonstrates that there is still much to be discovered. The many possible interactions between different connexins and the involvement of an ever increasing list of regulatory binding partners outline the complexity of gap junction physiology. The gap junction proteome may influence both trafficking and function of connexins, and especially the intramolecular rearrangement and binding have emerged as important in the regulation of channel function. Therefore, despite our increasing understanding of the field, several aspects are still subject of debate and contradicting findings remain to be explained.

One of the most heavily debated fields is the role of connexin hemichannels, and although most researchers agree that hemichannels may open under in vitro conditions, the extent to which this happens in vivo remains to be proven. But other surprising noncoupling roles have been established such as the presence of $\mathrm{Cx} 43$ in mitochondria, which regulate mitochondrial function. The potential role of other connexins in mitochondria and/or other intracellular locations, remain an interesting subject of future research.

Many breakthroughs in the gap junction field stem from the development of genetically modified mouse models, where the specific knockout or modification of connexins has allowed us to elucidate their role in different organ systems. Some of these models have confirmed important hypotheses, whereas others have brought up new and unexpected findings and spurred further discoveries. In addition to genetically modified models, the recent progress in the quality of MS studies have increased the possibilities to further explore the chemical modifications that different connexins undergoes during both normal and pathophysiological conditions. Given the important role that posttranslational modification plays in the regulation of connexins, it seems obvious that this is an area, which will also be subject for new interesting findings in the years to come. 
Finally, the involvement of gap junctions in development of various diseases implies the need for further research in this area. Hopefully, basic research regarding the general regulation of gap junctions throughout the different organ systems in the body, as well as further research in pharmacological modulation of gap junctions may bring life saving discoveries in the future. Even though the first pharmacological agent targeting gap junctions remains to find its way to the clinic, the key role of gap junctions in the development of patophysiological conditions such as cardiac arrhythmias, atherosclerosis, cataract, and cancer makes gap junctions a promising pharmacological target.

\section{Acknowledgments}

The authors would like to acknowledge the tremendous work that scientists around the world have put into gap junctional research, and apologize to those of our colleagues, whose important work we did not quote for either lack of space or knowledge. This work was supported by the Danish National Research Foundation (MSN, LNA, and NHHR).

\section{References}

1. Ackert CL, Gittens JE, O’Brien MJ, Eppig JJ, Kidder GM. Intercellular communication via connexin43 gap junctions is required for ovarian folliculogenesis in the mouse. Dev Biol. 2001; 233:258-270. [PubMed: 11336494]

2. Ahmad S, Evans WH. Post-translational integration and oligomerization of connexin 26 in plasma membranes and evidence of formation of membrane pores: Implications for the assembly of gap junctions. Biochem J. 2002; 365:693-699. [PubMed: 11985493]

3. Ai X, Pogwizd SM. Connexin 43 Downregulation and dephosphorylation in nonischemic heart failure is associated with enhanced colocalized protein phosphatase Type 2A. Circ Res. 2005; 96:54-63. [PubMed: 15576650]

4. Ai Z, Fischer A, Spray DC, Brown AM, Fishman GI. Wnt-1 regulation of connexin43 in cardiac myocytes. J Clin Invest. 2000; 105:161-171. [PubMed: 10642594]

5. Al-Ubaidi MR, White TW, Ripps H, Poras I, Avner P, Gomes D, Bruzzone R. Functional properties, developmental regulation, and chromosomal localization of murine connexin 36 , a gapjunctional protein expressed preferentially in retina and brain. J Neurosci Res. 2000; 59:813-826. [PubMed: 10700019]

6. Anderson C, Catoe H, Werner R. MIR-206 regulates connexin43 expression during skeletal muscle development. Nucleic Acids Res. 2006; 34:5863-5871. [PubMed: 17062625]

7. Anderson CM, Bergher JP, Swanson RA. ATP-induced ATP release from astrocytes. J Neurochem. 2004; 88:246-256. [PubMed: 14675168]

8. Anselmi F, Hernandez VH, Crispino G, Seydel A, Ortolano S, Roper SD, Kessaris N, Richardson W, Rickheit G, Filippov MA, Monyer H, Mammano F. ATP release through connexin hemichannels and gap junction transfer of second messengers propagate $\mathrm{Ca} 2+$ signals across the inner ear. Proc Natl Acad Sci U S A. 2008; 105:18770-18775. [PubMed: 19047635]

9. Anumonwo JM, Taffet SM, Gu H, Chanson M, Moreno AP, Delmar M. The carboxyl terminal domain regulates the unitary conductance and voltage dependence of connexin40 gap junction channels. Circ Res. 2001; 88:666-673. [PubMed: 11304488]

10. Aonuma S, Kohama Y, Makino T, Fujisawa Y. Studies of heart. XXI. Amino acid sequence of antiarrhythmic peptide (AAP) isolated from atria. J Pharmacobiodyn. 1982; 5:40-48. [PubMed: 6896215]

11. Armstrong CM, Bezanilla F. Inactivation of sodium channel .2. Gating current experiments. J Gen Physiol. 1977; 70:567-590. [PubMed: 591912]

12. Armstrong SC, Kao R, Gao W, Shivell LC, Downey JM, Honkanen RE, Ganote CE. Comparison of in vitro preconditioning responses of isolated pig and rabbit cardiomyocytes: Effects of a protein phosphatase inhibitor, fostriecin. J Mol Cell Cardiol. 1997; 29:3009-3024. [PubMed: 9405176] 
13. Axelsen LN, Haugan K, Stahlhut M, Kjolbye AL, Hennan JK, Holstein-Rathlou NH, Petersen JS, Nielsen MS. Increasing gap junctional coupling: A tool for dissecting the role of gap junctions. $\mathbf{J}$ Membr Biol. 2007; 216:23-35. [PubMed: 17568971]

14. Axelsen LN, Stahlhut M, Mohammed S, Larsen BD, Nielsen MS, Holstein-Rathlou NH, Andersen S, Jensen ON, Hennan JK, Kjølbye AL. Identification of ischemia-regulated phosphorylation sites in connexin43: A possible target for the antiarrhythmic peptide analogue rotigaptide (ZP123). J Mol Cell Cardiol. 2006; 40:790-798. [PubMed: 16678851]

15. Azarashvili T, Baburina Y, Grachev D, Krestinina O, Evtodienko Y, Stricker R, Reiser G. Calcium-induced permeability transition in rat brain mitochondria is promoted by carbenoxolone through targeting connexin43. Am J Physiol Cell Physiol. 2011; 300:C707-C720. [PubMed: 21148408]

16. Baldo GJ, Gong X, Martinez-Wittinghan FJ, Kumar NM, Gilula NB, Mathias RT. Gap junctional coupling in lenses from alpha(8) connexin knockout mice. J Gen Physiol. 2001; 118:447-456. [PubMed: 11696604]

17. Baldo GJ, Mathias RT. Spatial variations in membrane properties in the intact rat lens. Biophys J. 1992; 63:518-529. [PubMed: 1420894]

18. Balice-Gordon RJ, Bone LJ, Scherer SS. Functional gap junctions in the schwann cell myelin sheath. J Cell Biol. 1998; 142:1095-1104. [PubMed: 9722620]

19. Banerjee D, Das S, Molina SA, Madgwick D, Katz MR, Jena S, Bossmann LK, Pal D, Takemoto DJ. Investigation of the reciprocal relationship between the expression of two gap junction connexin proteins, connexin46 and connexin43. J Biol Chem. 2011

20. Bao L, Locovei S, Dahl G. Pannexin membrane channels are mechanosensitive conduits for ATP. FEBS Lett. 2004; 572:65-68. [PubMed: 15304325]

21. Bao L, Sachs F, Dahl G. Connexins are mechanosensitive. Am J Physiol Cell Physiol. 2004; 287:C1389-C1395. [PubMed: 15475518]

22. Bao X, Reuss L, Altenberg GA. Regulation of purified and reconstituted connexin 43 hemichannels by protein kinase C-mediated phosphorylation of Serine 368. J Biol Chem. 2004; 279:20058-20066. [PubMed: 14973142]

23. Bargiotas P, Monyer H, Schwaninger M. Hemichannels in cerebral ischemia. Curr Mol Med. 2009; 9:186-194. [PubMed: 19275626]

24. Barrio LC, Capel J, Jarillo JA, Castro C, Revilla A. Species-specific voltage-gating properties of connexin-45 junctions expressed in Xenopus oocytes. Biophys J. 1997; 73:757-769. [PubMed: 9251792]

25. Barrio LC, Suchyna T, Bargiello T, Xu LX, Roginski RS, Bennett MV, Nicholson BJ. Gap junctions formed by connexins 26 and 32 alone and in combination are differently affected by applied voltage. Proc Natl Acad Sci U S A. 1991; 88:8410-8414. [PubMed: 1717979]

26. Bassnett S, Duncan G. Direct measurement of $\mathrm{pH}$ in the rat lens by ion-sensitive microelectrodes. Exp Eye Res. 1985; 40:585-590. [PubMed: 4007073]

27. Bastiaanse EM, Jongsma HJ, van der Laarse A, Takens-Kwak BR. Heptanol-induced decrease in cardiac gap junctional conductance is mediated by a decrease in the fluidity of membranous cholesterol-rich domains. J Membr Biol. 1993; 136:135-145. [PubMed: 7508980]

28. Batias C, Siffroi JP, Fenichel P, Pointis G, Segretain D. Connexin43 gene expression and regulation in the rodent seminiferous epithelium. J Histochem Cytochem. 2000; 48:793-805. [PubMed: 10820153]

29. Bavamian S, Klee P, Britan A, Populaire C, Caille D, Cancela J, Charollais A, Meda P. Islet-cellto-cell communication as basis for normal insulin secretion. Diabetes Obes Metab. 2007; 9(Suppl 2):118-132. [PubMed: 17919186]

30. Beardslee MA, Laing JG, Beyer EC, Saffitz JE. Rapid turnover of connexin43 in the adult rat heart. Circ Res. 1998; 83:629-635. [PubMed: 9742058]

31. Beardslee MA, Lerner DL, Tadros PN, Laing JG, Beyer EC, Yamada KA, Kléber AG, Schuessler RB, Saffitz JE. Dephosphorylation and intracellular redistribution of ventricular connexin43 during electrical uncoupling induced by ischemia. Circ Res. 2000; 87:656-662. [PubMed: 11029400] 
32. Beblo DA, Veenstra RD. Monovalent cation permeation through the connexin40 gap junction channel. Cs, Rb, K, Na, Li, TEA, TMA, TBA, and effects of anions $\mathrm{Br}, \mathrm{Cl}, \mathrm{F}$, acetate, aspartate, glutamate, and NO3. J Gen Physiol. 1997; 109:509-522. [PubMed: 9101408]

33. Beblo DA, Wang HZ, Beyer EC, Westphale EM, Veenstra RD. Unique conductance, gating, and selective permeability properties of gap junction channels formed by connexin40. Circ Res. 1995; 77:813-822. [PubMed: 7554128]

34. Belluardo N, Trovato-Salinaro A, Mudo G, Hurd YL, Condorelli DF. Structure, chromosomal localization, and brain expression of human Cx36 gene. J Neurosci Res. 1999; 57:740-752. [PubMed: 10462698]

35. Beltramello M, Piazza V, Bukauskas FF, Pozzan T, Mammano F. Impaired permeability to Ins(1,4,5)P3 in a mutant connexin underlies recessive hereditary deafness. Nat Cell Biol. 2005; 7:63-69. [PubMed: 15592461]

36. Belzer V, Kobilo T, Rich A, Hanani M. Intercellular coupling among interstitial cells of Cajal in the guinea pig small intestine. Cell Tissue Res. 2002; 307:15-21. [PubMed: 11810310]

37. Belzer V, Nissan A, Freund HR, Hanani M. Coupling among interstitial cells of Cajal in the human ileum. Neurogastroenterol Motil. 2004; 16:75-80. [PubMed: 14764207]

38. Bennett MV, Contreras JE, Bukauskas FF, Saez JC. New roles for astrocytes: Gap junction hemichannels have something to communicate. Trends Neurosci. 2003; 26:610-617. [PubMed: 14585601]

39. Bermudez-Fajardo A, Yliharsila M, Evans WH, Newby AC, Oviedo-Orta E. CD4+ T lymphocyte subsets express connexin 43 and establish gap junction channel communication with macrophages in vitro. J Leukoc Biol. 2007; 82:608-612. [PubMed: 17596336]

40. Berthoud VM, Beyer EC, Kurata WE, Lau AF, Lampe PD. The Gapjunction protein Connexin 56 is phosphorylated in the intracellular loop and the carboxy-terminal region. Eur J Biochem. 1997; 244:89-97. [PubMed: 9063450]

41. Bevans CG, Harris AL. Regulation of connexin channels by pH. Direct action of the protonated form of taurine and other aminosulfonates. J Biol Chem. 1999; 274:3711-3719. [PubMed: 9920923]

42. Bevilacqua LM, Simon AM, Maguire CT, Gehrmann J, Wakimoto H, Paul DL, Berul CI. A targeted disruption in connexin40 leads to distinct atrioventricular conduction defects. J Interv Card Electrophysiol. 2000; 4:459-467. [PubMed: 11046183]

43. Beyer EC, Kistler J, Paul DL, Goodenough DA. Antisera directed against connexin 43 peptides react with a $43-\mathrm{kD}$ protein localized to gap junctions in myocardium and other tissues. J Cell Biol. 1989; 108:595-605. [PubMed: 2537319]

44. Bierhuizen MF, Boulaksil M, van Stuijvenberg L, van der Nagel R, Jansen AT, Mutsaers NA, Yildirim C, van Veen TA, de Windt LJ, Vos MA, van Rijen HV. In calcineurin-induced cardiac hypertrophy expression of Nav1.5, Cx40 and Cx43 is reduced by different mechanisms. J Mol Cell Cardiol. 2008; 45:373-384. [PubMed: 18662696]

45. Bierhuizen MF, van Amersfoorth SC, Groenewegen WA, Vliex S, Jongsma HJ. Characterization of the rat connexin40 promoter: Two Sp1/Sp3 binding sites contribute to transcriptional activation. Cardiovasc Res. 2000; 46:511-522. [PubMed: 10912461]

46. Blackburn JP, Peters NS, Yeh HI, Rothery S, Green CR, Severs NJ. Upregulation of connexin43 gap junctions during early stages of human coronary atherosclerosis. Arterioscler Thromb Vasc Biol. 1995; 15:1219-1228. [PubMed: 7627716]

47. Bloomfield SA, Volgyi B. The diverse functional roles and regulation of neuronal gap junctions in the retina. Nat Rev Neurosci. 2009; 10:495-506. [PubMed: 19491906]

48. Boassa D, Solan JL, Papas A, Thornton P, Lampe PD, Sosinsky GE. Trafficking and recycling of the connexin43 gap junction protein during mitosis. Traffic. 2010; 11:1471-1486. [PubMed: 20716111]

49. Boengler K, Dodoni G, Rodriguez-Sinovas A, Cabestrero A, Ruiz-Meana M, Gres P, Konietzka I, Lopez-Iglesias C, Garcia-Dorado D, Di LF, Heusch G, Schulz R. Connexin 43 in cardiomyocyte mitochondria and its increase by ischemic preconditioning. Cardiovasc Res. 2005; 67:234-244. [PubMed: 15919068] 
50. Boengler K, Konietzka I, Buechert A, Heinen Y, Garcia-Dorado D, Heusch G, Schulz R. Loss of ischemic preconditioning's cardioprotection in aged mouse hearts is associated with reduced gap junctional and mitochondrial levels of connexin 43. Am J Physiol Heart Circ Physiol. 2007; 292:H1764-H1769. [PubMed: 17142336]

51. Boengler K, Stahlhofen S, van de Sand A, Gres P, Ruiz-Meana M, Garcia-Dorado D, Heusch G, Schulz R. Presence of connexin 43 in subsarcolemmal, but not in interfibrillar cardiomyocyte mitochondria. Basic Res Cardiol. 2009; 104:141-147. [PubMed: 19242638]

52. Bolon ML, Peng T, Kidder GM, Tyml K. Lipopolysaccharide plus hypoxia and reoxygenation synergistically reduce electrical coupling between microvascular endothelial cells by dephosphorylating Connexin40. J Cell Physiol. 2008; 217:350-359. [PubMed: 18521823]

53. Bond SL, Bechberger JF, Khoo NK, Naus CC. Transfection of C6 glioma cells with connexin32: The effects of expression of a nonendogenous gap junction protein. Cell Growth Differ. 1994; 5:179-186. [PubMed: 8180131]

54. Bondurand N, Girard M, Pingault V, Lemort N, Dubourg O, Goossens M. Human Connexin 32, a gap junction protein altered in the X-linked form of Charcot-Marie-Tooth disease, is directly regulated by the transcription factor SOX10. Hum Mol Genet. 2001; 10:2783-2795. [PubMed: 11734543]

55. Boogerd KJ, Wong LY, Christoffels VM, Klarenbeek M, Ruijter JM, Moorman AF, Barnett P. Msx1 and Msx2 are functional interacting partners of T-box factors in the regulation of Connexin43. Cardiovasc Res. 2008; 78:485-493. [PubMed: 18285513]

56. Borinstein SC, Conerly M, Dzieciatkowski S, Biswas S, Washington MK, Trobridge P, Henikoff S, Grady WM. Aberrant DNA methylation occurs in colon neoplasms arising in the azoxymethane colon cancer model. Mol Carcinog. 2010; 49:94-103. [PubMed: 19777566]

57. Bouvier D, Spagnol G, Chenavas S, Kieken F, Vitrac H, Brownell S, Kellezi A, Forge V, Sorgen PL. Characterization of the structure and intermolecular interactions between the connexin 40 and connexin43 carboxyl-terminal and cytoplasmic loop domains. J Biol Chem. 2009; 284:3425734271. [PubMed: 19808665]

58. Boyett MR, Inada S, Yoo S, Li J, Liu J, Tellez J, Greener ID, Honjo H, Billeter R, Lei M, Zhang H, Efimov IR, Dobrzynski H. Connexins in the sinoatrial and atrioventricular nodes. Adv Cardiol. 2006; 42:175-197. [PubMed: 16646591]

59. Braet K, Aspeslagh S, Vandamme W, Willecke K, Martin PE, Evans WH, Leybaert L. Pharmacological sensitivity of ATP release triggered by photoliberation of inositol-1,4,5trisphosphate and zero extracellular calcium in brain endothelial cells. J Cell Physiol. 2003; 197:205-213. [PubMed: 14502560]

60. Braet K, Vandamme W, Martin PE, Evans WH, Leybaert L. Photoliberating inositol-1,4,5trisphosphate triggers ATP release that is blocked by the connexin mimetic peptide gap 26. Cell Calcium. 2003; 33:37-48. [PubMed: 12526886]

61. Braun AP, Schulman H. The Multifunctional calcium/calmodulindependent protein kinase: From form to ction. Annu Rev Physiol. 1995; 57:417-445. [PubMed: 7778873]

62. Bravo-Moreno JF, Diaz-Sanchez V, Montoya-Flores JG, Lamoyi E, Saez JC, Perez-Armendariz EM. Expression of connexin43 in mouse Leydig, Sertoli, and germinal cells at different stages of postnatal development. Anat Rec. 2001; 264:13-24. [PubMed: 11505367]

63. Brehm R, Zeiler M, Ruttinger C, Herde K, Kibschull M, Winterhager E, Willecke K, Guillou F, Lecureuil C, Steger K, Konrad L, Biermann K, Failing K, Bergmann M. A sertoli cell-specific knockout of connexin43 prevents initiation of spermatogenesis. Am J Pathol. 2007; 171:19-31. [PubMed: 17591950]

64. Brink PR, Cronin K, Banach K, Peterson E, Westphale EM, Seul KH, Ramanan SV, Beyer EC. Evidence for heteromeric gap junction channels formed from rat connexin43 and human connexin37. Am J Physiol. 1997; 273:C1386-C1396. [PubMed: 9357785]

65. Bruneau BG, Nemer G, Schmitt JP, Charron F, Robitaille L, Caron S, Conner DA, Gessler M, Nemer M, Seidman CE, Seidman JG. A murine model of Holt-Oram syndrome defines roles of the T-box transcription factor Tbx5 in cardiogenesis and disease. Cell. 2001; 106:709-721. [PubMed: 11572777] 
66. Bruzzone R, Hormuzdi SG, Barbe MT, Herb A, Monyer H. Pannexins, a family of gap junction proteins expressed in brain. Proc Natl Acad Sci U S A. 2003; 100:13644-13649. [PubMed: 14597722]

67. Bruzzone S, Franco L, Guida L, Zocchi E, Contini P, Bisso A, Usai C, De FA. A self-restricted CD38-connexin 43 cross-talk affects NAD+ and cyclic ADP-ribose metabolism and regulates intracellular calcium in 3T3 fibroblasts. J Biol Chem. 2001; 276:48300-48308. [PubMed: 11602597]

68. Bruzzone S, Guida L, Zocchi E, Franco L, De FA. Connexin 43 hemi channels mediate Ca2+regulated transmembrane NAD+ fluxes in intact cells. FASEB J. 2001; 15:10-12. [PubMed: 11099492]

69. Buhl DL, Harris KD, Hormuzdi SG, Monyer H, Buzsaki G. Selective impairment of hippocampal gamma oscillations in connexin-36 knockout mouse in vivo. J Neurosci. 2003; 23:1013-1018. [PubMed: 12574431]

70. Bukauskas FF, Bukauskiene A, Bennett MV, Verselis VK. Gating properties of gap junction channels assembled from connexin43 and connexin43 fused with green fluorescent protein. Biophys J. 2001; 81:137-152. [PubMed: 11423402]

71. Bukauskas FF, Elfgang C, Willecke K, Weingart R. Biophysical properties of gap junction channels formed by mouse connexin40 in induced pairs of transfected human HeLa cells. Biophys J. 1995; 68:2289-2298. [PubMed: 7544165]

72. Bukauskas FF, Verselis VK. Gap junction channel gating. Biochim Biophys Acta. 2004; 1662:4260. [PubMed: 15033578]

73. Bukauskas FF, Weingart R. Multiple conductance states of newly formed single gap junction channels between insect cells. Pflugers Arch. 1993; 423:152-154. [PubMed: 7683788]

74. Bukauskas FF, Weingart R. Voltage-dependent gating of single gap junction channels in an insect cell line. Biophys J. 1994; 67:613-625. [PubMed: 7524710]

75. Burr GS, Mitchell CK, Keflemariam YJ, Heidelberger R, O’Brien J. Calcium-dependent binding of calmodulin to neuronal gap junction proteins. Biochem Biophys Res Commun. 2005; 335:11911198. [PubMed: 16112650]

76. Burt JM. Block of intercellular communication: Interaction of intracellular $\mathrm{H}+$ and $\mathrm{Ca} 2+\mathrm{Am} \mathrm{J}$ Physiol. 1987; 253:C607-C612. [PubMed: 2444111]

77. Burt JM, Massey KD, Minnich BN. Uncoupling of cardiac cells by fatty acids: Structure-activity relationships. Am J Physiol. 1991; 260:C439-C448. [PubMed: 2003571]

78. Burt JM, Spray DC. Volatile anesthetics block intercellular communication between neonatal rat myocardial cells. Circ Res. 1989; 65:829-837. [PubMed: 2766493]

79. Butterweck A, Gergs U, Elfgang C, Willecke K, Traub O. Immunochemical characterization of the gap junction protein connexin45 in mouse kidney and transfected human HeLa cells. J Membr Biol. 1994; 141:247-256. [PubMed: 7807524]

80. Cameron SJ, Malik S, Akaike M, Lerner-Marmarosh N, Yan C, Lee JD, Abe JI, Yang J. Regulation of epidermal growth factor-induced Connexin 43 gap junction communication by big mitogen-activated protein kinase 1/ERK5 but not ERK1/2 kinase activation. J Biol Chem. 2003; 278:18682-18688. [PubMed: 12637502]

81. Campos-Toimil M, Edwardson JM, Thomas P. Acetylcholine-induced zymogen granule exocytosis: Comparison between acini and single pancreatic acinar cells. Pancreas. 2002; 24:179183. [PubMed: 11854623]

82. Cancelas JA, Koevoet WL, De Koning AE, Mayen AE, Rombouts EJ, Ploemacher RE. Connexin-43 gap junctions are involved in multiconnexin-expressing stromal support of hemopoietic progenitors and stem cells. Blood. 2000; 96:498-505. [PubMed: 10887111]

83. Chadjichristos CE, Scheckenbach KE, van Veen TA, Richani Sarieddine MZ, de WC, Yang Z, Roth I, Bacchetta M, Viswambharan H, Foglia B, Dudez T, van Kempen MJ, Coenjaerts FE, Miquerol L, Deutsch U, Jongsma HJ, Chanson M, Kwak BR. Endothelial-specific deletion of connexin40 promotes atherosclerosis by increasing CD73-dependent leukocyte adhesion. Circulation. 2010; 121:123-131. [PubMed: 20026782]

84. Chaldoupi SM, Loh P, Hauer RNW, de Bakker JMT, van Rijen HVM. The role of connexin40 in atrial fibrillation. Cardiovasc Res. 2009; 84:15-23. [PubMed: 19535379] 
85. Chappell RL, Qian H, Zakevicius J, Ripps H. Histidine suppresses zinc modulation of connexin hemichannels. Biol Bull. 2004; 207:188-190. [PubMed: 15616348]

86. Chappell RL, Zakevicius J, Ripps H. Zinc modulation of hemichannel currents in Xenopus oocytes. Biol Bull. 2003; 205:209-211. [PubMed: 14583533]

87. Chen JT, Cheng YW, Chou MC, Sen-Lin T, Lai WW, Ho WL, Lee H. The correlation between aberrant connexin 43 mRNA expression induced by promoter methylation and nodal micrometastasis in nonsmall cell lung cancer. Clin Cancer Res. 2003; 9:4200-4204. [PubMed: 14519646]

88. Chen Y, Huhn D, Knosel T, Pacyna-Gengelbach M, Deutschmann N, Petersen I. Downregulation of connexin 26 in human lung cancer is related to promoter methylation. Int J Cancer. 2005; 113:14-21. [PubMed: 15386363]

89. Chow L, Lye SJ. Expression of the gap junction protein connexin-43 is increased in the human myometrium toward term and with the onset of labor. Am J Obstet Gynecol. 1994; 170:788-795. [PubMed: 8141203]

90. Christoffels VM, Hoogaars WM, Tessari A, Clout DE, Moorman AF, Campione M. T-box transcription factor Tbx2 represses differentiation and formation of the cardiac chambers. Dev Dyn. 2004; 229:763-770. [PubMed: 15042700]

91. Chu G, Carr AN, Young KB, Lester JW, Yatani A, Sanbe A, Colbert MC, Schwartz SM, Frank KF, Lampe PD, Robbins J, Molkentin JD, Kranias EG. Enhanced myocyte contractility and Ca2+ handling in a calcineurin transgenic model of heart failure. Cardiovasc Res. 2002; 54:105-116. [PubMed: 12062367]

92. Chung SS, Choi C, Wang X, Hallock L, Wolgemuth DJ. Aberrant distribution of junctional complex components in retinoic acid receptor alpha-deficient mice. Microsc Res Tech. 2010; 73:583-596. [PubMed: 19937743]

93. Chung TH, Wang SM, Chang YC, Chen YL, Wu JC. 18+|-glycyrrhetinic acid promotes src interaction with connexin43 in rat cardiomyocytes. J Cell Biochem. 2007; 100:653-664. [PubMed: 16983688]

94. Cina C, Maass K, Theis M, Willecke K, Bechberger JF, Naus CC. Involvement of the cytoplasmic C-terminal domain of connexin43 in neuronal migration. J Neurosci. 2009; 29:2009-2021. [PubMed: 19228955]

95. Ciray HN, Guner H, Hakansson H, Tekelioglu M, Roomans GM, Ulmsten U. Morphometric analysis of gap junctions in nonpregnant and term pregnant human myometrium. Acta Obstet Gynecol Scand. 1995; 74:497-504. [PubMed: 7618446]

96. Clair C, Chalumeau C, Tordjmann T, Poggioli J, Erneux C, Dupont G, Combettes L. Investigation of the roles of $\mathrm{Ca}(2+)$ and $\mathrm{InsP}(3)$ diffusion in the coordination of $\mathrm{Ca}(2+)$ signals between connected hepatocytes. J Cell Sci. 2001; 114:1999-2007. [PubMed: 11493636]

97. Clarke TC, Thomas D, Petersen JS, Evans WH, Martin PE. The antiarrhythmic peptide rotigaptide (ZP123) increases gap junction intercellular communication in cardiac myocytes and HeLa cells expressing connexin 43. Br J Pharmacol. 2006; 147:486-495. [PubMed: 16415913]

98. Clarke TC, Williams OJ, Martin PE, Evans WH. ATP release by cardiac myocytes in a simulated ischaemia model: Inhibition by a connexin mimetic and enhancement by an antiarrhythmic peptide. Eur J Pharmacol. 2009; 605:9-14. [PubMed: 19101539]

99. Cluff AH, Bystrom B, Klimaviciute A, Dahlqvist C, Cebers G, Malmstrom A, Ekman-Ordeberg G. Prolonged labour associated with lower expression of syndecan 3 and connexin 43 in human uterine tissue. Reprod Biol Endocrinol. 2006; 4:24. [PubMed: 16674815]

100. Coco S, Calegari F, Pravettoni E, Pozzi D, Taverna E, Rosa P, Matteoli M, Verderio C. Storage and release of ATP from astrocytes in culture. J Biol Chem. 2003; 278:1354-1362. [PubMed: 12414798]

101. Cohen PTW. Novel protein serine/threonine phosphatases: Variety is the spice of life. Trends Biochem Sci. 1997; 22:245-251. [PubMed: 9255065]

102. Colussi C, Rosati J, Straino S, Spallotta F, Berni R, Stilli D, Rossi S, Musso E, Macchi E, Mai A, Sbardella G, Castellano S, Chimenti C, Frustaci A, Nebbioso A, Altucci L, Capogrossi MC, Gaetano C. Ne-lysine acetylation determines dissociation from GAP junctions and lateralization 
of connexin 43 in normal and dystrophic heart. Proc Natl Acad Sci U S A. 2011; 108:2795-2800. [PubMed: 21282606]

103. Condorelli DF, Belluardo N, Trovato-Salinaro A, Mudo G. Expression of Cx36 in mammalian neurons. Brain Res Brain Res Rev. 2000; 32:72-85. [PubMed: 10751658]

104. Connors BW, Long MA. Electrical synapses in the mammalian brain. Annu Rev Neurosci. 2004; 27:393-418. [PubMed: 15217338]

105. Connors BW, Ransom BR, Kunis DM, Gutnick MJ. Activity-dependent K+ accumulation in the developing rat optic nerve. Science. 1982; 216:1341-1343. [PubMed: 7079771]

106. Contreras JE, Saez JC, Bukauskas FF, Bennett MV. Gating and regulation of connexin 43 (Cx43) hemichannels. Proc Natl Acad Sci U S A. 2003; 100:11388-11393. [PubMed: 13130072]

107. Contreras JE, Sanchez HA, Eugenin EA, Speidel D, Theis M, Willecke K, Bukauskas FF, Bennett MV, Saez JC. Metabolic inhibition induces opening of unapposed connexin 43 gap junction hemichannels and reduces gap junctional communication in cortical astrocytes in culture. Proc Natl Acad Sci U S A. 2002; 99:495-500. [PubMed: 11756680]

108. Contreras JE, Sanchez HA, Veliz LP, Bukauskas FF, Bennett MV, Saez JC. Role of connexinbased gap junction channels and hemichannels in ischemia-induced cell death in nervous tissue. Brain Res Brain Res Rev. 2004; 47:290-303. [PubMed: 15572178]

109. Cooper CD, Lampe PD. Casein Kinase 1 Regulates Connexin-43 Gap Junction Assembly. J Biol Chem. 2002; 277:44962-44968. [PubMed: 12270943]

110. Cox NJ, Frigge M, Nicolae DL, Concannon P, Hanis CL, Bell GI, Kong A. Loci on chromosomes 2 (NIDDM1) and 15 interact to increase susceptibility to diabetes in Mexican Americans. Nat Genet. 1999; 21:213-215. [PubMed: 9988276]

111. Cristancho JM, Campos de Carvalho AC, Varanda WA. Short term regulation of cell-cell communication in TM3 Leydig cells.Aperforated patch study. Biochim Biophys Acta. 2000; 1496:325-332. [PubMed: 10771100]

112. Cronier L, Crespin S, Strale PO, Defamie N, Mesnil M. Gap junctions and cancer: New functions for an old story. Antioxid Redox Signal. 2009; 11:323-338. [PubMed: 18834328]

113. Dahl G. Gap junction-mimetic peptides do work, but in unexpected ways. Cell Commun Adhes. 2007; 14:259-264. [PubMed: 18392993]

114. Dahl G, Locovei S. Pannexin: To gap or not to gap, is that a question? IUBMB Life. 2006; 58:409-419. [PubMed: 16801216]

115. Dahl G, Nonner W, Werner R. Attempts to define functional domains of gap junction proteins with synthetic peptides. Biophys J. 1994; 67:1816-1822. [PubMed: 7858120]

116. Dahm R, van MJ, Prescott AR, Quinlan RA. Gap junctions containing alpha8-connexin (MP70) in the adult mammalian lens epithelium suggests a re-evaluation of its role in the lens. Exp Eye Res. 1999; 69:45-56. [PubMed: 10375448]

117. Dang X, Doble BW, Kardami E. The carboxy-tail of connexin-43 localizes to the nucleus and inhibits cell growth. Mol Cell Biochem. 2003; 242:35-38. [PubMed: 12619863]

118. Dang X, Jeyaraman M, Kardami E. Regulation of connexin-43-mediated growth inhibition by a phosphorylatable amino-acid is independent of gap junction-forming ability. Mol Cell Biochem. 2006; 289:201-207. [PubMed: 16718370]

119. Daniel EE. Communication between interstitial cells of Cajal and gastrointestinal muscle. Neurogastroenterol Motil. 2004; 16(Suppl 1):118-122. [PubMed: 15066016]

120. Daniel EE, Wang YF. Gap junctions in intestinal smooth muscle and interstitial cells of Cajal. Microsc Res Tech. 1999; 47:309-320. [PubMed: 10602290]

121. Danik SB, Liu F, Zhang J, Suk HJ, Morley GE, Fishman GI, Gutstein DE. Modulation of cardiac gap junction expression and arrhythmic susceptibility. Circ Res. 2004; 95:1035-1041. [PubMed: 15499029]

122. Darrow BJ, Laing JG, Lampe PD, Saffitz JE, Beyer EC. Expression of multiple connexins in cultured neonatal rat ventricular myocytes. Circ Res. 1995; 76:381-387. [PubMed: 7859384]

123. Das S, Smith TD, Das Sarma J, Ritzenthaler JD, Maza J, Kaplan BE, Cunningham LA, Suaud L, Hubbard MJ, Rubenstein RC, Koval M. ERp29 restricts Connexin43 oligomerization in the endoplasmic reticulum. Mol Biol Cell. 2009; 20:2593-2604. [PubMed: 19321666] 
124. Dasgupta C, Martinez AM, Zuppan CW, Shah MM, Bailey LL, Fletcher WH. Identification of connexin43 (alpha1) gap junction gene mutations in patients with hypoplastic left heart syndrome by denaturing gradient gel electrophoresis (DGGE). Mutat Res. 2001; 479:173-186. [PubMed: 11470490]

125. Davidson JS, Baumgarten IM. Glycyrrhetinic acid derivatives: A novel class of inhibitors of gapjunctional intercellular communication. Structure-activity relationships. J Pharmacol Exp Ther. 1988; 246:1104-1107. [PubMed: 3418512]

126. Davidson JS, Baumgarten IM, Harley EH. Reversible inhibition of intercellular junctional communication by glycyrrhetinic acid. Biochem Biophys Res Commun. 1986; 134:29-36. [PubMed: 3947327]

127. Davis LM, Kanter HL, Beyer EC, Saffitz JE. Distinct gap junction protein phenotypes in cardiac tissues with disparate conduction properties. J Am Coll Cardiol. 1994; 24:1124-1132. [PubMed: 7930207]

128. Davis LM, Rodefeld ME, Green K, Beyer EC, Saffitz JE. Gap junction protein phenotypes of the human heart and conduction system. J Cardiovasc Electrophysiol. 1995; 6:813-822. [PubMed: 8542077]

129. De Maio A, Gingalewski C, Theodorakis NG, Clemens MG. Interruption of hepatic gap junctional communication in the rat during inflammation induced by bacterial lipopolysaccharide. Shock. 2000; 14:53-59. [PubMed: 10909894]

130. De Mello WC. Influence of the sodium pump on intercellular communication in heart fibres: Effect of intracellular injection of sodium ion on electrical coupling. J Physiol. 1976; 263:171197. [PubMed: 1018231]

131. de Pina-Benabou MH, Szostak V, Kyrozis A, Rempe D, Uziel D, Urban-Maldonado M, Benabou S, Spray DC, Federoff HJ, Stanton PK, Rozental R. Blockade of gap junctions in vivo provides neuroprotection after perinatal global ischemia. Stroke. 2005; 36:2232-2237. [PubMed: 16179575]

132. De Vuyst E, Boengler K, Antoons G, Sipido KR, Schulz R, Leybaert L. Pharmacological modulation of connexin-formed channels in cardiac pathophysiology. Br J Pharmacol. 2011; 163:469-483. [PubMed: 21265827]

133. de Wit C, Griffith TM. Connexins and gap junctions in the EDHF phenomenon and conducted vasomotor responses. Pflugers Arch. 2010; 459:897-914. [PubMed: 20379740]

134. deWit C, Roos F, Bolz SS, Pohl U. Lack of vascular connexin 40 is associated with hypertension and irregular arteriolar vasomotion. Physiol Genomics. 2003; 13:169-177. [PubMed: 12700362]

135. De Pinto V, Messina A, Lane DJ, Lawen A. Voltage-dependent anionselective channel (VDAC) in the plasma membrane. FEBS Lett. 2010; 584:1793-1799. [PubMed: 20184885]

136. Deans MR, Gibson JR, Sellitto C, Connors BW, Paul DL. Synchronous activity of inhibitory networks in neocortex requires electrical synapses containing connexin36. Neuron. 2001; 31:477-485. [PubMed: 11516403]

137. Decrouy X, Gasc JM, Pointis G, Segretain D. Functional characterization of Cx43 based gap junctions during spermatogenesis. J Cell Physiol. 2004; 200:146-154. [PubMed: 15137067]

138. Delmar M, Coombs W, Sorgen P, Duffy HS, Taffet SM. Structural bases for the chemical regulation of Connexin43 channels. Cardiovasc Res. 2004; 62:268-275. [PubMed: 15094347]

139. Delorme B, Dahl E, Jarry-Guichard T, Marics I, Briand JP, Willecke K, Gros D, TheveniauRuissy M. Developmental regulation of connexin 40 gene expression in mouse heart correlates with the differentiation of the conduction system. Dev Dyn. 1995; 204:358-371. [PubMed: 8601030]

140. DeRosa AM, Mui R, Srinivas M, White TW. Functional characterization of a naturally occurring Cx50 truncation. Invest Ophthalmol Vis Sci. 2006; 47:4474-4481. [PubMed: 17003442]

141. DeVries SH, Schwartz EA. Hemi-gap-junction channels in solitary horizontal cells of the catfish retina. J Physiol. 1992; 445:201-230. [PubMed: 1380084]

142. Dhein S, Manicone N, Muller A, Gerwin R, Ziskoven U, Irankhahi A, Minke C, Klaus W. A new synthetic antiarrhythmic peptide reduces dispersion of epicardial activation recovery interval and diminishes alterations of epicardial activation patterns induced by regional ischemia. A mapping study. Naunyn Schmiedebergs Arch Pharmacol. 1994; 350:174-184. [PubMed: 7990974] 
143. Di VF, Chiozzi P, Falzoni S, Ferrari D, Sanz JM, Venketaraman V, Baricordi OR. Cytolytic P2X purinoceptors. Cell Death Differ. 1998; 5:191-199. [PubMed: 10200464]

144. Di WL, Rugg EL, Leigh IM, Kelsell DP. Multiple epidermal connexins are expressed in different keratinocyte subpopulations including connexin 31. J Invest Dermatol. 2001; 117:958-964. [PubMed: 11676838]

145. Diez JA, Ahmad S, Evans WH. Assembly of heteromeric connexons in guinea-pig liver en route to the Golgi apparatus, plasma membrane and gap junctions. Eur J Biochem. 1999; 262:142-148. [PubMed: 10231375]

146. Díez JA, Elvira M, Villalobo A. The epidermal growth factor receptor tyrosine kinase phosphorylates connexin32. Mol Cell Biochem. 1998; 187:201-210. [PubMed: 9788758]

147. Dixon DB, Takahashi K, Bieda M, Copenhagen DR. Quinine, intracellular pH and modulation of hemi-gap junctions in catfish horizontal cells. Vision Res. 1996; 36:3925-3931. [PubMed: 9068845]

148. Doble BW, Dang X, Ping P, Fandrich RR, Nickel BE, Jin Y, Cattini PA, Kardami E. Phosphorylation of serine 262 in the gap junction protein connexin-43 regulates DNA synthesis in cell-cell contact forming cardiomyocytes. J Cell Sci. 2004; 117:507-514. [PubMed: 14702389]

149. Dobrowolski R, Willecke K. Connexin-caused genetic diseases and corresponding mouse models. Antioxid Redox Signal. 2009; 11:283-295. [PubMed: 18831677]

150. Dodd R, Peracchia C, Stolady D, T+Âr $+\hat{A} k$ K. Calmodulin association with Connexin32-derived peptides suggests trans-domain interaction in chemical gating of gap junction channels. J Biol Chem. 2008; 283:26911-26920. [PubMed: 18676375]

151. Dong X, Yu C, Shynlova O, Challis JR, Rennie PS, Lye SJ. p54nrb is a transcriptional corepressor of the progesterone receptor that modulates transcription of the labor-associated gene, connexin 43 (Gja1). Mol Endocrinol. 2009; 23:1147-1160. [PubMed: 19423654]

152. Doring B, Shynlova O, Tsui P, Eckardt D, Janssen-Bienhold U, Hofmann F, Feil S, Feil R, Lye SJ, Willecke K. Ablation of connexin43 in uterine smooth muscle cells of the mouse causes delayed parturition. J Cell Sci. 2006; 119:1715-1722. [PubMed: 16595547]

153. Duffy HS, Delmar M, Spray DC. Formation of the gap junction nexus: Binding partners for connexins. J Physiol Paris. 2002; 96:243-249. [PubMed: 12445902]

154. Duffy HS, Iacobas I, Hotchkiss K, Hirst-Jensen BJ, Bosco A, Dandachi N, Dermietzel R, Sorgen PL, Spray DC. The gap junction protein connexin32 interacts with the Src homology 3/hook domain of discs large homolog 1. J Biol Chem. 2007; 282:9789-9796. [PubMed: 17284442]

155. Duffy HS, Sorgen PL, Girvin ME, O’Donnell P, Coombs W, Taffet SM, Delmar M, Spray DC. $\mathrm{pH}$-dependent intramolecular binding and structure involving $\mathrm{Cx} 43$ cytoplasmic domains. J Biol Chem. 2002; 277:36706-36714. [PubMed: 12151412]

156. Dupont E, Matsushita T, Kaba RA, Vozzi C, Coppen SR, Khan N, Kaprielian R, Yacoub MH, Severs NJ. Altered connexin expression in human congestive heart failure. J Mol Cell Cardiol. 2001; 33:359-371. [PubMed: 11162139]

157. Dupont G, Tordjmann T, Clair C, Swillens S, Claret M, Combettes L. Mechanism of receptororiented intercellular calcium wave propagation in hepatocytes. FASEB J. 2000; 14:279-289. [PubMed: 10657984]

158. Duthe F, Plaisance I, Sarrouilhe D, Herv $+\circledR$ JC. Endogenous protein phosphatase 1 runs down gap junctional communication of rat ventricular myocytes. Am J Physiol Cell Physiol. 2001; 281:C1648-C1656. [PubMed: 11600429]

159. Ebihara L. Xenopus connexin38 forms hemi-gap-junctional channels in the nonjunctional plasma membrane of Xenopus oocytes. Biophys J. 1996; 71:742-748. [PubMed: 8842212]

160. Ebihara L, Liu X, Pal JD. Effect of external magnesium and calcium on human connexin 46 hemichannels. Biophys J. 2003; 84:277-286. [PubMed: 12524281]

161. Ebihara L, Steiner E. Properties of a nonjunctional current expressed from a rat connexin 46 cDNA in Xenopus oocytes. J Gen Physiol. 1993; 102:59-74. [PubMed: 7690837]

162. Eckert R. pH gating of lens fibre connexins. Pflugers Arch. 2002; 443:843-851. [PubMed: 11889584] 
163. Ek-Vitorin JF, Calero G, Morley GE, Coombs W, Taffet SM, Delmar M. PH regulation of connexin43: Molecular analysis of the gating particle. Biophys J. 1996; 71:1273-1284. [PubMed: 8874002]

164. Ek-Vitorin JF, King TJ, Heyman NS, Lampe PD, Burt JM. Selectivity of Connexin43 channels is regulated through protein kinase C-dependent phosphorylation. Circ Res. 2006; 98:1498-1505. [PubMed: 16709897]

165. Elden L, Bortoff A. Electrical coupling of longitudinal and circular intestinal muscle. Am J Physiol. 1984; 246:G618-G626. [PubMed: 6720957]

166. Elias LA, Kriegstein AR. Gap junctions: Multifaceted regulators of embryonic cortical development. Trends Neurosci. 2008; 31:243-250. [PubMed: 18403031]

167. Elias LA, Wang DD, Kriegstein AR. Gap junction adhesion is necessary for radial migration in the neocortex. Nature. 2007; 448:901-907. [PubMed: 17713529]

168. Eloff BC, Gilat E, Wan X, Rosenbaum DS. Pharmacological modulation of cardiac gap junctions to enhance cardiac conduction: Evidence supporting a novel target for antiarrhythmic therapy. Circulation. 2003; 108:3157-3163. [PubMed: 14656916]

169. Eltzschig HK, Eckle T, Mager A, Kuper N, Karcher C, Weissmuller T, Boengler K, Schulz R, Robson SC, Colgan SP. ATP release from activated neutrophils occurs via connexin 43 and modulates adenosinedependent endothelial cell function. Circ Res. 2006; 99:1100-1108. [PubMed: 17038639]

170. Eugenin EA, Branes MC, Berman JW, Saez JC. TNF-alpha plus IFNgamma induce connexin43 expression and formation of gap junctions between human monocytes/macrophages that enhance physiological responses. J Immunol. 2003; 170:1320-1328. [PubMed: 12538692]

171. Evans WH, Boitano S. Connexin mimetic peptides: Specific inhibitors of gap-junctional intercellular communication. Biochem Soc Trans. 2001; 29:606-612. [PubMed: 11498037]

172. Evans WH, De Vuyst E, Leybaert L. The gap junction cellular internet: Connexin hemichannels enter the signalling limelight. Biochem J. 2006; 397:1-14. [PubMed: 16761954]

173. Evans WH, Leybaert L. Mimetic peptides as blockers of connexin channel-facilitated intercellular communication. Cell Commun Adhes. 2007; 14:265-273. [PubMed: 18392994]

174. Faigle M, Seessle J, Zug S, El Kasmi KC, Eltzschig HK. ATP release from vascular endothelia occurs across Cx43 hemichannels and is attenuated during hypoxia. PLoS One. 2008; 3:e2801. [PubMed: 18665255]

175. Falk MM, Buehler LK, Kumar NM, Gilula NB. Cell-free synthesis and assembly of connexins into functional gap junction membrane channels. EMBO J. 1997; 16:2703-2716. [PubMed: 9184217]

176. Falk MM, Kumar NM, Gilula NB. Membrane insertion of gap junction connexins: Polytopic channel forming membrane proteins. J Cell Biol. 1994; 127:343-355. [PubMed: 7929580]

177. Fallon RF, Goodenough DA. Five-hour half-life of mouse liver gapjunction protein. J Cell Biol. 1981; 90:521-526. [PubMed: 7287816]

178. Farahani R, Pina-Benabou MH, Kyrozis A, Siddiq A, Barradas PC, Chiu FC, Cavalcante LA, Lai JC, Stanton PK, Rozental R. Alterations in metabolism and gap junction expression may determine the role of astrocytes as "good samaritans" or executioners. Glia. 2005; 50:351-361. [PubMed: 15846800]

179. Fill M, Copello JA. Ryanodine receptor calcium release channels. Physiol Rev. 2002; 82:893922. [PubMed: 12270947]

180. Fiorini C, Gilleron J, Carette D, Valette A, Tilloy A, Chevalier S, Segretain D, Pointis G. Accelerated internalization of junctional membrane proteins (connexin 43, N-cadherin and ZO-1) within endocytic vacuoles: An early event of DDT carcinogenicity. Biochim Biophys Acta. 2008; 1778:56-67. [PubMed: 17949680]

181. FitzHarris G, Baltz JM. Granulosa cells regulate intracellular $\mathrm{pH}$ of the murine growing oocyte via gap junctions: Development of independent homeostasis during oocyte growth. Development. 2006; 133:591-599. [PubMed: 16407396]

182. FitzHarris G, Siyanov V, Baltz JM. Granulosa cells regulate oocyte intracellular pH against acidosis in preantral follicles by multiple mechanisms. Development. 2007; 134:4283-4295. [PubMed: 17978006] 
183. Forge A, Becker D, Casalotti S, Edwards J, Evans WH, Lench N, Souter M. Gap junctions and connexin expression in the inner ear. Novartis Found Symp. 1999; 219:134-150. [PubMed: 10207902]

184. Francis D, Stergiopoulos K, Ek-Vitorin JF, Cao FL, Taffet SM, Delmar M. Connexin diversity and gap junction regulation by pHi. Dev Genet. 1999; 24:123-136. [PubMed: 10079516]

185. Frank M, Eiberger B, Janssen-Bienhold U, de Sevilla Muller LP, Tjarks A, Kim JS, Maschke S, Dobrowolski R, Sasse P, Weiler R, Fleischmann BK, Willecke K. Neuronal connexin-36 can functionally replace connexin-45 in mouse retina but not in the developing heart. J Cell Sci. 2010; 123:3605-3615. [PubMed: 20930146]

186. Frantseva MV, Kokarovtseva L, Naus CG, Carlen PL, MacFabe D, Perez Velazquez JL. Specific gap junctions enhance the neuronal vulnerability to brain traumatic injury. J Neurosci. 2002; 22:644-653. [PubMed: 11826094]

187. Frantseva MV, Kokarovtseva L, Perez Velazquez JL. Ischemia-induced brain damage depends on specific gap-junctional coupling. J Cereb Blood Flow Metab. 2002; 22:453-462. [PubMed: 11919516]

188. Frenz CM, Van De Water TR. Immunolocalization of connexin 26 in the developing mouse cochlea. Brain Res Brain Res Rev. 2000; 32:172-180. [PubMed: 10751667]

189. Friedman D, Strowbridge BW. Both electrical and chemical synapses mediate fast network oscillations in the olfactory bulb. J Neurophysiol. 2003; 89:2601-2610. [PubMed: 12740407]

190. Fu CT, Bechberger JF, Ozog MA, Perbal B, Naus CC. CCN3 (NOV) interacts with connexin43 in C6 glioma cells: Possible mechanism of connexin-mediated growth suppression. J Biol Chem. 2004; 279:36943-36950. [PubMed: 15213231]

191. Fujimoto K, Nagafuchi A, Tsukita S, Kuraoka A, Ohokuma A, Shibata Y. Dynamics of connexins, E-cadherin and alpha-catenin on cell membranes during gap junction formation. $\mathrm{J}$ Cell Sci. 1997; 110(Pt 3):311-322. [PubMed: 9057084]

192. Fukuda T. Structural organization of the gap junction network in the cerebral cortex. Neuroscientist. 2007; 13:199-207. [PubMed: 17519363]

193. Gaietta G, Deerinck TJ, Adams SR, Bouwer J, Tour O, Laird DW, Sosinsky GE, Tsien RY, Ellisman MH. Multicolor and electron microscopic imaging of connexin trafficking. Science. 2002; 296:503-507. [PubMed: 11964472]

194. Galarreta M, Hestrin S. Electrical synapses between GABA-releasing interneurons. Nat Rev Neurosci. 2001; 2:425-433. [PubMed: 11389476]

195. Garcia M, Knight MM. Cyclic loading opens hemichannels to release ATP as part of a chondrocyte mechanotransduction pathway. J Orthop Res. 2010; 28:510-515. [PubMed: 19890993]

196. Garcia-Dorado D, Rodriguez-Sinovas A, Ruiz-Meana M. Gap junctionmediated spread of cell injury and death during myocardial ischemiareperfusion. Cardiovasc Res. 2004; 61:386-401. [PubMed: 14962471]

197. Garfield RE, Daniel EE, Dukes M, Fitzgerald JD. Changes of gap junctions in myometrium of guinea pig at parturition and abortion. Can J Physiol Pharmacol. 1982; 60:335-341. [PubMed: 6951630]

198. Garfield RE, Hayashi RH. Appearance of gap junctions in the myometrium of women during labor. Am J Obstet Gynecol. 1981; 140:254-260. [PubMed: 7246625]

199. Garfield RE, Kannan MS, Daniel EE. Gap junction formation in myometrium: Control by estrogens, progesterone, and prostaglandins. Am J Physiol. 1980; 238:C81-C89. [PubMed: 7369350]

200. Garfield RE, Rabideau S, Challis JR, Daniel EE. Hormonal control of gap junction formation in sheep myometrium during parturition. Biol Reprod. 1979a; 21:999-1007. [PubMed: 526515]

201. Garfield RE, Rabideau S, Challis JR, Daniel EE. Ultrastructural basis for maintenance and termination of pregnancy. Am J Obstet Gynecol. 1979b; 133:308-315. [PubMed: 433992]

202. Garfield RE, Sims S, Daniel EE. Gap junctions: Their presence and necessity in myometrium during parturition. Science. 1977; 198:958-960. [PubMed: 929182]

203. Garfield RE, Sims SM, Kannan MS, Daniel EE. Possible role of gap junctions in activation of myometrium during parturition. Am J Physiol. 1978; 235:C168-C179. [PubMed: 727239] 
204. Garre JM, Retamal MA, Cassina P, Barbeito L, Bukauskas FF, Saez JC, Bennett MV, Abudara V. FGF-1 induces ATP release from spinal astrocytes in culture and opens pannexin and connexin hemichannels. Proc Natl Acad Sci U S A. 2010; 107:22659-22664. [PubMed: 21148774]

205. Gellhaus A, Dong X, Propson S, Maass K, Klein-Hitpass L, Kibschull M, Traub O, Willecke K, Perbal B, Lye SJ, Winterhager E. Connexin43 interacts with NOV: A possible mechanism for negative regulation of cell growth in choriocarcinoma cells. J Biol Chem. 2004; 279:3693136942. [PubMed: 15181016]

206. Gellhaus A, Wotzlaw C, Otto T, Fandrey J, Winterhager E. More insights into the CCN3/ Connexin43 interaction complex and its role for signaling. J Cell Biochem. 2010; 110:129-140. [PubMed: 20336664]

207. Genetos DC, Kephart CJ, Zhang Y, Yellowley CE, Donahue HJ. Oscillating fluid flow activation of gap junction hemichannels induces ATP release from MLO-Y4 osteocytes. J Cell Physiol. 2007; 212:207-214. [PubMed: 17301958]

208. George CH, Kendall JM, Evans WH. Intracellular trafficking pathways in the assembly of connexins into gap junctions. J Biol Chem. 1999; 274:8678-8685. [PubMed: 10085106]

209. Ghatnekar GS, O’Quinn MP, Jourdan LJ, Gurjarpadhye AA, Draughn RL, Gourdie RG. Connexin43 carboxyl-terminal peptides reduce scar progenitor and promote regenerative healing following skin wounding. Regen Med. 2009; 4:205-223. [PubMed: 19317641]

210. Giepmans BN. Gap junctions and connexin-interacting proteins. Cardiovasc Res. 2004; 62:233245. [PubMed: 15094344]

211. Giepmans BN. Role of connexin43-interacting proteins at gap junctions. Adv Cardiol. 2006; 42:41-56. [PubMed: 16646583]

212. Giepmans BNG, Hengeveld T, Postma FR, Moolenaar WH. Interaction of c-Src with gap junction protein Connexin-43. J Biol Chem. 2001; 276:8544-8549. [PubMed: 11124251]

213. Giessmann D, Theiss C, Breipohl W, Meller K. Decreased gap junctional communication in neurobiotin microinjected lens epithelial cells after taxol treatment. Anat Embryol (Berl). 2005; 209:391-400. [PubMed: 15864639]

214. Gilchrist RB, Lane M, Thompson JG. Oocyte-secreted factors: Regulators of cumulus cell function and oocyte quality. Hum Reprod Update. 2008; 14:159-177. [PubMed: 18175787]

215. Gilleron J, Carette D, Fiorini C, Dompierre J, Macia E, Denizot JP, Segretain D, Pointis G. The large GTPase dynamin2: A new player in connexin 43 gap junction endocytosis, recycling and degradation. Int J Biochem Cell Biol. 2011; 43:1208-1217. [PubMed: 21554976]

216. Gilleron J, Nebout M, Scarabelli L, Senegas-Balas F, Palmero S, Segretain D, Pointis G. A potential novel mechanism involving connexin 43 gap junction for control of sertoli cell proliferation by thyroid hormones. J Cell Physiol. 2006; 209:153-161. [PubMed: 16823880]

217. Ginzberg RD, Gilula NB. Modulation of cell junctions during differentiation of the chicken otocyst sensory epithelium. Dev Biol. 1979; 68:110-129. [PubMed: 437313]

218. Girao H, Catarino S, Pereira P. Eps 15 interacts with ubiquitinated $\mathrm{Cx} 43$ and mediates its internalization. Exp Cell Res. 2009; 315:3587-3597. [PubMed: 19835873]

219. Goldberg GS, Moreno AP, Lampe PD. Gap junctions between cells expressing connexin 43 or 32 show inverse permselectivity to adenosine and ATP. J Biol Chem. 2002; 277:36725-36730. [PubMed: 12119284]

220. Goldenberg RC, Fortes FS, Cristancho JM, Morales MM, Franci CR, Varanda WA, Campos de Carvalho AC. Modulation of gap junction mediated intercellular communication in TM3 Leydig cells. J Endocrinol. 2003; 177:327-335. [PubMed: 12740021]

221. Gollob MH, Jones DL, Krahn AD, Danis L, Gong XQ, Shao Q, Liu X, Veinot JP, Tang AS, Stewart AF, Tesson F, Klein GJ, Yee R, Skanes AC, Guiraudon GM, Ebihara L, Bai D. Somatic mutations in the connexin 40 gene (GJA5) in atrial fibrillation. N Engl J Med. 2006; 354:26772688. [PubMed: 16790700]

222. Gomes P, Srinivas SP, Van DW, Vereecke J, Himpens B. ATP release through connexin hemichannels in corneal endothelial cells. Invest Ophthalmol Vis Sci. 2005; 46:1208-1218. [PubMed: 15790881] 
223. Gomez-Hernandez JM, de MM, Larrosa B, Gonzalez D, Barrio LC. Molecular basis of calcium regulation in connexin-32 hemichannels. Proc Natl Acad Sci U S A. 2003; 100:16030-16035. [PubMed: 14663144]

224. Gong X, Baldo GJ, Kumar NM, Gilula NB, Mathias RT. Gap junctional coupling in lenses lacking alpha3 connexin. Proc Natl Acad Sci U S A. 1998; 95:15303-15308. [PubMed: 9860964]

225. Gong X, Li E, Klier G, Huang Q, Wu Y, Lei H, Kumar NM, Horwitz J, Gilula NB. Disruption of [alpha]3 Connexin Gene Leads to Proteolysis and Cataractogenesis in Mice. Cell. 1997; 91:833843. [PubMed: 9413992]

226. Gonzalez D, Gomez-Hernandez JM, Barrio LC. Species specificity of mammalian connexin-26 to form open voltage-gated hemichannels. FASEB J. 2006; 20:2329-2338. [PubMed: 17077310]

227. Gonzalez D, Gomez-Hernandez JM, Barrio LC. Molecular basis of voltage dependence of connexin channels:An integrative appraisal. Prog Biophys Mol Biol. 2007; 94:66-106. [PubMed: 17470374]

228. Goubaeva F, Mikami M, Giardina S, Ding B, Abe J, Yang J. Cardiac mitochondrial connexin 43 regulates apoptosis. Biochem Biophys Res Commun. 2007; 352:97-103. [PubMed: 17107662]

229. Gourdie RG, Green CR, Severs NJ, Thompson RP. Immunolabelling patterns of gap junction connexins in the developing and mature rat heart. Anat Embryol (Berl). 1992; 185:363-378. [PubMed: 1319120]

230. Granot I, Dekel N. The ovarian gap junction protein connexin43: regulation by gonadotropins. Trends Endocrinol Metab. 2002; 13:310-313. [PubMed: 12163234]

231. Gros D, Jarry-Guichard T, Ten Velde I, de Maziere A, van Kempen MJ, Davoust J, Briand JP, Moorman AF, Jongsma HJ. Restricted distribution of connexin40, a gap junctional protein, in mammalian heart. Circ Res. 1994; 74:839-851. [PubMed: 8156631]

232. Grummer R, Chwalisz K, Mulholland J, Traub O, Winterhager E. Regulation of connexin 26 and connexin43 expression in rat endometrium by ovarian steroid hormones. Biol Reprod. 1994; 51:1109-1116. [PubMed: 7888490]

233. Gu H, Ek-Vitorin JF, Taffet SM, Delmar M. UltraRapid communication: Coexpression of connexins 40 and 43 enhances the $\mathrm{pH}$ sensitivityof gap junctions: A model for synergistic interactions among connexins. Circ Res. 2000; 861100

234. Guerra JM, Everett TH, Lee KW, Wilson E, Olgin JE. Effects of the gap junction modifier rotigaptide (ZP123) on atrial conduction and vulnerability to atrial fibrillation. Circulation. 2006; 114:110-118. [PubMed: 16818812]

235. Guerrero PA, Schuessler RB, Davis LM, Beyer EC, Johnson CM, Yamada KA, Saffitz JE. Slow ventricular conduction in mice heterozygous for a connexin43 null mutation. J Clin Invest. 1997; 99:1991-1998. [PubMed: 9109444]

236. Guldenagel M, Ammermuller J, Feigenspan A, Teubner B, Degen J, Sohl G, Willecke K, Weiler R. Visual transmission deficits in mice with targeted disruption of the gap junction gene connexin36. J Neurosci. 2001; 21:6036-6044. [PubMed: 11487627]

237. Gumpert AM, Varco JS, Baker SM, Piehl M, Falk MM. Doublemembrane gap junction internalization requires the clathrin-mediated endocytic machinery. FEBS Lett. 2008; 582:28872892. [PubMed: 18656476]

238. Guo Y, Martinez-Williams C, Rannels DE. Gap junction-microtubule associations in rat alveolar epithelial cells. Am J Physiol Lung Cell Mol Physiol. 2003; 285:L1213-L1221. [PubMed: 14604851]

239. Gupta N, Wang H, McLeod TL, Naus CC, Kyurkchiev S, Advani S, Yu J, Perbal B, Weichselbaum RR. Inhibition of glioma cell growth and tumorigenic potential by CCN3 (NOV). Mol Pathol. 2001; 54:293-299. [PubMed: 11577170]

240. Gustafsson F, Holstein-Rathlou N-H. Conducted vasomotor responses in arterioles: Characteristics, mechanisms and physiological significance. Acta Physiol Scand. 1999; 167:1121. [PubMed: 10519972]

241. Guttman JA, Lin AE, Li Y, Bechberger J, Naus CC, Vogl AW, Finlay BB. Gap junction hemichannels contribute to the generation of diarrhoea during infectious enteric disease. Gut. 2010; 59:218-226. [PubMed: 19828465] 
242. Guyot A, Hanrahan JW. ATP release from human airway epithelial cells studied using a capillary cell culture system. J Physiol. 2002; 545:199-206. [PubMed: 12433960]

243. Haefliger JA, Demotz S, Braissant O, Suter E, Waeber B, Nicod P, Meda P. Connexins 40 and 43 are differentially regulated within the kidneys of rats with renovascular hypertension. Kidney Int. 2001; 60:190-201. [PubMed: 11422751]

244. Haefliger JA, Nicod P, Meda P. Contribution of connexins to the function of the vascular wall. Cardiovasc Res. 2004; 62:345-356. [PubMed: 15094354]

245. Hagen A, Dietze A, Dhein S. Human cardiac gap-junction coupling: Effects of antiarrhythmic peptide AAP10. Cardiovasc Res. 2009; 83:405-415. [PubMed: 19176598]

246. Hamelin R, Allagnat F, Haefliger JA, Meda P. Connexins, diabetes and the metabolic syndrome. Curr Protein Pept Sci. 2009; 10:18-29. [PubMed: 19275669]

247. Harks EG, de Roos AD, Peters PH, de Haan LH, Brouwer A, Ypey DL, van Zoelen EJ, Theuvenet AP. Fenamates: A novel class of reversible gap junction blockers. J Pharmacol Exp Ther. 2001; 298:1033-1041. [PubMed: 11504800]

248. Harris AL. Connexin channel permeability to cytoplasmic molecules. Prog Biophys Mol Biol. 2007; 94:120-143. [PubMed: 17470375]

249. Harris, AL.; Locke, D. Permeability of Connexin Channels. In: Harris, AL.; Locke, D., editors. Connexins. A Guide. Humana Press; New York: 2009. p. 165-206.

250. Harris AL, Spray DC, Bennett MV. Kinetic properties of a voltagedependent junctional conductance. J Gen Physiol. 1981; 77:95-117. [PubMed: 6259275]

251. Harris RJ, Symon L. Extracellular pH, potassium, and calcium activities in progressive ischaemia of rat cortex. J Cereb Blood Flow Metab. 1984; 4:178-186. [PubMed: 6725430]

252. Haugan K, Marcussen N, Kjolbye AL, Nielsen MS, Hennan JK, Petersen JS. Treatment with the gap junction modifier rotigaptide (ZP123) reduces infarct size in rats with chronic myocardial infarction. J Cardiovasc Pharmacol. 2006; 47:236-242. [PubMed: 16495761]

253. Haugan K, Miyamoto T, Takeishi Y, Kubota I, Nakayama J, Shimojo H, Hirose M. Rotigaptide (ZP123) improves atrial conduction slowing in chronic volume overload-induced dilated atria. Basic Clin Pharmacol Toxicol. 2006; 99:71-79. [PubMed: 16867174]

254. Haugan K, Olsen KB, Hartvig L, Petersen JS, Holstein-Rathlou NH, Hennan J, Nielsen MS. The antiarrhythmic peptide analogue ZP123 prevents atrial conduction slowing during metabolic stress. J Cardiovasc Electrophysiol. 2005; 16:537-545. [PubMed: 15877626]

255. Hawat G, Benderdour M, Rousseau G, Baroudi G. Connexin 43 mimetic peptide Gap26 confers protection to intact heart against myocardial ischemia injury. Pflugers Arch. 2010; 460:583-592. [PubMed: 20514543]

256. He DS, Jiang JX, Taffet SM, Burt JM. Formation of heteromeric gap junction channels by connexins 40 and 43 in vascular smooth muscle cells. Proc Natl Acad Sci U S A. 1999; 96:64956500. [PubMed: 10339616]

257. He LQ, Cai F, Liu Y, Liu MJ, Tan ZP, Pan Q, Fang FY, Liang dS, Wu LQ, Long ZG, Dai HP, Xia K, Xia JH, Zhang ZH. Cx31 is assembled and trafficked to cell surface by ER-Golgi pathway and degraded by proteasomal or lysosomal pathways. Cell Res. 2005; 15:455-464. [PubMed: 15987604]

258. Heinzel FR, Luo Y, Li X, Boengler K, Buechert A, Garcia-Dorado D, Di LF, Schulz R, Heusch G. Impairment of diazoxide-induced formation of reactive oxygen species and loss of cardioprotection in connexin 43 deficient mice. Circ Res. 2005; 97:583-586. [PubMed: 16100048]

259. Hendrix EM, Mao SJ, Everson W, Larsen WJ. Myometrial connexin 43 trafficking and gap junction assembly at term and in preterm labor. Mol Reprod Dev. 1992; 33:27-38. [PubMed: 1324698]

260. Hendrix EM, Myatt L, Sellers S, Russell PT, Larsen WJ. Steroid hormone regulation of rat myometrial gap junction formation: Effects on cx43 levels and trafficking. Biol Reprod. 1995; 52:547-560. [PubMed: 7756450]

261. Hennan JK, Swillo RE, Morgan GA, Keith JC Jr. Schaub RG, Smith RP, Feldman HS, Haugan K, Kantrowitz J, Wang PJ, Abu-Qare A, Butera J, Larsen BD, Crandall DL. Rotigaptide (ZP123) prevents spontaneous ventricular arrhythmias and reduces infarct size during myocardial 
ischemia/ reperfusion injury in open-chest dogs. J Pharmacol Exp Ther. 2006; 317:236-243. [PubMed: 16344331]

262. Hennan JK, Swillo RE, Morgan GA, Rossman EI, Kantrowitz J, Butera J, Petersen JS, Gardell SJ, Vlasuk GP. GAP-134 ([2S,4R]-1-[2-aminoacetyl]4-benzamidopyrrolidine-2-carboxylic acid) prevents spontaneous ventricular arrhythmias and reduces infarct size during myocardial ischemia/reperfusion injury in open-chest dogs. J Cardiovasc Pharmacol Ther. 2009; 14:207214. [PubMed: 19721133]

263. Hennemann H, Kozjek G, Dahl E, Nicholson B, Willecke K. Molecular cloning of mouse connexins26 and -32: Similar genomic organization but distinct promoter sequences of two gap junction genes. Eur J Cell Biol. 1992; 58:81-89. [PubMed: 1322820]

264. Hermoso M, Saez JC, Villalon M. Identification of gap junctions in the oviduct and regulation of connexins during development and by sexual hormones. Eur J Cell Biol. 1997; 74:1-9. [PubMed: 9309385]

265. Herve JC, Bourmeyster N, Sarrouilhe D, Duffy HS. Gap junctional complexes: From partners to functions. Prog Biophys Mol Biol. 2007; 94:29-65. [PubMed: 17507078]

266. Herve JC, Pluciennik F, Verrecchia F, Bastide B, Delage B, Joffre M, Deleze J. Influence of the molecular structure of steroids on their ability to interrupt gap junctional communication. $\mathrm{J}$ Membr Biol. 1996; 149:179-187. [PubMed: 8801350]

267. Hervé JC, Sarrouilhe D. Protein phosphatase modulation of the intercellular junctional communication: Importance in cardiac myocytes. Prog Biophys Mol Biol. 2006; 90:225-248. [PubMed: 16054199]

268. Hesketh GG, Shah MH, Halperin VL, Cooke CA, Akar FG, Yen TE, Kass DA, Machamer CE, Van Eyk JE, Tomaselli GF. Ultrastructure and regulation of lateralized connexin43 in the failing heart. Circ Res. 2010; 106:1153-1163. [PubMed: 20167932]

269. Hess DT, Matsumoto A, Kim SO, Marshall HE, Stamler JS. Protein S-nitrosylation: Purview and parameters. Nat Rev Mol Cell Biol. 2005; 6:150-166. [PubMed: 15688001]

270. Hibino H, Kurachi Y. Molecular and physiological bases of the K+ circulation in the mammalian inner ear. Physiology (Bethesda). 2006; 21:336-345. [PubMed: 16990454]

271. Hille B. Ionic channels in excitable membranes. Current problems and biophysical approaches. Biophys J. 1978; 22:283-294. [PubMed: 656545]

272. Hirai A, Yano T, Nishikawa K, Suzuki K, Asano R, Satoh H, Hagiwara K, Yamasaki H. Downregulation of connexin 32 gene expression through DNA methylation in a human renal cell carcinoma cell. Am J Nephrol. 2003; 23:172-177. [PubMed: 12690227]

273. Hirashiki A, Yamada Y, Murase Y, Suzuki Y, Kataoka H, Morimoto Y, Tajika T, Murohara T, Yokota M. Association of gene polymorphisms with coronary artery disease in low- or high-risk subjects defined by conventional risk factors. J Am Coll Cardiol. 2003; 42:1429-1437. [PubMed: 14563588]

274. Hirst-Jensen BJ, Sahoo P, Kieken F, Delmar M, Sorgen PL. Characterization of the pH-dependent interaction between the gap junction protein connexin 43 carboxyl terminus and cytoplasmic loop domains. J Biol Chem. 2007; 282:5801-5813. [PubMed: 17178730]

275. Hoh JH, Lal R, John SA, Revel JP, Arnsdorf MF. Atomic force microscopy and dissection of gap junctions. Science. 1991; 253:1405-1408. [PubMed: 1910206]

276. Hoh JH, Sosinsky GE, Revel JP, Hansma PK. Structure of the extracellular surface of the gap junction by atomic force microscopy. Biophys J. 1993; 65:149-163. [PubMed: 8396452]

277. Hohl M, Thiel G. Cell type-specific regulation of RE-1 silencing transcription factor (REST) target genes. Eur J Neurosci. 2005; 22:2216-2230. [PubMed: 16262660]

278. Hoogaars WM, Tessari A, Moorman AF, de Boer PA, Hagoort J, Soufan AT, Campione M, Christoffels VM. The transcriptional repressor Tbx3 delineates the developing central conduction system of the heart. Cardiovasc Res. 2004; 62:489-499. [PubMed: 15158141]

279. Hormuzdi SG, Pais I, Lebeau FE, Towers SK, Rozov A, Buhl EH, Whittington MA, Monyer H. Impaired electrical signaling disrupts gamma frequency oscillations in connexin 36-deficient mice. Neuron. 2001; 31:487-495. [PubMed: 11516404]

280. Hu X, Dahl G. Exchange of conductance and gating properties between gap junction hemichannels. FEBS Lett. 1999; 451:113-117. [PubMed: 10371149] 
281. Huang RP, Fan Y, Hossain MZ, Peng A, Zeng ZL, Boynton AL. Reversion of the neoplastic phenotype of human glioblastoma cells by connexin 43 (cx43). Cancer Res. 1998; 58:50895096. [PubMed: 9823317]

282. Huang RY, Laing JG, Kanter EM, Berthoud VM, Bao M, Rohrs HW, Townsend RR, Yamada KA. Identification of CaMKII phosphorylation sites in Connexin43 by high-resolution mass spectrometry. J Proteome Res. 2010; 10:1098-1109. [PubMed: 21158428]

283. Huang XD, Horackova M, Pressler ML. Changes in the expression and distribution of connexin 43 in isolated cultured adult guinea pig cardiomyocytes. Exp Cell Res. 1996; 228:254-261. [PubMed: 8912718]

284. Hunter AW, Barker RJ, Zhu C, Gourdie RG. Zonula occludens-1 alters connexin43 gap junction size and organization by influencing channel accretion. Mol Biol Cell. 2005; 16:5686-5698. [PubMed: 16195341]

285. Iacobas DA, Iacobas S, Spray DC. Connexin-dependent transcellular transcriptomic networks in mouse brain. Prog Biophys Mol Biol. 2007; 94:169-185. [PubMed: 17507080]

286. Inose H, Ochi H, Kimura A, Fujita K, Xu R, Sato S, Iwasaki M, Sunamura S, Takeuchi Y, Fukumoto S, Saito K, Nakamura T, Siomi H, Ito H, Arai Y, Shinomiya K, Takeda S. A microRNA regulatory mechanism of osteoblast differentiation. Proc Natl Acad Sci U S A. 2009; 106:20794-20799. [PubMed: 19933329]

287. Jain SK, Schuessler RB, Saffitz JE. Mechanisms of delayed electrical uncoupling induced by ischemic preconditioning. Circ Res. 2003; 92:1138-1144. [PubMed: 12730093]

288. Jansen JA, van Veen TA, de Bakker JM, van Rijen HV. Cardiac connexins and impulse propagation. J Mol Cell Cardiol. 2010; 48:76-82. [PubMed: 19729017]

289. Jedamzik B, Marten I, Ngezahayo A, Ernst A, Kolb HA. Regulation of lens rCx46-formed hemichannels by activation of protein kinase $\mathrm{C}$, external $\mathrm{Ca}(2+)$ and protons. J Membr Biol. 2000; 173:39-46. [PubMed: 10612690]

290. Jeyaraman M, Tanguy Sp, Fandrich RR, Lukas A, Kardami E. Ischemiainduced dephosphorylation of cardiomyocyte connexin-43 is reduced by okadaic acid and calyculin A but not fostriecin. Mol Cell Biochem. 2003; 242:129-134. [PubMed: 12619875]

291. Jiang JX, Goodenough DA. Phosphorylation of lens-fiber connexins in lens organ cultures. Eur J Biochem. 1998; 255:37-44. [PubMed: 9692898]

292. John S, Cesario D, Weiss JN. Gap junctional hemichannels in the heart. Acta Physiol Scand. 2003; 179:23-31. [PubMed: 12940935]

293. John SA, Kondo R, Wang SY, Goldhaber JI, Weiss JN. Connexin-43 hemichannels opened by metabolic inhibition. J Biol Chem. 1999; 274:236-240. [PubMed: 9867835]

294. John SA, Revel JP. Connexon integrity is maintained by non-covalent bonds: Intramolecular disulfide bonds link the extracellular domains in rat connexin-43. Biochem Biophys Res Commun. 1991; 178:1312-1318. [PubMed: 1651718]

295. Johnston MF, Simon SA, Ramon F. Interaction of anaesthetics with electrical synapses. Nature. 1980; 286:498-500. [PubMed: 6250068]

296. Jordan K, Chodock R, Hand AR, Laird DW. The origin of annular junctions: A mechanism of gap junction internalization. J Cell Sci. 2001; 114:763-773. [PubMed: 11171382]

297. Joshi-Mukherjee R, Coombs W, Burrer C, de Mora IA, Delmar M, Taffet SM. Evidence for the presence of a free C-terminal fragment of cx43 in cultured cells. Cell Commun Adhes. 2007; 14:75-84. [PubMed: 17668351]

298. Jude JA, Wylam ME, Walseth TF, Kannan MS. Calcium signaling in airway smooth muscle. Proc Am Thorac Soc. 2008; 5:15-22. [PubMed: 18094080]

299. Juneja SC, Barr KJ, Enders GC, Kidder GM. Defects in the germ line and gonads of mice lacking connexin43. Biol Reprod. 1999; 60:1263-1270. [PubMed: 10208994]

300. Kamasawa N, Sik A, Morita M, Yasumura T, Davidson KG, Nagy JI, Rash JE. Connexin-47 and connexin-32 in gap junctions of oligodendrocyte somata, myelin sheaths, paranodal loops and Schmidt-Lanterman incisures: Implications for ionic homeostasis and potassium siphoning. Neuroscience. 2005; 136:65-86. [PubMed: 16203097]

301. Kang J, Kang N, Lovatt D, Torres A, Zhao Z, Lin J, Nedergaard M. Connexin 43 hemichannels are permeable to ATP. J Neurosci. 2008; 28:4702-4711. [PubMed: 18448647] 
302. Kanno S, Kovacs A, Yamada KA, Saffitz JE. Connexin43 as a determinant of myocardial infarct size following coronary occlusion in mice. J Am Coll Cardiol. 2003; 41:681-686. [PubMed: 12598083]

303. Kardami E, Dang X, Iacobas DA, Nickel BE, Jeyaraman M, Srisakuldee W, Makazan J, Tanguy S, Spray DC. The role of connexins in controlling cell growth and gene expression. Prog Biophys Mol Biol. 2007; 94:245-264. [PubMed: 17462721]

304. Kathiriya IS, King IN, Murakami M, Nakagawa M, Astle JM, Gardner KA, Gerard RD, Olson EN, Srivastava D, Nakagawa O. Hairy-related transcription factors inhibit GATA-dependent cardiac gene expression through a signal-responsive mechanism. J Biol Chem. 2004; 279:5493754943. [PubMed: 15485867]

305. Katsuragi T, Migita K. The mechanism of ATP release as an autocrine/paracrine molecule. Nippon Yakurigaku Zasshi. 2004; 123:382-388. [PubMed: 15170077]

306. Kedde M, Strasser MJ, Boldajipour B, Oude Vrielink JA, Slanchev K, le SC, Nagel R, Voorhoeve PM, van DJ, Orom UA, Lund AH, Perrakis A, Raz E, Agami R. RNA-binding protein Dnd1 inhibits microRNA access to target mRNA. Cell. 2007; 131:1273-1286. [PubMed: 18155131]

307. Kelsell DP, Di WL, Houseman MJ. Connexin mutations in skin disease and hearing loss. Am J Hum Genet. 2001; 68:559-568. [PubMed: 11179004]

308. Kelsell DP, Dunlop J, Stevens HP, Lench NJ, Liang JN, Parry G, Mueller RF, Leigh IM. Connexin 26 mutations in hereditary non-syndromic sensorineural deafness. Nature. 1997; 387:80-83. [PubMed: 9139825]

309. Kidder GM, Vanderhyden BC. Bidirectional communication between oocytes and follicle cells: Ensuring oocyte developmental competence. Can J Physiol Pharmacol. 2010; 88:399-413. [PubMed: 20555408]

310. Kim DY, Kam Y, Koo SK, Joe CO. Gating connexin 43 channels reconstituted in lipid vesicles by mitogen-activated protein kinase phosphorylation. J Biol Chem. 1999; 274:5581-5587. [PubMed: 10026174]

311. Kim HK, Lee YS, Sivaprasad U, Malhotra A, Dutta A. Muscle-specific microRNA miR-206 promotes muscle differentiation. J Cell Biol. 2006; 174:677-687. [PubMed: 16923828]

312. Kimura K, Nishida T. Role of the ubiquitin-proteasome pathway in downregulation of the gapjunction protein Connexin43 by TNF- $\{$ alpha $\}$ in human corneal fibroblasts. Invest Ophthalmol Vis Sci. 2010; 51:1943-1947. [PubMed: 19907029]

313. King TJ, Fukushima LH, Donlon TA, Hieber AD, Shimabukuro KA, Bertram JS. Correlation between growth control, neoplastic potential and endogenous connexin43 expression in HeLa cell lines: Implications for tumor progression. Carcinogenesis. 2000; 21:311-315. [PubMed: 10657974]

314. King TJ, Gurley KE, Prunty J, Shin JL, Kemp CJ, Lampe PD. Deficiency in the gap junction protein connexin32 alters p27Kip1 tumor suppression and MAPK activation in a tissue-specific manner. Oncogene. 2005; 24:1718-1726. [PubMed: 15608667]

315. Kistler J, Kirkland B, Bullivant S. Identification of a 70,000-D protein in lens membrane junctional domains. J Cell Biol. 1985; 101:28-35. [PubMed: 3891760]

316. Kistler WM, de Jeu MT, Elgersma Y, van der Giessen RS, Hensbroek R, Luo C, Koekkoek SK, Hoogenraad CC, Hamers FP, Gueldenagel M, Sohl G, Willecke K, De Zeeuw CI. Analysis of Cx36 knockout does not support tenet that olivary gap junctions are required for complex spike synchronization and normal motor performance. Ann N Y Acad Sci. 2002; 978:391-404. [PubMed: 12582068]

317. Kjenseth A, Fykerud T, Rivedal E, Leithe E. Regulation of gap junction intercellular communication by the ubiquitin system. Cell Signal. 2010; 22:1267-1273. [PubMed: 20206687]

318. Kjolbye AL, Dikshteyn M, Eloff BC, Deschenes I, Rosenbaum DS. Maintenance of intercellular coupling by the antiarrhythmic peptide rotigaptide suppresses arrhythmogenic discordant alternans. Am J Physiol Heart Circ Physiol. 2008; 294:H41-H49. [PubMed: 17982010]

319. Kjolbye AL, Haugan K, Hennan JK, Petersen JS. Pharmacological modulation of gap junction function with the novel compound rotigaptide: A promising new principle for prevention of arrhythmias. Basic Clin Pharmacol Toxicol. 2007; 101:215-230. [PubMed: 17845503] 
320. Kjolbye AL, Holstein-Rathlou NH, Petersen JS. Anti-arrhythmic peptide N-3-(4hydroxyphenyl)propionyl Pro-Hyp-Gly-Ala-Gly-OH reduces dispersion of action potential duration during ischemia/ reperfusion in rabbit hearts. J Cardiovasc Pharmacol. 2002; 40:770779. [PubMed: 12409986]

321. Kjolbye AL, Knudsen CB, Jepsen T, Larsen BD, Petersen JS. Pharmacological characterization of the new stable antiarrhythmic peptide analog Ac-D-Tyr-D-Pro-D-Hyp-Gly-D-Ala-Gly-NH2 (ZP123): in vivo and in vitro studies. J Pharmacol Exp Ther. 2003; 306:1191-1199. [PubMed: 12829724]

322. Kleber AG, Rudy Y. Basic mechanisms of cardiac impulse propagation and associated arrhythmias. Physiol Rev. 2004; 84:431-488. [PubMed: 15044680]

323. Kleopa KA, Orthmann-Murphy J, Sargiannidou I. Gap junction disorders of myelinating cells. Rev Neurosci. 2010; 21:397-419. [PubMed: 21280457]

324. Koffler LD, Fernstrom MJ, Akiyama TE, Gonzalez FJ, Ruch RJ. Positive regulation of connexin32 transcription by hepatocyte nuclear factor-1alpha. Arch Biochem Biophys. 2002; 407:160-167. [PubMed: 12413486]

325. Koibuchi N, Chin MT. CHF1/Hey2 plays a pivotal role in left ventricular maturation through suppression of ectopic atrial gene expression. Circ Res. 2007; 100:850-855. [PubMed: 17332425]

326. Kojima T, Srinivas M, Fort A, Hopperstad M, Urban M, Hertzberg EL, Mochizuki Y, Spray DC. TPA induced expression and function of human connexin 26 by post-translational mechanisms in stably transfected neuroblastoma cells. Cell Struct Funct. 1999; 24:435-441. [PubMed: 10698257]

327. Kondo RP, Wang SY, John SA, Weiss JN, Goldhaber JI. Metabolic inhibition activates a nonselective current through connexin hemichannels in isolated ventricular myocytes. J Mol Cell Cardiol. 2000; 32:1859-1872. [PubMed: 11013130]

328. Koval M, Harley JE, Hick E, Steinberg TH. Connexin46 is retained as monomers in a trans-Golgi compartment of osteoblastic cells. J Cell Biol. 1997; 137:847-857. [PubMed: 9151687]

329. Kozoriz MG, Bechberger JF, Bechberger GR, Suen MW, Moreno AP, Maass K, Willecke K, Naus CC. The connexin43 C-terminal region mediates neuroprotection during stroke. J Neuropathol Exp Neurol. 2010; 69:196-206. [PubMed: 20084014]

330. Krenacs T, Rosendaal M. Connexin43 gap junctions in normal, regenerating, and cultured mouse bone marrow and in human leukemias: Their possible involvement in blood formation. Am $\mathbf{J}$ Pathol. 1998; 152:993-1004. [PubMed: 9546360]

331. Krenacs T, van DM, Lindhout E, Rosendaal M. Direct cell/cell communication in the lymphoid germinal center: Connexin43 gap junctions functionally couple follicular dendritic cells to each other and to B lymphocytes. Eur J Immunol. 1997; 27:1489-1497. [PubMed: 9209502]

332. Kreusch A, Pfaffinger PJ, Stevens CF, Choe S. Crystal structure of the tetramerization domain of the Shaker potassium channel. Nature. 1998; 392:945-948. [PubMed: 9582078]

333. Kreuzberg MM, Liebermann M, Segschneider S, Dobrowolski R, Dobrzynski H, Kaba R, Rowlinson G, Dupont E, Severs NJ, Willecke K. Human connexin31.9, unlike its orthologous protein connexin 30.2 in the mouse, is not detectable in the human cardiac conduction system. $\mathrm{J}$ Mol Cell Cardiol. 2009; 46:553-559. [PubMed: 19168070]

334. Kreuzberg MM, Schrickel JW, Ghanem A, Kim JS, Degen J, Janssen-Bienhold U, Lewalter T, Tiemann K, Willecke K. Connexin30.2 containing gap junction channels decelerate impulse propagation through the atrioventricular node. Proc Natl Acad Sci U S A. 2006; 103:5959-5964. [PubMed: 16571663]

335. Kreuzberg MM, Willecke K, Bukauskas FF. Connexin-mediated cardiac impulse propagation: Connexin 30.2 slows atrioventricular conduction in mouse heart. Trends Cardiovasc Med. 2006; 16:266-272. [PubMed: 17055382]

336. Kruger O, Plum A, Kim JS, Winterhager E, Maxeiner S, Hallas G, Kirchhoff S, Traub O, Lamers WH, Willecke K. Defective vascular development in connexin 45-deficient mice. Development. 2000; 127:4179-4193. [PubMed: 10976050]

337. Kumar NM, Gilula NB. Molecular biology and genetics of gap junction channels. Semin Cell Biol. 1992; 3:3-16. [PubMed: 1320430] 
338. Kumar NM, Gilula NB. The gap junction communication channel. Cell. 1996; 84:381-388. [PubMed: 8608591]

339. Kuraoka A, Yamanaka I, Miyahara A, Shibata Y, Uemura T. Immunocytochemical studies of major gap junction proteins in rat salivary glands. Eur Arch Otorhinolaryngol. 1994; 251(Suppl 1):S95-S99. [PubMed: 11894787]

340. Kurtz L, Schweda F, de WC, Kriz W, Witzgall R, Warth R, Sauter A, Kurtz A, Wagner C. Lack of connexin 40 causes displacement of renin-producing cells from afferent arterioles to the extraglomerular mesangium. J Am Soc Nephrol. 2007; 18:1103-1111. [PubMed: 17329574]

341. Kwak BR, Hermans MM, De Jonge HR, Lohmann SM, Jongsma HJ, Chanson M. Differential regulation of distinct types of gap junction channels by similar phosphorylating conditions. Mol Biol Cell. 1995; 6:1707-1719. [PubMed: 8590800]

342. Kwak BR, Jongsma HJ. Regulation of cardiac gap junction channel permeability and conductance by several phosphorylating conditions. Mol Cell Biochem. 1996; 157:93-99. [PubMed: 8739233]

343. Kwak BR, Mulhaupt F, Veillard N, Gros DB, Mach F. Altered pattern of vascular connexin expression in atherosclerotic plaques. Arterioscler Thromb Vasc Biol. 2002; 22:225-230. [PubMed: 11834520]

344. Kwak BR, Saez JC, Wilders R, Chanson M, Fishman GI, Hertzberg EL, Spray DC, Jongsma HJ. Effects of cGMP-dependent phosphorylation on rat and human connexin43 gap junction channels. Eur J Physiol. 1995; 430:770-778.

345. Kwak BR, Veillard N, Pelli G, Mulhaupt F, James RW, Chanson M, Mach F. Reduced connexin43 expression inhibits atherosclerotic lesion formation in low-density lipoprotein receptor-deficient mice. Circulation. 2003; 107:1033-1039. [PubMed: 12600918]

346. Kwak BR, van Veen TAB, Analbers LJS, Jongsma HJ. TPA increases conductance but decreases permeability in neonatal rat cardiomyocyte gap junction channels. Exp Cell Res. 1995; 220:456463. [PubMed: 7556455]

347. Kwong KF, Schuessler RB, Green KG, Laing JG, Beyer EC, Boineau JP, Saffitz JE. Differential expression of gap junction proteins in the canine sinus node. Circ Res. 1998; 82:604-612. [PubMed: 9529165]

348. Laing JG, Beyer EC. The gap junction protein connexin43 is degraded via the ubiquitin proteasome pathway. J Biol Chem. 1995; 270:26399-26403. [PubMed: 7592854]

349. Laing JG, Tadros PN, Green K, Saffitz JE, Beyer EC. Proteolysis of connexin43-containing gap junctions in normal and heat-stressed cardiac myocytes. Cardiovasc Res. 1998; 38:711-718. [PubMed: 9747439]

350. Laing JG, Tadros PN, Westphale EM, Beyer EC. Degradation of connexin43 gap junctions involves both the proteasome and the lysosome. Exp Cell Res. 1997; 236:482-492. [PubMed: 9367633]

351. Laird DW. Life cycle of connexins in health and disease. Biochem J. 2006; 394:527-543. [PubMed: 16492141]

352. Laird DW. The gap junction proteome and its relationship to disease. Trends Cell Biol. 2010; 20:92-101. [PubMed: 19944606]

353. Laird DW, Puranam KL, Revel JP. Turnover and phosphorylation dynamics of connexin43 gap junction protein in cultured cardiac myocytes. Biochem J. 1991; 273(Pt 1):67-72. [PubMed: 1846532]

354. Lampe PD, TenBroek EM, Burt JM, Kurata WE, Johnson RG. Phosphorylation of Connexin43 on Serine368 by protein kinase $\mathrm{C}$ regulates gap junctional communication. J Cell Biol. 2000; 149:1503-1512. [PubMed: 10871288]

355. Lampe PD, Cooper CD, King TJ, Burt JM. Analysis of Connexin43 phosphorylated at S325, S328 and S330 in normoxic and ischemic heart. J Cell Sci. 2006; 119:3435-3442. [PubMed: 16882687]

356. Lampe PD, Lau AF. Regulation of gap junctions by phosphorylation of connexins. Arch Biochem Biophys. 2000; 384:205-215. [PubMed: 11368307]

357. Lampe PD, Lau AF. The effects of connexin phosphorylation on gap junctional communication. Int J Biochem Cell Biol. 2004; 36:1171-1186. [PubMed: 15109565] 
358. Langlois S, Cowan KN, Shao Q, Cowan BJ, Laird DW. Caveolin-1 and -2 interact with connexin43 and regulate gap junctional intercellular communication in keratinocytes. Mol Biol Cell. 2008; 19:912-928. [PubMed: 18162583]

359. Larsen WJ, Tung HN, Murray SA, Swenson CA. Evidence for the participation of actin microfilaments and bristle coats in the internalization of gap junction membrane. J Cell Biol. 1979; 83:576-587. [PubMed: 574870]

360. Lauf U, Giepmans BN, Lopez P, Braconnot S, Chen SC, Falk MM. Dynamic trafficking and delivery of connexons to the plasma membrane and accretion to gap junctions in living cells. Proc Natl Acad Sci U S A. 2002; 99:10446-10451. [PubMed: 12149451]

361. Laurent G, Leong-Poi H, Mangat I, Moe GW, Hu X, So PP, Tarulli E, Ramadeen A, Rossman EI, Hennan JK, Dorian P. Effects of chronic gap junction conduction-enhancing antiarrhythmic peptide GAP-134 administration on experimental atrial fibrillation in dogs. Circ Arrhythm Electrophysiol. 2009; 2:171-178. [PubMed: 19808462]

362. Law LY, Zhang WV, Stott NS, Becker DL, Green CR. In vitro optimization of antisense oligodeoxynucleotide design: An example using the connexin gene family. J Biomol Tech. 2006; 17:270-282. [PubMed: 17028167]

363. Lazrak A, Peracchia C. Gap junction gating sensitivity to physiological internal calcium regardless of pH in Novikoff hepatoma cells. Biophys J. 1993; 65:2002-2012. [PubMed: 8298030]

364. Lee SM, Clemens MG. Subacinar distribution of hepatocyte membrane potential response to stimulation of gluconeogenesis. Am J Physiol. 1992; 263:G319-G326. [PubMed: 1415543]

365. Lefebvre PP, Van DeWater TR. Connexins, hearing and deafness: Clinical aspects of mutations in the connexin 26 gene. Brain Res Brain Res Rev. 2000; 32:159-162. [PubMed: 10928803]

366. Leithe E, Kjenseth A, Sirnes S, Stenmark H, Brech A, Rivedal E. Ubiquitylation of the gap junction protein connexin-43 signals its trafficking from early endosomes to lysosomes in a process mediated by Hrs and Tsg101. J Cell Sci. 2009; 122:3883-3893. [PubMed: 19808888]

367. Leithe E, Rivedal E. Epidermal growth factor regulates ubiquitination, internalization and proteasome-dependent degradation of connexin43. J Cell Sci. 2004a; 117:1211-1220. [PubMed: 14970263]

368. Leithe E, Rivedal E. Ubiquitination and down-regulation of gap junction protein connexin-43 in response to 12-O-tetradecanoylphorbol 13-acetate treatment. J Biol Chem. 2004b; 279:5008950096. [PubMed: 15371442]

369. Lerner DL, Yamada KA, Schuessler RB, Saffitz JE. Accelerated onset and increased incidence of ventricular arrhythmias induced by ischemia in Cx43-deficient mice. Circulation. 2000; 101:547552. [PubMed: 10662753]

370. Lewandowski R, Procida K, Vaidyanathan R, Coombs W, Jalife J, Nielsen MS, Taffet SM, Delmar M. RXP-E: A connexin43-binding peptide that prevents action potential propagation block. Circ Res. 2008; 103:519-526. [PubMed: 18669919]

371. Leybaert L, Braet K, Vandamme W, Cabooter L, Martin PE, Evans WH. Connexin channels, connexin mimetic peptides and ATP release. Cell Commun Adhes. 2003; 10:251-257. [PubMed: 14681025]

372. Leykauf K, Salek M, Bomke J, Frech M, Lehmann WD, Durst M, Alonso A. Ubiquitin protein ligase Nedd4 binds to connexin43 by a phosphorylation-modulated process. J Cell Sci. 2006; 119:3634-3642. [PubMed: 16931598]

373. Li A, Leung CT, Peterson-Yantorno K, Mitchell CH, Civan MM. Pathways for ATP release by bovine ciliary epithelial cells, the initial step in purinergic regulation of aqueous humor inflow. Am J Physiol Cell Physiol. 2010; 299:C1308-C1317. [PubMed: 20926783]

374. Li F, Sugishita K, Su Z, Ueda I, Barry WH. Activation of connexin-43 hemichannels can elevate $[\mathrm{Ca}(2+)] \mathrm{i}$ and $[\mathrm{Na}(+)] \mathrm{i}$ in rabbit ventricular myocytes during metabolic inhibition. J Mol Cell Cardiol. 2001; 33:2145-2155. [PubMed: 11735261]

375. Li H, Brodsky S, Kumari S, Valiunas V, Brink P, Kaide J, Nasjletti A, Goligorsky MS. Paradoxical overexpression and translocation of connexin43 in homocysteine-treated endothelial cells. Am J Physiol Heart Circ Physiol. 2002; 282:H2124-H2133. [PubMed: 12003820] 
376. Li H, Liu TF, Lazrak A, Peracchia C, Goldberg GS, Lampe PD, Johnson RG. Properties and regulation of gap junctional hemichannels in the plasma membranes of cultured cells. J Cell Biol. 1996; 134:1019-1030. [PubMed: 8769424]

377. Li X, Heinzel FR, Boengler K, Schulz R, Heusch G. Role of connexin 43 in ischemic preconditioning does not involve intercellular communication through gap junctions. J Mol Cell Cardiol. 2004; 36:161-163. [PubMed: 14734058]

378. Li X, Su V, Kurata WE, Jin C, Lau AF. A novel connexin43-interacting protein, CIP75, which belongs to the UbL-UBAprotein family, regulates the turnover of connexin43. J Biol Chem. 2008; 283:5748-5759. [PubMed: 18079109]

379. Lichtenstein A, Minogue PJ, Beyer EC, Berthoud VM. Autophagy: A pathway that contributes to connexin degradation. J Cell Sci. 2011; 124:910-920. [PubMed: 21378309]

380. Lin D, Lobell S, Jewell A, Takemoto DJ. Differential phosphorylation of connexin 46 and connexin50 by H2O2 activation of protein kinase Cgamma. Mol Vis. 2004; 10:688-695. [PubMed: 15467523]

381. Lin JH, Lou N, Kang N, Takano T, Hu F, Han X, Xu Q, Lovatt D, Torres A, Willecke K, Yang J, Kang J, Nedergaard M. A central role of connexin 43 in hypoxic preconditioning. J Neurosci. 2008; 28:681-695. [PubMed: 18199768]

382. Lin JS, Fitzgerald S, Dong YM, Knight C, Donaldson P, Kistler J. Processing of the gap junction protein connexin50 in the ocular lens is accomplished by calpain. Eur J Cell Biol. 1997; 73:141149. [PubMed: 9208227]

383. Lin R, Warn-Cramer BJ, Kurata WE, Lau AF. v-Src phosphorylation of connexin 43 on Tyr247 and Tyr265 disrupts gap junctional communication. J Cell Biol. 2001; 154:815-828. [PubMed: 11514593]

384. Lin X, Zemlin C, Hennan JK, Petersen JS, Veenstra RD. Enhancement of ventricular gap-junction coupling by rotigaptide. Cardiovasc Res. 2008; 79:416-426. [PubMed: 18430749]

385. Linhares VL, Almeida NA, Menezes DC, Elliott DA, Lai D, Beyer EC, Campos de Carvalho AC, Costa MW. Transcriptional regulation of the murine Connexin 40 promoter by cardiac factors Nkx2-5, GATA4 and Tbx5. Cardiovasc Res. 2004; 64:402-411. [PubMed: 15537493]

386. Listi F, Candore G, Balistreri CR, Caruso M, Incalcaterra E, Hoffmann E, Lio D, Caruso C. Connexin37 1019 gene polymorphism in myocardial infarction patients and centenarians. Atherosclerosis. 2007; 191:460-461. [PubMed: 16970956]

387. Liu F, Arce FT, Ramachandran S, Lal R. Nanomechanics of hemichannel conformations: Connexin flexibility underlying channel opening and closing. J Biol Chem. 2006; 281:2320723217. [PubMed: 16769719]

388. Liu HT, Tashmukhamedov BA, Inoue H, Okada Y, Sabirov RZ. Roles of two types of anion channels in glutamate release from mouse astrocytes under ischemic or osmotic stress. Glia. 2006; 54:343-357. [PubMed: 16883573]

389. Liu J, Xu J, Gu S, Nicholson BJ, Jiang JX. Aquaporin 0 enhances gap junction coupling via its cell adhesion function and interaction with connexin 50. J Cell Sci. 2011; 124:198-206. [PubMed: 21172802]

390. Liu LW, Farraway L, Berezin I, Huizinga JD. Interstitial cells of Cajal: Mediators of communication between circular and longitudinal muscle layers of canine colon. Cell Tissue Res. 1998; 294:69-79. [PubMed: 9724457]

391. Liu S, Taffet S, Stoner L, Delmar M, Vallano ML, Jalife J. A structural basis for the unequal sensitivity of the major cardiac and liver gap junctions to intracellular acidification: The carboxyl tail length. Biophys J. 1993; 64:1422-1433. [PubMed: 8391867]

392. Lobo IA, Mascia MP, Trudell JR, Harris RA. Channel gating of the glycine receptor changes accessibility to residues implicated in receptor potentiation by alcohols and anesthetics. J Biol Chem. 2004; 279:33919-33927. [PubMed: 15169788]

393. Locke D, Bian S, Li H, Harris AL. Post-translational modifications of connexin26 revealed by mass spectrometry. Biochem J. 2009; 424:385-398. [PubMed: 19775242]

394. Locke D, Koreen IV, Harris AL. Isoelectric points and post-translational modifications of connexin26 and connexin32. FASEB J. 2006; 20:1221-1223. [PubMed: 16645047] 
395. Locovei S, Bao L, Dahl G. Pannexin 1 in erythrocytes: Function without a gap. Proc Natl Acad Sci U S A. 2006; 103:7655-7659. [PubMed: 16682648]

396. Loewenstein WR, Kanno Y. Intercellular communication and the control of tissue growth: Lack of communication between cancer cells. Nature. 1966; 209:1248-1249. [PubMed: 5956321]

397. Loncarek J, Yamasaki H, Levillain P, Milinkevitch S, Mesnil M. The expression of the tumor suppressor gene connexin 26 is not mediated by methylation in human esophageal cancer cells. Mol Carcinog. 2003; 36:74-81. [PubMed: 12557263]

398. Long MA, Deans MR, Paul DL, Connors BW. Rhythmicity without synchrony in the electrically uncoupled inferior olive. J Neurosci. 2002; 22:10898-10905. [PubMed: 12486184]

399. Lu G, Haider HK, Porollo A, Ashraf M. Mitochondria-specific transgenic overexpression of connexin-43 simulates preconditioning-induced cytoprotection of stem cells. Cardiovasc Res. 2010; 88:277-286. [PubMed: 20833648]

400. Lu Y, Zhang Y, Shan H, Pan Z, Li X, Li B, Xu C, Zhang B, Zhang F, Dong D, Song W, Qiao G, Yang B. MicroRNA-1 downregulation by propranolol in a rat model of myocardial infarction: A new mechanism for ischaemic cardioprotection. Cardiovasc Res. 2009; 84:434-441. [PubMed: 19581315]

401. Luk JM, Mok BW, Shum CK, Yeung WS, Tam PC, Tse JY, Chow JF, Woo J, Kam K, Lee KF. Identification of novel genes expressed during spermatogenesis in stage-synchronized rat testes by differential display. Biochem Biophys Res Commun. 2003; 307:782-790. [PubMed: 12878178]

402. Lurtz MM, Louis CF. Intracellular calcium regulation of connexin43. Am J Physiol Cell Physiol. 2007; 293:C1806-C1813. [PubMed: 17898133]

403. Maass K, Chase SE, Lin X, Delmar M. Cx43 CT domain influences infarct size and susceptibility to ventricular tachyarrhythmias in acute myocardial infarction. Cardiovasc Res. 2009; 84:361367. [PubMed: 19620131]

404. Macia E, Dolmatova E, Cabo C, Sosinsky AZ, Dun W, Coromilas J, Ciaccio EJ, Boyden PA, Wit AL, Duffy HS. Characterization of gap junction remodeling in epicardial border zone of healing canine infarcts and electrophysiological effects of partial reversal by rotigaptide. Circ Arrhythm Electrophysiol. 2011; 4:344-351. [PubMed: 21493965]

405. Mackay D, Ionides A, Kibar Z, Rouleau G, Berry V, Moore A, Shiels A, Bhattacharya S. Connexin46 mutations in autosomal dominant congenital cataract. Am J Hum Genet. 1999; 64:1357-1364. [PubMed: 10205266]

406. Mackenzie LW, Garfield RE. Hormonal control of gap junctions in the myometrium. Am J Physiol. 1985; 248:C296-C308. [PubMed: 3976879]

407. Mackenzie LW, Garfield RE. Effects of 17 beta-estradiol on myometrial gap junctions and pregnancy in the rat. Can J Physiol Pharmacol. 1986; 64:462-466. [PubMed: 3730929]

408. Mackenzie LW, Puri CP, Garfield RE. Effect of estradiol-17 beta and prostaglandins on rat myometrial gap junctions. Prostaglandins. 1983; 26:925-941. [PubMed: 6597494]

409. Maeda S, Nakagawa S, Suga M, Yamashita E, Oshima A, Fujiyoshi Y, Tsukihara T. Structure of the connexin 26 gap junction channel at 3.5 A resolution. Nature. 2009; 458:597-602. [PubMed: 19340074]

410. Maier LS. CaMKIId overexpression in hypertrophy and heart failure: Cellular consequences for excitation-contraction coupling. Braz J Med Biol Res. 2005; 38:1293-1302. [PubMed: 16138211]

411. Majoul IV, Onichtchouk D, Butkevich E, Wenzel D, Chailakhyan LM, Duden R. Limiting transport steps and novel interactions of Connexin-43 along the secretory pathway. Histochem Cell Biol. 2009; 132:263-280. [PubMed: 19626334]

412. Majumder P, Crispino G, Rodriguez L, Ciubotaru CD, Anselmi F, Piazza V, Bortolozzi M, Mammano F. ATP-mediated cell-cell signaling in the organ of Corti: The role of connexin channels. Purinergic Signal. 2010; 6:167-187. [PubMed: 20806010]

413. Makowski L, Caspar DL, Phillips WC, Goodenough DA. Gap junction structures. V. Structural chemistry inferred from X-ray diffraction measurements on sucrose accessibility and trypsin susceptibility. J Mol Biol. 1984; 174:449-481. [PubMed: 6716484] 
414. Malchow RP, Qian H, Haugh-Scheidt LM, Ripps H. The effects of quinine and quinidine on isolated retinal horizontal cells. Biol Bull. 1994; 187:262-263. [PubMed: 7811815]

415. Malchow RP, Qian H, Ripps H. A novel action of quinine and quinidine on the membrane conductance of neurons from the vertebrate retina. J Gen Physiol. 1994; 104:1039-1055. [PubMed: 7535344]

416. Martin D, Tawadros T, Meylan L, Abderrahmani A, Condorelli DF, Waeber G, Haefliger JA. Critical role of the transcriptional repressor neuron-restrictive silencer factor in the specific control of connexin36 in insulin-producing cell lines. J Biol Chem. 2003; 278:53082-53089. [PubMed: 14565956]

417. Martin PE, George CH, Castro C, Kendall JM, Capel J, Campbell AK, Revilla A, Barrio LC, Evans WH. Assembly of chimeric connexin-aequorin proteins into functional gap junction channels. Reporting intracellular and plasma membrane calcium environments. J Biol Chem. 1998; 273:1719-1726. [PubMed: 9430718]

418. Martinez AD, Acuna R, Figueroa V, Maripillan J, Nicholson B. Gap-junction channels dysfunction in deafness and hearing loss. Antioxid Redox Signal. 2009; 11:309-322. [PubMed: 18837651]

419. Martínez-Ruiz A, Lamas S. S-nitrosylation: A potential new paradigm in signal transduction. Cardiovasc Res. 2004; 62:43-52. [PubMed: 15023551]

420. Massa PT, Mugnaini E. Cell junctions and intramembrane particles of astrocytes and oligodendrocytes: A freeze-fracture study. Neuroscience. 1982; 7:523-538. [PubMed: 7078735]

421. Matesic D, Tillen T, Sitaramayya A. Connexin 40 expression in bovine and rat retinas. Cell Biol Int. 2003; 27:89-99. [PubMed: 12662966]

422. Mathias RT, Kistler J, Donaldson P. The lens circulation. J Membr Biol. 2007; 216:1-16. [PubMed: 17568975]

423. Mathias RT, Rae JL, Eisenberg RS. The lens as a nonuniform spherical syncytium. Biophys J. 1981; 34:61-83. [PubMed: 7213932]

424. Mathias RT, Riquelme G, Rae JL. Cell to cell communication and $\mathrm{pH}$ in the frog lens. J Gen Physiol. 1991; 98:1085-1103. [PubMed: 1783895]

425. Mathias RT, White TW, Gong X. Lens gap junctions in growth, differentiation, and homeostasis. Physiol Rev. 2010; 90:179-206. [PubMed: 20086076]

426. Maurer P, Weingart R. Cell pairs isolated from adult guinea pig and rat hearts: Effects of [Ca2+]i on nexal membrane resistance. Pflugers Arch. 1987; 409:394-402. [PubMed: 3627957]

427. McCulloch F, Chambrey R, Eladari D, Peti-Peterdi J. Localization of connexin 30 in the luminal membrane of cells in the distal nephron. Am J Physiol Renal Physiol. 2005; 289:F1304-F1312. [PubMed: 16077080]

428. Meda P. Gap junction involvement in secretion: The pancreas experience. Clin Exp Pharmacol Physiol. 1996; 23:1053-1057. [PubMed: 8977159]

429. Mehta PP, Bertram JS, Loewenstein WR. Growth inhibition of transformed cells correlates with their junctional communication with normal cells. Cell. 1986; 44:187-196. [PubMed: 2416473]

430. Mehta PP, Perez-Stable C, Nadji M, Mian M, Asotra K, Roos BA. Suppression of human prostate cancer cell growth by forced expression of connexin genes. Dev Genet. 1999; 24:91-110. [PubMed: 10079514]

431. Menichella DM, Goodenough DA, Sirkowski E, Scherer SS, Paul DL. Connexins are critical for normal myelination in the CNS. J Neurosci. 2003; 23:5963-5973. [PubMed: 12843301]

432. Mesnil M, Crespin S, Avanzo JL, Zaidan-Dagli ML. Defective gap junctional intercellular communication in the carcinogenic process. Biochim Biophys Acta. 2005; 1719:125-145. [PubMed: 16359943]

433. Mesnil M, Krutovskikh V, Piccoli C, Elfgang C, Traub O, Willecke K, Yamasaki H. Negative growth control of HeLa cells by connexin genes: Connexin species specificity. Cancer Res. 1995; 55:629-639. [PubMed: 7834634]

434. Meyers JR, MacDonald RB, Duggan A, Lenzi D, Standaert DG, Corwin JT, Corey DP. Lighting up the senses: FM1-43 loading of sensory cells through nonselective ion channels. J Neurosci. 2003; 23:4054-4065. [PubMed: 12764092] 
435. Miller T, Dahl G, Werner R. Structure of a gap junction gene: Rat connexin-32. Biosci Rep. 1988; 8:455-464. [PubMed: 2852976]

436. Miller TM, Goodenough DA. Evidence for two physiologically distinct gap junctions expressed by the chick lens epithelial cell. J Cell Biol. 1986; 102:194-199. [PubMed: 3079768]

437. Miro-Casas E, Ruiz-Meana M, Agullo E, Stahlhofen S, Rodriguez-Sinovas A, Cabestrero A, Jorge I, Torre I, Vazquez J, Boengler K, Schulz R, Heusch G, Garcia-Dorado D. Connexin43 in cardiomyocyte mitochondria contributes to mitochondrial potassium uptake. Cardiovasc Res. 2009; 83:747-756. [PubMed: 19460776]

438. Mitchell JA, Lye SJ. Differential expression of activator protein-1 transcription factors in pregnant rat myometrium. Biol Reprod. 2002; 67:240-246. [PubMed: 12080023]

439. Miura T, Miki T, Yano T. Role of the gap junction in ischemic preconditioning in the heart. Am $\mathbf{J}$ Physiol Heart Circ Physiol. 2010; 298:H1115-H1125. [PubMed: 20118409]

440. Miyoshi H, Boyle MB, MacKay LB, Garfield RE. Voltage-clamp studies of gap junctions between uterine muscle cells during term and preterm labor. Biophys J. 1996; 71:1324-1334. [PubMed: 8874006]

441. Mohammad G, Kowluru RA. Novel role of mitochondrial matrix metalloproteinase- 2 in the development of diabetic retinopathy. Invest Ophthalmol Vis Sci. 2011; 52:3832-3841. [PubMed: 21345984]

442. Mollerup S, Hofgaard JP, Braunstein TH, Kjenseth A, Leithe E, Rivedal E, Holstein-Rathlou NH, Nielsen MS. Norepinephrine inhibits intercellular coupling in rat cardiomyocytes by ubiquitination of connexin43 gap junctions. Cell Commun Adhes. 2011; 18:57-65. [PubMed: 21936631]

443. Monaghan P, Perusinghe N, Carlile G, Evans WH. Rapid modulation of gap junction expression in mouse mammary gland during pregnancy, lactation, and involution. J Histochem Cytochem. 1994; 42:931-938. [PubMed: 8014476]

444. Montecino-Rodriguez E, Dorshkind K. Regulation of hematopoiesis by gap junction-mediated intercellular communication. J Leukoc Biol. 2001; 70:341-347. [PubMed: 11527982]

445. Montecino-Rodriguez E, Leathers H, Dorshkind K. Expression of connexin 43 (Cx43) is critical for normal hematopoiesis. Blood. 2000; 96:917-924. [PubMed: 10910905]

446. Moorby C, Patel M. Dual functions for connexins: Cx43 regulates growth independently of gap junction formation. Exp Cell Res. 2001; 271:238-248. [PubMed: 11716536]

447. Morel S, Burnier L, Kwak B. Connexins participate in the initiation and progression of atherosclerosis. Semin Immunopathol. 2009; 31:49-61. [PubMed: 19404645]

448. Moreno AP. Biophysical properties of homomeric and heteromultimeric channels formed by cardiac connexins. Cardiovasc Res. 2004; 62:276-286. [PubMed: 15094348]

449. Moreno AP, Chanson M, Elenes S, Anumonwo J, Scerri I, Gu H, Taffet SM, Delmar M. Role of the carboxyl terminal of connexin43 in transjunctional fast voltage gating. Circ Res. 2002; 90:450-457. [PubMed: 11884375]

450. Moreno AP, Fishman GI, Spray DC. Phosphorylation shifts unitary conductance and modifies voltage dependent kinetics of human connexin43 gap junction channels. Biophys J. 1992; 62:5153. [PubMed: 1376174]

451. Moreno AP, Rook MB, Fishman GI, Spray DC. Gap junction channels: Distinct voltage-sensitive and -insensitive conductance states. Biophys J. 1994; 67:113-119. [PubMed: 7522596]

452. Moreno AP, Sáez JC, Fishman GI, Spray DC. Human Connexin43 gap junction channels. Regulation of unitary conductances by phosphorylation. Circ Res. 1994; 74:1050-1057. [PubMed: 7514508]

453. Mori Y, Otabe S, Dina C, Yasuda K, Populaire C, Lecoeur C, Vatin V, Durand E, Hara K, Okada T, Tobe K, Boutin P, Kadowaki T, Froguel P. Genome-wide search for type 2 diabetes in Japanese affected sib-pairs confirms susceptibility genes on $3 q, 15 q$, and $20 q$ and identifies two new candidate Loci on 7p and 11p. Diabetes. 2002; 51:1247-1255. [PubMed: 11916952]

454. Morley GE, Taffet SM, Delmar M. Intramolecular interactions mediate $\mathrm{pH}$ regulation of connexin43 channels. Biophys J. 1996; 70:1294-1302. [PubMed: 8785285] 
455. Morley GE, Vaidya D, Samie FH, Lo C, Delmar M, Jalife J. Characterization of conduction in the ventricles of normal and heterozygous Cx43 knockout mice using optical mapping. J Cardiovasc Electrophysiol. 1999; 10:1361-1375. [PubMed: 10515561]

456. Muller DJ, Hand GM, Engel A, Sosinsky GE. Conformational changes in surface structures of isolated connexin 26 gap junctions. Embo J. 2002; 21:3598-3607. [PubMed: 12110573]

457. Muramatsu T, Hashimoto S, Shimono M. Differential expression of gap junction proteins connexin32 and 43 in rat submandibular and sublingual glands. J Histochem Cytochem. 1996; 44:49-56. [PubMed: 8543782]

458. Murata Y, Yasuo T, Yoshida R, Obata K, Yanagawa Y, Margolskee RF, Ninomiya Y. Action potential-enhanced ATP release from taste cells through hemichannels. J Neurophysiol. 2010; 104:896-901. [PubMed: 20519578]

459. Murry CE, Jennings RB, Reimer KA. Preconditioning with ischemia: A delay of lethal cell injury in ischemic myocardium. Circulation. 1986; 74:1124-1136. [PubMed: 3769170]

460. Musil, LS. Biogenesis and Degradation of Gap Junctions. In: Harris, AL.; Locke, D., editors. Connexins. A Guide. Humana Press; New York: 2009. p. 225-240.

461. Musil LS, Cunningham BA, Edelman GM, Goodenough DA. Differential phosphorylation of the gap junction protein connexin43 in junctional communication-competent and -deficient cell lines. J Cell Biol. 1990; 111:2077-2088. [PubMed: 2172261]

462. Musil LS, Goodenough DA. Biochemical analysis of connexin43 intracellular transport, phosphorylation, and assembly into gap junctional plaques. J Cell Biol. 1991; 115:1357-1374. [PubMed: 1659577]

463. Musil LS, Goodenough DA. Multisubunit assembly of an integral plasma membrane channel protein, gap junction connexin43, occurs after exit from the ER. Cell. 1993; 74:1065-1077. [PubMed: 7691412]

464. Musil LS, Le AC, VanSlyke JK, Roberts LM. Regulation of connexin degradation as a mechanism to increase gap junction assembly and function. J Biol Chem. 2000; 275:2520725215. [PubMed: 10940315]

465. Nagy JI, Li X, Rempel J, Stelmack G, Patel D, Staines WA, Yasumura T, Rash JE. Connexin26 in adult rodent central nervous system: Demonstration at astrocytic gap junctions and colocalization with connexin30 and connexin43. J Comp Neurol. 2001; 441:302-323. [PubMed: 11745652]

466. Nagy JI, Rash JE. Connexins and gap junctions of astrocytes and oligodendrocytes in the CNS. Brain Res Brain Res Rev. 2000; 32:29-44. [PubMed: 10751655]

467. Nakase T, Sohl G, Theis M, Willecke K, Naus CC. Increased apoptosis and inflammation after focal brain ischemia in mice lacking connexin43 in astrocytes. Am J Pathol. 2004; 164:20672075. [PubMed: 15161641]

468. Naus CC, Hearn S, Zhu D, Nicholson BJ, Shivers RR. Ultrastructural analysis of gap junctions in C6 glioma cells transfected with connexin43 cDNA. Exp Cell Res. 1993; 206:72-84. [PubMed: 8387023]

469. Neijssen J, Herberts C, Drijfhout JW, Reits E, Janssen L, Neefjes J. Cross-presentation by intercellular peptide transfer through gap junctions. Nature. 2005; 434:83-88. [PubMed: 15744304]

470. Neijssen J, Pang B, Neefjes J. Gap junction-mediated intercellular communication in the immune system. Prog Biophys Mol Biol. 2007; 94:207-218. [PubMed: 17467043]

471. Nelles E, Butzler C, Jung D, Temme A, Gabriel HD, Dahl U, Traub O, Stumpel F, Jungermann K, Zielasek J, Toyka KV, Dermietzel R, Willecke K. Defective propagation of signals generated by sympathetic nerve stimulation in the liver of connexin32-deficient mice. Proc Natl Acad Sci U S A. 1996; 93:9565-9570. [PubMed: 8790370]

472. Nemeth L, Maddur S, Puri P. Immunolocalization of the gap junction protein Connexin43 in the interstitial cells of Cajal in the normal and Hirschsprung's disease bowel. J Pediatr Surg. 2000; 35:823-828. [PubMed: 10873019]

473. Nicholson B, Dermietzel R, Teplow D, Traub O, Willecke K, Revel JP. Two homologous protein components of hepatic gap junctions. Nature. 1987; 329:732-734. [PubMed: 2823143] 
474. Nickel BM, DeFranco BH, Gay VL, Murray SA. Clathrin and Cx43 gap junction plaque endoexocytosis. Biochem Biophys Res Commun. 2008; 374:679-682. [PubMed: 18675253]

475. Niessen H, Willecke K. Strongly decreased gap junctional permeability to inositol 1,4, 5trisphosphate in connexin32 deficient hepatocytes. FEBS Lett. 2000; 466:112-114. [PubMed: 10648823]

476. Nlend RN, Michon L, Bavamian S, Boucard N, Caille D, Cancela J, Charollais A, Charpantier E, Klee P, Peyrou M, Populaire C, Zulianello L, Meda P. Connexin36 and pancreatic beta-cell functions. Arch Physiol Biochem. 2006; 112:74-81. [PubMed: 16931449]

477. Noma A, Tsuboi N. Dependence of junctional conductance on proton, calcium and magnesium ions in cardiac paired cells of guinea-pig. J Physiol. 1987; 382:193-211. [PubMed: 2442361]

478. Norris RP, Ratzan WJ, Freudzon M, Mehlmann LM, Krall J, Movsesian MA, Wang H, Ke H, Nikolaev VO, Jaffe LA. Cyclic GMP from the surrounding somatic cells regulates cyclic AMP and meiosis in the mouse oocyte. Development. 2009; 136:1869-1878. [PubMed: 19429786]

479. O'Quinn MP, Palatinus JA, Harris BS, Hewett KW, Gourdie RG. A peptide mimetic of the connexin43 carboxyl terminus reduces gap junction remodeling and induced arrhythmia following ventricular injury. Circ Res. 2011; 108:704-715. [PubMed: 21273554]

480. Odermatt B, Wellershaus K, Wallraff A, Seifert G, Degen J, Euwens C, Fuss B, Bussow H, Schilling K, Steinhauser C, Willecke K. Connexin 47 (Cx47)-deficient mice with enhanced green fluorescent protein reporter gene reveal predominant oligodendrocytic expression of $\mathrm{Cx} 47$ and display vacuolized myelin in the CNS. J Neurosci. 2003; 23:4549-4559. [PubMed: 12805295]

481. Ogawa T, Hayashi T, Kyoizumi S, Ito T, Trosko JE, Yorioka N. Upregulation of gap junctional intercellular communication by hexamethylene bisacetamide in cultured human peritoneal mesothelial cells. Lab Invest. 1999; 79:1511-1520. [PubMed: 10616202]

482. Oh S, Ri Y, Bennett MV, Trexler EB, Verselis VK, Bargiello TA. Changes in permeability caused by connexin 32 mutations underlie X-linked Charcot-Marie-Tooth disease. Neuron. 1997; 19:927-938. [PubMed: 9354338]

483. Oike M, Droogmans G, Ito Y. [ATP release pathways in vascular endothelial cells]. Nippon Yakurigaku Zasshi. 2004; 123:403-411. [PubMed: 15170080]

484. Olbina G, Eckhart W. Mutations in the second extracellular region of connexin 43 prevent localization to the plasma membrane, but do not affect its ability to suppress cell growth. Mol Cancer Res. 2003; 1:690-700. [PubMed: 12861055]

485. Omori Y, Yamasaki H. Mutated connexin43 proteins inhibit rat glioma cell growth suppression mediated by wild-type connexin43 in a dominant-negative manner. Int J Cancer. 1998; 78:446453. [PubMed: 9797133]

486. Oosthoek PW, Viragh S, Lamers WH, Moorman AF. Immunohistochemical delineation of the conduction system. II: The atrioventricular node and Purkinje fibers. Circ Res. 1993; 73:482491. [PubMed: 8394224]

487. Oosthoek PW, Viragh S, Mayen AE, van Kempen MJ, Lamers WH, Moorman AF. Immunohistochemical delineation of the conduction system. I: The sinoatrial node. Circ Res. 1993; 73:473-481. [PubMed: 8394223]

488. Orellana JA, Hernandez DE, Ezan P, Velarde V, Bennett MV, Giaume C, Saez JC. Hypoxia in high glucose followed by reoxygenation in normal glucose reduces the viability of cortical astrocytes through increased permeability of connexin 43 hemichannels. Glia. 2010; 58:329-343. [PubMed: 19705457]

489. Orthmann-Murphy JL, Abrams CK, Scherer SS. Gap junctions couple astrocytes and oligodendrocytes. J Mol Neurosci. 2008; 35:101-116. [PubMed: 18236012]

490. Oshima A, Tani K, Hiroaki Y, Fujiyoshi Y, Sosinsky GE. Three-dimensional structure of a human connexin26 gap junction channel reveals a plug in the vestibule. Proc Natl Acad Sci U S A. 2007; 104:10034-10039. [PubMed: 17551008]

491. Ou CW, Orsino A, Lye SJ. Expression of connexin-43 and connexin-26 in the rat myometrium during pregnancy and labor is differentially regulated by mechanical and hormonal signals. Endocrinology. 1997; 138:5398-5407. [PubMed: 9389525]

492. Oviedo-Orta E, Errington RJ, Evans WH. Gap junction intercellular communication during lymphocyte transendothelial migration. Cell Biol Int. 2002; 26:253-263. [PubMed: 11991653] 
493. Oviedo-Orta E, Gasque P, Evans WH. Immunoglobulin and cytokine expression in mixed lymphocyte cultures is reduced by disruption of gap junction intercellular communication. FASEB J. 2001; 15:768-774. [PubMed: 11259395]

494. Oviedo-Orta E, Perreau M, Evans WH, Potolicchio I. Control of the proliferation of activated CD4+ T cells by connexins. J Leukoc Biol. 2010; 88:79-86. [PubMed: 20233983]

495. Owens DF, Kriegstein AR. Patterns of intracellular calcium fluctuation in precursor cells of the neocortical ventricular zone. J Neurosci. 1998; 18:5374-5388. [PubMed: 9651220]

496. Oyamada M, Oyamada Y, Takamatsu T. Regulation of connexin expression. Biochim Biophys Acta. 2005; 1719:6-23. [PubMed: 16359940]

497. Pain T, Yang XM, Critz SD, Yue Y, Nakano A, Liu GS, Heusch G, Cohen MV, Downey JM. Opening of mitochondrial K(ATP) channels triggers the preconditioned state by generating free radicals. Circ Res. 2000; 87:460-466. [PubMed: 10988237]

498. Palacios-Prado N, Bukauskas FF. Heterotypic gap junction channels as voltage-sensitive valves for intercellular signaling. Proc Natl Acad Sci U S A. 2009; 106:14855-14860. [PubMed: 19706392]

499. Palacios-Prado N, Sonntag S, Skeberdis VA, Willecke K, Bukauskas FF. Gating, permselectivity and $\mathrm{pH}$-dependent modulation of channels formed by connexin57, a major connexin of horizontal cells in the mouse retina. J Physiol. 2009; 587:3251-3269. [PubMed: 19433576]

500. Panchin Y, Kelmanson I, Matz M, Lukyanov K, Usman N, Lukyanov S. A ubiquitous family of putative gap junction molecules. Curr Biol. 2000; 10:R473-R474. [PubMed: 10898987]

501. Panchin YV. Evolution of gap junction proteins-the pannexin alternative. J Exp Biol. 2005; 208:1415-1419. [PubMed: 15802665]

502. Pang B, Neijssen J, Qiao X, Janssen L, Janssen H, Lippuner C, Neefjes J. Direct antigen presentation and gap junction mediated cross-presentation during apoptosis. J Immunol. 2009; 183:1083-1090. [PubMed: 19553546]

503. Pankratov Y, Lalo U, Verkhratsky A, North RA. Vesicular release of ATP at central synapses. Pflugers Arch. 2006; 452:589-597. [PubMed: 16639550]

504. Paul DL, Ebihara L, Takemoto LJ, Swenson KI, Goodenough DA. Connexin46, a novel lens gap junction protein, induces voltage-gated currents in nonjunctional plasma membrane of Xenopus oocytes. J Cell Biol. 1991; 115:1077-1089. [PubMed: 1659572]

505. Paznekas WA, Karczeski B, Vermeer S, Lowry RB, Delatycki M, Laurence F, Koivisto PA, Van ML, Boyadjiev SA, Bodurtha JN, Jabs EW. GJA1 mutations, variants, and connexin 43 dysfunction as it relates to the oculodentodigital dysplasia phenotype. Hum Mutat. 2009; 30:724733. [PubMed: 19338053]

506. Pearson RA, Dale N, Llaudet E, Mobbs P. ATP released via gap junction hemichannels from the pigment epithelium regulates neural retinal progenitor proliferation. Neuron. 2005; 46:731-744. [PubMed: 15924860]

507. Penuela S, Bhalla R, Gong XQ, Cowan KN, Celetti SJ, Cowan BJ, Bai D, Shao Q, Laird DW. Pannexin 1 and pannexin 3 are glycoproteins that exhibit many distinct characteristics from the connexin family of gap junction proteins. J Cell Sci. 2007; 120:3772-3783. [PubMed: 17925379]

508. Peracchia C. Chemical gating of gap junction channels; roles of calcium, $\mathrm{pH}$ and calmodulin. Biochim Biophys Acta. 2004; 1662:61-80. [PubMed: 15033579]

509. Peracchia C, Sotkis A, Wang XG, Peracchia LL, Persechini A. Calmodulin directly gates gap junction channels. J Biol Chem. 2000; 275:26220-26224. [PubMed: 10852921]

510. Peracchia C, Young KC, Wang XG, Peracchia LL. Is the voltage gate of connexins CO2sensitive? Cx45 channels and inhibition of calmodulin expression. J Membr Biol. 2003; 195:5362. [PubMed: 14502426]

511. Perez-Armendariz EM, Romano MC, Luna J, Miranda C, Bennett MV, Moreno AP. Characterization of gap junctions between pairs of Leydig cells from mouse testis. Am J Physiol. 1994; 267:C570-C580. [PubMed: 7521131]

512. Perez-Armendariz M, Roy C, Spray DC, Bennett MV. Biophysical properties of gap junctions between freshly dispersed pairs of mouse pancreatic beta cells. Biophys J. 1991; 59:76-92. [PubMed: 2015391] 
513. Petrocelli T, Lye SJ. Regulation of transcripts encoding the myometrial gap junction protein, connexin-43, by estrogen and progesterone. Endocrinology. 1993; 133:284-290. [PubMed: 8391423]

514. Pfahnl A, Dahl G. Gating of cx46 gap junction hemichannels by calcium and voltage. Pflugers Arch. 1999; 437:345-353. [PubMed: 9914390]

515. Pfeifer I, Anderson C, Werner R, Oltra E. Redefining the structure of the mouse connexin43 gene: Selective promoter usage and alternative splicing mechanisms yield transcripts with different translational efficiencies. Nucleic Acids Res. 2004; 32:4550-4562. [PubMed: 15328367]

516. Pfeifer U. Autophagic sequestration of internalized gap junctions in rat liver. Eur J Cell Biol. 1980; 21:244-246. [PubMed: 7449766]

517. Phelan P. Innexins: Members of an evolutionarily conserved family of gap-junction proteins. Biochim Biophys Acta. 2005; 1711:225-245. [PubMed: 15921654]

518. Piatnitski Chekler EL, Butera JA, Di L, Swillo RE, Morgan GA, Rossman EI, Huselton C, Larsen BD, Hennan JK. Discovery of a class of potent gap-junction modifiers as novel antiarrhythmic agents. Bioorg Med Chem Lett. 2009; 19:4551-4554. [PubMed: 19616941]

519. Piechocki MP, Burk RD, Ruch RJ. Regulation of connexin 32 and connexin 43 gene expression by DNA methylation in rat liver cells. Carcinogenesis. 1999; 20:401-406. [PubMed: 10190553]

520. Piehl M, Lehmann C, Gumpert A, Denizot JP, Segretain D, Falk MM. Internalization of large double-membrane intercellular vesicles by a clathrin-dependent endocytic process. Mol Biol Cell. 2007; 18:337-347. [PubMed: 17108328]

521. Piersanti M, Lye SJ. Increase in messenger ribonucleic acid encoding the myometrial gap junction protein, connexin-43, requires protein synthesis and is associated with increased expression of the activator protein-1, c-fos. Endocrinology. 1995; 136:3571-3578. [PubMed: 7628395]

522. Plotkin LI, Manolagas SC, Bellido T. Transduction of cell survival signals by connexin-43 hemichannels. J Biol Chem. 2002; 277:8648-8657. [PubMed: 11741942]

523. Pluciennik F, Verrecchia F, Bastide B, Herve JC, Joffre M, Deleze J. Reversible interruption of gap junctional communication by testosterone propionate in cultured Sertoli cells and cardiac myocytes. J Membr Biol. 1996; 149:169-177. [PubMed: 8801349]

524. Plum A, Hallas G, Magin T, Dombrowski F, Hagendorff A, Schumacher B, Wolpert C, Kim J, Lamers WH, Evert M, Meda P, Traub O, Willecke K. Unique and shared functions of different connexins in mice. Curr Biol. 2000; 10:1083-1091. [PubMed: 10996788]

525. Pointis G, Fiorini C, Defamie N, Segretain D. Gap junctional communication in the male reproductive system. Biochim Biophys Acta. 2005; 1719:102-116. [PubMed: 16259941]

526. Pointis G, Gilleron J, Carette D, Segretain D. Physiological and physiopathological aspects of connexins and communicating gap junctions in spermatogenesis. Philos Trans R Soc Lond B Biol Sci. 2010; 365:1607-1620. [PubMed: 20403873]

527. Postma FR, Hengeveld T, Alblas J, Giepmans BNG, Zondag GCM, Jalink K, Moolenaar WH. Acute loss of cell-cell communication caused by G protein-coupled receptors: A critical role for c-Src. J Cell Biol. 1998; 140:1199-1209. [PubMed: 9490732]

528. Presley CA, Lee AW, Kastl B, Igbinosa I, Yamada Y, Fishman GI, Gutstein DE, Cancelas JA. Bone marrow connexin-43 expression is critical for hematopoietic regeneration after chemotherapy. Cell Commun Adhes. 2005; 12:307-317. [PubMed: 16531325]

529. Princen F, Robe P, Gros D, Jarry-Guichard T, Gielen J, Merville MP, Bours V. Rat gap junction connexin-30 inhibits proliferation of glioma cell lines. Carcinogenesis. 2001; 22:507-513. [PubMed: 11238193]

530. Procida K, Jørgensen L, Schmitt N, Delmar M, Taffet SM, Holstein-Rathlou NH, Nielsen MS, Braunstein TH. Phosphorylation of connexin 43 on serine 306 regulates electrical coupling. Heart Rhythm. 2009; 6:1632-1638. [PubMed: 19879542]

531. Puljung MC, Berthoud VM, Beyer EC, Hanck DA. Polyvalent cations constitute the voltage gating particle in human connexin37 hemichannels. J Gen Physiol. 2004; 124:587-603. [PubMed: 15504903] 
532. Puranam KL, Laird DW, Revel JP. Trapping an intermediate form of connexin43 in the Golgi. Exp Cell Res. 1993; 206:85-92. [PubMed: 8387024]

533. Puri CP, Garfield RE. Changes in hormone levels and gap junctions in the rat uterus during pregnancy and parturition. Biol Reprod. 1982; 27:967-975. [PubMed: 6959654]

534. Qin H, Shao Q, Curtis H, Galipeau J, Belliveau DJ, Wang T, Alaoui-Jamali MA, Laird DW. Retroviral delivery of connexin genes to human breast tumor cells inhibits in vivo tumor growth by a mechanism that is independent of significant gap junctional intercellular communication. $\mathrm{J}$ Biol Chem. 2002; 277:29132-29138. [PubMed: 12042301]

535. Qin H, Shao Q, Igdoura SA, Alaoui-Jamali MA, Laird DW. Lysosomal and proteasomal degradation play distinct roles in the life cycle of $\mathrm{Cx} 43$ in gap junctional intercellular communication-deficient and -competent breast tumor cells. J Biol Chem. 2003; 278:3000530014. [PubMed: 12767974]

536. Qiu C, Coutinho P, Frank S, Franke S, Law LY, Martin P, Green CR, Becker DL. Targeting connexin43 expression accelerates the rate of wound repair. Curr Biol. 2003; 13:1697-1703. [PubMed: 14521835]

537. Qu C, Gardner P, Schrijver I. The role of the cytoskeleton in the formation of gap junctions by Connexin 30. Exp Cell Res. 2009; 315:1683-1692. [PubMed: 19285977]

538. Quan XQ, Bai R, Lu JG, Patel C, Liu N, Ruan Y, Chen BD, Ruan L, Zhang CT. Pharmacological enhancement of cardiac gap junction coupling prevents arrhythmias in canine LQT2 model. Cell Commun Adhes. 2009; 16:29-38. [PubMed: 19629804]

539. Rae JL, Bartling C, Rae J, Mathias RT. Dye transfer between cells of the lens. J Membr Biol. 1996; 150:89-103. [PubMed: 8699483]

540. Rahman S, Carlile G, Evans WH. Assembly of hepatic gap junctions. Topography and distribution of connexin 32 in intracellular and plasma membranes determined using sequencespecific antibodies. J Biol Chem. 1993; 268:1260-1265. [PubMed: 8380409]

541. Rahman S, Evans WH. Topography of connexin32 in rat liver gap junctions. Evidence for an intramolecular disulphide linkage connecting the two extracellular peptide loops. J Cell Sci. 1991; 100(Pt 3):567-578. [PubMed: 1667015]

542. Rana S, Dringen R. Gap junction hemichannel-mediated release of glutathione from cultured rat astrocytes. Neurosci Lett. 2007; 415:45-48. [PubMed: 17222973]

543. Rash JE. Molecular disruptions of the panglial syncytium block potassium siphoning and axonal saltatory conduction: Pertinence to neuromyelitis optica and other demyelinating diseases of the central nervous system. Neuroscience. 2010; 168:982-1008. [PubMed: 19850107]

544. Rash JE, Davidson KG, Kamasawa N, Yasumura T, Kamasawa M, Zhang C, Michaels R, Restrepo D, Ottersen OP, Olson CO, Nagy JI. Ultrastructural localization of connexins (Cx36, $\mathrm{Cx} 43, \mathrm{Cx} 45)$, glutamate receptors and aquaporin-4 in rodent olfactory mucosa, olfactory nerve and olfactory bulb. J Neurocytol. 2005; 34:307-341. [PubMed: 16841170]

545. Rash JE, Staehelin LA, Ellisman MH. Rectangular arrays of particles on freeze-cleaved plasma membranes are not gap junctions. Exp Cell Res. 1974; 86:187-190. [PubMed: 4831157]

546. Rash JE, Yasumura T, Davidson KG, Furman CS, Dudek FE, Nagy JI. Identification of cells expressing $\mathrm{Cx} 43, \mathrm{Cx} 30, \mathrm{Cx} 26, \mathrm{Cx} 32$ and $\mathrm{Cx} 36$ in gap junctions of rat brain and spinal cord. Cell Commun Adhes. 2001; 8:315-320. [PubMed: 12064610]

547. Rash JE, Yasumura T, Dudek FE, Nagy JI. Cell-specific expression of connexins and evidence of restricted gap junctional coupling between glial cells and between neurons. J Neurosci. 2001; 21:1983-2000. [PubMed: 11245683]

548. Ravier MA, Guldenagel M, Charollais A, Gjinovci A, Caille D, Sohl G, Wollheim CB, Willecke $\mathrm{K}$, Henquin JC, Meda P. Loss of connexin36 channels alters beta-cell coupling, islet synchronization of glucose-induced $\mathrm{Ca} 2+$ and insulin oscillations, and basal insulin release. Diabetes. 2005; 54:1798-1807. [PubMed: 15919802]

549. Reaume AG, De Sousa PA, Kulkarni S, Langille BL, Zhu D, Davies TC, Juneja SC, Kidder GM, Rossant J. Cardiac malformation in neonatal mice lacking connexin43. Science. 1995; 267:18311834. [PubMed: 7892609] 
550. Remo BF, Qu J, Volpicelli FM, Giovannone S, Shin D, Lader J, Liu FY, Zhang J, Lent DS, Morley GE, Fishman GI. Phosphatase-resistant gap junctions inhibit pathological remodeling and prevent arrhythmias. Circ Res. 2011; 108:1459-1466. [PubMed: 21527737]

551. Renthal NE, Chen CC, Williams KC, Gerard RD, Prange-Kiel J, Mendelson CR. miR-200 family and targets, ZEB1 and ZEB2, modulate uterine quiescence and contractility during pregnancy and labor. Proc Natl Acad Sci U S A. 2010; 107:20828-20833. [PubMed: 21079000]

552. Retamal MA, Cortes CJ, Reuss L, Bennett MV, Saez JC. S-nitrosylation and permeation through connexin 43 hemichannels in astrocytes: Induction by oxidant stress and reversal by reducing agents. Proc Natl Acad Sci U S A. 2006; 103:4475-4480. [PubMed: 16537412]

553. Retamal MA, Froger N, Palacios-Prado N, Ezan P, Saez PJ, Saez JC, Giaume C. Cx43 hemichannels and gap junction channels in astrocytes are regulated oppositely by proinflammatory cytokines released from activated microglia. J Neurosci. 2007; 27:1378113792. [PubMed: 18077690]

554. Retamal MA, Schalper KA, Shoji KF, Bennett MV, Saez JC. Opening of connexin 43 hemichannels is increased by lowering intracellular redox potential. Proc Natl Acad Sci U S A. 2007; 104:8322-8327. [PubMed: 17494739]

555. Retamal MA, Schalper KA, Shoji KF, Orellana JA, Bennett MV, Saez JC. Possible involvement of different connexin43 domains in plasma membrane permeabilization induced by ischemiareperfusion. J Membr Biol. 2007; 218:49-63. [PubMed: 17705051]

556. Retamal MA, Cortés CJ, Reuss L, Bennett MVL, Sáez JC. Snitrosylation and permeation through connexin 43 hemichannels in astrocytes: Induction by oxidant stress and reversal by reducing agents. Proc Natl Acad Sci U S A. 2006; 103:4475-4480. [PubMed: 16537412]

557. Retamal MA, Yin S, Altenberg GA, Reuss L. Modulation of Cx46 hemichannels by nitric oxide. Am J Physiol Cell Physiol. 2009; 296:C1356-C1363. [PubMed: 19357237]

558. Revilla A, Bennett MV, Barrio LC. Molecular determinants of membrane potential dependence in vertebrate gap junction channels. Proc Natl Acad Sci U S A. 2000; 97:14760-14765. [PubMed: 11121075]

559. Revilla A, Castro C, Barrio LC. Molecular dissection of transjunctional voltage dependence in the connexin-32 and connexin-43 junctions. Biophys J. 1999; 77:1374-1383. [PubMed: 10465749]

560. Rios M, Hermoso M, Sanchez TM, Croxatto HB, Villalon MJ. Effect of oestradiol and progesterone on the instant and directional velocity of microsphere movements in the rat oviduct: Gap junctions mediate the kinetic effect of oestradiol. Reprod Fertil Dev. 2007; 19:634-640. [PubMed: 17601411]

561. Ripps H, Qian H, Zakevicius J. Pharmacological enhancement of hemi-gap-junctional currents in Xenopus oocytes. J Neurosci Methods. 2002; 121:81-92. [PubMed: 12393164]

562. Ripps H, Qian H, Zakevicius J. Properties of connexin26 hemichannels expressed in Xenopus oocytes. Cell Mol Neurobiol. 2004; 24:647-665. [PubMed: 15485136]

563. Risek B, Guthrie S, Kumar N, Gilula NB. Modulation of gap junction transcript and protein expression during pregnancy in the rat. J Cell Biol. 1990; 110:269-282. [PubMed: 1688855]

564. Risley MS, Tan IP, Roy C, Saez JC. Cell-, age- and stage-dependent distribution of connexin43 gap junctions in testes. J Cell Sci. 1992; 103(Pt 1):81-96. [PubMed: 1331136]

565. Rivedal E, Opsahl H. Role of PKC and MAP kinase in EGF- and TPA-induced connexin43 phosphorylation and inhibition of gap junction intercellular communication in rat liver epithelial cells. Carcinogenesis. 2001; 22:1543-1550. [PubMed: 11532878]

566. Robb-Gaspers LD, Thomas AP. Coordination of Ca2+ signaling by intercellular propagation of Ca2+ waves in the intact liver. J Biol Chem. 1995; 270:8102-8107. [PubMed: 7713913]

567. Rodriguez-Sinovas A, Boengler K, Cabestrero A, Gres P, Morente M, Ruiz-Meana M, Konietzka I, Miro E, Totzeck A, Heusch G, Schulz R, Garcia-Dorado D. Translocation of connexin 43 to the inner mitochondrial membrane of cardiomyocytes through the heat shock protein 90dependent TOM pathway and its importance for cardioprotection. Circ Res. 2006; 99:93-101. [PubMed: 16741159]

568. Romanello M, Pani B, Bicego M, D’Andrea P. Mechanically induced ATP release from human osteoblastic cells. Biochem Biophys Res Commun. 2001; 289:1275-1281. [PubMed: 11741333] 
569. Rong P, Wang X, Niesman I, Wu Y, Benedetti LE, Dunia I, Levy E, Gong X. Disruption of Gja8 (alpha8 connexin) in mice leads to microphthalmia associated with retardation of lens growth and lens fiber maturation. Development. 2002; 129:167-174. [PubMed: 11782410]

570. Roscoe WA, Barr KJ, Mhawi AA, Pomerantz DK, Kidder GM. Failure of spermatogenesis in mice lacking connexin43. Biol Reprod. 2001; 65:829-838. [PubMed: 11514348]

571. Rossman EI, Liu K, Morgan GA, Swillo RE, Krueger JA, Gardell SJ, Butera J, Gruver M, Kantrowitz J, Feldman HS, Petersen JS, Haugan K, Hennan JK. The gap junction modifier, GAP-134 [(2S,4R)-1-(2-aminoacetyl)-4-benzamido-pyrrolidine-2-carboxylic acid], improves conduction and reduces atrial fibrillation/flutter in the canine sterile pericarditis model. $\mathrm{J}$ Pharmacol Exp Ther. 2009; 329:1127-1133. [PubMed: 19252062]

572. Rottingen J, Iversen JG. Ruled by waves? Intracellular and intercellular calcium signalling. Acta Physiol Scand. 2000; 169:203-219. [PubMed: 10886035]

573. Rottlaender D, Boengler K, Wolny M, Michels G, Endres-Becker J, Motloch LJ, Schwaiger A, Buechert A, Schulz R, Heusch G, Hoppe UC. Connexin 43 acts as a cytoprotective mediator of signal transduction by stimulating mitochondrial KATP channels in mouse cardiomyocytes. $\mathrm{J}$ Clin Invest. 2010; 120:1441-1453. [PubMed: 20364086]

574. Saez JC, Berthoud VM, Branes MC, Martinez AD, Beyer EC. Plasma membrane channels formed by connexins: Their regulation and functions. Physiol Rev. 2003; 83:1359-1400. [PubMed: 14506308]

575. Saez JC, Connor JA, Spray DC, Bennett MV. Hepatocyte gap junctions are permeable to the second messenger, inositol 1,4,5-trisphosphate, and to calcium ions. Proc Natl Acad Sci U S A. 1989; 86:2708-2712. [PubMed: 2784857]

576. Saez JC, Retamal MA, Basilio D, Bukauskas FF, Bennett MV. Connexin-based gap junction hemichannels: Gating mechanisms. Biochim Biophys Acta. 2005; 1711:215-224. [PubMed: 15955306]

577. Saez JC, Schalper KA, Retamal MA, Orellana JA, Shoji KF, Bennett MV. Cell membrane permeabilization via connexin hemichannels in living and dying cells. Exp Cell Res. 2010; 316:2377-2389. [PubMed: 20595004]

578. Saez JC, Spray DC, Nairn AC, Hertzberg E, Greengard P, Bennett MV. cAMP increases junctional conductance and stimulates phosphorylation of the $27-\mathrm{kDa}$ principal gap junction polypeptide. Proc Natl Acad Sci. 1986; 83:2473-2477. [PubMed: 3010311]

579. Sáez JC, Nairn AC, Czernik AJ, Fishman GI, Spray DC, Hertzberg EL. Phosphorylation of Connexin43 and the regulation of neonatal rat cardiac myocyte gap junctions. J Mol Cell Cardiol. 1997; 29:2131-2145. [PubMed: 9281445]

580. Saez JC, Nairn AC, Czernik AJ, Spray DC, Hertzberg EL, Greengard P, Bennett MVL. Phosphorylation of connexin 32, a hepatocyte gap-junction protein, by cAMP-dependent protein kinase, protein kinase $\mathrm{C}$ and Ca2+/calmodulin-dependent protein kinase II. Eur J Biochem. 1990; 192:263-273. [PubMed: 2170122]

581. Saffitz JE, Davis LM, Darrow BJ, Kanter HL, Laing JG, Beyer EC. The molecular basis of anisotropy: Role of gap junctions. J Cardiovasc Electrophysiol. 1995; 6:498-510. [PubMed: 7551319]

582. Sagar GD, Larson DM. Carbenoxolone inhibits junctional transfer and upregulates Connexin43 expression by a protein kinase A-dependent pathway. J Cell Biochem. 2006; 98:1543-1551. [PubMed: 16552723]

583. Saltman AE, Aksehirli TO, Valiunas V, Gaudette GR, Matsuyama N, Brink P, Krukenkamp IB. Gap junction uncoupling protects the heart against ischemia. J Thorac Cardiovasc Surg. 2002; 124:371-376. [PubMed: 12167798]

584. Sanchez HA, Orellana JA, Verselis VK, Saez JC. Metabolic inhibition increases activity of connexin-32 hemichannels permeable to Ca2+ in transfected HeLa cells. Am J Physiol Cell Physiol. 2009; 297:C665-C678. [PubMed: 19587218]

585. Sanders KM. A case for interstitial cells of Cajal as pacemakers and mediators of neurotransmission in the gastrointestinal tract. Gastroenterology. 1996; 111:492-515. [PubMed: 8690216] 
586. Sanders KM, Ordog T, Ward SM. Physiology and pathophysiology of the interstitial cells of Cajal: From bench to bedside. IV. Genetic and animal models of GI motility disorders caused by loss of interstitial cells of Cajal. Am J Physiol Gastrointest Liver Physiol. 2002; 282:G747G756. [PubMed: 11960771]

587. Sanderson MJ, Charles AC, Dirksen ER. Mechanical stimulation and intercellular communication increases intracellular Ca2+ in epithelial cells. Cell Regul. 1990; 1:585-596. [PubMed: 2078569]

588. Sandow SL, Haddock RE, Hill CE, Chadha PS, Kerr PM, Welsh DG, Plane F. What's where and why at a vascular myoendothelial microdomain signalling complex. Clin Exp Pharmacol Physiol. 2009; 36:67-76. [PubMed: 19018806]

589. Sanna E, Mascia MP, Klein RL, Whiting PJ, Biggio G, Harris RA. Actions of the general anesthetic propofol on recombinant human GABAA receptors: Influence of receptor subunits. J Pharmacol Exp Ther. 1995; 274:353-360. [PubMed: 7616420]

590. Sarieddine MZ, Scheckenbach KE, Foglia B, Maass K, Garcia I, Kwak BR, Chanson M. Connexin43 modulates neutrophil recruitment to the lung. J Cell Mol Med. 2009; 13:4560-4570. [PubMed: 19166484]

591. Sarma JD, Das S, Koval M. Regulation of connexin43 oligomerization is saturable. Cell Commun Adhes. 2005; 12:237-247. [PubMed: 16531319]

592. Sasseville' M, Gagnon MC, Guillemette C, Sullivan R, Gilchrist RB, Richard FJ. Regulation of gap junctions in porcine cumulus-oocyte complexes: Contributions of granulosa cell contact, gonadotropins, and lipid rafts. Mol Endocrinol. 2009; 23:700-710. [PubMed: 19228792]

593. Scemes E, Giaume C. Astrocyte calcium waves: What they are and what they do. Glia. 2006; 54:716-725. [PubMed: 17006900]

594. Schalper KA, Orellana JA, Berthoud VM, Saez JC. Dysfunctions of the diffusional membrane pathways mediated by hemichannels in inherited and acquired human diseases. Curr Vasc Pharmacol. 2009; 7:486-505. [PubMed: 19485891]

595. Schalper KA, Palacios-Prado N, Retamal MA, Shoji KF, Martinez AD, Saez JC. Connexin hemichannel composition determines the FGF-1-induced membrane permeability and free [Ca2+]i responses. Mol Biol Cell. 2008; 19:3501-3513. [PubMed: 18495870]

596. Scheckenbach KE, Crespin S, Kwak BR, Chanson M. Connexin channel-dependent signaling pathways in inflammation. J Vasc Res. 2011; 48:91-103. [PubMed: 20926890]

597. Schock SC, Leblanc D, Hakim AM, Thompson CS. ATP release by way of connexin 36 hemichannels mediates ischemic tolerance in vitro. Biochem Biophys Res Commun. 2008; 368:138-144. [PubMed: 18211823]

598. Schrickel JW, Kreuzberg MM, Ghanem A, Kim JS, Linhart M, Andrie R, Tiemann K, Nickenig G, Lewalter T, Willecke K. Normal impulse propagation in the atrioventricular conduction system of Cx30.2/Cx40 double deficient mice. J Mol Cell Cardiol. 2009; 46:644-652. [PubMed: 19248787]

599. Schubert AL, Schubert W, Spray DC, Lisanti MP. Connexin family members target to lipid raft domains and interact with caveolin-1. Biochemistry. 2002; 41:5754-5764. [PubMed: 11980479]

600. Schuetze SM, Goodenough DA. Dye transfer between cells of the embryonic chick lens becomes less sensitive to CO2 treatment with development. J Cell Biol. 1982; 92:694-705. [PubMed: 6806303]

601. Schultz BD, Singh AK, Devor DC, Bridges RJ. Pharmacology of CFTR chloride channel activity. Physiol Rev. 1999; 79:S109-S144. [PubMed: 9922378]

602. Schulz R, Boengler K, Totzeck A, Luo Y, Garcia-Dorado D, Heusch G. Connexin 43 in ischemic pre- and postconditioning. Heart Fail Rev. 2007; 12:261-266. [PubMed: 17516165]

603. Schwanke U, Konietzka I, Duschin A, Li X, Schulz R, Heusch G. No ischemic preconditioning in heterozygous connexin43-deficient mice. Am J Physiol Heart Circ Physiol. 2002; 283:H1740H1742. [PubMed: 12234831]

604. Segal SS. Regulation of blood flow in the microcirculation. Microcirculation. 2005; 12:33-45. [PubMed: 15804972]

605. Segal SS, Duling BR. Propagation of vasodilation in resistance vessels of the hamster: Development and review of a working hypothesis. Circ Res. 1987; 61:II20-II25. [PubMed: 3664984] 
606. Seki A, Duffy HS, Coombs W, Spray DC, Taffet SM, Delmar M. Modifications in the biophysical properties of connexin 43 channels by a peptide of the cytoplasmic loop region. Circ Res. 2004; 95:e22-e28. [PubMed: 15284189]

607. Sellitto C, Li L, White TW. Connexin50 is essential for normal postnatal lens cell proliferation. Invest Ophthalmol Vis Sci. 2004; 45:3196-3202. [PubMed: 15326140]

608. Serre-Beinier V, Bosco D, Zulianello L, Charollais A, Caille D, Charpantier E, Gauthier BR, Diaferia GR, Giepmans BN, Lupi R, Marchetti P, Deng S, Buhler L, Berney T, Cirulli V, Meda P. Cx36 makes channels coupling human pancreatic beta-cells, and correlates with insulin expression. Hum Mol Genet. 2009; 18:428-439. [PubMed: 19000992]

609. Serre-Beinier V, Le GS, Belluardo N, Trovato-Salinaro A, Charollais A, Haefliger JA, Condorelli DF, Meda P. Cx36 preferentially connects beta-cells within pancreatic islets. Diabetes. 2000; 49:727-734. [PubMed: 10905480]

610. Severs NJ. The cardiac gap junction and intercalated disc. Int J Cardiol. 1990; 26:137-173. [PubMed: 2406208]

611. Severs NJ. Gap junction remodeling and cardiac arrhythmogenesis: Cause or coincidence? J Cell Mol Med. 2001; 5:355-366. [PubMed: 12067469]

612. Severs NJ. Gap junction remodeling in heart failure. J Card Fail. 2002; 8:S293-S299. [PubMed: 12555135]

613. Severs NJ, Bruce AF, Dupont E, Rothery S. Remodelling of gap junctions and connexin expression in diseased myocardium. Cardiovasc Res. 2008; 80:9-19. [PubMed: 18519446]

614. Severs NJ, Coppen SR, Dupont E, Yeh HI, Ko YS, Matsushita T. Gap junction alterations in human cardiac disease. Cardiovasc Res. 2004; 62:368-377. [PubMed: 15094356]

615. Severs NJ, Dupont E, Coppen SR, Halliday D, Inett E, Baylis D, Rothery S. Remodelling of gap junctions and connexin expression in heart disease. Biochim Biophys Acta. 2004; 1662:138-148. [PubMed: 15033584]

616. Shah MM, Martinez AM, Fletcher WH. The connexin43 gap junction protein is phosphorylated by protein kinase A and protein kinase C: In vivo and in vitro studies. Mol Cell Biochem. 2002; 238:57-68. [PubMed: 12349910]

617. Shaw RM, Fay AJ, Puthenveedu MA, von ZM, Jan YN, Jan LY. Microtubule plus-end-tracking proteins target gap junctions directly from the cell interior to adherens junctions. Cell. 2007; 128:547-560. [PubMed: 17289573]

618. Shearer D, Ens W, Standing K, Valdimarsson G. Posttranslational modifications in lens fiber connexins identified by off-line-HPLC MALDIquadrupole time-of-flight mass spectrometry. Invest Ophthalmol Vis Sci. 2008; 49:1553-1562. [PubMed: 18385075]

619. Shi Y. Serine/threonine phosphatases: Mechanism through structure. Cell. 2009; 139:468-484. [PubMed: 19879837]

620. Shibayama J, Gutierrez C, Gonzalez D, Kieken F, Seki A, Carrion JR, Sorgen PL, Taffet SM, Barrio LC, Delmar M. Effect of charge substitutions at residue his-142 on voltage gating of connexin43 channels. Biophys J. 2006; 91:4054-4063. [PubMed: 16963503]

621. Shibayama J, Lewandowski R, Kieken F, Coombs W, Shah S, Sorgen PL, Taffet SM, Delmar M. Identification of a novel peptide that interferes with the chemical regulation of connexin43. Circ Res. 2006; 98:1365-1372. [PubMed: 16690883]

622. Shimizu K, Shimoichi Y, Hinotsume D, Itsuzaki Y, Fujii H, Honoki K, Tsujiuchi T. Reduced expression of the Connexin26 gene and its aberrant DNA methylation in rat lung adenocarcinomas induced by N-nitrosobis( 2-hydroxypropyl)amine. Mol Carcinog. 2006; 45:710-714. [PubMed: 16637067]

623. Shimono M, Young LC, Matsuzaki H, Ishikawa H, Inoue T, Hashimoto S, Muramatsu T. Connexins in salivary glands. Eur J Morphol. 2000; 38:257-261. [PubMed: 10980677]

624. Shintani-Ishida K, Uemura K, Yoshida K. Hemichannels in cardiomyocytes open transiently during ischemia and contribute to reperfusion injury following brief ischemia. Am J Physiol Heart Circ Physiol. 2007; 293:H1714-H1720. [PubMed: 17557925]

625. Shiosaka S, Yamamoto T, Hertzberg EL, Nagy JI. Gap junction protein in rat hippocampus: Correlative light and electron microscope immunohistochemical localization. J Comp Neurol. 1989; 281:282-297. [PubMed: 2540227] 
626. Shiraishi M, Harris RA. Effects of alcohols and anesthetics on recombinant voltage-gated Na+ channels. J Pharmacol Exp Ther. 2004; 309:987-994. [PubMed: 14978193]

627. Shiroshita-Takeshita A, Sakabe M, Haugan K, Hennan JK, Nattel S. Model-dependent effects of the gap junction conduction-enhancing antiarrhythmic peptide rotigaptide (ZP123) on experimental atrial fibrillation in dogs. Circulation. 2007; 115:310-318. [PubMed: 17224477]

628. Shynlova OP, Oldenhof AD, Liu M, Langille L, Lye SJ. Regulation of c-fos expression by static stretch in rat myometrial smooth muscle cells. Am J Obstet Gynecol. 2002; 186:1358-1365. [PubMed: 12066122]

629. Siller-Jackson AJ, Burra S, Gu S, Xia X, Bonewald LF, Sprague E, Jiang JX. Adaptation of connexin 43-hemichannel prostaglandin release to mechanical loading. J Biol Chem. 2008; 283:26374-26382. [PubMed: 18676366]

630. Simon AM, Goodenough DA. Diverse functions of vertebrate gap junctions. Trends Cell Biol. 1998; 8:477-483. [PubMed: 9861669]

631. Simon AM, Goodenough DA, Li E, Paul DL. Female infertility in mice lacking connexin 37. Nature. 1997; 385:525-529. [PubMed: 9020357]

632. Simon AM, Goodenough DA, Paul DL. Mice lacking connexin40 have cardiac conduction abnormalities characteristic of atrioventricular block and bundle branch block. Curr Biol. 1998; 8:295-298. [PubMed: 9501069]

633. Sims SM, Daniel EE, Garfield RE. Improved electrical coupling in uterine smooth muscle is associated with increased numbers of gap junctions at parturition. J Gen Physiol. 1982; 80:353375. [PubMed: 7142952]

634. Singal R, Tu ZJ, Vanwert JM, Ginder GD, Kiang DT. Modulation of the connexin26 tumor suppressor gene expression through methylation in human mammary epithelial cell lines. Anticancer Res. 2000; 20:59-64. [PubMed: 10769635]

635. Singh MV, Bhatnagar R, Price CJ, Malhotra SK. Gap junctions in 9L and C6 glioma cells: Correlation with growth characteristics. Cytobios. 1997; 89:209-225. [PubMed: 9418017]

636. Sipos A, Vargas SL, Toma I, Hanner F, Willecke K, Peti-Peterdi J. Connexin 30 deficiency impairs renal tubular ATP release and pressure natriuresis. J Am Soc Nephrol. 2009; 20:17241732. [PubMed: 19478095]

637. Sirnes S, Honne H, Ahmed D, Danielsen SA, Rognum TO, Meling GI, Leithe E, Rivedal E, Lothe RA, Lind GE. DNA methylation analyses of the connexin gene family reveal silencing of GJC1 (Connexin45) by promoter hypermethylation in colorectal cancer. Epigenetics. 2011; 6:602-609. [PubMed: 21406965]

638. Sirnes S, Kjenseth A, Leithe E, Rivedal E. Interplay between PKC and the MAP kinase pathway in Connexin43 phosphorylation and inhibition of gap junction intercellular communication. Biochem Biophys Res Commun. 2009; 382:41-45. [PubMed: 19258009]

639. Smyth JW, Hong TT, Gao D, Vogan JM, Jensen BC, Fong TS, Simpson PC, Stainier DY, Chi NC, Shaw RM. Limited forward trafficking of connexin 43 reduces cell-cell coupling in stressed human and mouse myocardium. J Clin Invest. 2010; 120:266-279. [PubMed: 20038810]

640. Soder BL, Propst JT, Brooks TM, Goodwin RL, Friedman HI, Yost MJ, Gourdie RG. The connexin43 carboxyl-terminal peptide ACT1 modulates the biological response to silicone implants. Plast Reconstr Surg. 2009; 123:1440-1451. [PubMed: 19407614]

641. Sohl G, Maxeiner S, Willecke K. Expression and functions of neuronal gap junctions. Nat Rev Neurosci. 2005; 6:191-200. [PubMed: 15738956]

642. Sohl G, Willecke K. An update on connexin genes and their nomenclature in mouse and man. Cell Commun Adhes. 2003; 10:173-180. [PubMed: 14681012]

643. Solan J, Lampe P. Key Connexin 43 phosphorylation events regulate the gap junction life cycle. J Membr Biol. 2007; 217:35-41. [PubMed: 17629739]

644. Solan JL, Fry MD, TenBroek EM, Lampe PD. Connexin43 phosphorylation at S368 is acute during $\mathrm{S}$ and G2/M and in response to protein kinase C activation. J Cell Sci. 2003; 116:22032211. [PubMed: 12697837]

645. Solan JL, Lampe PD. Connexin43 in LA-25 cells with active v-src is phosphorylated on Y247, Y265, S262, S279/282, and S368 via multiple signaling pathways. Cell Commun Adhes. 2008; 15:75-84. [PubMed: 18649180] 
646. Solan JL, Marquez-Rosado L, Sorgen PL, Thornton PJ, Gafken PR, Lampe PD. Phosphorylation at $\mathrm{S} 365$ is a gatekeeper event that changes the structure of $\mathrm{Cx} 43$ and prevents down-regulation by PKC. J Cell Biol. 2007; 179:1301-1309. [PubMed: 18086922]

647. Sorgen PL, Duffy HS, Cahill SM, Coombs W, Spray DC, Delmar M, Girvin ME. Sequencespecific resonance assignment of the carboxyl terminal domain of Connexin43. J Biomol NMR. 2002; 23:245-246. [PubMed: 12238598]

648. Sorgen PL, Duffy HS, Sahoo P, Coombs W, Delmar M, Spray DC. Structural changes in the carboxyl terminus of the gap junction protein connexin43 indicates signaling between binding domains for c-Src and zonula occludens-1. J Biol Chem. 2004; 279:54695-54701. [PubMed: 15492000]

649. Sorgen PL, Duffy HS, Spray DC, Delmar M. pH-dependent dimerization of the carboxyl terminal domain of Cx43. Biophys J. 2004; 87:574-581. [PubMed: 15240490]

650. Spray DC, Iacobas DA. Organizational principles of the connexin-related brain transcriptome. J Membr Biol. 2007; 218:39-47. [PubMed: 17657523]

651. Sridharan S, Brehm R, Bergmann M, Cooke PS. Role of connexin 43 in Sertoli cells of testis. Ann N Y Acad Sci. 2007; 1120:131-143. [PubMed: 17905936]

652. Sridharan S, Simon L, Meling DD, Cyr DG, Gutstein DE, Fishman GI, Guillou F, Cooke PS. Proliferation of adult sertoli cells following conditional knockout of the gap junctional protein GJA1 (connexin 43) in mice. Biol Reprod. 2007; 76:804-812. [PubMed: 17229929]

653. Srinivas M, Calderon DP, Kronengold J, Verselis VK. Regulation of connexin hemichannels by monovalent cations. J Gen Physiol. 2006; 127:67-75. [PubMed: 16380444]

654. Srinivas M, Hopperstad MG, Spray DC. Quinine blocks specific gap junction channel subtypes. Proc Natl Acad Sci U S A. 2001; 98:10942-10947. [PubMed: 11535816]

655. Srinivas M, Spray DC. Closure of gap junction channels by arylaminobenzoates. Mol Pharmacol. 2003; 63:1389-1397. [PubMed: 12761350]

656. Srinivas M, Costa M, Gao Y, Fort A, Fishman GI, Spray DC. Voltage dependence of macroscopic and unitary currents of gap junction channels formed by mouse connexin50 expressed in rat neuroblastoma cells. J Physiol. 1999; 517:673-689. [PubMed: 10358109]

657. Srisakuldee W, Jeyaraman MM, Nickel BE, Tanguy Sp, Jiang ZS, Kardami E. Phosphorylation of connexin-43 at serine 262 promotes a cardiac injury-resistant state. Cardiovasc Res. 2009; 83:672-681. [PubMed: 19423616]

658. St-Pierre N, Dufresne J, Rooney AA, Cyr DG. Neonatal hypothyroidism alters the localization of gap junctional protein connexin 43 in the testis and messenger RNA levels in the epididymis of the rat. Biol Reprod. 2003; 68:1232-1240. [PubMed: 12606457]

659. Stahlhut M, Petersen JS, Hennan JK, Ramirez MT. The antiarrhythmic peptide rotigaptide (ZP123) increases connexin 43 protein expression in neonatal rat ventricular cardiomyocytes. Cell Commun Adhes. 2006; 13:21-27. [PubMed: 16613777]

660. Stauffer PL, Zhao H, Luby-Phelps K, Moss RL, Star RA, Muallem S. Gap junction communication modulates [Ca2+]i oscillations and enzyme secretion in pancreatic acini. J Biol Chem. 1993; 268:19769-19775. [PubMed: 8366115]

661. Steele J, Lyon MF, Favor J, Guillot PV, Boyd Y, Church RL. A mutation in the connexin 50 (Cx50) gene is a candidate for the No2 mouse cataract. Curr Eye Res. 1998; 17:883-889. [PubMed: 9746435]

662. Stergiopoulos K, Alvarado JL, Mastroianni M, Ek-Vitorin JF, Taffet SM, Delmar M. Heterodomain interactions as a mechanism for the regulation of connexin channels. Circ Res. 1999; 84:1144-1155. [PubMed: 10347089]

663. Stout CE, Costantin JL, Naus CC, Charles AC. Intercellular calcium signaling in astrocytes via ATP release through connexin hemichannels. J Biol Chem. 2002; 277:10482-10488. [PubMed: 11790776]

664. Straub AC, Billaud M, Johnstone SR, Best AK, Yemen S, Dwyer ST, Looft-Wilson R, Lysiak JJ, Gaston B, Palmer L, Isakson BE. Compartmentalized Connexin 43 S-nitrosylation/ denitrosylation regulates heterocellular communication in the vessel wall. Arterioscler Thromb Vasc Biol. 2011; 31:399-407. [PubMed: 21071693] 
665. Stumpel F, Ott T, Willecke K, Jungermann K. Connexin 32 gap junctions enhance stimulation of glucose output by glucagon and noradrenaline in mouse liver. Hepatology. 1998; 28:1616-1620. [PubMed: 9828226]

666. Su V, Nakagawa R, Koval M, Lau AF. Ubiquitin-independent proteasomal degradation of endoplasmic reticulum-localized connexin43 mediated by CIP75. J Biol Chem. 2010; 285:40979-40990. [PubMed: 20940304]

667. Suchyna TM, Nitsche JM, Chilton M, Harris AL, Veenstra RD, Nicholson BJ. Different ionic selectivities for connexins 26 and 32 produce rectifying gap junction channels. Biophys J. 1999; 77:2968-2987. [PubMed: 10585920]

668. Sun Z, Zhang DQ, McMahon DG. Zinc modulation of hemi-gap-junction channel currents in retinal horizontal cells. J Neurophysiol. 2009; 101:1774-1780. [PubMed: 19176613]

669. Takeda A, Hashimoto E, Yamamura H, Shimazu T. Phosphorylation of liver gap junction protein by protein kinase C. FEBS Letters. 1987; 210:169-172. [PubMed: 3025025]

670. Takens-Kwak BR, Jongsma HJ, Rook MB, Van Ginneken AC. Mechanism of heptanol-induced uncoupling of cardiac gap junctions: A perforated patch-clamp study. Am J Physiol. 1992; 262:C1531-C1538. [PubMed: 1616013]

671. Takeuchi H, Jin S, Wang J, Zhang G, Kawanokuchi J, Kuno R, Sonobe Y, Mizuno T, Suzumura A. Tumor necrosis factor-alpha induces neurotoxicity via glutamate release from hemichannels of activated microglia in an autocrine manner. J Biol Chem. 2006; 281:21362-21368. [PubMed: 16720574]

672. Tan LW, Bianco T, Dobrovic A. Variable promoter region CpG island methylation of the putative tumor suppressor gene Connexin 26 in breast cancer. Carcinogenesis. 2002; 23:231-236. [PubMed: 11872627]

673. TenBroek EM, Lampe PD, Solan JL, Reynhout JK, Johnson RG. Ser364 of connexin43 and the upregulation of gap junction assembly by cAMP. J Cell Biol. 2001; 155:1307-1318. [PubMed: 11756479]

674. Teubner B, Degen J, Sohl G, Guldenagel M, Bukauskas FF, Trexler EB, Verselis VK, De Zeeuw CI, Lee CG, Kozak CA, Petrasch-Parwez E, Dermietzel R, Willecke K. Functional expression of the murine connexin 36 gene coding for a neuron-specific gap junctional protein. J Membr Biol. 2000; 176:249-262. [PubMed: 10931976]

675. Teubner B, Odermatt B, Guldenagel M, Sohl G, Degen J, Bukauskas F, Kronengold J, Verselis VK, Jung YT, Kozak CA, Schilling K, Willecke K. Functional expression of the new gap junction gene connexin47 transcribed in mouse brain and spinal cord neurons. J Neurosci. 2001; 21:1117-1126. [PubMed: 11160382]

676. Teunissen BE, Jansen AT, van Amersfoorth SC, O’Brien TX, Jongsma HJ, Bierhuizen MF. Analysis of the rat connexin 43 proximal promoter in neonatal cardiomyocytes. Gene. 2003; 322:123-136. [PubMed: 14644504]

677. Thimm J, Mechler A, Lin H, Rhee S, Lal R. Calcium-dependent open/closed conformations and interfacial energy maps of reconstituted hemichannels. J Biol Chem. 2005; 280:10646-10654. [PubMed: 15615707]

678. Thomas MA, Zosso N, Scerri I, Demaurex N, Chanson M, Staub O. A tyrosine-based sorting signal is involved in connexin43 stability and gap junction turnover. J Cell Sci. 2003; 116:22132222. [PubMed: 12730291]

679. Thomas SA, Schuessler RB, Berul CI, Beardslee MA, Beyer EC, Mendelsohn ME, Saffitz JE. Disparate effects of deficient expression of connexin43 on atrial and ventricular conduction: Evidence for chamber-specific molecular determinants of conduction. Circulation. 1998; 97:686691. [PubMed: 9495305]

680. Thomas T, Jordan K, Laird DW. Role of cytoskeletal elements in the recruitment of Cx43-GFP and Cx26-YFP into gap junctions. Cell Commun Adhes. 2001; 8:231-236. [PubMed: 12064594]

681. Thomas T, Jordan K, Simek J, Shao Q, Jedeszko C, Walton P, Laird DW. Mechanisms of Cx43 and Cx26 transport to the plasma membrane and gap junction regeneration. J Cell Sci. 2005; 118:4451-4462. [PubMed: 16159960]

682. Thompson RJ, Zhou N, MacVicar BA. Ischemia opens neuronal gap junction hemichannels. Science. 2006; 312:924-927. [PubMed: 16690868] 
683. Tibbitts TT, Caspar DL, Phillips WC, Goodenough DA. Diffraction diagnosis of protein folding in gap junction connexons. Biophys J. 1990; 57:1025-1036. [PubMed: 2160297]

684. Tong D, Lu X, Wang HX, Plante I, Lui E, Laird DW, Bai D, Kidder GM. A dominant loss-offunction GJA1 (Cx43) mutant impairs parturition in the mouse. Biol Reprod. 2009; 80:10991106. [PubMed: 19176884]

685. Török K, Stauffer K, Evans WH. Connexin 32 of gap junctions contains two cytoplasmic calmodulin-binding domains. Biochem J. 1997; 326:479-483. [PubMed: 9291121]

686. Totzeck A, Boengler K, van de Sand A, Konietzka I, Gres P, Garcia-Dorado D, Heusch G, Schulz R. No impact of protein phosphatases on connexin 43 phosphorylation in ischemic preconditioning. Am J Physiol Heart Circ Physiol. 2008; 295:H2106-H2112. [PubMed: 18835920]

687. Toyofuku T, Akamatsu Y, Zhang H, Kuzuya T, Tada M, Hori M. c-Src Regulates the Interaction between Connexin-43 and ZO-1 in cardiac myocytes. J Biol Chem. 2001; 276:1780-1788. [PubMed: 11035005]

688. Tran Van NG, Clair C, Bruzzone R, Mesnil M, Sansonetti P, Combettes L. Connexin-dependent inter-cellular communication increases invasion and dissemination of Shigella in epithelial cells. Nat Cell Biol. 2003; 5:720-726. [PubMed: 12844145]

689. Traub O, Eckert R, LichtenbergFrate H, Elfgang C, Bastide B, Scheidtmann KH, Hulser DF, Willecke K. Immunochemical and electrophysiological characterization of murine Connexin40 and Connexin43 in mouse-tissues and transfected human-cells. Eur J Cell Biol. 1994; 64:101112. [PubMed: 7957300]

690. Traub O, Look J, Dermietzel R, Brummer F, Hulser D, Willecke K. Comparative characterization of the 21-kD and 26-kD gap junction proteins in murine liver and cultured hepatocytes. J Cell Biol. 1989; 108:1039-1051. [PubMed: 2537831]

691. Traub O, Look J, Paul D, Willecke K. Cyclic adenosine monophosphate stimulates biosynthesis and phosphorylation of the $26 \mathrm{kDa}$ gap junction protein in cultured mouse hepatocytes. Eur J Cell Biol. 1987; 43:48-54. [PubMed: 3032632]

692. Traub RD, Pais I, Bibbig A, Lebeau FE, Buhl EH, Hormuzdi SG, Monyer H, Whittington MA. Contrasting roles of axonal (pyramidal cell) and dendritic (interneuron) electrical coupling in the generation of neuronal network oscillations. Proc Natl Acad Sci U S A. 2003; 100:1370-1374. [PubMed: 12525690]

693. Trexler EB, Bukauskas FF, Bennett MV, Bargiello TA, Verselis VK. Rapid and direct effects of $\mathrm{pH}$ on connexins revealed by the connexin46 hemichannel preparation. J Gen Physiol. 1999; 113:721-742. [PubMed: 10228184]

694. Trexler EB, Bukauskas FF, Kronengold J, Bargiello TA, Verselis VK. The first extracellular loop domain is a major determinant of charge selectivity in connexin46 channels. Biophys J. 2000; 79:3036-3051. [PubMed: 11106610]

695. Tsujiuchi T, Shimizu K, Itsuzaki Y, Onishi M, Sugata E, Fujii H, Honoki K. CpG site hypermethylation of E-cadherin and Connexin26 genes in hepatocellular carcinomas induced by a choline-deficient L-Amino Acid-defined diet in rats. Mol Carcinog. 2007; 46:269-274. [PubMed: 17295234]

696. Tyagi N, Vacek JC, Givvimani S, Sen U, Tyagi SC. Cardiac specific deletion of N-methyl-daspartate receptor 1 ameliorates mtMMP-9 mediated autophagy/mitophagy in hyperhomocysteinemia. J Recept Signal Transduct Res. 2010; 30:78-87. [PubMed: 20170426]

697. Uhlenberg B, Schuelke M, Ruschendorf F, Ruf N, Kaindl AM, Henneke M, Thiele H, Stoltenburg-Didinger G, Aksu F, Topaloglu H, Nurnberg P, Hubner C, Weschke B, Gartner J. Mutations in the gene encoding gap junction protein alpha 12 (connexin 46.6) cause PelizaeusMerzbacher-like disease. Am J Hum Genet. 2004; 75:251-260. [PubMed: 15192806]

698. Unger VM, Kumar NM, Gilula NB, Yeager M. Three-dimensional structure of a recombinant gap junction membrane channel. Science. 1999; 283:1176-1180. [PubMed: 10024245]

699. Unwin PN, Zampighi G. Structure of the junction between communicating cells. Nature. 1980; 283:545-549. [PubMed: 7354837]

700. Urschel S, H+Âher T, Schubert T, Alev C, S+Âhl G, W+Ârsd+Ârfer P, Asahara T, Dermietzel R, Weiler R, Willecke K. Protein kinase A-mediated phosphorylation of Connexin36 in mouse 
retina results in decreased gap junctional communication between AII amacrine cells. J Biol Chem. 2006; 281:33163-33171. [PubMed: 16956882]

701. Valiunas V. Biophysical properties of connexin-45 gap junction hemichannels studied in vertebrate cells. J Gen Physiol. 2002; 119:147-164. [PubMed: 11815665]

702. Valiunas V, Gemel J, Brink PR, Beyer EC. Gap junction channels formed by coexpressed connexin40 and connexin43. Am J Physiol Heart Circ Physiol. 2001; 281:H1675-H1689. [PubMed: 11557558]

703. Valiunas V, Mui R, McLachlan E, Valdimarsson G, Brink PR, White TW. Biophysical characterization of zebrafish connexin35 hemichannels. Am J Physiol Cell Physiol. 2004; 287:C1596-C1604. [PubMed: 15282192]

704. Valiunas V, Polosina YY, Miller H, Potapova IA, Valiuniene L, Doronin S, Mathias RT, Robinson RB, Rosen MR, Cohen IS, Brink PR. Connexin-specific cell-to-cell transfer of short interfering RNA by gap junctions. J Physiol. 2005; 568:459-468. [PubMed: 16037090]

705. Valiunas V, Weingart R. Electrical properties of gap junction hemichannels identified in transfected HeLa cells. Pflugers Arch. 2000; 440:366-379. [PubMed: 10954323]

706. van der Velden HM, van Kempen MJ, Wijffels MC, van ZM, Groenewegen WA, Allessie MA, Jongsma HJ. Altered pattern of connexin40 distribution in persistent atrial fibrillation in the goat. J Cardiovasc Electrophysiol. 1998; 9:596-607. [PubMed: 9654224]

707. van Kempen MJ, Fromaget C, Gros D, Moorman AF, Lamers WH. Spatial distribution of connexin 43, the major cardiac gap junction protein, in the developing and adult rat heart. Circ Res. 1991; 68:1638-1651. [PubMed: 1645233]

708. van Rijen HV, Eckardt D, Degen J, Theis M, Ott T, Willecke K, Jongsma HJ, Opthof T, de Bakker JM. Slow conduction and enhanced anisotropy increase the propensity for ventricular tachyarrhythmias in adult mice with induced deletion of connexin43. Circulation. 2004; 109:1048-1055. [PubMed: 14967725]

709. van Rijen HV, van Veen TA, Gros D, Wilders R, de Bakker JM. Connexins and cardiac arrhythmias. Adv Cardiol. 2006; 42:150-160. [PubMed: 16646589]

710. van Rijen HVM, van Veen TAB, Hermans MMP, Jongsma HJ. Human connexin40 gap junction channels are modulated by cAMP. Cardiovasc Res. 2000; 45:941-951. [PubMed: 10728420]

711. van Veen TAB, van Rijen HVM, Jongsma HJ. Electrical conductance of mouse connexin45 gap junction channels is modulated by phosphorylation. Cardiovasc Res. 2000; 46:496-510. [PubMed: 10912460]

712. van Zeijl L, Ponsioen B, Giepmans BN, Ariaens A, Postma FR, Varnai P, Balla T, Divecha N, Jalink K, Moolenaar WH. Regulation of connexin43 gap junctional communication by phosphatidylinositol 4,5-bisphosphate. J Cell Biol. 2007; 177:881-891. [PubMed: 17535964]

713. VanSlyke JK, Musil LS. Dislocation and degradation from the ER are regulated by cytosolic stress. J Cell Biol. 2002; 157:381-394. [PubMed: 11980915]

714. VanSlyke JK, Naus CC, Musil LS. Conformational maturation and post-ER multisubunit assembly of gap junction proteins. Mol Biol Cell. 2009; 20:2451-2463. [PubMed: 19297523]

715. Varanda WA, de Carvalho AC. Intercellular communication between mouse Leydig cells. Am J Physiol. 1994; 267:C563-C569. [PubMed: 8074190]

716. Veenstra RD, Wang HZ, Beblo DA, Chilton MG, Harris AL, Beyer EC, Brink PR. Selectivity of connexin-specific gap junctions does not correlate with channel conductance. Circ Res. 1995; 77:1156-1165. [PubMed: 7586229]

717. Veenstra RD, Wang HZ, Beyer EC, Brink PR. Selective dye and ionic permeability of gap junction channels formed by connexin45. Circ Res. 1994; 75:483-490. [PubMed: 7520372]

718. Veenstra RD, Wang HZ, Beyer EC, Ramanan SV, Brink PR. Connexin37 forms high conductance gap junction channels with subconductance state activity and selective dye and ionic permeabilities. Biophys J. 1994; 66:1915-1928. [PubMed: 7521227]

719. Veitch GI, Gittens JE, Shao Q, Laird DW, Kidder GM. Selective assembly of connexin37 into heterocellular gap junctions at the oocyte/granulosa cell interface. J Cell Sci. 2004; 117:26992707. [PubMed: 15138288] 
720. Velasquez Almonacid LA, Tafuri S, Dipineto L, Matteoli G, Fiorillo E, Della MR, Fioretti A, Menna LF, Staiano N. Role of connexin-43 hemichannels in the pathogenesis of Yersinia enterocolitica. Vet J. 2009; 182:452-457. [PubMed: 18824377]

721. Vergara L, Bao X, Cooper M, Bello-Reuss E, Reuss L. Gapjunctional hemichannels are activated by ATP depletion in human renal proximal tubule cells. J Membr Biol. 2003; 196:173-184. [PubMed: 14724743]

722. Verheule S, van Kempen MJ, te Welscher PH, Kwak BR, Jongsma HJ. Characterization of gap junction channels in adult rabbit atrial and ventricular myocardium. Circ Res. 1997; 80:673-681. [PubMed: 9130448]

723. Verma V, Larsen BD, Coombs W, Lin X, Sarrou E, Taffet SM, Delmar M. Design and characterization of the first peptidomimetic molecule that prevents acidification-induced closure of cardiac gap junctions. Heart Rhythm. 2010; 7:1491-1498. [PubMed: 20601149]

724. Verma V, Larsen BD, Coombs W, Lin X, Spagnol G, Sorgen PL, Taffet SM, Delmar M. Novel pharmacophores of connexin43 based on the "RXP" series of Cx43-binding peptides. Circ Res. 2009; 105:176-184. [PubMed: 19556520]

725. Verselis VK, Ginter CS, Bargiello TA. Opposite voltage gating polarities of two closely related connexins. Nature. 1994; 368:348-351. [PubMed: 8127371]

726. Verselis VK, Srinivas M. Divalent cations regulate connexin hemichannels by modulating intrinsic voltage-dependent gating. J Gen Physiol. 2008; 132:315-327. [PubMed: 18695008]

727. Verselis VK, Trexler EB, Bukauskas FF. Connexin hemichannels and cell-cell channels: Comparison of properties. Braz J Med Biol Res. 2000; 33:379-389. [PubMed: 10775302]

728. Vinken M, De Rop E, Decrock E, De Vuyst E, Leybaert L, Vanhaecke T, Rogiers V. Epigenetic regulation of gap junctional intercellular communication: More than a way to keep cells quiet? Biochim Biophys Acta. 2009; 1795:53-61. [PubMed: 18801412]

729. Vinken M, Henkens T, Vanhaecke T, Papeleu P, Geerts A, Van Rossen E, Chipman JK, Meda P, Rogiers V. Trichostatin A enhances gap junctional intercellular communication in primary cultures of adult rat hepatocytes. Toxicol Sci. 2006; 91:484-492. [PubMed: 16531468]

730. Wagner C, de WC, Kurtz L, Grunberger C, Kurtz A, Schweda F. Connexin40 is essential for the pressure control of renin synthesis and secretion. Circ Res. 2007; 100:556-563. [PubMed: 17255527]

731. Wagner C, Jobs A, Schweda F, Kurtz L, Kurt B, Lopez ML, Gomez RA, van Veen TA, de WC, Kurtz A. Selective deletion of Connexin 40 in renin-producing cells impairs renal baroreceptor function and is associated with arterial hypertension. Kidney Int. 2010; 78:762-768. [PubMed: 20686449]

732. Wang HX, Tong D, El-Gehani F, Tekpetey FR, Kidder GM. Connexin expression and gap junctional coupling in human cumulus cells: Contribution to embryo quality. J Cell Mol Med. 2009; 13:972-984. [PubMed: 18505471]

733. Wang HZ, Veenstra RD. Monovalent ion selectivity sequences of the rat connexin43 gap junction channel. J Gen Physiol. 1997; 109:491-507. [PubMed: 9101407]

734. Wang J, Ma M, Locovei S, Keane RW, Dahl G. Modulation of membrane channel currents by gap junction protein mimetic peptides: Size matters. Am J Physiol Cell Physiol. 2007; 293:C1112-C1119. [PubMed: 17652431]

735. Wang X, Li L, Peracchia LL, Peracchia C. Chimeric evidence for a role of the connexin cytoplasmic loop in gap junction channel gating. Pflugers Arch. 1996; 431:844-852. [PubMed: 8927500]

736. Wang Z, Schey KL. Phosphorylation and truncation sites of bovine lens connexin 46 and connexin 50. Exp Eye Res. 2009; 89:898-904. [PubMed: 19646399]

737. Ward SM, Sanders KM. Physiology and pathophysiology of the interstitial cell of Cajal: From bench to bedside. I. Functional development and plasticity of interstitial cells of Cajal networks. Am J Physiol Gastrointest Liver Physiol. 2001; 281:G602-G611. [PubMed: 11518672]

738. Warn-Cramer BJ, Cottrell GT, Burt JM, Lau AF. Regulation of Connexin-43 gap junctional intercellular communication by mitogenactivated protein kinase. J Biol Chem. 1998; 273:91889196. [PubMed: 9535909] 
739. Warn-Cramer BJ, Lampe PD, Kurata WE, Kanemitsu MY, Loo LWM, Eckhart W, Lau AF. Characterization of the mitogen-activated protein kinase phosphorylation sites on the Connexin-43 gap junction protein. J Biol Chem. 1996; 271:3779-3786. [PubMed: 8631994]

740. Weinbrenner C, Baines CP, Liu GS, Armstrong SC, Ganote CE, Walsh AH, Honkanen RE, Cohen MV, Downey JM. Fostriecin, an inhibitor of protein phosphatase 2A, limits myocardial infarct size even when administered after onset of ischemia. Circulation. 1998; 98:899-905. [PubMed: 9738645]

741. Weissman TA, Riquelme PA, Ivic L, Flint AC, Kriegstein AR. Calcium waves propagate through radial glial cells and modulate proliferation in the developing neocortex. Neuron. 2004; 43:647661. [PubMed: 15339647]

742. Werner R, Levine E, Rabadan-Diehl C, Dahl G. Gating properties of connexin32 cell-cell channels and their mutants expressed in Xenopus oocytes. Proc Biol Sci. 1991; 243:5-11. [PubMed: 1673244]

743. White TW. Functional analysis of human Cx26 mutations associated with deafness. Brain Res Brain Res Rev. 2000; 32:181-183. [PubMed: 10751668]

744. White TW, Deans MR, O’Brien J, Al-Ubaidi MR, Goodenough DA, Ripps H, Bruzzone R. Functional characteristics of skate connexin35, a member of the gamma subfamily of connexins expressed in the vertebrate retina. Eur J Neurosci. 1999; 11:1883-1890. [PubMed: 10336656]

745. White TW, Goodenough DA, Paul DL. Targeted ablation of connexin50 in mice results in microphthalmia and zonular pulverulent cataracts. J Cell Biol. 1998; 143:815-825. [PubMed: 9813099]

746. White TW, Paul DL. Genetic diseases and gene knockouts reveal diverse connexin functions. Annu Rev Physiol. 1999; 61:283-310. [PubMed: 10099690]

747. White TW, Paul DL, Goodenough DA, Bruzzone R. Functional analysis of selective interactions among rodent connexins. Mol Biol Cell. 1995; 6:459-470. [PubMed: 7542941]

748. Winterhager E, Pielensticker N, Freyer J, Ghanem A, Schrickel JW, Kim JS, Behr R, Grummer R, Maass K, Urschel S, Lewalter T, Tiemann K, Simoni M, Willecke K. Replacement of connexin 43 by connexin 26 in transgenic mice leads to dysfunctional reproductive organs and slowed ventricular conduction in the heart. BMC Dev Biol. 2007; 726

749. Wolvetang EJ, Pera MF, Zuckerman KS. Gap junction mediated transport of shRNA between human embryonic stem cells. Biochem Biophys Res Commun. 2007; 363:610-615. [PubMed: 17900528]

750. Wong CW, Burger F, Pelli G, Mach F, Kwak BR. Dual benefit of reduced Cx43 on atherosclerosis in LDL receptor-deficient mice. Cell Commun Adhes. 2003; 10:395-400. [PubMed: 14681047]

751. Wong CW, Christen T, Pfenniger A, James RW, Kwak BR. Do allelic variants of the connexin37 1019 gene polymorphism differentially predict for coronary artery disease and myocardial infarction? Atherosclerosis. 2007; 191:355-361. [PubMed: 16677656]

752. Wong CW, Christen T, Roth I, Chadjichristos CE, Derouette JP, Foglia BF, Chanson M, Goodenough DA, Kwak BR. Connexin37 protects against atherosclerosis by regulating monocyte adhesion. Nat Med. 2006; 12:950-954. [PubMed: 16862155]

753. Wright CS, van Steensel MA, Hodgins MB, Martin PE. Connexin mimetic peptides improve cell migration rates of human epidermal keratinocytes and dermal fibroblasts in vitro. Wound Repair Regen. 2009; 17:240-249. [PubMed: 19320893]

754. Wu X, Shen H, Yu L, Peng M, Lai WS, Ding YL. Corticotropin-releasing hormone activates connexin 43 via activator protein-1 transcription factor in human myometrial smooth muscle cells. Am J Physiol Endocrinol Metab. 2007; 293:E1789-E1794. [PubMed: 17895291]

755. Xing D, Kjolbye AL, Nielsen MS, Petersen JS, Harlow KW, Holstein-Rathlou NH, Martins JB. ZP123 increases gap junctional conductance and prevents reentrant ventricular tachycardia during myocardial ischemia in open chest dogs. J Cardiovasc Electrophysiol. 2003; 14:510-520. [PubMed: 12776869]

756. Xu X, Berthoud VM, Beyer EC, Ebihara L. Functional role of the carboxyl terminal domain of human Connexin 50 in gap junctional channels. J Membr Biol. 2002; 186:101-112. [PubMed: 11944087] 
757. Yamamoto T, Shiosaka S, Whittaker ME, Hertzberg EL, Nagy JI. Gap junction protein in rat hippocampus: Light microscope immunohistochemical localization. J Comp Neurol. 1989; 281:269-281. [PubMed: 2540226]

758. Yamamoto Y, Klemm MF, Edwards FR, Suzuki H. Intercellular electrical communication among smooth muscle and endothelial cells in guinea-pig mesenteric arterioles. J Physiol. 2001; 535:181-195. [PubMed: 11507168]

759. Yamasaki H, Naus CC. Role of connexin genes in growth control. Carcinogenesis. 1996; 17:1199-1213. [PubMed: 8681433]

760. Yang B, Lin H, Xiao J, Lu Y, Luo X, Li B, Zhang Y, Xu C, Bai Y, Wang H, Chen G, Wang Z. The muscle-specific microRNA miR-1 regulates cardiac arrhythmogenic potential by targeting GJA1 and KCNJ2. Nat Med. 2007; 13:486-491. [PubMed: 17401374]

761. Yano T, Ito F, Kobayashi K, Yonezawa Y, Suzuki K, Asano R, Hagiwara K, Nakazawa H, Toma $\mathrm{H}$, Yamasaki H. Hypermethylation of the $\mathrm{CpG}$ island of connexin 32, a candiate tumor suppressor gene in renal cell carcinomas from hemodialysis patients. Cancer Lett. 2004; 208:137-142. [PubMed: 15142671]

762. Ye ZC, Wyeth MS, Baltan-Tekkok S, Ransom BR. Functional hemichannels in astrocytes: A novel mechanism of glutamate release. J Neurosci. 2003; 23:3588-3596. [PubMed: 12736329]

763. Yeager, M. Gap Junction Channel Structure. In: Harris, AL.; Locke, D., editors. Connexins, A Guide. Humana Press; New York: 2009. p. 27-75.

764. Yeh HI, Chou Y, Liu HF, Chang SC, Tsai CH. Connexin37 gene polymorphism and coronary artery disease in Taiwan. Int J Cardiol. 2001; 81:251-255. [PubMed: 11744143]

765. Yi ZC, Wang H, Zhang GY, Xia B. Downregulation of connexin 43 in nasopharyngeal carcinoma cells is related to promoter methylation. Oral Oncol. 2007; 43:898-904. [PubMed: 17306607]

766. Yin X, Gu S, Jiang JX. The Development-associated cleavage of lens Connexin 45.6 by caspase-3-like protease is regulated by casein kinase II-mediated phosphorylation. J Biol Chem. 2001; 276:34567-34572. [PubMed: 11448971]

767. Yogo K, Ogawa T, Akiyama M, Ishida N, Takeya T. Identification and functional analysis of novel phosphorylation sites in Cx43 in rat primary granulosa cells. FEBS Letters. 2002; 531:132-136. [PubMed: 12417300]

768. Yogo K, Ogawa T, Akiyama M, Ishida-Kitagawa N, Sasada H, Sato E, Takeya T. PKA implicated in the phosphorylation of $\mathrm{Cx} 43$ induced by stimulation with FSH in rat granulosa cells. J Rep Dev. 2006; 52:321-328.

769. Yoo B, Lemaire A, Mangmool S, Wolf MJ, Curcio A, Mao L, Rockman HA. +|1-Adrenergic receptors stimulate cardiac contractility and CaMKII activation in vivo and enhance cardiac dysfunction following myocardial infarction. Am J Physiol Heart Circ Physiol. 2009; 297:H1377-H1386. [PubMed: 19633206]

770. You S, Li W, Lin T. Expression and regulation of connexin43 in rat Leydig cells. J Endocrinol. 2000; 166:447-453. [PubMed: 10927634]

771. Young RC, Schumann R, Zhang P. The signaling mechanisms of long distance intercellular calcium waves (far waves) in cultured human uterine myocytes. J Muscle Res Cell Motil. 2002; 23:279-284. [PubMed: 12630701]

772. Yu J, Bippes CA, Hand GM, Muller DJ, Sosinsky GE. Aminosulfonate modulated pH-induced conformational changes in connexin26 hemichannels. J Biol Chem. 2007; 282:8895-8904. [PubMed: 17227765]

773. Yue P, Zhang Y, Du Z, Xiao J, Pan Z, Wang N, Yu H, Ma W, Qin H, Wang WH, Lin DH, Yang B. Ischemia impairs the association between connexin 43 and $\mathrm{M} 3$ subtype of acetylcholine muscarinic receptor (M3-mAChR) in ventricular myocytes. Cell Physiol Biochem. 2006; 17:129-136. [PubMed: 16543729]

774. Zahler S, Hoffmann A, Gloe T, Pohl U. Gap-junctional coupling between neutrophils and endothelial cells: A novel modulator of transendothelial migration. J Leukoc Biol. 2003; 73:118126. [PubMed: 12525569]

775. Zamir O, Hanani M. Intercellular dye-coupling in intestinal smooth muscle. Are gap junctions required for intercellular coupling? Experientia. 1990; 46:1002-1005. [PubMed: 2226711] 
776. Zampighi GA, Loo DD, Kreman M, Eskandari S, Wright EM. Functional and morphological correlates of connexin50 expressed in Xenopus laevis oocytes. J Gen Physiol. 1999; 113:507524. [PubMed: 10102933]

777. Zhang J, Hill CE. Differential connexin expression in preglomerular and postglomerular vasculature: Accentuation during diabetes. Kidney Int. 2005; 68:1171-1185. [PubMed: 16105048]

778. Zhang JT, Chen M, Foote CI, Nicholson BJ. Membrane integration of in vitro-translated gap junctional proteins: Co- and post-translational mechanisms. Mol Biol Cell. 1996; 7:471-482. [PubMed: 8868474]

779. Zhang Y, Tang W, Ahmad S, Sipp JA, Chen P, Lin X. Gap junction-mediated intercellular biochemical coupling in cochlear supporting cells is required for normal cochlear functions. Proc Natl Acad Sci U S A. 2005; 102:15201-15206. [PubMed: 16217030]

780. Zhang YW, Kaneda M, Morita I. The gap junction-independent tumorsuppressing effect of connexin 43. J Biol Chem. 2003; 278:44852-44856. [PubMed: 12952975]

781. Zhao HB, Yu N, Fleming CR. Gap junctional hemichannel-mediated ATP release and hearing controls in the inner ear. Proc Natl Acad Sci U S A. 2005; 102:18724-18729. [PubMed: 16344488]

782. Zheng-Fischhofer Q, Ghanem A, Kim JS, Kibschull M, Schwarz G, Schwab JO, Nagy J, Winterhager E, Tiemann K, Willecke K. Connexin31 cannot functionally replace connexin43 during cardiac morphogenesis in mice. J Cell Sci. 2006; 119:693-701. [PubMed: 16449318]

783. Zhou Y, Yang W, Lurtz MM, Chen Y, Jiang J, Huang Y, Louis CF, Yang JJ. Calmodulin mediates the Ca2+-dependent regulation of $\mathrm{Cx} 44$ gap junctions. Biophys J. 2009; 96:2832-2848. [PubMed: 19348766]

784. Zhou Y, Yang W, Lurtz MM, Ye Y, Huang Y, Lee HW, Chen Y, Louis CF, Yang JJ. Identification of the calmodulin binding domain of connexin 43. J Biol Chem. 2007; 282:3500535017. [PubMed: 17901047]

785. Zhu WZ, Wang SQ, Chakir K, Yang D, Zhang T, Brown JH, Devic E, Kobilka BK, Cheng H, Xiao RP. Linkage of beta-1-adrenergic stimulation to apoptotic heart cell death through protein kinase A-ndependent activation of Ca2+/calmodulin kinase II. J Clin Invest. 2003; 111:617-625. [PubMed: 12618516]

786. Zlomuzica A, Reichinnek S, Maxeiner S, Both M, May E, Worsdorfer P, Draguhn A, Willecke K, Dere E. Deletion of connexin45 in mouse neurons disrupts one-trial object recognition and alters kainate-induced gamma-oscillations in the hippocampus. Physiol Behav. 2010; 101:245-253. [PubMed: 20471991]

787. Zoidl G, Dermietzel R. Gap junctions in inherited human disease. Pflugers Arch. 2010; 460:451466. [PubMed: 20140684] 

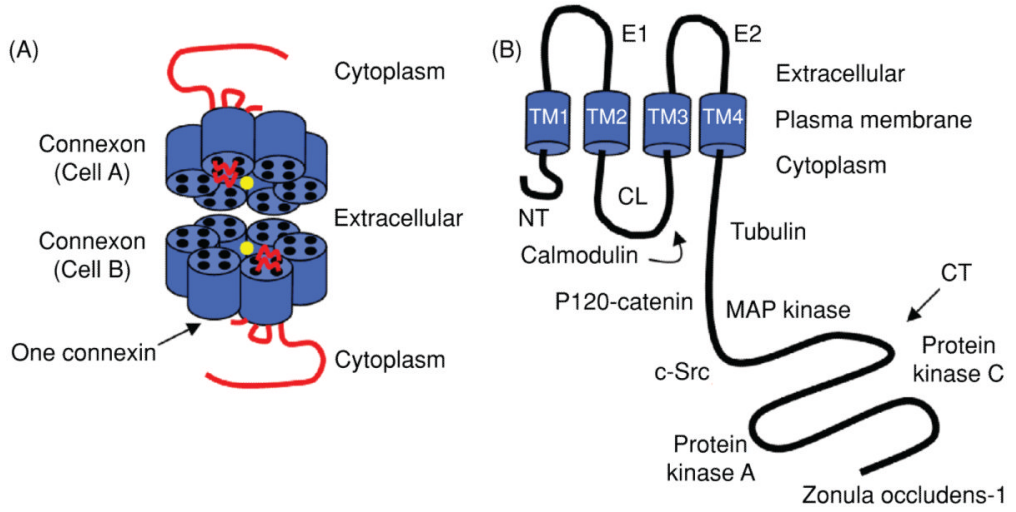

Figure 1.

Model of a Cx43 gap junction channel and monomer. (A) The channel pore location has been indicated by the yellow circle. (B) The $\mathrm{Cx} 43$ monomer with protein partners. The abbreviations are as follows: NT, N-terminus; CL, cytoplasmic loop; CT, C-terminus; E1 and E2, extracellular loops 1 and 2; TM1-4, transmembrane segments 1-4. 

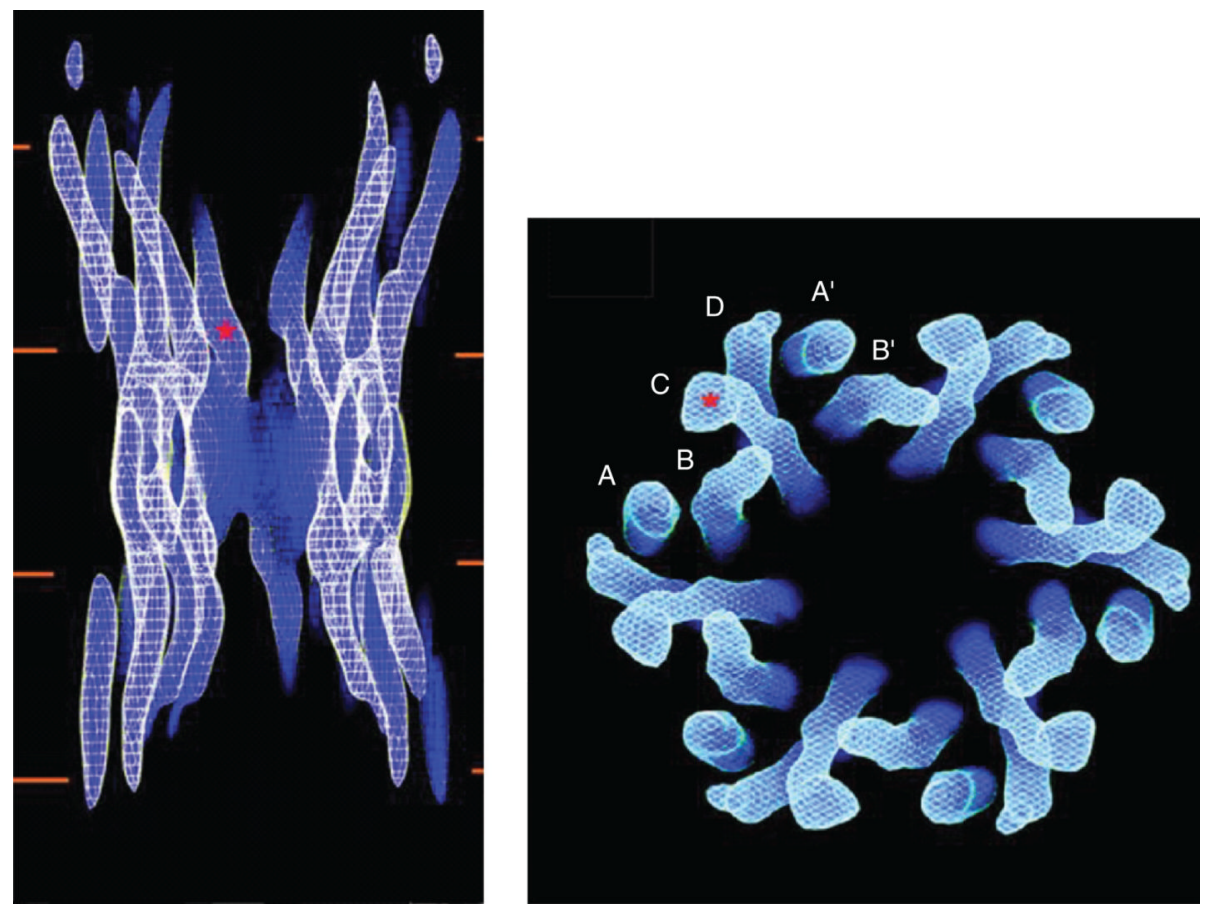

Figure 2.

Cx43 channel structure obtained by electron crystallography. The panel on the left shows a side view of the entire channel. The red lines represent the lipid bilayers. The red asterisk indicates the point at which the pore diameter is estimated to be the smallest. The panel on the right is a view from the cytoplasmic side. The channel is formed by six repeats of four identifiable densities (A-D), each density corresponding to one transmembrane domain. Modified, with permission, from Unger VM, Kumar NM, Gilula NB, Yeager M. Science 283: 1176-1180, 1999 (698). Reprinted, with permission, from AAAS. 


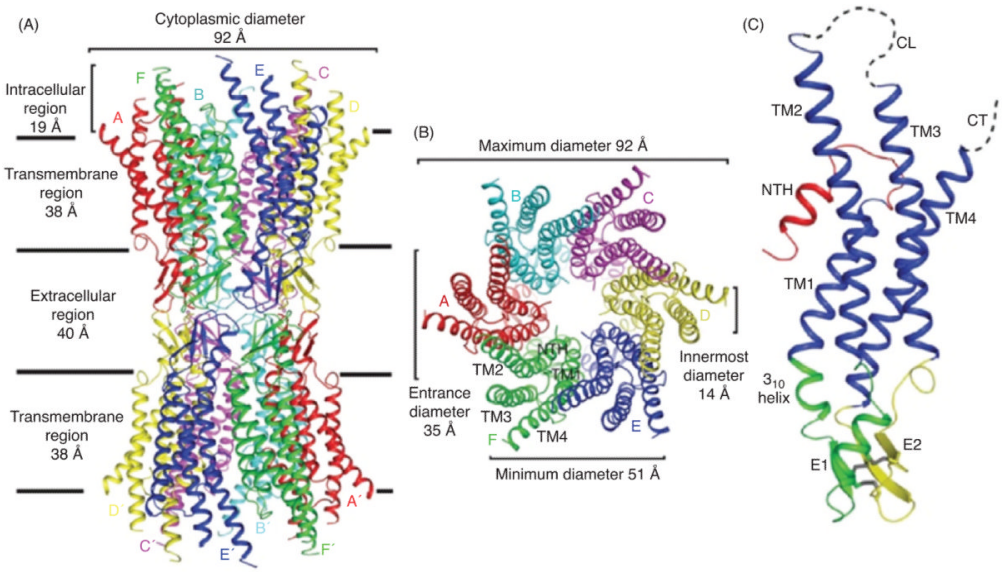

Figure 3.

Structure of the Cx26 gap junction channel and Cx26 protomer in ribbon representation. (A) Side view of the Cx26 gap junction channel. (B) Top view of the Cx26 gap junction channel showing the arrangement of the transmembrane helices TM1 to TM4. (C) Side view of the Cx26 protomer. Color code: red, NT; blue, TM1-TM4; green, E1; yellow, E2; gray, disulphide bonds; dashed lines, CL and CT, which were not visible in the map. E1 and E2 are the loops connecting TM1 and TM2, and TM3 and TM4, respectively. Modified, with permission, from Maeda et al. 2009 (409). Reprinted by permission from Macmillan Publishers Ltd: Nature (458: 597-602), copyright [2009]. 


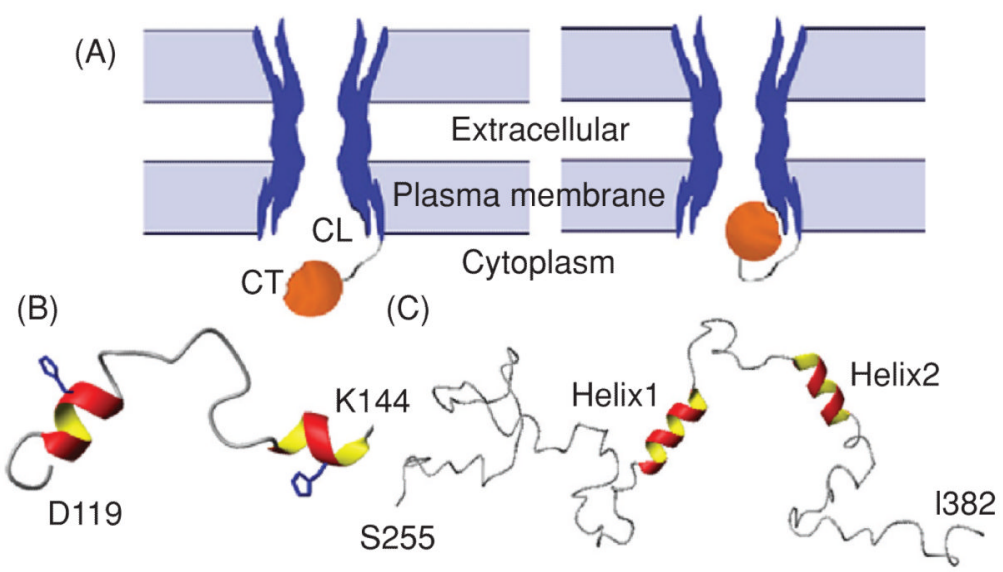

Figure 4.

"Ball-and-chain" model of Cx43 regulation. (A) Under normal conditions, the gate [cytoplasmic tail (CT)] is away from the pore. Under the appropriate stimulus, the gate swings toward the mouth of the channel, binds to a receptor [cytoplasmic loop (CL)] affiliated with the pore, and closes the channel. Lowest energy structure of the (B) Cx43CL and (C) Cx43CT domains; a-helices colored red and yellow. Figure is modified, with permission, from Delmar M, Coombs W, Sorgen P, Duffy HS, Taffet SM, Structural bases for the chemical regulation of Connexin43 channels, Cardiovasc.Res., 2003, 62(2): 268-275, (138) by permission of Oxford University Press, Duffy et al. 2002 (155), and Sorgen et al. 2004 (648), with permission. 
(A)

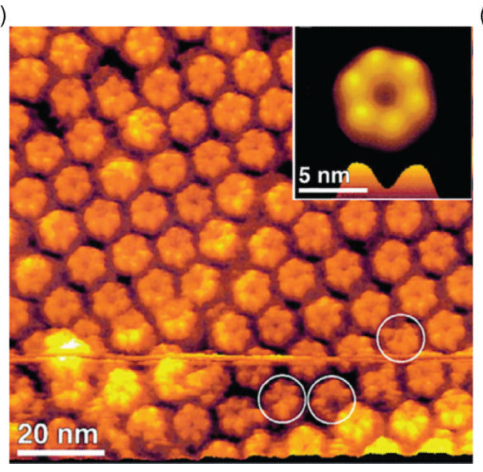

(B)

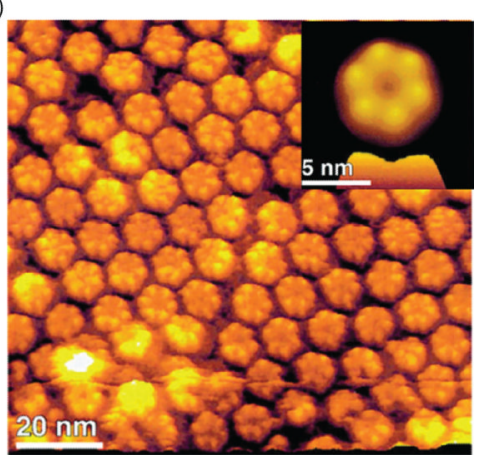

Figure 5.

Conformational changes in Cx26 hemichannels observed in low and high calcium buffers by atomic force microscopy (AFM). (A) AFM topograph showing the extracellular connexon surface imaged in a calcium-free buffer solution. Individual connexons exhibit defects in the number of subunits, as indicated by the circles. (B) Same connexon surface imaged in (A), but in the presence of $0.5 \mathrm{mmol} / \mathrm{L}$ calcium. The channel diameter has changed significantly as seen in the correlation averaged top view (inset) and the profile at the bottom of the inset. All images were displayed as relief tilted by $5^{\circ}$. (Modified, with permission, from Müller et al. 2002 (456). Reprinted by permission from Macmillan Publishers Ltd: EMBO J (21:

3598-3607), copyright [2002]). 


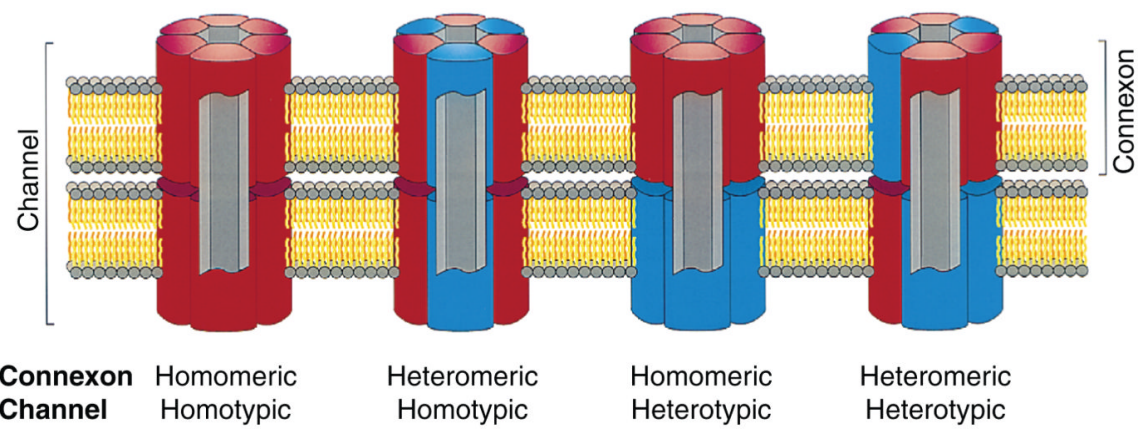

Figure 6.

Possible channels formed by multiple connexins. The figure shows gap junctional channels of different composition. Homomeric connexons are formed by a single connexin type whereas connexons containing more than one connexin type is heteromeric. When connexons of the same composition form a cell-cell channel it is homotypic and if the connexons differ in composition it is heterotypic. Reprinted from Cell, 84(3), Kumar NM, Gilula NB, The gap junction communication channel, 381-8, Copyright [1996], (338) with permission from Elsevier. 


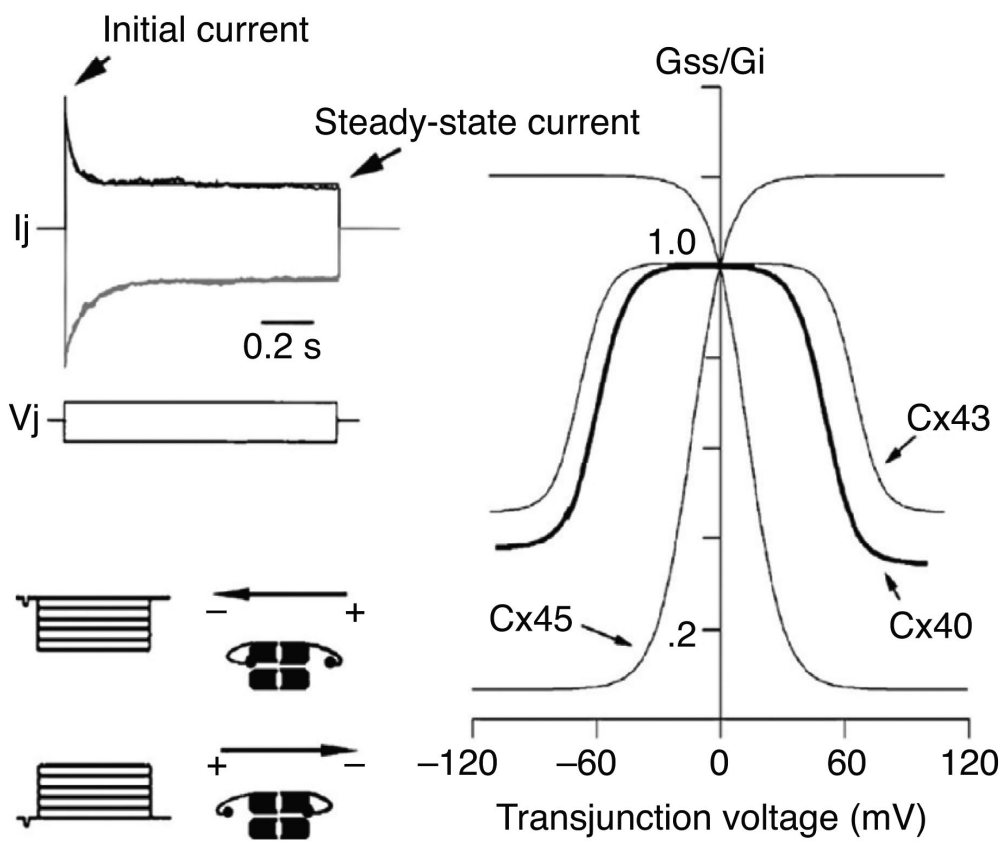

Figure 7.

Voltage dependence of connexin channels. Upper left panel shows the current elicited by imposing a plus or minus $100 \mathrm{mV}$ gradient across $\mathrm{Cx} 43$ channels. The initial current that decays over time until it reaches a lower steady-state current. Right panel shows a plot of the fractional conductance (steady-state conductance (Gss) divided by the initial conductance (Gi)] as a function of transjunctional voltage for $\mathrm{Cx} 40, \mathrm{Cx} 43$, and $\mathrm{Cx} 45$. Lower left panel demonstrates the concept of gating polarity of $\mathrm{Cx} 43$ (negative gating polarity). If the voltage gradient is sufficiently large the gating particle will close the connexion that is relatively negative on the cytoplasmatic side. Figure adapted from Moreno AP, Biophysical properties of homomeric and heteromultimeric channels formed by cardiac connexins,

Cardiovasc.Res., 2002, 62(2):276-86, (448) by permission of Oxford University Press. 
(A)

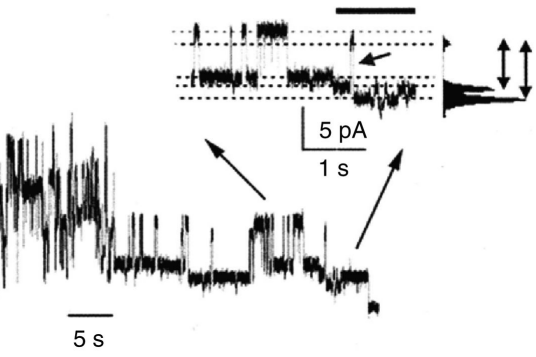

(B)

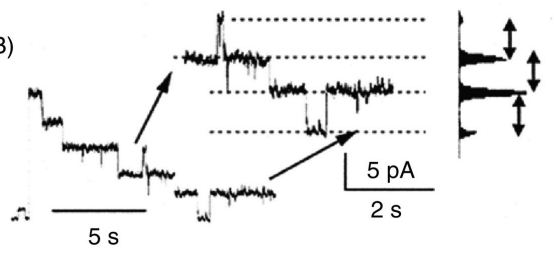

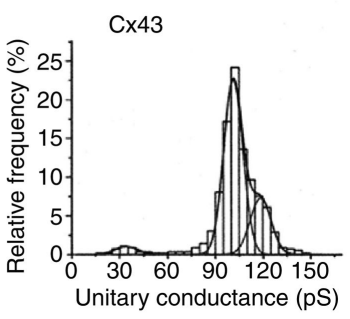

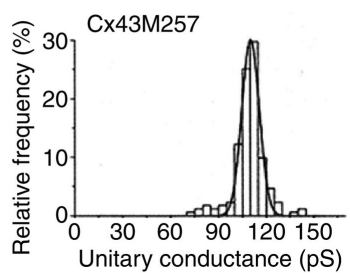

Figure 8.

Effect of cytoplasmic tail (CT) truncation on fast $\mathrm{V}_{\mathrm{j}}$ gating in $\mathrm{Cx} 43$. (A) Left: trace of channel activity recorded at a transjunctional voltage of $-60 \mathrm{mV}$. In the expanded inset, shows clear transitions between the main open and residual state (fast gating). Right: allevents histogram showing the distribution of the observed conductance events. (B) Recording of activity by CT-truncated Cx43 channels at $\mathrm{V}_{\mathrm{j}}=-60 \mathrm{mV}$. The trace and allevents histogram shows that only gating between the main open and closed state was observed. Figure adapted from Moreno AP, Chanson M, Elenes S, Anumonwo J, Scerri I, $\mathrm{Gu}$ H, Taffet SM, Delmar M, Role of the carboxyl terminal of connexin43 in transjunctional fast voltage gating, Circ.Res., 90(4):450-7, 2002 (449), with permission. 


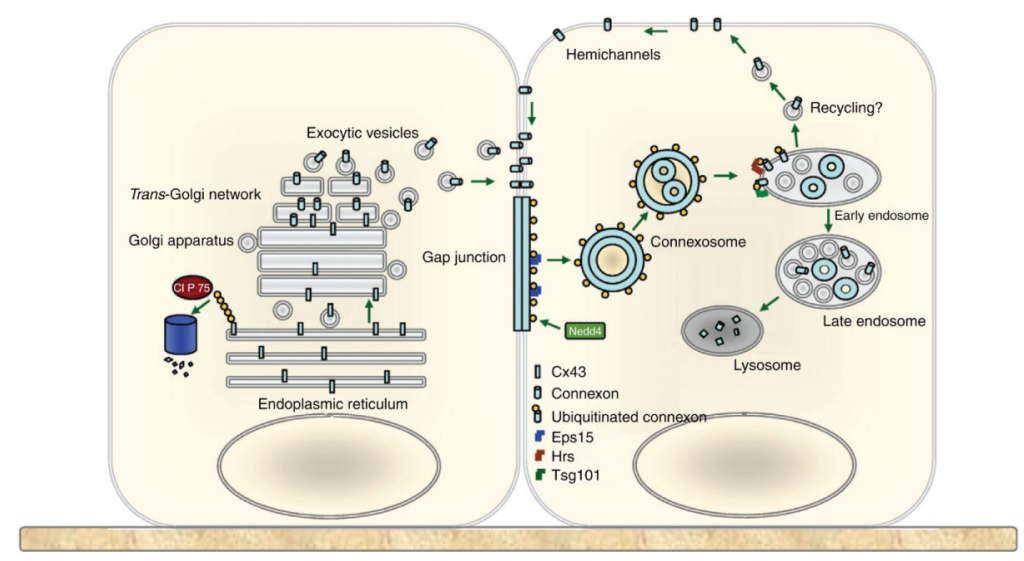

Figure 9.

Role of ubiquitination in trafficking and degradation of $\mathrm{Cx} 43$. The left cell summarizes the events of polyubiquitination followed by ERAD. The right cells summarizes internalization and lysosomal degradation after Cx43 monoubiquitination. Reprinted from Cell Signal., Vol 22, Kjenseth A, Fykerud T, Rivedal E, Leithe E, Regulation of gap junction intercellular communication by the ubiquitin system, 1267-73, Copyright [2010] (317), with permission from Elsevier. 


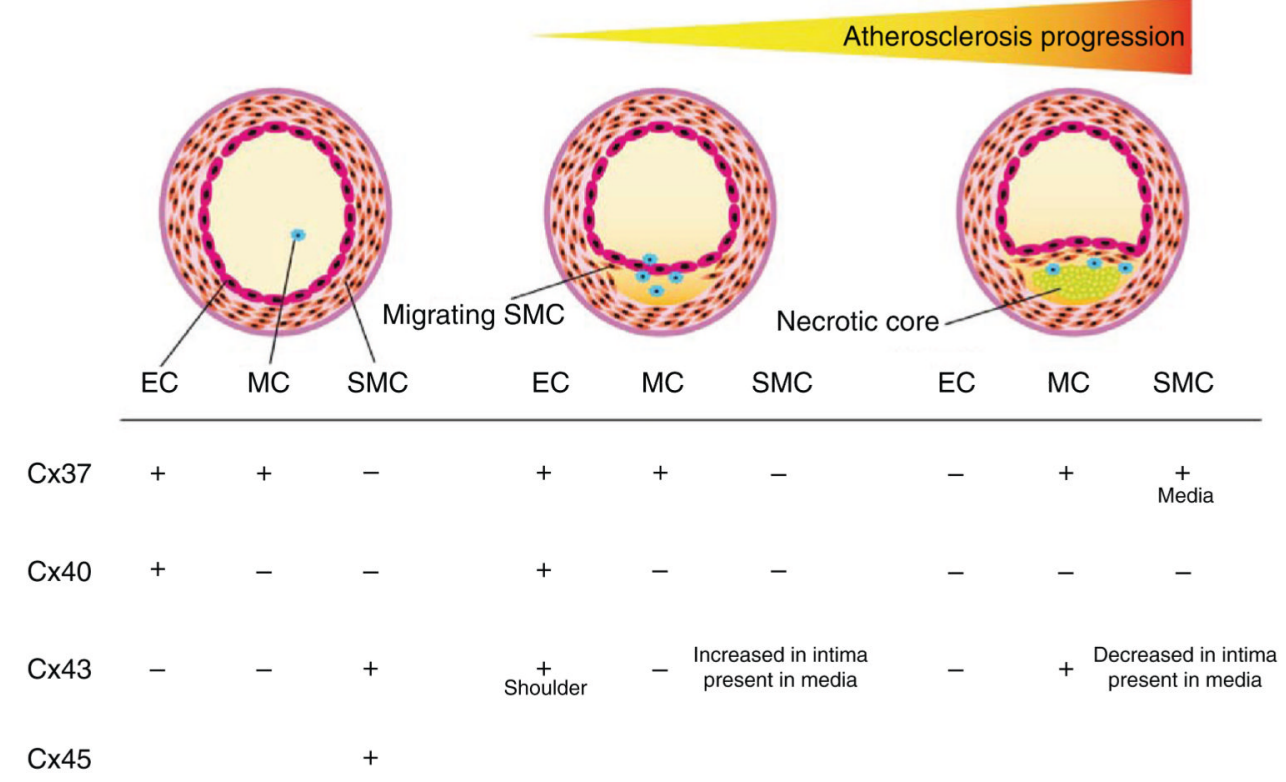

Figure 10.

Expression of connexins in normal vessels and during progression of artherosclerosis. The expression of $\mathrm{Cx} 37, \mathrm{Cx} 40, \mathrm{Cx} 43$, and $\mathrm{Cx} 45$ is indicated for the different cell types involved [endothelial cells (ECs), smooth muscle cells (SMCs), and monocytes (MCs)]. Adapted with kind permission from Springer Science+Business Media: Semin.Immunopathol., Connexins participate in the initiation and progression of atherosclerosis, 31, 2009, 49-61, Morel S, Burnier L, Kwak BR, figure 3, (447). 
(A)

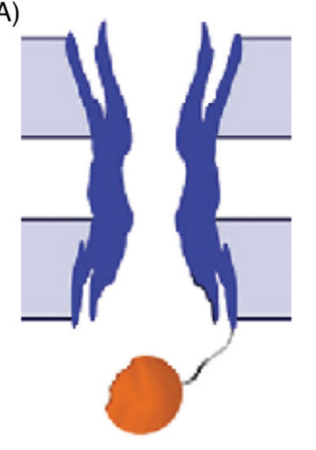

(B)

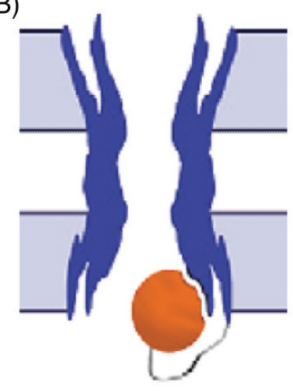

(C)

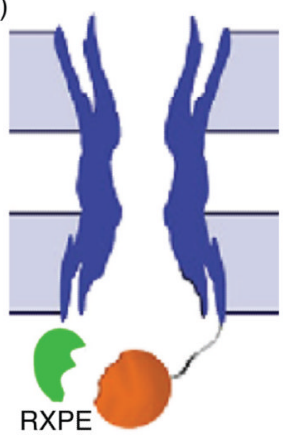

Figure 11.

RXP-E prevents particle-receptor interaction and channel closure. (A) Open channel. (B) Channel closed by particle-receptor interaction. (C) RXP-E binds the cytoplasmic tail (CT) and prevents closure by interrupting binding to the receptor. Modified from Delmar M, Coombs W, Sorgen P, Duffy HS, Taffet SM, Structural bases for the chemical regulation of Connexin43 channels, Cardiovasc.Res., 2004, 62(2): 268-75, (138), by permission of Oxford University Press. 
Table 1

Human and Mouse Connexin Family Members

\begin{tabular}{|c|c|c|}
\hline GJ & $\mathbf{h C x}$ & $\mathbf{m C x}$ \\
\hline $\mathrm{GJ} \epsilon 1$ & $\mathrm{hCx} 23$ & $\mathrm{mCx} 23$ \\
\hline GJ $\beta 7$ & $\mathrm{hCx} 25$ & - \\
\hline GJ $\beta 2$ & $\mathrm{hCx} 26$ & $\mathrm{mCx} 26$ \\
\hline GJץ3 & hCx30.2 & $\mathrm{mCx} 29$ \\
\hline GJ $\beta 6$ & $\mathrm{hCx} 30$ & $\mathrm{mCx} 30$ \\
\hline GJ $\beta 4$ & $\mathrm{hCx} 30.3$ & $\mathrm{mCx} 30.3$ \\
\hline GJ $\beta 3$ & $\mathrm{hCx} 31$ & $\mathrm{mCx} 31$ \\
\hline GJ $\beta 5$ & $\mathrm{hCx} 31.1$ & $\mathrm{mCx} 31.1$ \\
\hline GJ 8 & hCx31.9 & $\mathrm{mCx} 30.2$ \\
\hline GJ $\beta 1$ & $\mathrm{hCx} 32$ & $\mathrm{mCx} 32$ \\
\hline- & - & $\mathrm{mCx} 33$ \\
\hline GJ $\delta 2$ & hCx36 & $\mathrm{mCx} 36$ \\
\hline GJa4 & $\mathrm{hCx} 37$ & $\mathrm{mCx} 37$ \\
\hline GJa5 & $\mathrm{hCx} 40$ & $\mathrm{mCx} 40$ \\
\hline GJ $\delta 4$ & $\mathrm{hCx} 40.1$ & $\mathrm{mCx} 39$ \\
\hline GJa1 & $\mathrm{hCx} 43$ & $\mathrm{mCx} 43$ \\
\hline GJY1 & $\mathrm{hCx} 45$ & $\mathrm{mCx} 45$ \\
\hline GJa3 & $\mathrm{hCx} 46$ & $\mathrm{mCx} 46$ \\
\hline GJY2 & $\mathrm{hCx} 47$ & $\mathrm{mCx} 47$ \\
\hline GJa8 & hCx 50 & $\mathrm{mCx} 50$ \\
\hline GJa9 & hCx59 & - \\
\hline GJa10 & $\mathrm{hCx} 62$ & $\mathrm{mCx} 57$ \\
\hline
\end{tabular}


Table 2

Voltage Dependence of Selected Connexins

\begin{tabular}{llllll}
\hline Connexin & $\begin{array}{l}\mathbf{V}_{\mathbf{1 / 2}} \\
\text { (half-inactivation voltage, } \mathbf{m V})\end{array}$ & $\mathbf{G}_{\mathbf{m a x}}$ & $\mathbf{G}_{\mathbf{m i n}}$ & $\begin{array}{l}\text { Single channel } \\
\text { conductance, } \mathbf{\gamma}, \mathbf{p S}\end{array}$ & References \\
\hline 26 & 95 & $0.94 / 1.01$ & $0.19 / 0.19$ & $115-150$ & $(35,226,326)$ \\
32 & $60 / 63$ & $1.02 / 1 / 01$ & $0.25 / 0.27$ & 70 & $(482,559)$ \\
40 & 35 & $1 / 1$ & $0.19 / 0.19$ & $158-198$ & $(9,33)$ \\
43 & $61 / 61$ & $1 / 1$ & $0.29 / 0.29$ & $90-110$ & $(70,449)$ \\
45 & $23 / 21$ & $1.16 / 1.21$ & $0.08 / 0.07$ & 30 & $(24)$ \\
\hline
\end{tabular}

The voltage dependence can be described by the $\mathrm{V}_{\mathrm{j}}$ at which half maximal inactivation occurs $\left(\mathrm{V}_{1 / 2}\right)$, the fractional maximum conductance relative to the conductance at $\mathrm{V}_{\mathrm{j}}=0\left(\mathrm{G}_{\max }\right)$, and the fractional minimum conductance during inactivation by a large $\mathrm{V}_{\mathrm{j}}\left(\mathrm{G}_{\mathrm{min}}\right)$. 


\section{Table 3}

Cx43 Phosphorylation Sites

\begin{tabular}{lll}
\hline Residue & Kinase(s) & Reference(s) \\
\hline S244 & CaMKII & $(282)$ \\
Y247 & v-Src & $(383,645)$ \\
S255 & MAPK/CaMKII & $(80,282,739)$ \\
S257* & PKG/CaMKII & $(282,342,344)$ \\
S262 & PKC $\epsilon$ & $(14,148,657)$ \\
Y265 & v-Src & $(383,645,687)$ \\
S279 & MAPK & $(739)$ \\
S282 & MAPK & $(739)$ \\
S296 & CaMKII & $(14,282)$ \\
S297 & CaMKII & $(14,282)$ \\
S306 & CaMKII & $(14,282)$ \\
S314 & CaMKII & $(282)$ \\
S325 & CK1/CaMKII & $(109,282)$ \\
S328 & CK1/CaMKII & $(109,282)$ \\
S330 & CK1/CaMKII & $(109,282)$ \\
S364 & PKA/CaMKII & $(282,616,673)$ \\
S365 & PKC/PKA/CaMKII & $(282,616)$ \\
S368 & PKC & $(22,356,579,616)$ \\
S369 & PKC/CaMKII & $(282,616)$ \\
S372 & PKC/CaMKII & $(282,579,616)$ \\
S373 & PKC/CaMKII & $(282,616)$ \\
\hline & &
\end{tabular}

Amino acid residue 257 of rat $\mathrm{Cx} 43$ is a serine. In the human $\mathrm{Cx} 43$ sequence, serine 257 is replaced by alanine. 